Supporting Information for

\title{
Visible Light Irradiation of Acyl Oxime Esters and Styrenes \\ Efficiently Constructs $\beta$-Carbonyl Imides by a Scission and Four- Component Reassembly Process
}

Yuan-Yuan Cheng, Tao Lei, Longlong Su, Xiuwei Fan, Bin Chen, Chen-Ho Tung, and $\mathrm{Li}-\mathrm{Zhu} \mathrm{Wu}^{*}$

*Email of corresponding author: $\underline{\text { lzwu@mail.ipc.ac.cn }}$

\section{Table of Contents}

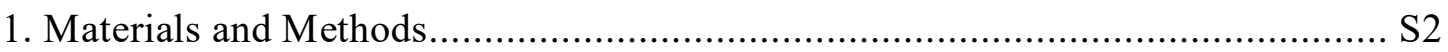

2. General Procedure for Preparation of Oxime Esters …................................. S3

3. Condition Optimization for Reaction of Oxime Ester and Styrene ..................... S4

4. General Procedure for Reaction of Oxime Ester and Styrene.......................... S5

5. Scale-up Reaction of Oxime Ester and Styrene ........................................ S6

6. Optical Spectroscopic and Electrochemical Data .......................................... S7

7. Control Experiments for Mechanistic Study .............................................. S9

8. Characterization Data of All Products...........................................................S13

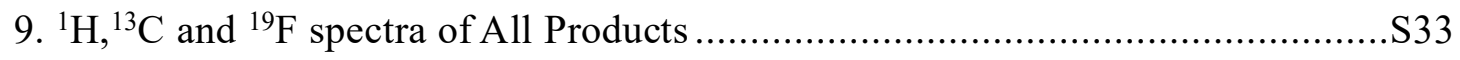

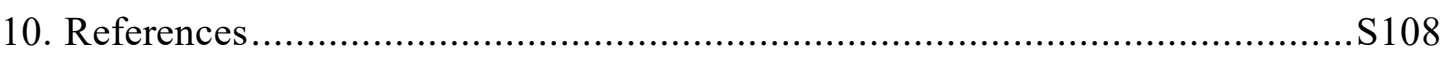




\section{Materials and Methods}

${ }^{1} \mathrm{H}$ NMR, ${ }^{13} \mathrm{C}$ NMR and ${ }^{19} \mathrm{~F}$ NMR (400 MHz, $101 \mathrm{MHz}$ and $377 \mathrm{MHz}$, respectively) spectra were measured in $\mathrm{CDCl}_{3}$ or $\mathrm{CD}_{3} \mathrm{CN}$ recorded on Bruker Avance DPX $400 \mathrm{MHz}$ spectrometer. All chemical shifts $(\delta)$ were reported in ppm and coupling constants $(J)$ in Hz. All chemical shifts were reported relative to tetramethylsilane in $\mathrm{CDCl}_{3}\left(0 \mathrm{ppm}\right.$ for $\left.{ }^{1} \mathrm{H}\right)$ or $\mathrm{CD}_{3} \mathrm{CN}\left(1.94 \mathrm{ppm}\right.$ for $\left.{ }^{1} \mathrm{H}\right)$, and $\mathrm{CDCl}_{3}\left(77.16 \mathrm{ppm}\right.$ for $\left.{ }^{13} \mathrm{C}\right)$, respectively. HRMS (ESI) spectra were recorded on Thermo Scientific Q Exactive Mass Spectrometer. All reagents were purchased from commercial suppliers and used without further purification. Flash chromatography was carried out with silica gel (200-300 mesh). Analytical TLC was performed with silica gel GF254 plates, and the products were visualized by UV detection. Blue LEDs ( $3 \mathrm{~W}, \lambda=460 \mathrm{~nm}, 145 \mathrm{~lm} @ 700 \mathrm{~mA}$ ) were used as the irradiation light. 


\section{General Procedure for Preparation of Oxime Esters}

$\underline{\operatorname{Method~A}}:{ }^{1,2}$

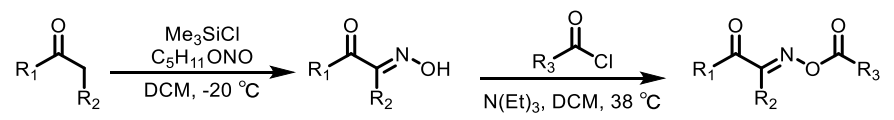

Step 1: To a solution of $10 \mathrm{mmol}$ ketone in $5 \mathrm{~mL}$ DCM was added 1 equiv. TMSCl (1.24 $\mathrm{mL})$ at $20{ }^{\circ} \mathrm{C}$. To this cooled solution was dropwise added 1 equiv. isoamyl nitrite $(1.34 \mathrm{~mL})$. The reaction was found to be instantaneous, but the mixture was stirred at r.t. for an additional period of $1 \mathrm{~h}$ before working up. The solution was directly concentrated in vacuo. The crude product was purified by flash column chromatography and the corresponding oximes was obtained. Step 2: Oxime (1 equiv, $6.1 \mathrm{mmol})$ and triethylamine (1.4 equiv, $8.6 \mathrm{mmol})$ were dissolved in DCE $(20 \mathrm{~mL})$. acyl chloride ( 1.2 equiv, $7.35 \mathrm{mmol}$ ) was gradually added at $0^{\circ} \mathrm{C}$ for 15 minutes. Then, the mixture was stirred at $38{ }^{\circ} \mathrm{C}$ for $12 \mathrm{~h}$ (monitored by TLC). After this time, the mixture was quenched by adding $50 \mathrm{~mL}$ of saturated sodium bicarbonate aqueous solution. The organic layer was separated and washed with $50 \mathrm{~mL}$ of water. After evaporation of the solvent under vacuum, the crude mixture was purified by flash column chromatography (petroleum ether/ethyl acetate).

\section{Method B: ${ }^{3-5}$}

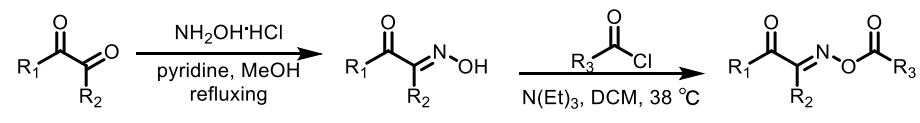

To a solution of $10 \mathrm{mmol}$ 1,2-diketone in $30 \mathrm{~mL} \mathrm{MeOH}$ was added 1.2 equiv. hydroxylamine hydrochloride $(0.863 \mathrm{~g})$ and 1.2 equiv. pyridine $(1.2 \mathrm{~mL})$. The reaction mixture was refluxed for 5 h. Crude oxime was obtained after removal of solvent in vacuo. Crude oxime and triethylamine (1.4 equiv, $14 \mathrm{mmol}$ ) were dissolved in DCE (20 mL). Acyl chloride (1.2 equiv, $12 \mathrm{mmol})$ was gradually added at $0^{\circ} \mathrm{C}$ for 15 minutes. Then, the mixture was stirred at $38{ }^{\circ} \mathrm{C}$ for $12 \mathrm{~h}$ (monitored by TLC). After this time, the mixture was quenched by adding $50 \mathrm{~mL}$ of saturated sodium bicarbonate aqueous solution. The organic layer was separated and washed with $50 \mathrm{~mL}$ of water. After evaporation of the solvent under vacuum, the crude mixture was purified by flash column chromatography (petroleum ether/ethyl acetate). 


\section{Condition Optimization for Reaction of Oxime Ester and Styrene}

Table S1. Optimization of conditions ${ }^{a}$

\begin{tabular}{|c|c|c|c|c|}
\hline Entry & Photocatalyst & Solvent & Time $(h)$ & Yield $^{b}(\%)$ \\
\hline 1 & $1 \mathrm{~mol} \% f a c-\operatorname{Ir}(\mathrm{ppy})_{3}$ & $\mathrm{CH}_{3} \mathrm{CN}$ & 12 & 65 \\
\hline 2 & $1 \mathrm{~mol} \%\left[\operatorname{Ir}(\mathrm{dtbbpy})(\mathrm{ppy})_{2}\right] \mathrm{PF}_{6}$ & $\mathrm{CH}_{3} \mathrm{CN}$ & 12 & 20 \\
\hline 3 & $1 \mathrm{~mol} \% \operatorname{Ir}\left[\mathrm{dF}\left(\mathrm{CF}_{3}\right) \mathrm{ppy}_{2}(\mathrm{dtbpy})\right] \mathrm{PF}_{6}$ & $\mathrm{CH}_{3} \mathrm{CN}$ & 12 & 0 \\
\hline 4 & $1 \mathrm{~mol} \% \mathrm{Ru}(\mathrm{bpy})_{3}(\mathrm{BF} 4)_{2}$ & $\mathrm{CH}_{3} \mathrm{CN}$ & 12 & 0 \\
\hline 5 & $1 \mathrm{~mol} \%$ Eosin $\mathrm{Y}$ & $\mathrm{CH}_{3} \mathrm{CN}$ & 12 & 0 \\
\hline 6 & $1 \mathrm{~mol} \% f a c-\operatorname{Ir}(\mathrm{ppy})_{3}$ & $\mathrm{EtOH}$ & 12 & 0 \\
\hline 7 & $1 \mathrm{~mol} \% f a c-\operatorname{Ir}(\mathrm{ppy})_{3}$ & DMF & 12 & 0 \\
\hline 8 & $1 \mathrm{~mol} \% f a c-\operatorname{Ir}(\mathrm{ppy})_{3}$ & 1,4-Dioxane & 12 & 0 \\
\hline 9 & $1 \mathrm{~mol} \% f a c-\operatorname{Ir}(\mathrm{ppy})_{3}$ & DCE & 12 & $<5$ \\
\hline $10^{c}$ & $1 \mathrm{~mol} \% f a c-\operatorname{Ir}(\mathrm{ppy})_{3}$ & $\mathrm{CH}_{3} \mathrm{CN} / \mathrm{DMF}$ & 12 & 0 \\
\hline $11^{d}$ & $1 \mathrm{~mol} \% f a c-\operatorname{Ir}(\mathrm{ppy})_{3}$ & $\mathrm{CH}_{3} \mathrm{CN}$ & 12 & 52 \\
\hline $12^{e}$ & $1 \mathrm{~mol} \% f a c-\operatorname{Ir}(\mathrm{ppy})_{3}$ & $\mathrm{CH}_{3} \mathrm{CN}$ & 12 & 64 \\
\hline 13 & $1 \mathrm{~mol} \% f a c-\operatorname{Ir}(\mathrm{ppy})_{3}$ & $\mathrm{CH}_{3} \mathrm{CN}$ & 24 & 70 \\
\hline 14 & $1 \mathrm{~mol} \% f a c-\operatorname{Ir}(\mathrm{ppy})_{3}$ & $\mathrm{CH}_{3} \mathrm{CN}$ & 24 & 81 \\
\hline $15^{f}$ & $2 \mathrm{~mol} \% f a c-\operatorname{Ir}(\mathrm{ppy})_{3}$ & $\mathrm{CH}_{3} \mathrm{CN}$ & 24 & 70 \\
\hline 16 & $3 \mathrm{~mol} \% f a c-\operatorname{Ir}(\mathrm{ppy})_{3}$ & $\mathrm{CH}_{3} \mathrm{CN}$ & 24 & 86 \\
\hline $17^{g}$ & $3 \mathrm{~mol} \% f a c-\operatorname{Ir}(\mathrm{ppy})_{3}$ & $\mathrm{CH}_{3} \mathrm{CN}$ & 24 & 54 \\
\hline $18^{h}$ & $3 \mathrm{~mol} \% f a c-\operatorname{Ir}(\mathrm{ppy})_{3}$ & $\mathrm{CH}_{3} \mathrm{CN}$ & 24 & $<5$ \\
\hline $19^{i}$ & $3 \mathrm{~mol} \% f a c-\operatorname{Ir}(\mathrm{ppy})_{3}$ & $\mathrm{CH}_{3} \mathrm{CN}$ & 24 & 71 \\
\hline $20^{j}$ & $3 \mathrm{~mol} \% f a c-\operatorname{Ir}(\mathrm{ppy})_{3}$ & $\mathrm{CH}_{3} \mathrm{CN}$ & 24 & 0 \\
\hline 21 & -- & $\mathrm{CH}_{3} \mathrm{CN}$ & 24 & 0 \\
\hline $22^{k}$ & $3 \mathrm{~mol} \% f a c-\operatorname{Ir}(\mathrm{ppy})_{3}$ & $\mathrm{CH}_{3} \mathrm{CN}$ & 24 & 57 \\
\hline
\end{tabular}

${ }^{a}$ Reaction Condition: oxime ester 1a $(0.2 \mathrm{mmol})$, styrene 2a (5 equiv, $\left.1.0 \mathrm{mmol}\right)$, photocatalyst ( $\left.1 \mathrm{mmol} \%\right)$, $\mathrm{CH}_{3} \mathrm{CN}(2.0 \mathrm{~mL}), \mathrm{Ar}, \mathrm{rt}$, under $460 \mathrm{~nm}$ LEDs irradiation. ${ }^{b}$ Yields detected by ${ }^{1} \mathrm{H}$ NMR. ${ }^{c}$ Volume ratio of $\mathrm{CH}_{3} \mathrm{CN} / \mathrm{DMF}$ was $1: 1 .{ }^{d} 0.5 \mathrm{~mL}$ of $\mathrm{CH}_{3} \mathrm{CN} .{ }^{e} 1.0 \mathrm{~mL}$ of $\mathrm{CH}_{3} \mathrm{CN} .{ }^{f} \mathrm{At} 50{ }^{\circ} \mathrm{C} .{ }^{g} 20 \mathrm{~mol} \%$ tetrabutylammonium hexafluorophosphate was added. ${ }^{h} 20 \mathrm{~mol} \%$ tetrabutylammonium iodide was added. ${ }^{i} 3$ eq styrene was used. ${ }^{j}$ In the absence of light. ${ }^{k}$ In air. 


\section{General Procedure for Reaction of Oxime Ester and Styrene}

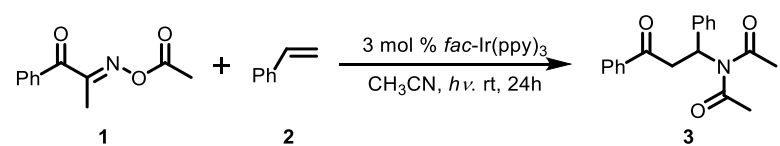

A $10 \mathrm{~mL}$ Pyrex tube equipped with a magnetic stir bar was charged with $1(0.2 \mathrm{mmol})$ and $f a c$ $\operatorname{Ir}(\mathrm{ppy}) 3\left(3.9 \mathrm{mg}, 6 \times 10^{-3} \mathrm{mmol}\right)$ in $\mathrm{CH}_{3} \mathrm{CN}(2.0 \mathrm{~mL})$. This system was bubbled with $\mathrm{Ar}$ for 15 minutes. Then, styrene $2(115 \mathrm{ul}, 1.0 \mathrm{mmol})$ were added into the tube. The tube was sealed and irradiated at room temperature by blue LEDs $(460 \mathrm{~nm})$ for 24 hours. When reaction was finished, the mixture was evaporated to remove the solvent and the residue was purified by flash chromatography on silica gel (eluting with petroleum ether/ethyl acetate $=16: 1$ ) to afford the desired product. 


\section{Scale-up Reaction of Oxime Ester and Styrene}

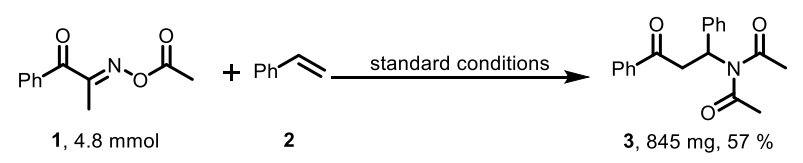

A $200 \mathrm{~mL}$ Pyrex tube equipped with a magnetic stir bar was charged with $1(4.8 \mathrm{mmol})$ and $\mathrm{fac}$ $\operatorname{Ir}\left(\right.$ ppy) $3\left(94.3 \mathrm{mg}, 14.4 \times 10^{-2} \mathrm{mmol}\right)$ in $\mathrm{CH}_{3} \mathrm{CN}(48.0 \mathrm{~mL})$. This system was bubbled with $\mathrm{Ar}$ for 15 minutes. Then, styrene $2(2.76 \mathrm{ml}, 24 \mathrm{mmol})$ were added into the tube. The tube was sealed and irradiated at room temperature by blue LEDs $(460 \mathrm{~nm})$ for 24 hours. When reaction was finished, the mixture was evaporated to remove the solvent and the residue was purified by flash chromatography on silica gel (eluting with petroleum ether/ethyl acetate $=16: 1$ ) to afford the desired product with $57 \%$ yield. 


\section{Optical Spectroscopic and Electrochemical Data}

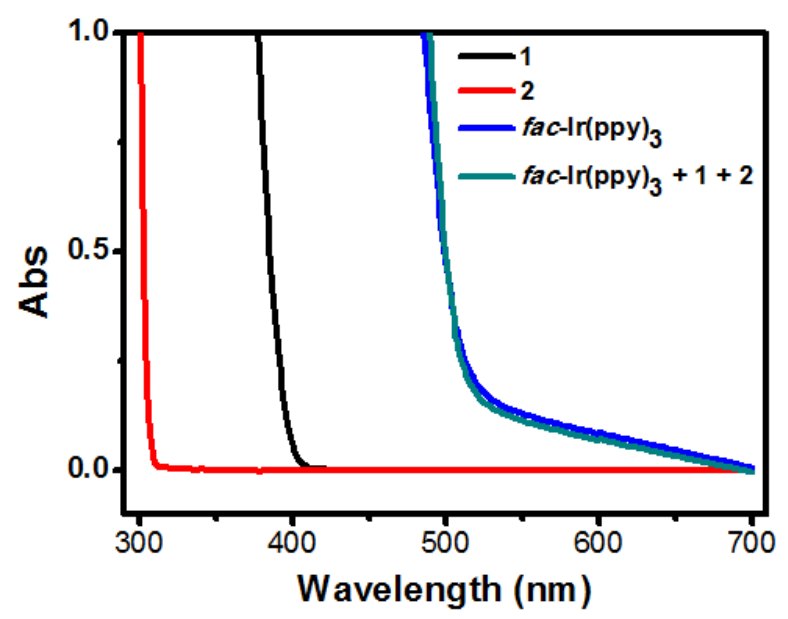

Figure S1. UV-vis absorption spectra of reaction system at reaction concentration: [1] $=0.1 \mathrm{M},[2]$ $=0.5 \mathrm{M},\left[\right.$ fac $\left.-\operatorname{Ir}(\mathrm{ppy})_{3}\right]=3 \times 10^{-3} \mathrm{M}$.

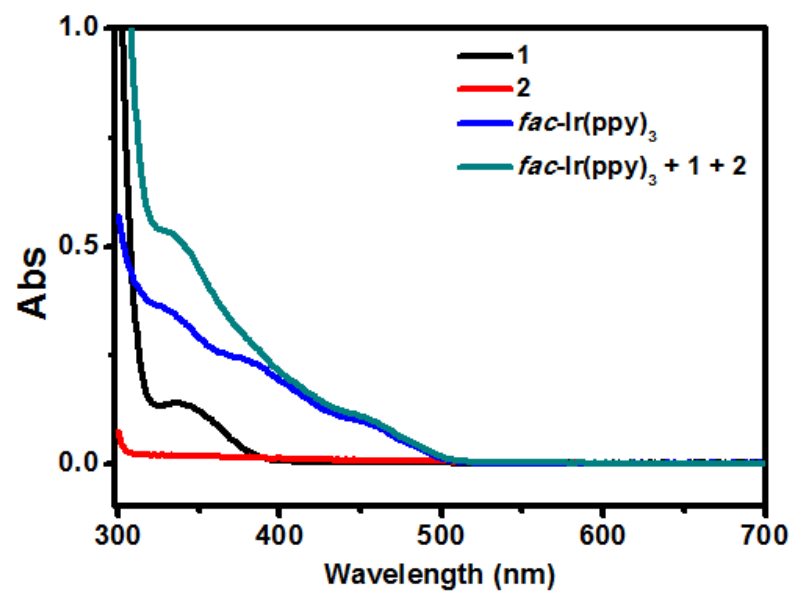

Figure S2. UV-vis absorption spectra of reaction system at low concentration: $[\mathbf{1}]=3.33 \times 10^{-3} \mathrm{M}$, $[2]=1.67 \times 10^{-2} \mathrm{M},\left[\right.$ fac- $\left.\operatorname{Ir}(\mathrm{ppy})_{3}\right]=1 \times 10^{-4} \mathrm{M}$.

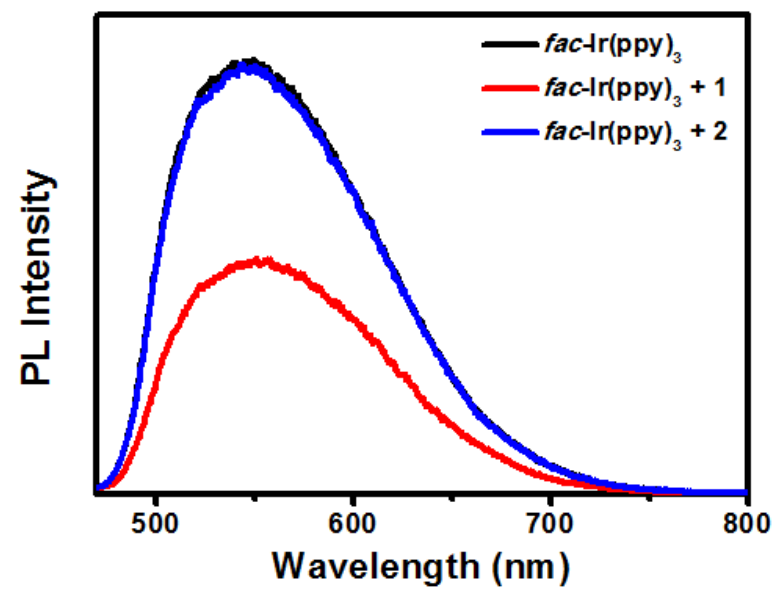

Figure S3. Steady state luminescence quenching experiment in $\mathrm{CH}_{3} \mathrm{CN}$ : $\left[\mathrm{fac}-\operatorname{Ir}(\mathrm{ppy})_{3}\right]=1.00 \times 10^{-5}$ $\mathrm{M},[\mathbf{1}]=3.33 \times 10^{-3} \mathrm{M},[2]=1.67 \times 10^{-2} \mathrm{M}$. Excitation wavelength was $450 \mathrm{~nm}$. 


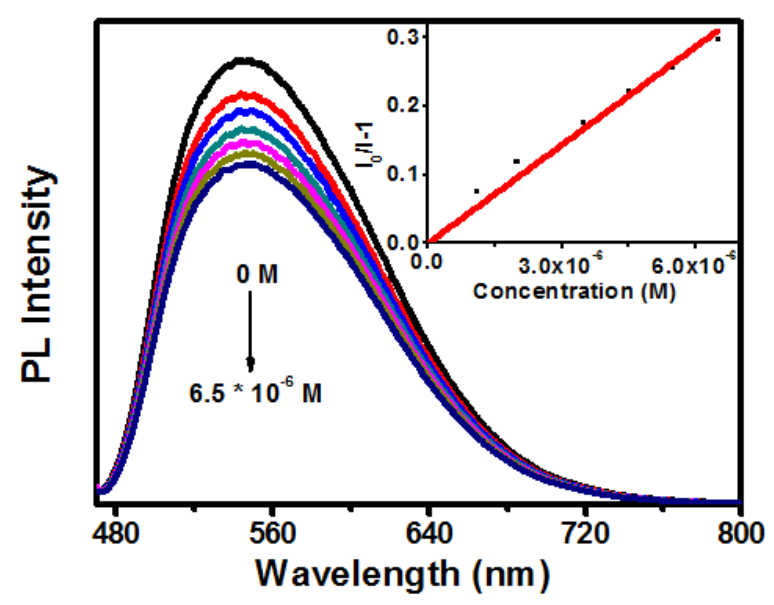

Figure S4. Luminescence spectrum of $f a c-\operatorname{Ir}(\mathrm{ppy})_{3}$ as a function of concentration of oxime ester 1 in degassed $\mathrm{CH}_{3} \mathrm{CN}$ with excitation at $450 \mathrm{~nm},\left[\mathrm{fac}-\operatorname{Ir}(\mathrm{ppy})_{3}\right]=5.0 \times 10^{-5} \mathrm{M}$. Quenching constant ket $=2.52 \times 10^{10} \mathrm{~s}^{-1} \mathrm{M}^{-1}$.

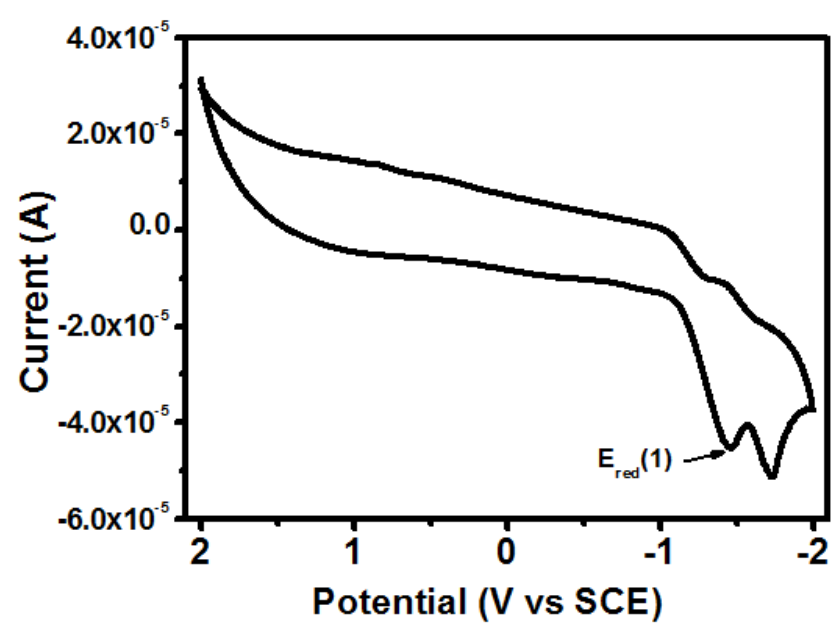

Figure S5. CV experiment of 1 ((E)-2-(acetoxyimino)-1-phenylpropan-1-one) and $\mathrm{NBu}_{4} \mathrm{PF}_{6}$ in degassed $\mathrm{CH}_{3} \mathrm{CN},[\mathbf{1}]=1.00 \times 10^{-3} \mathrm{M},\left[\mathrm{NBu}_{4} \mathrm{PF}_{6}\right]=1.00 \times 10^{-1} \mathrm{M} . \mathrm{E}^{\mathrm{red}}(\mathbf{1})=-1.42 \mathrm{~V}$ SCE .

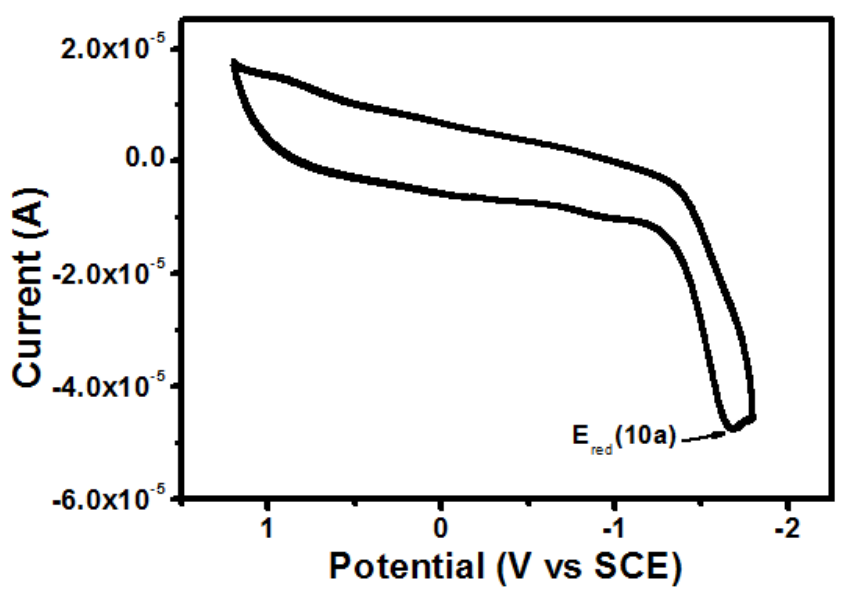

Figure S6. CV experiment of 10a ((E)-3-(acetoxyimino) butan-2-one) and $\mathrm{NBu}_{4} \mathrm{PF}_{6}$ in degassed $\mathrm{CH}_{3} \mathrm{CN},[\mathbf{1}]=1.00 \times 10^{-3} \mathrm{M},\left[\mathrm{NBu}_{4} \mathrm{PF}_{6}\right]=1.00 \times 10^{-1} \mathrm{M} . \mathrm{E}^{\mathrm{red}}(\mathbf{1 0 a})=-1.67 \mathrm{~V} \mathrm{SCE}$. 


\section{Control Experiments for Mechanistic Study}

\section{a) Capture of reaction intermediates:}

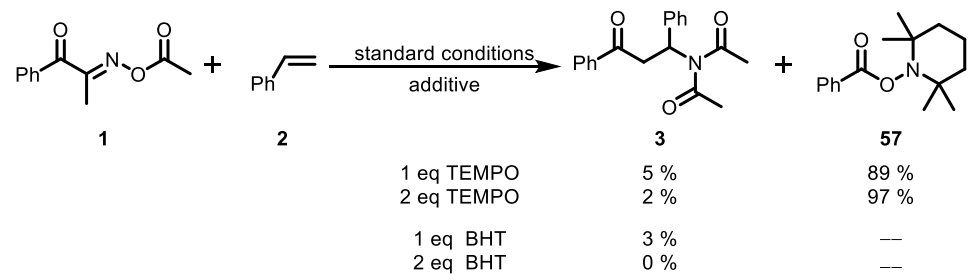

A $10 \mathrm{~mL}$ Pyrex tube equipped with a magnetic stir bar was charged with 1 (0.2 mmol), TEMPO (or BHT) and $f a c-\operatorname{Ir}(\text { ppy })_{3}\left(3.9 \mathrm{mg}, 6 \times 10^{-3} \mathrm{mmol}\right)$ in $\mathrm{CH}_{3} \mathrm{CN}(2.0 \mathrm{~mL})$. This system was bubbled with Ar for 15 minutes. Then, styrene 2 (115 ul, $1.0 \mathrm{mmol}$ ) were added into the tube. The tube was sealed and irradiated at room temperature by blue LEDs (460 nm) for 24 hours. When reaction was finished, the mixture was evaporated to remove the solvent and tested by ${ }^{1} \mathrm{H}$ NMR with 2,2diphenylacetonitrile as internal standard. For 1 equiv. TEMPO, $5 \% \mathbf{3}$ and $89 \% \mathbf{5 7}$ was detected. For 2 equiv. TEMPO, $2 \% 3$ and $97 \% 57$ was detected. For 1 equiv. BHT, $3 \% \mathbf{3}$ was detected. For 2 equiv. BHT, $0 \% 3$ was detected.

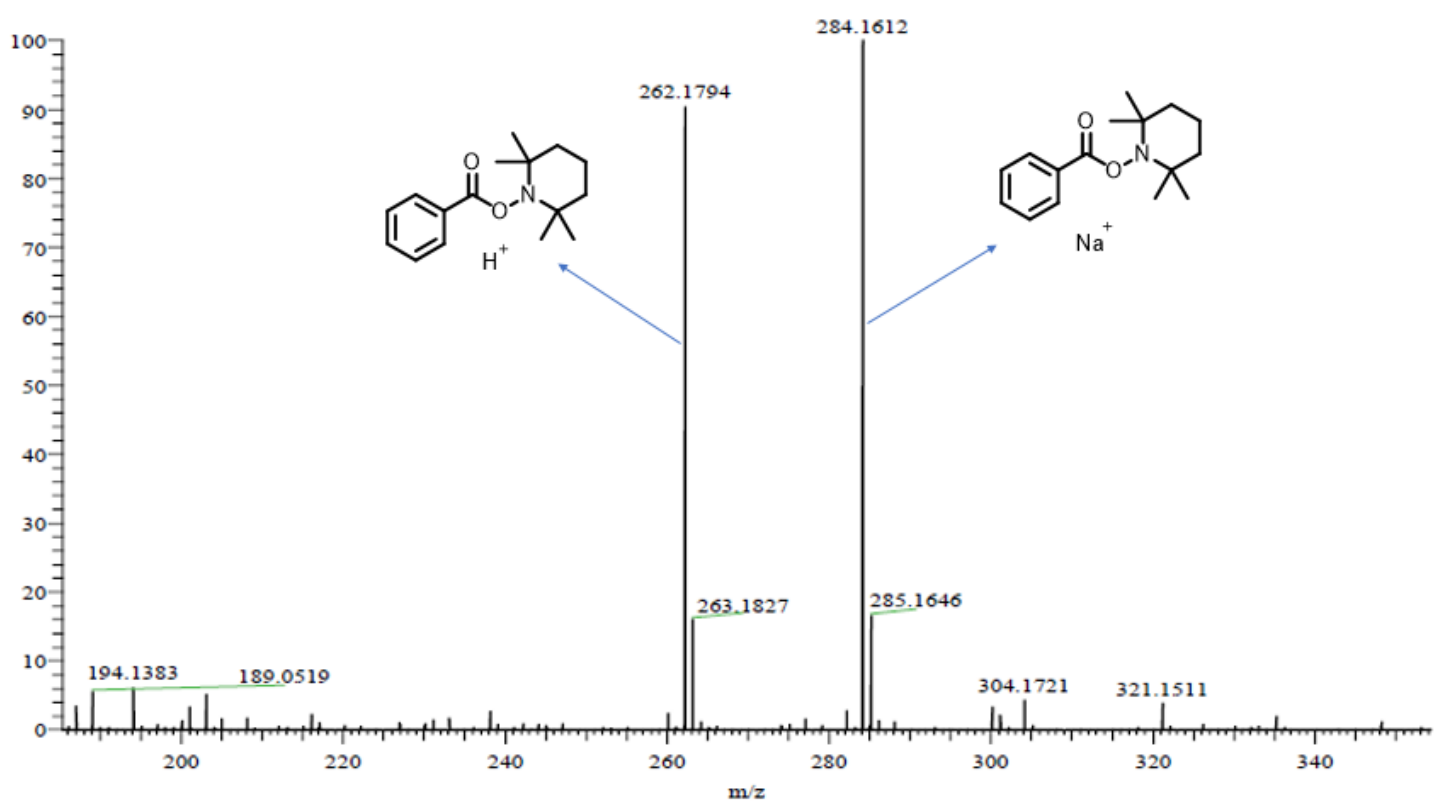

Figure S7. The GC-Mass of capture experiments of reaction intermediates.

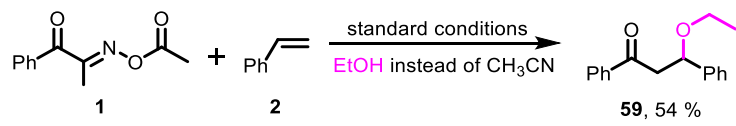


A $10 \mathrm{~mL}$ Pyrex tube equipped with a magnetic stir bar was charged with $1(0.2 \mathrm{mmol})$ and $\mathrm{fac}$ $\operatorname{Ir}(\mathrm{ppy}) 3\left(3.9 \mathrm{mg}, 6 \times 10^{-3} \mathrm{mmol}\right)$ in $\mathrm{CH}_{3} \mathrm{CH}_{2} \mathrm{OH}(2.0 \mathrm{~mL})$. This system was bubbled with Ar for 15 minutes. Then, styrene $2(115 \mathrm{ul}, 1.0 \mathrm{mmol})$ were added into the tube. The tube was sealed and irradiated at room temperature by blue LEDs $(460 \mathrm{~nm})$ for 24 hours. When reaction was finished, the mixture was evaporated to remove the solvent and the residue was purified by flash chromatography on silica gel (petroleum ether/ethyl acetate) to afford the desired product $\mathbf{5 9}$ with $54 \%$ yield of isolated products based on $0.2 \mathrm{mmol} \mathbf{1}$.

\section{b) Deuterium labeling experiment and NMR experiment in situ:}

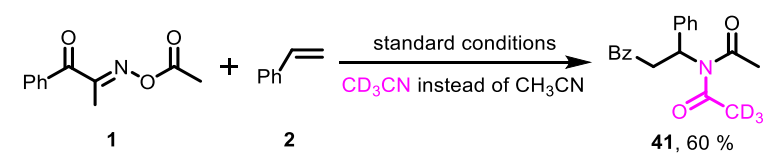

Two $10 \mathrm{~mL}$ Pyrex tubes equipped with a magnetic stir bar were charged with $\mathbf{1}(0.2 \mathrm{mmol})$ and $f a c$ $\operatorname{Ir}(\mathrm{ppy})_{3}\left(3.9 \mathrm{mg}, 6 \times 10^{-3} \mathrm{mmol}\right)$ in $\mathrm{CD}_{3} \mathrm{CN}(2.0 \mathrm{~mL})$. These two systems were bubbled with $\mathrm{Ar}$ for 15 minutes. Then, styrene $2(115 \mathrm{ul}, 1.0 \mathrm{mmol})$ were added into these tubes. One reaction system was tested by ${ }^{1} \mathrm{H}$ NMR directly. The other one was sealed and irradiated at room temperature by blue LEDs $(460 \mathrm{~nm})$ for 24 hours. When reaction was finished, the mixture was tested by ${ }^{1} \mathrm{H}$ NMR with 2,2-diphenylacetonitrile as internal standard. $60 \%$ 41, $40 \%$ free acetic anion and 100\% acetonitrile fragmentation from $\mathbf{1}$ were detected. These facts revealed an intriguing scission and reassembly of oxime ester into styrene. 


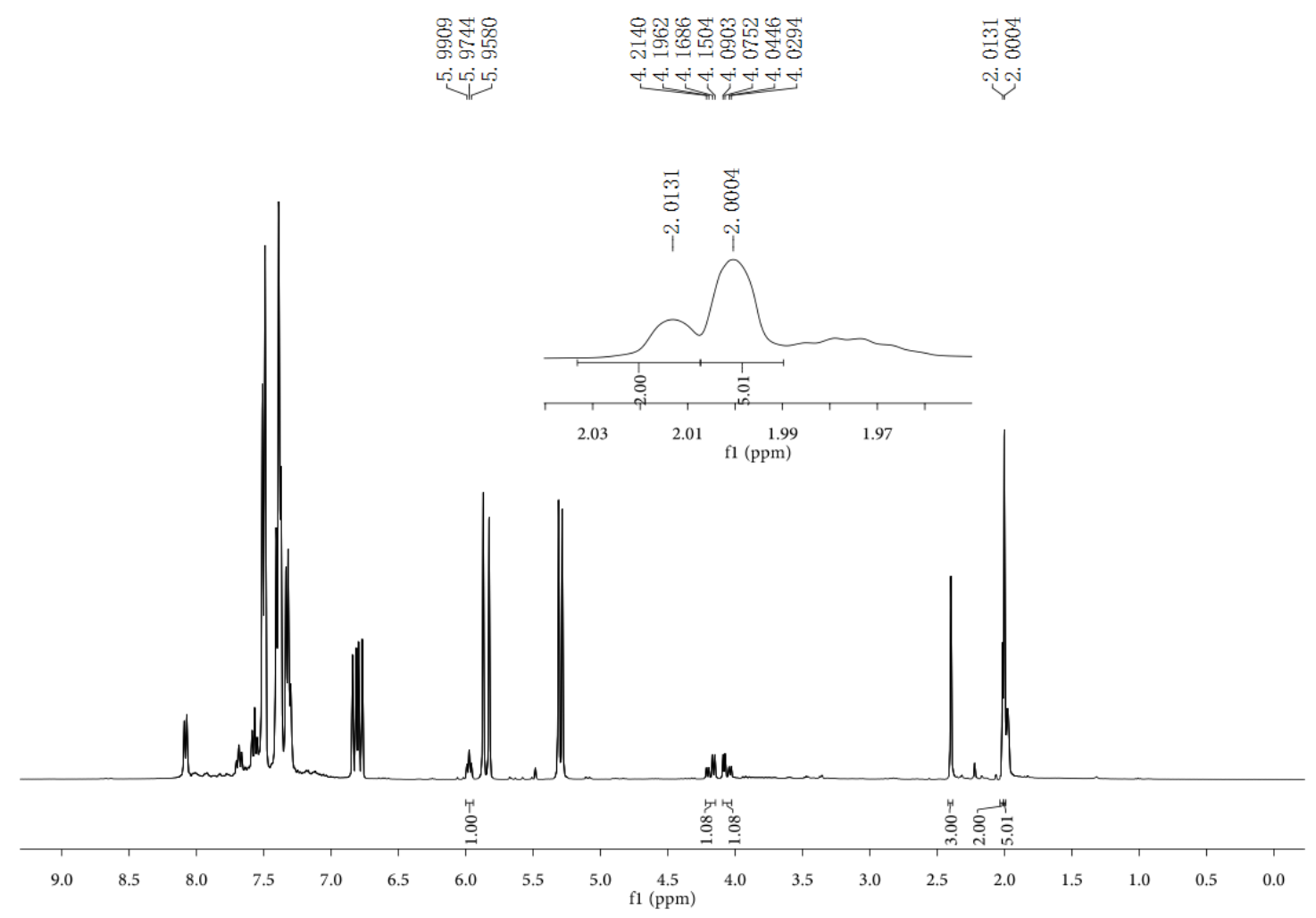

Figure S8. In situ ${ }^{1} \mathrm{H}$ NMR spectra of reaction mixture after $24 \mathrm{~h}$.

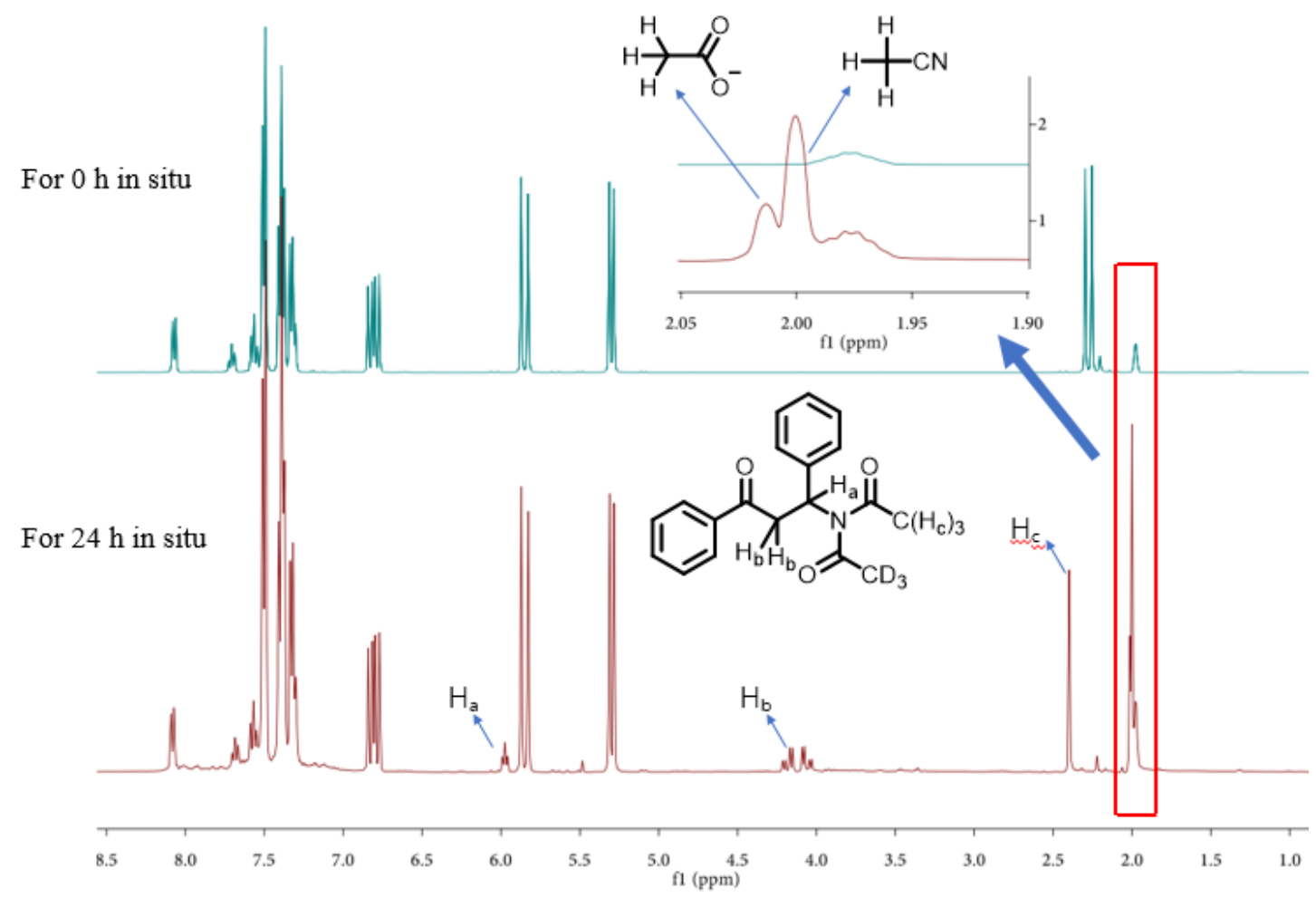

Figure S9. Comparison of in situ ${ }^{1} \mathrm{H}$ NMR spectra of reaction system before and after reaction. 


\section{c) ${ }^{18} \mathrm{O}$ labeled reaction for the reaction possibility of water:}

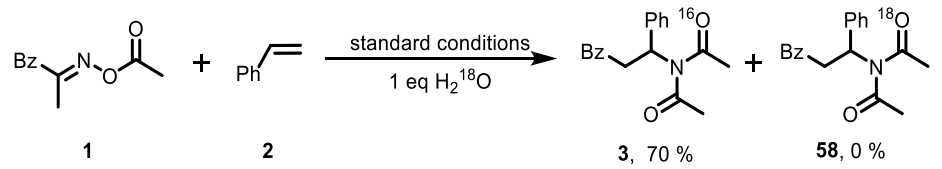

A $10 \mathrm{~mL}$ Pyrex tube equipped with a magnetic stir bar was charged with $1(0.2 \mathrm{mmol})$ and $f a c$ $\operatorname{Ir}(\mathrm{ppy})_{3}\left(3.9 \mathrm{mg}, 6 \times 10^{-3} \mathrm{mmol}\right)$ in $\mathrm{CH}_{3} \mathrm{CN}(2.0 \mathrm{~mL})$. This system was bubbled with Ar for 15 minutes. Then, styrene 2 (115ul, $1.0 \mathrm{mmol}$ ) were added into the tube. The tube was sealed and irradiated at room temperature by blue LEDs $(460 \mathrm{~nm})$ for 24 hours. When reaction was finished, the mixture was evaporated to remove the solvent and the residue was purified by flash chromatography on silica gel (petroleum ether/ethyl acetate) to afford the desired product 3 with $70 \%$ yield. The HRMS (ESI) examination of 3 (calcd for $\mathrm{C}_{19} \mathrm{H}_{19} \mathrm{NNaO}_{3}[\mathrm{M}+\mathrm{Na}]^{+} 332.14257$, found 332.1247) found no ${ }^{18} \mathrm{O}$-labeled product.

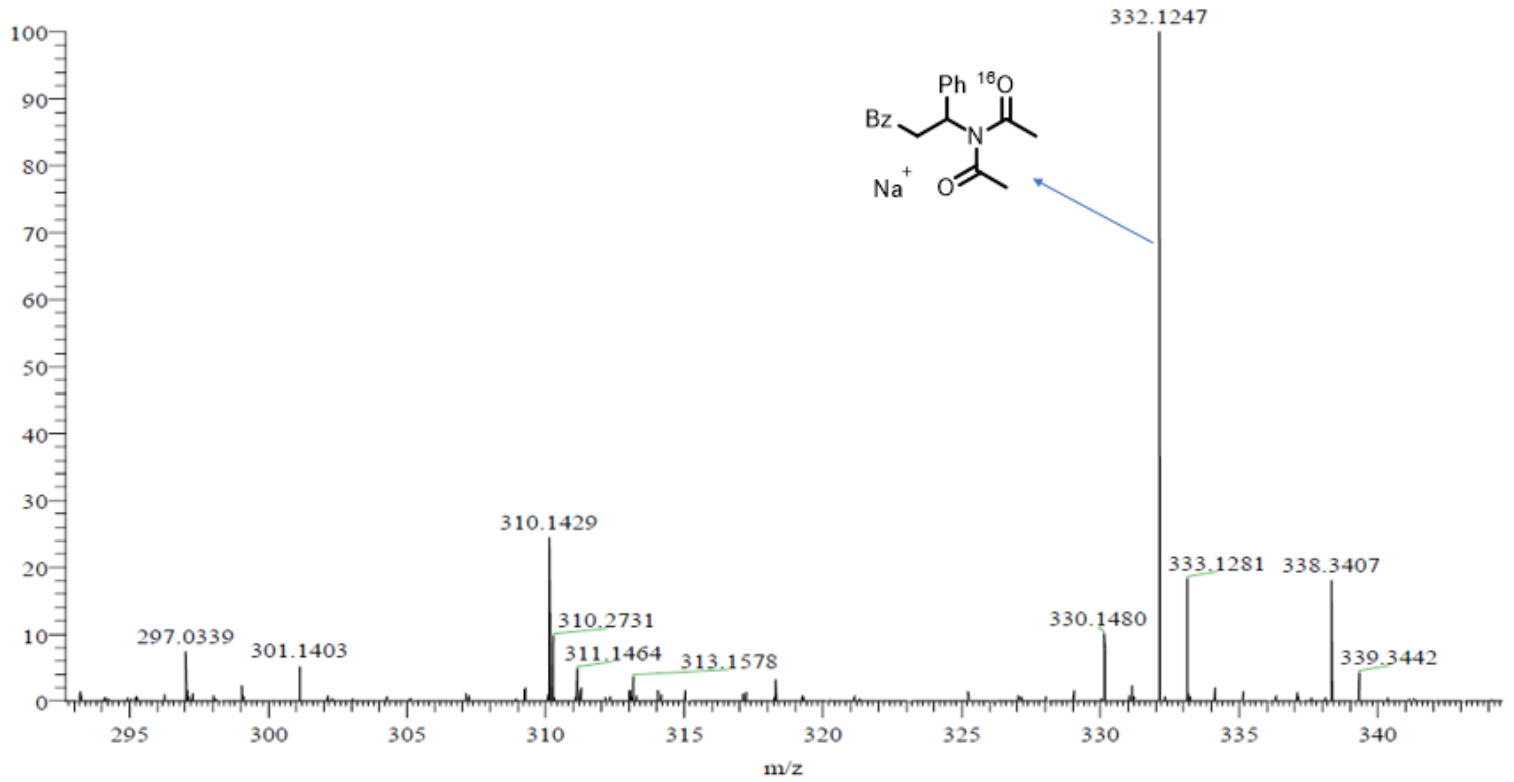

Figure S10. HRMS (ESI) examination of ${ }^{18} \mathrm{O}$-labeled reaction. 


\section{Characterization Data of All Products}

\section{$N$-acetyl- $N$-(3-oxo-1,3-diphenylpropyl)acetamide (3):}<smiles>CC(=O)N(C(C)=O)C(CC(=O)c1ccccc1)c1ccccc1</smiles>

The compound was prepared according to the general procedure for reaction of oxime ester and styrene with petroleum ether/ethyl acetate as eluent. Yield: $83 \%$ (51.3 mg, yellow solid). ${ }^{1} \mathrm{H}$ NMR $\left(400 \mathrm{MHz} \mathrm{CDCl}_{3}\right) \delta 8.01(\mathrm{~d}, J=7.7 \mathrm{~Hz}, 2 \mathrm{H}), 7.60(\mathrm{t}, J=7.2 \mathrm{~Hz}, 1 \mathrm{H}), 7.48(\mathrm{t}, J=7.6 \mathrm{~Hz}, 2 \mathrm{H}), 7.37$ $-7.26(\mathrm{~m}, 5 \mathrm{H}), 5.94-5.81(\mathrm{~m}, 1 \mathrm{H}), 4.26(\mathrm{dd}, J=18.1,7.8 \mathrm{~Hz}, 1 \mathrm{H}), 3.85(\mathrm{dd}, J=18.2,5.3 \mathrm{~Hz}, 1 \mathrm{H})$, $2.46(\mathrm{~s}, 6 \mathrm{H}) .{ }^{13} \mathrm{C} \mathrm{NMR}\left(101 \mathrm{MHz}, \mathrm{CDCl}_{3}\right) \delta 198.00,174.42,139.40,136.43,133.66,128.79,128.68$, $128.25,127.40,126.09,55.96,41.60,26.86$. HRMS (ESI): calcd for $\mathrm{C} 19 \mathrm{H} 19 \mathrm{NNaO} 3[\mathrm{M}+\mathrm{Na}]^{+}$ 332.1257 , found 332.1247 .

\section{$N$-acetyl- $N$-(3-oxo-1-phenyl-3-(o-tolyl)propyl)acetamide (4):}<smiles>CC(=O)N(C(C)=O)C(CC(=O)c1ccccc1C)c1ccccc1</smiles>

The compound was prepared according to the general procedure for reaction of oxime ester and styrene with petroleum ether/ethyl acetate as eluent. Yield: 46\% (30.0 mg, colorless oil). ${ }^{1} \mathrm{H}$ NMR $\left(400 \mathrm{MHz} \mathrm{CDCl}_{3}\right) \delta 7.71(\mathrm{~d}, \mathrm{~J}=7.7 \mathrm{~Hz}, 1 \mathrm{H}), 7.40(\mathrm{t}, J=7.4 \mathrm{~Hz}, 1 \mathrm{H}), 7.36-7.31(\mathrm{~m}, 2 \mathrm{H}), 7.30-$ $7.24(\mathrm{~m}, 5 \mathrm{H}), 5.91-5.80(\mathrm{~m}, 1 \mathrm{H}), 4.17(\mathrm{dd}, J=17.9,7.9 \mathrm{~Hz}, 1 \mathrm{H}), 3.76(\mathrm{dd}, J=17.9,5.4 \mathrm{~Hz}, 1 \mathrm{H})$, $2.48(\mathrm{~s}, 3 \mathrm{H}), 2.46(\mathrm{~s}, 6 \mathrm{H}) .{ }^{13} \mathrm{C} \mathrm{NMR}\left(101 \mathrm{MHz}, \mathrm{CDCl}_{3}\right) \delta 201.54,174.43,139.37,138.49,137.16$, 132.13, 131.90, 128.91, 128.69, 127.41, 126.12, 125.91, 56.16, 44.34, 26.88, 21.37. HRMS (ESI) : calcd for $\mathrm{C}_{20} \mathrm{H}_{21} \mathrm{NNaO}_{3}[\mathrm{M}+\mathrm{Na}]^{+} 346.1414$, found 346.1401 .

$N$-acetyl- $N$-(3-oxo-1-phenyl-3-(m-tolyl)propyl)acetamide (5):<smiles>CC(=O)N(C(C)=O)C(CC(=O)c1cccc(C)c1)c1ccccc1</smiles>

The compound was prepared according to the general procedure for reaction of oxime ester and styrene with petroleum ether/ethyl acetate as eluent. Yield: $61 \%$ (39.4 mg, white soild). ${ }^{1} \mathrm{H}$ NMR $\left(400 \mathrm{MHz} \mathrm{CDCl}_{3}\right) \delta 7.84-7.77(\mathrm{~m}, 2 \mathrm{H}), 7.43-7.26(\mathrm{~m}, 7 \mathrm{H}), 5.94-5.82(\mathrm{~m}, 1 \mathrm{H}), 4.23(\mathrm{dd}, J=$ 18.1, 7.7 Hz, 1H), $3.85(\mathrm{dd}, J=18.2,5.0 \mathrm{~Hz}, 1 \mathrm{H}), 2.46(\mathrm{~s}, 6 \mathrm{H}), 2.41(\mathrm{~s}, 3 \mathrm{H}) .{ }^{13} \mathrm{C} \mathrm{NMR}(101 \mathrm{MHz}$, $\left.\mathrm{CDCl}_{3}\right) \delta 198.15,174.42,139.45,138.62,136.43,134.43,128.77,128.67,127.37,126.07,125.47$, 55.96, 41.65, 26.87, 21.35. HRMS (ESI): calcd for $\mathrm{C}_{20} \mathrm{H}_{21} \mathrm{NNaO}_{3}[\mathrm{M}+\mathrm{Na}]^{+} 346.1414$, found 346.1402 .

$N$-acetyl- $N$-(3-oxo-1-phenyl-3-(p-tolyl)propyl)acetamide (6): 
<smiles>CC(=O)N(CC(=O)c1ccc(C)cc1)C(C)=O</smiles>

The compound was prepared according to the general procedure for reaction of oxime ester and styrene with petroleum ether/ethyl acetate as eluent. Yield: $61 \%$ (39.5 mg, colorless oil). ${ }^{1} \mathrm{H}$ NMR $\left(400 \mathrm{MHz}, \mathrm{CDCl}_{3}\right) \delta 8.02(\mathrm{~d}, J=8.1 \mathrm{~Hz}, 2 \mathrm{H}), 7.48-7.37(\mathrm{~m}, 7 \mathrm{H}), 6.05-5.95(\mathrm{~m}, 1 \mathrm{H}), 4.33(\mathrm{dd}, J$ $=18.0,7.7 \mathrm{~Hz}, 1 \mathrm{H}), 3.94(\mathrm{dd}, J=18.0,5.3 \mathrm{~Hz}, 1 \mathrm{H}), 2.57(\mathrm{~s}, 6 \mathrm{H}), 2.53(\mathrm{~s}, 3 \mathrm{H}) .{ }^{13} \mathrm{C} \mathrm{NMR}(101 \mathrm{MHz}$, $\left.\mathrm{CDCl}_{3}\right) \delta 197.58,174.43,144.59,139.49,133.97,129.46,128.66,128.39,127.35,126.06,56.00$, 41.44, 26.87, 21.71. HRMS (ESI): calcd for $\mathrm{C}_{20} \mathrm{H}_{21} \mathrm{NNa} 0_{3}[\mathrm{M}+\mathrm{Na}]^{+} 346.1414$, found 346.1402.

$N$-acetyl- $N$-(3-(4-chlorophenyl)-3-oxo-1-phenylpropyl)acetamide (7):<smiles>CC(=O)N(C(C)=O)C(CC(=O)c1ccc(Cl)cc1)c1ccccc1</smiles>

The compound was prepared according to the general procedure for reaction of oxime ester and styrene with petroleum ether/ethyl acetate as eluent. Yield: $56 \%$ (38.4 mg, pale yellow solid). ${ }^{1} \mathrm{H}$ $\operatorname{NMR}\left(400 \mathrm{MHz}, \mathrm{CDCl}_{3}\right) \delta 8.05(\mathrm{~d}, J=8.5 \mathrm{~Hz}, 2 \mathrm{H}), 7.56(\mathrm{~d}, J=8.5 \mathrm{~Hz}, 2 \mathrm{H}), 7.48-7.43(\mathrm{~m}, 2 \mathrm{H})$, $7.42-7.37$ (m, 3H), 5.97 (dd, $J=7.3,5.8 \mathrm{~Hz}, 1 \mathrm{H}), 4.37$ (dd, $J=18.1,7.9 \mathrm{~Hz}, 1 \mathrm{H}), 3.91(\mathrm{dd}, J=$ 18.1, $5.3 \mathrm{~Hz}, 1 \mathrm{H}), 2.56(\mathrm{~s}, 6 \mathrm{H}) .{ }^{13} \mathrm{C}$ NMR $\left(101 \mathrm{MHz}, \mathrm{CDCl}_{3}\right) \delta$ 196.83, 174.40, 140.19, 139.19, $134.71,129.67,129.11,128.72,127.50,126.08,55.93,41.55,26.85$. HRMS (ESI): calcd for $\mathrm{C}_{19} \mathrm{H}_{18} \mathrm{ClNNaO}_{3}[\mathrm{M}+\mathrm{Na}]^{+}$366.0867, found 366.0857.

$N$-acetyl- $N$-(3-(4-methoxyphenyl)-3-oxo-1-phenylpropyl)acetamide (8):<smiles>COc1ccc(C(=O)C(C(C)=O)N(C(C)=O)C(C)=O)cc1</smiles>

The compound was prepared according to the general procedure for reaction of oxime ester and styrene with petroleum ether/ethyl acetate as eluent. Yield: 43\% (29.2 mg, white solid). ${ }^{1} \mathrm{H}$ NMR $\left(400 \mathrm{MHz}, \mathrm{CDCl}_{3}\right) \delta 7.99(\mathrm{~d}, J=7.9 \mathrm{~Hz}, 2 \mathrm{H}), 7.37-7.25(\mathrm{~m}, 5 \mathrm{H}), 6.95(\mathrm{~d}, J=7.8 \mathrm{~Hz}, 2 \mathrm{H}), 5.93-$ $5.83(\mathrm{~m}, 1 \mathrm{H}), 4.19(\mathrm{dd}, J=17.8,7.7 \mathrm{~Hz}, 1 \mathrm{H}), 3.88(\mathrm{~s}, 3 \mathrm{H}), 3.79(\mathrm{dd}, J=17.8,5.1 \mathrm{~Hz}, 1 \mathrm{H}), 2.45(\mathrm{~s}$, 6H). ${ }^{13} \mathrm{C} \mathrm{NMR}\left(101 \mathrm{MHz}, \mathrm{CDCl}_{3}\right) \delta 196.45,174.48,163.98,139.55,130.63,129.53,128.66,127.33$, 126.05, 113.94, 56.13, 55.55, 41.16, 26.86. HRMS (ESI): calcd for $\mathrm{C}_{20} \mathrm{H}_{21} \mathrm{NNaO}_{4}[\mathrm{M}+\mathrm{Na}]^{+}$ 362.1363 , found 362.1351 .

$N$-acetyl- $N$-(3-(furan-2-yl)-3-oxo-1-phenylpropyl)acetamide(9):<smiles>CC(=O)C(c1ccccc1)N(C(C)=O)C(C)=O</smiles> 
The compound was prepared according to the general procedure for reaction of oxime ester and styrene with petroleum ether/ethyl acetate as eluent. Yield: 86\% (51.4 mg, white solid). ${ }^{1} \mathrm{H}$ NMR $\left(400 \mathrm{MHz}, \mathrm{CDCl}_{3}\right) \delta 7.63(\mathrm{~s}, 1 \mathrm{H}), 7.37-7.26(\mathrm{~m}, 6 \mathrm{H}), 6.63-6.53(\mathrm{~m}, 1 \mathrm{H}), 5.85(\mathrm{dd}, J=8.2,5.4$ $\mathrm{Hz}, 1 \mathrm{H}), 4.11(\mathrm{dd}, J=17.5,8.4 \mathrm{~Hz}, 1 \mathrm{H}), 3.70(\mathrm{dd}, J=17.5,5.3 \mathrm{~Hz}, 1 \mathrm{H}), 2.44(\mathrm{~s}, 6 \mathrm{H}) .{ }^{13} \mathrm{C}$ NMR $\left(101 \mathrm{MHz}, \mathrm{CDCl}_{3}\right) \delta 186.70,174.32,152.23,147.09,139.17,128.67,127.45,126.04,118.32$, 112.51, 55.55, 41.08, 26.84. HRMS (ESI): calcd for $\mathrm{C}_{17} \mathrm{H}_{17} \mathrm{NaNO}_{4}[\mathrm{M}+\mathrm{H}]^{+} 322.1050$, found 322.1039 .

\section{$N$-acetyl- $N$-(3-oxo-1-phenylbutyl)acetamide (10):}<smiles>CC(=O)CC(C(C)=O)(C(C)=O)C(C)=O</smiles>

The compound was prepared according to the general procedure for reaction of oxime ester and styrene with petroleum ether/ethyl acetate as eluent. Yield: 60\% (29.7 mg, pale yellow solid). ${ }^{1} \mathrm{H}$ $\operatorname{NMR}\left(400 \mathrm{MHz}, \mathrm{CDCl}_{3}\right) \delta 7.33(\mathrm{dd}, \mathrm{J}=7.4,7.6 \mathrm{~Hz}, 2 \mathrm{H}), 7.29-2.26(\mathrm{~m}, 1 \mathrm{H}), 7.24-7.20(\mathrm{~m}, 2 \mathrm{H})$ $5.73-5.60(\mathrm{t}, J=6.5 \mathrm{~Hz}, 1 \mathrm{H}), 3.67(\mathrm{dd}, J=18.4,7.8 \mathrm{~Hz}, 1 \mathrm{H}), 3.36(\mathrm{dd}, J=18.4,5.3 \mathrm{~Hz}, 1 \mathrm{H}), 2.43$ (s, 6H), 2.22 (s, 3H). ${ }^{13} \mathrm{C} \mathrm{NMR}\left(101 \mathrm{MHz}, \mathrm{CDCl}_{3}\right) \delta 206.46,174.37,139.16,128.67,127.44,126.03$, 55.31, 46.40, 30.31, 26.83. HRMS (ESI): calcd for $\mathrm{C}_{14} \mathrm{H}_{17} \mathrm{NaNO}_{3}[\mathrm{M}+\mathrm{Na}]^{+} 270.1101$, found 270.1095 .

\section{$N$-acetyl- $N$-(3-oxo-1-phenylpentyl)acetamide (11):}<smiles>CCC(=O)C(C(C)=O)N(C(C)=O)C(C)=O</smiles>

The compound was prepared according to the general procedure for reaction of oxime ester and styrene with petroleum ether/ethyl acetate as eluent. Yield: $44 \%$ (23.0 mg, white solid). ${ }^{1} \mathrm{H}$ NMR $\left(400 \mathrm{MHz}, \mathrm{CDCl}_{3}\right) \delta 7.33(\mathrm{t}, J=7.4 \mathrm{~Hz}, 2 \mathrm{H}), 7.28-7.20(\mathrm{~m}, 3 \mathrm{H}), 5.77-5.61(\mathrm{~m}, 1 \mathrm{H}), 3.66(\mathrm{dd}, J$ $=18.1,8.0 \mathrm{~Hz}, 1 \mathrm{H}), 3.31(\mathrm{dd}, J=18.1,5.1 \mathrm{~Hz}, 1 \mathrm{H}), 2.50(\mathrm{q}, J=14.0,7.1 \mathrm{~Hz}, 2 \mathrm{H}), 2.43(\mathrm{~s}, 6 \mathrm{H})$, $1.08(\mathrm{t}, J=7.2 \mathrm{~Hz}, 3 \mathrm{H}) .{ }^{13} \mathrm{C} \mathrm{NMR}\left(101 \mathrm{MHz}, \mathrm{CDCl}_{3}\right) \delta 209.37,174.39,139.26,128.66,127.41$, 126.04, 55.46, 45.12, 36.38, 26.82, 7.68. HRMS (ESI): calcd for $\mathrm{C}_{15} \mathrm{H}_{19} \mathrm{NNaO}_{3}[\mathrm{M}+\mathrm{Na}]^{+} 284.1257$, found 284.1249 .

$N$-acetyl- $N$-(3-oxo-1-phenylheptyl)acetamide (12):

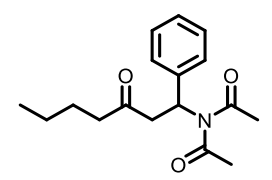

The compound was prepared according to the general procedure for reaction of oxime ester and styrene with petroleum ether/ethyl acetate as eluent. Yield: $66 \%\left(38.2 \mathrm{mg}\right.$, pale yellow solid). ${ }^{1} \mathrm{H}$ $\operatorname{NMR}\left(400 \mathrm{MHz}, \mathrm{CDCl}_{3}\right) \delta 7.33(\mathrm{t}, J=7.4 \mathrm{~Hz}, 2 \mathrm{H}), 7.28-7.20(\mathrm{~m}, 3 \mathrm{H}), 5.78-5.57(\mathrm{~m}, 1 \mathrm{H}), 3.67$ $(\mathrm{dd}, J=18.1,8.1 \mathrm{~Hz}, 1 \mathrm{H}), 3.30(\mathrm{dd}, J=18.2,5.1 \mathrm{~Hz}, 1 \mathrm{H}), 2.59-2.31(\mathrm{~m}, 8 \mathrm{H}), 1.63-1.52(\mathrm{~m}, 2 \mathrm{H})$, 
$1.36-1.25(\mathrm{~m}, 2 \mathrm{H}), 0.91(\mathrm{t}, J=7.3 \mathrm{~Hz}, 3 \mathrm{H}) .{ }^{13} \mathrm{C} \mathrm{NMR}\left(101 \mathrm{MHz}, \mathrm{CDCl}_{3}\right) \delta 209.10,174.39,139.27$, 128.65, 127.40, 126.04, 55.46, 45.45, 42.97, 26.82, 25.79, 22.28, 13.81. HRMS (ESI): calcd for $\mathrm{C}_{17} \mathrm{H}_{23} \mathrm{NNaO}{ }_{3}[\mathrm{M}+\mathrm{Na}]^{+} 312.1570$, found 312.1560 .

$N$-acetyl- $N$-(5-methyl-3-oxo-1-phenylhexyl)acetamide (13):

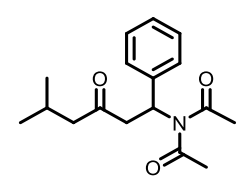

The compound was prepared according to the general procedure for reaction of oxime ester and styrene with petroleum ether/ethyl acetate as eluent. Yield: $73 \%$ (42.2 mg, pale yellow oil). ${ }^{1} \mathrm{H}$ NMR $\left(400 \mathrm{MHz}, \mathrm{CDCl}_{3}\right) \delta 7.33(\mathrm{t}, J=7.6 \mathrm{~Hz}, 2 \mathrm{H}), 7.27-7.21(\mathrm{~m}, 3 \mathrm{H}), 5.66(\mathrm{dd}, J=7.7,5.6 \mathrm{~Hz}, 1 \mathrm{H})$, $3.68(\mathrm{dd}, J=18.2,8.2 \mathrm{~Hz}, 1 \mathrm{H}), 3.26(\mathrm{dd}, J=18.2,5.2 \mathrm{~Hz}, 1 \mathrm{H}), 2.44(\mathrm{~s}, 6 \mathrm{H}), 2.34(\mathrm{~d}, J=7.0 \mathrm{~Hz}$, $2 \mathrm{H}), 2.21-2.10(\mathrm{~m}, 1 \mathrm{H}), 0.92(\mathrm{~d}, J=6.6 \mathrm{~Hz}, 6 \mathrm{H}) .{ }^{13} \mathrm{C} \mathrm{NMR}\left(101 \mathrm{MHz}, \mathrm{CDCl}_{3}\right) \delta 208.72,174.52$, 139.55, 128.80, 127.56, 126.31, 55.70, 52.42, 46.22, 26.89, 24.76, 22.70, 22.61. HRMS (ESI): calcd for $\mathrm{C}_{17} \mathrm{H}_{23} \mathrm{NNaO}_{3}[\mathrm{M}+\mathrm{Na}]^{+}$312.1570, found 312.1562.

$N$-acetyl- $N$-(5-methyl-3-oxo-1-phenylheptyl)acetamide (14):

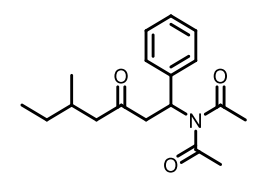

The compound was prepared according to the general procedure for reaction of oxime ester and styrene with petroleum ether/ethyl acetate as eluent. Yield: $47 \%$ (28.5 mg, pale yellow oil). ${ }^{1} \mathrm{H}$ NMR $\left(400 \mathrm{MHz}, \mathrm{CDCl}_{3}\right) \delta 7.33(\mathrm{t}, \mathrm{J}=7.5 \mathrm{~Hz}, 2 \mathrm{H}), 7.26-7.21(\mathrm{~m}, 3 \mathrm{H}), 5.77-5.59(\mathrm{~m}, 1 \mathrm{H}), 3.75-3.62$ $(\mathrm{m}, 1 \mathrm{H}), 3.31-3.22(\mathrm{~m}, 1 \mathrm{H}), 2.57-2.32(\mathrm{~m}, 7 \mathrm{H}), 2.30-2.23(\mathrm{~m}, 1 \mathrm{H}), 1.97-1.87(\mathrm{~m}, 1 \mathrm{H}), 1.36$ $-1.27(\mathrm{~m}, 1 \mathrm{H}), 1.24-1.13(\mathrm{~m}, 1 \mathrm{H}), 0.91-0.85(\mathrm{~m}, 6 \mathrm{H}) .{ }^{13} \mathrm{C} \mathrm{NMR}\left(101 \mathrm{MHz}, \mathrm{CDCl}_{3}\right) \delta 209.01$, 208.96, 174.40, 139.29, 139.27, 128.65, 127.41, 126.08, 55.52, 55.46, 50.37, 50.33, 45.99, 45.94, $30.82,30.80,29.52,29.41,26.83,19.35,19.33,11.31,11.29$. HRMS (ESI): calcd for $\mathrm{C}_{18} \mathrm{H}_{25} \mathrm{NNaO}_{3}$ $[\mathrm{M}+\mathrm{Na}]^{+} 326.1727$, found 326.1716 .

$N$-acetyl- $N$-(3-oxo-1,3-diphenylpropyl)propionamide (15):<smiles>CCC(=O)N(C(C)=O)C(CC(=O)c1ccccc1)c1ccccc1</smiles>

The compound was prepared according to the general procedure for reaction of oxime ester and styrene with petroleum ether/ethyl acetate as eluent. Yield: 84\% (54.3 mg, white solid). ${ }^{1} \mathrm{H}$ NMR $\left(400 \mathrm{MHz}, \mathrm{CDCl}_{3}\right) \delta 7.93(\mathrm{~d}, J=7.7 \mathrm{~Hz}, 2 \mathrm{H}), 7.52(\mathrm{t}, J=7.3 \mathrm{~Hz}, 1 \mathrm{H}), 7.40(\mathrm{t}, J=7.7 \mathrm{~Hz}, 2 \mathrm{H}), 7.28$ $-7.17(\mathrm{~m}, 5 \mathrm{H}), 5.91-5.77(\mathrm{~m}, 1 \mathrm{H}), 4.12(\mathrm{dd}, J=18.1,7.5 \mathrm{~Hz}, 1 \mathrm{H}), 3.81(\mathrm{dd}, J=18.1,5.5 \mathrm{~Hz}, 1 \mathrm{H})$, $2.80(\mathrm{dq}, J=17.0,7.3 \mathrm{~Hz}, 1 \mathrm{H}), 2.61(\mathrm{dq}, J=17.0,7.3 \mathrm{~Hz}, 1 \mathrm{H}), 2.37(\mathrm{~s}, 3 \mathrm{H}), 1.03(\mathrm{t}, J=7.3 \mathrm{~Hz}, 3 \mathrm{H})$. ${ }^{13} \mathrm{C}$ NMR $\left(101 \mathrm{MHz}, \mathrm{CDCl}_{3}\right) \delta 198.01,178.27,174.42,139.56,136.43,133.65,128.78,128.66$, 
128.26, 127.33, 126.05, 55.46, 41.87, 31.68, 26.93, 9.29. HRMS (ESI): calcd for $\mathrm{C}_{20} \mathrm{H}_{21} \mathrm{NNaO}_{3}$ $[\mathrm{M}+\mathrm{Na}]^{+} 346.1414$, found 346.1404 .

$N$-acetyl- $N$-(3-oxo-1,3-diphenylpropyl)hexanamide (16):

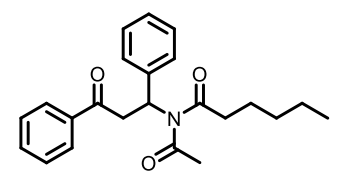

The compound was prepared according to the general procedure for reaction of oxime ester and styrene with petroleum ether/ethyl acetate as eluent. Yield: $56 \%$ (29.7 mg, colorless oil). ${ }^{1} \mathrm{H}$ NMR $\left(400 \mathrm{MHz}, \mathrm{CDCl}_{3}\right) \delta 8.00(\mathrm{~d}, J=7.7 \mathrm{~Hz}, 2 \mathrm{H}), 7.59(\mathrm{t}, J=7.2 \mathrm{~Hz}, 1 \mathrm{H}), 7.48(\mathrm{t}, J=7.4 \mathrm{~Hz}, 2 \mathrm{H}), 7.34$ $-7.24(\mathrm{~m}, 5 \mathrm{H}), 6.03-5.84(\mathrm{~m}, 1 \mathrm{H}), 4.20(\mathrm{dd}, J=18.1,7.4 \mathrm{~Hz}, 1 \mathrm{H}), 3.88(\mathrm{dd}, J=18.1,5.3 \mathrm{~Hz}, 1 \mathrm{H})$, $2.81(\mathrm{dt}, J=16.4 \mathrm{~Hz}, 7.4 \mathrm{~Hz}, 1 \mathrm{H}), 2.68(\mathrm{dt}, J=16.4 \mathrm{~Hz}, 7.4 \mathrm{~Hz}, 1 \mathrm{H}), 2.45(\mathrm{~s}, 3 \mathrm{H}), 1.59$ (tt, $J=7.0$ $\mathrm{Hz}, 2 \mathrm{H}), 1.31-1.18(\mathrm{~m}, 4 \mathrm{H}), 0.84(\mathrm{t}, J=6.6 \mathrm{~Hz}, 3 \mathrm{H}) .{ }^{13} \mathrm{C} \mathrm{NMR}\left(101 \mathrm{MHz}, \mathrm{CDCl}_{3}\right) \delta 198.10$, $177.86,174.57,139.84,136.77,133.73,128.92,128.80,128.43,127.49,126.36,55.77,42.07,38.48$, 31.42, 26.89, 24.91, 22.55, 13.99. HRMS (ESI): calcd for $\mathrm{C}_{23} \mathrm{H}_{27} \mathrm{NNaO}_{3}[\mathrm{M}+\mathrm{Na}]^{+} 388.1883$, found 388.1873 .

\section{$N$-acetyl- $N$-(3-oxo-1,3-diphenylpropyl)tridecanamide (17):}

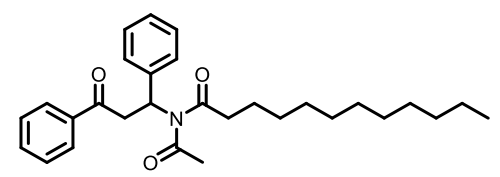

The compound was prepared according to the general procedure for reaction of oxime ester and styrene with petroleum ether/ethyl acetate as eluent. Yield: $60 \%$ (59.9 mg, colorless oil). ${ }^{1} \mathrm{H}$ NMR $\left(400 \mathrm{MHz}, \mathrm{CDCl}_{3}\right) \delta 8.00(\mathrm{~d}, J=7.7 \mathrm{~Hz}, 2 \mathrm{H}), 7.59(\mathrm{t}, J=7.2 \mathrm{~Hz}, 1 \mathrm{H}), 7.47$ (t, $\left.J=7.4 \mathrm{~Hz}, 2 \mathrm{H}\right), 7.36$ $-7.24(\mathrm{~m}, 5 \mathrm{H}), 5.97-5.84(\mathrm{~m}, 1 \mathrm{H}), 4.20(\mathrm{dd}, J=18.1,7.4 \mathrm{~Hz}, 1 \mathrm{H}), 3.88(\mathrm{dd}, J=18.1,5.3 \mathrm{~Hz}, 1 \mathrm{H})$, $2.80(\mathrm{dt}, J=16.4 \mathrm{~Hz}, 7.4 \mathrm{~Hz}, 1 \mathrm{H}), 2.65(\mathrm{dt}, J=16.4 \mathrm{~Hz}, 7.4 \mathrm{~Hz}, 1 \mathrm{H}), 2.44(\mathrm{~s}, 3 \mathrm{H}), 1.63-1.54(\mathrm{~m}$, $2 \mathrm{H}), 1.45-1.02(\mathrm{~m}, 18 \mathrm{H}), 0.88(\mathrm{t}, J=6.4 \mathrm{~Hz}, 3 \mathrm{H}) .{ }^{13} \mathrm{C} \mathrm{NMR}\left(101 \mathrm{MHz}, \mathrm{CDCl}_{3}\right) \delta 197.96,177.70$, 174.44, 139.59, 136.47, 133.63, 128.77, 128.65, 128.27, 127.33, 126.12, 55.49, 41.83, 38.34, 31.93, 29.61, 29.60, 29.46, 29.39, 29.34, 29.09, 26.85, 25.06, 22.70, 14.13. HRMS (ESI): calcd for $\mathrm{C}_{29} \mathrm{H}_{39} \mathrm{NNaO}_{3}[\mathrm{M}+\mathrm{Na}]^{+}$472.2822, found 472.2808 .

\section{$N$-acetyl- $N$-(3-oxo-1,3-diphenylpropyl)tridecanamide (18):}

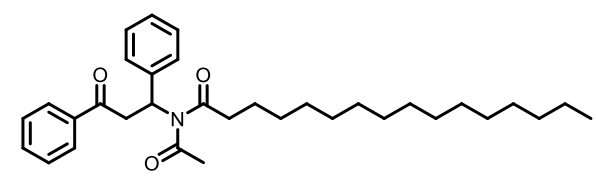

The compound was prepared according to the general procedure for reaction of oxime ester and styrene with petroleum ether/ethyl acetate as eluent. Yield: $54 \%$ (54.6 mg, white solid). ${ }^{1} \mathrm{H}$ NMR $\left(400 \mathrm{MHz}, \mathrm{CDCl}_{3}\right) \delta 8.00$ (d, $\left.J=7.4 \mathrm{~Hz}, 2 \mathrm{H}\right), 7.59$ (t, $\left.J=7.4 \mathrm{~Hz}, 1 \mathrm{H}\right), 7.47$ (t, $\left.J=7.7 \mathrm{~Hz}, 2 \mathrm{H}\right), 7.36$ $-7.22(\mathrm{~m}, 5 \mathrm{H}), 5.98-5.83(\mathrm{~m}, 1 \mathrm{H}), 4.20(\mathrm{dd}, J=18.1,7.5 \mathrm{~Hz}, 1 \mathrm{H}), 3.88(\mathrm{dd}, J=18.1,5.6 \mathrm{~Hz}, 1 \mathrm{H})$, $2.86-2.75(\mathrm{~m}, 1 \mathrm{H}), 2.70-2.60(\mathrm{~m}, 1 \mathrm{H}), 2.44(\mathrm{~s}, 3 \mathrm{H}), 1.62-1.54(\mathrm{~m}, 2 \mathrm{H}), 1.29-1.18(\mathrm{~m}, 24 \mathrm{H})$, 
$0.88(\mathrm{t}, J=6.8 \mathrm{~Hz}, 3 \mathrm{H}) .{ }^{13} \mathrm{C}$ NMR $(101 \mathrm{MHz}, \mathrm{CDCl} 3) \delta 197.94,177.68,174.42,139.57,136.45$, 133.62, 128.76, 128.64, 128.26, 127.32, 126.10, 55.48, 41.83, 38.34, 31.94, 29.71, 29.69, 29.67, 29.61, 29.46, 29.40, 29.38, 29.10, 26.85, 25.06, 22.71, 14.13. HRMS (ESI): calcd for $\mathrm{C}_{33} \mathrm{H}_{47} \mathrm{NNaO}_{3}$ $[\mathrm{M}+\mathrm{Na}]^{+} 528.3448$, found 528.3428 .

\section{Ethyl 4-oxo-4-( $N$-(3-oxo-1,3-diphenylpropyl)acetamido)butanoate (19):}<smiles>CCOC(=O)CCC(=O)N(CC(=O)c1ccccc1)C(C)=O</smiles>

The compound was prepared according to the general procedure for reaction of oxime ester and styrene with petroleum ether/ethyl acetate as eluent. Yield: 70\% (55.3 mg, white solid). ${ }^{1} \mathrm{H}$ NMR $\left(400 \mathrm{MHz}, \mathrm{CDCl}_{3}\right) \delta 8.00(\mathrm{~d}, J=7.7 \mathrm{~Hz}, 2 \mathrm{H}), 7.59(\mathrm{t}, J=7.2 \mathrm{~Hz}, 1 \mathrm{H}), 7.47(\mathrm{t}, J=7.5 \mathrm{~Hz}, 2 \mathrm{H}), 7.36$ $-7.23(\mathrm{~m}, 5 \mathrm{H}), 6.00-5.86(\mathrm{t}, 1 \mathrm{H}), 4.22(\mathrm{dd}, J=18.3,7.3 \mathrm{~Hz}, 1 \mathrm{H}), 4.07(\mathrm{q}, J=7.0 \mathrm{~Hz}, 2 \mathrm{H}), 3.90$ $(\mathrm{dd}, J=18.3,5.2 \mathrm{~Hz}, 1 \mathrm{H}), 3.17(\mathrm{dt}, J=17.2,5.9 \mathrm{~Hz}, 1 \mathrm{H}), 3.04(\mathrm{dt}, J=13.2,6.3 \mathrm{~Hz}, 1 \mathrm{H}), 2.74-$ $2.56(\mathrm{~m}, 2 \mathrm{H}), 2.44(\mathrm{~s}, 3 \mathrm{H}), 1.21(\mathrm{t}, J=7.1 \mathrm{~Hz}, 3 \mathrm{H}) .{ }^{13} \mathrm{C} \mathrm{NMR}\left(101 \mathrm{MHz}, \mathrm{CDCl}_{3}\right) \delta$ 197.98, 176.08, $174.34,172.59,139.31,136.44,133.61,128.74,128.66,128.27,127.29,125.94,60.60,55.34,41.71$, $33.28,29.45,26.72,14.18$. HRMS (ESI): calcd for $\mathrm{C}_{23} \mathrm{H}_{25} \mathrm{NNaO}_{5}[\mathrm{M}+\mathrm{Na}]^{+} 418.1625$, found 418.1608 .

$N$-acetyl- $N$-(3-oxo-1,3-diphenylpropyl)cyclopropanecarboxamide (20):<smiles>CC(=O)N1C(=O)CC(c2ccccc2)C(c2ccccc2)CC1=O</smiles>

The compound was prepared according to the general procedure for reaction of oxime ester and styrene with petroleum ether/ethyl acetate as eluent. Yield: 53\% (35.5 mg, white solid). ${ }^{1} \mathrm{H}$ NMR $\left(400 \mathrm{MHz}, \mathrm{CDCl}_{3}\right) \delta 8.01(\mathrm{~d}, J=7.5 \mathrm{~Hz}, 2 \mathrm{H}), 7.59(\mathrm{t}, J=7.2 \mathrm{~Hz}, 1 \mathrm{H}), 7.48(\mathrm{t}, J=7.5 \mathrm{~Hz}, 2 \mathrm{H}), 7.40$ $-7.26(\mathrm{~m}, 5 \mathrm{H}), 6.25-6.11(\mathrm{~m}, 1 \mathrm{H}), 4.22(\mathrm{dd}, J=17.9,7.6 \mathrm{~Hz}, 1 \mathrm{H}), 3.88(\mathrm{dd}, J=17.9,5.6 \mathrm{~Hz}, 1 \mathrm{H})$, $2.39(\mathrm{~s}, 3 \mathrm{H}), 2.14-2.05(\mathrm{~m}, 1 \mathrm{H}), 1.12(\mathrm{~m}, 2 \mathrm{H}), 0.96(\mathrm{~m}, 2 \mathrm{H}) .{ }^{13} \mathrm{C} \mathrm{NMR}(101 \mathrm{MHz}, \mathrm{CDCl} 3) \delta$ 197.82, 179.01, 173.61, 139.66, 136.55, 133.53, 128.74, 128.58, 128.25, 127.33, 126.52, 55.43, 41.74, 26.63, 17.11, 11.17, 11.06. HRMS (ESI): calcd for $\mathrm{C}_{21} \mathrm{H}_{21} \mathrm{NNaO}_{3}[\mathrm{M}+\mathrm{Na}]^{+} 358.1414$, found 358.1402 .

\section{$N$-acetyl- $N$-(3-oxo-1,3-diphenylpropyl)cyclobutanecarboxamide (21):}<smiles>CC(=O)N(C(=O)C1CC1)C(CC(=O)c1ccccc1)c1ccccc1</smiles>

The compound was prepared according to the general procedure for reaction of oxime ester and styrene with petroleum ether/ethyl acetate as eluent. Yield: 78\% (54.4 mg, white solid). ${ }^{1} \mathrm{H}$ NMR $\left(400 \mathrm{MHz}, \mathrm{CDCl}_{3}\right) \delta 8.01(\mathrm{~d}, J=7.6 \mathrm{~Hz}, 2 \mathrm{H}), 7.60(\mathrm{t}, J=7.3 \mathrm{~Hz}, 1 \mathrm{H}), 7.48(\mathrm{t}, J=7.5 \mathrm{~Hz}, 2 \mathrm{H}), 7.35$ $-7.29(\mathrm{~m}, 2 \mathrm{H}), 7.26-7.21(\mathrm{~m}, 3 \mathrm{H}), 5.88-5.77(\mathrm{~m}, 1 \mathrm{H}), 4.10-3.93(\mathrm{~m}, 2 \mathrm{H}), 3.69$ (p, J = 8.5 Hz, 
$1 \mathrm{H}), 2.43(\mathrm{~s}, 3 \mathrm{H}), 2.30-2.10(\mathrm{~m}, 4 \mathrm{H}), 1.98-1.85(\mathrm{~m}, 1 \mathrm{H}), 1.77(\mathrm{t}, J=9.4 \mathrm{~Hz}, 1 \mathrm{H}) .{ }^{13} \mathrm{C} \mathrm{NMR}(101$ $\left.\mathrm{MHz}, \mathrm{CDCl}_{3}\right) \delta 197.86,179.25,174.23,139.66,136.41,133.64,128.77,128.60,128.27,127.23$, 125.96, 55.17, 42.19, 41.53, 26.80, 25.70, 25.60, 17.55. HRMS (ESI): calcd for $\mathrm{C}_{22} \mathrm{H}_{23} \mathrm{NNaO}_{3}$ $[\mathrm{M}+\mathrm{Na}]^{+} 372.1570$, found 372.1559 .

$N$-acetyl- $N$-(3-oxo-1,3-diphenylpropyl)cyclopentanecarboxamide (22):<smiles>CC(=O)C(CC(=O)c1ccccc1)(C(C)=O)C(=O)C1CCCC1</smiles>

The compound was prepared according to the general procedure for reaction of oxime ester and styrene with petroleum ether/ethyl acetate as eluent. Yield: 55\% (39.9 mg, white solid). ${ }^{1} \mathrm{H}$ NMR $\left(400 \mathrm{MHz}, \mathrm{CDCl}_{3}\right) \delta 7.94(\mathrm{~d}, J=7.6 \mathrm{~Hz}, 2 \mathrm{H}), 7.53(\mathrm{t}, J=7.2 \mathrm{~Hz}, 1 \mathrm{H}), 7.41(\mathrm{t}, J=7.4 \mathrm{~Hz}, 2 \mathrm{H}), 7.29$ $-7.16(\mathrm{~m}, 5 \mathrm{H}), 6.04-5.78(\mathrm{~m}, 1 \mathrm{H}), 4.00(\mathrm{dd}, J=18.1,6.7 \mathrm{~Hz}, 1 \mathrm{H}), 3.90(\mathrm{dd}, J=18.1,6.0 \mathrm{~Hz}, 1 \mathrm{H})$, $3.36-3.13(\mathrm{~m}, 1 \mathrm{H}), 2.36(\mathrm{~s}, 3 \mathrm{H}), 1.88-1.72(\mathrm{~m}, 2 \mathrm{H}), 1.72-1.55(\mathrm{~m}, 4 \mathrm{H}), 1.52-1.41(\mathrm{~m}, 2 \mathrm{H})$. ${ }^{13} \mathrm{C}$ NMR $\left(101 \mathrm{MHz}, \mathrm{CDCl}_{3}\right) \delta 197.79,182.01,174.45,139.66,136.44,133.61,128.76,128.60$, 128.26, 127.29, 126.20, 55.35, 46.55, 42.18, 31.33, 31.12, 26.61, 26.14, 26.09. HRMS (ESI): calcd for $\mathrm{C}_{23} \mathrm{H}_{25} \mathrm{NNaO}_{3}[\mathrm{M}+\mathrm{Na}]^{+}$386.1727, found 386.1716.

$N$-acetyl- $N$-(3-oxo-1,3-diphenylpropyl)-2-phenylacetamide (23):<smiles>CC(=O)N(Cc1ccccc1)C(=O)Cc1ccccc1</smiles>

The compound was prepared according to the general procedure for reaction of oxime ester and styrene with petroleum ether/ethyl acetate as eluent. Yield: 55\% (42.4 mg, white solid). ${ }^{1} \mathrm{H}$ NMR $\left(400 \mathrm{MHz}, \mathrm{CDCl}_{3}\right) \delta 7.99(\mathrm{~d}, J=7.6 \mathrm{~Hz}, 2 \mathrm{H}), 7.61(\mathrm{t}, J=7.2 \mathrm{~Hz}, 1 \mathrm{H}), 7.48(\mathrm{t}, J=7.5 \mathrm{~Hz}, 2 \mathrm{H}), 7.31$ $-7.19(\mathrm{~m}, 8 \mathrm{H}), 7.17-7.09(\mathrm{~d}, J=6.7 \mathrm{~Hz}, 2 \mathrm{H}), 6.06-5.79(\mathrm{~m}, 1 \mathrm{H}), 4.22-4.02(\mathrm{~m}, 3 \mathrm{H}), 3.89(\mathrm{dd}$, $J=18.2,5.6 \mathrm{~Hz}, 1 \mathrm{H}), 2.47$ (s, 3H). ${ }^{13} \mathrm{C} \mathrm{NMR}\left(101 \mathrm{MHz}, \mathrm{CDCl}_{3}\right) \delta 197.99,175.81,174.78,139.41$, 136.69, 134.39, 133.76, 129.72, 128.92, 128.76, 128.67, 128.44, 127.55, 127.18, 126.48, 56.18, 45.30, 41.85, 26.57. HRMS (ESI): calcd for $\mathrm{C}_{25} \mathrm{H}_{23} \mathrm{NNaO}_{3}[\mathrm{M}+\mathrm{Na}]^{+} 408.1570$, found 408.1558 .

$N$-acetyl- $N$-(3-oxo-1,3-diphenylpropyl)benzamide (24):

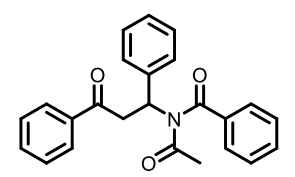

The compound was prepared according to the general procedure for reaction of oxime ester and styrene with petroleum ether/ethyl acetate as eluent. Yield: $54 \%$ (40.1 mg, colorless oil). ${ }^{1} \mathrm{H}$ NMR $\left(400 \mathrm{MHz}, \mathrm{CDCl}_{3}\right) \delta 7.92(\mathrm{~d}, J=7.6 \mathrm{~Hz}, 2 \mathrm{H}), 7.53-7.18(\mathrm{~m}, 13 \mathrm{H}), 6.20(\mathrm{dd}, J=8.7,5.3 \mathrm{~Hz}$, $1 \mathrm{H}), 4.39(\mathrm{dd}, J=17.9,9.2 \mathrm{~Hz}, 1 \mathrm{H}), 3.71(\mathrm{dd}, J=17.9,5.1 \mathrm{~Hz}, 1 \mathrm{H}), 1.80(\mathrm{~s}, 3 \mathrm{H}) .{ }^{13} \mathrm{C}$ NMR $(101$ $\left.\mathrm{MHz}, \mathrm{CDCl}_{3}\right) \delta 197.62,174.60,173.84,139.46,136.69,136.66,133.36,132.71,128.92,128.82$, 
128.67, 128.50, 128.17, 127.76, 127.65, 56.43, 41.22, 27.66. HRMS (ESI): calcd for

$\mathrm{C}_{24} \mathrm{H}_{21} \mathrm{NNaO}_{3}[\mathrm{M}+\mathrm{Na}]^{+}$394.1414, found 394.1401

$N$-acetyl- $N$-(3-oxo-1,3-diphenylpropyl)isonicotinamide (25):

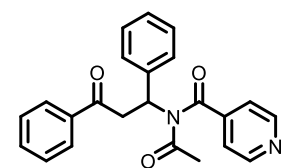

The compound was prepared according to the general procedure for reaction of oxime ester and styrene with petroleum ether/ethyl acetate as eluent. Yield: $47 \%$ (35.0 mg, pale yellow oil). ${ }^{1} \mathrm{H}$ NMR $\left(400 \mathrm{MHz}, \mathrm{CDCl}_{3}\right) \delta 8.69(\mathrm{~d}, J=4.7 \mathrm{~Hz}, 2 \mathrm{H}), 7.98(\mathrm{~d}, J=7.7 \mathrm{~Hz}, 2 \mathrm{H}), 7.58(\mathrm{t}, J=7.4 \mathrm{~Hz}, 1 \mathrm{H})$, $7.50-7.43(\mathrm{~m}, 4 \mathrm{H}), 7.41(\mathrm{~d}, J=4.7 \mathrm{~Hz}, 2 \mathrm{H}), 7.37(\mathrm{t}, J=7.4 \mathrm{~Hz}, 2 \mathrm{H}), 7.32-7.28(\mathrm{~m}, 1 \mathrm{H}), 6.06$ $(\mathrm{dd}, J=9.8,4.2 \mathrm{~Hz}, 1 \mathrm{H}), 4.55(\mathrm{dd}, J=18.2,9.9 \mathrm{~Hz}, 1 \mathrm{H}), 3.67(\mathrm{dd}, J=18.2,4.3 \mathrm{~Hz}, 1 \mathrm{H}), 2.12(\mathrm{~s}$, 3H). ${ }^{13} \mathrm{C} \mathrm{NMR}\left(101 \mathrm{MHz}, \mathrm{CDCl}_{3}\right) \delta 197.69,173.94,172.87,150.60,143.79,138.94,136.38,133.65$, 128.77, 128.14, 127.93, 127.29, 121.63, 57.11, 41.11, 27.14. HRMS (ESI): calcd for $\mathrm{C}_{23} \mathrm{H}_{21} \mathrm{~N}_{2} \mathrm{O}_{3}$ $[\mathrm{M}+\mathrm{H}]^{+}$373.1547, found 373.1547 .

$N$-acetyl- $N$-(3-oxo-1,3-diphenylpropyl)-2-naphthamide (26):

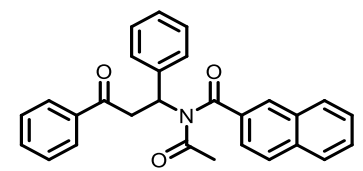

The compound was prepared according to the general procedure for reaction of oxime ester and styrene with petroleum ether/ethyl acetate as eluent. Yield: $74 \%$ (63.3 mg, white solid). ${ }^{1} \mathrm{H}$ NMR $\left(400 \mathrm{MHz}, \mathrm{CDCl}_{3}\right) \delta 8.24(\mathrm{~d}, J=8.1 \mathrm{~Hz}, 1 \mathrm{H}), 8.03(\mathrm{~d}, J=7.6 \mathrm{~Hz}, 2 \mathrm{H}), 7.92(\mathrm{~d}, J=7.9 \mathrm{~Hz}, 1 \mathrm{H})$, $7.85(\mathrm{~d}, J=7.8 \mathrm{~Hz}, 1 \mathrm{H}), 7.61-7.44(\mathrm{~m}, 7 \mathrm{H}), 7.42-7.32(\mathrm{~m}, 4 \mathrm{H}), 7.27(\mathrm{t}, J=7.6 \mathrm{~Hz}, 1 \mathrm{H}), 6.39$ $(\mathrm{dd}, J=8.7,5.4 \mathrm{~Hz}, 1 \mathrm{H}), 4.56(\mathrm{dd}, J=17.8,9.2 \mathrm{~Hz}, 1 \mathrm{H}), 3.84(\mathrm{dd}, J=17.8,5.0 \mathrm{~Hz}, 1 \mathrm{H}), 1.78(\mathrm{~s}$, $3 \mathrm{H}) .{ }^{13} \mathrm{C} \mathrm{NMR}\left(101 \mathrm{MHz}, \mathrm{CDCl}_{3}\right) \delta$ 197.80, 174.42, 173.54, 139.53, 136.78, 134.44, 133.83, 133.39, 132.21, 130.42, 128.72, 128.58, 128.53, 128.22, 128.03, 127.74, 127.04, 126.88, 125.01, 124.61, 56.11, 41.14, 27.66. HRMS (ESI): calcd for $\mathrm{C}_{28} \mathrm{H}_{23} \mathrm{NNaO}_{3}[\mathrm{M}+\mathrm{Na}]^{+} 444.1570$, found 444.1555.

\section{$N$-acetyl- $N$-(3-oxo-3-phenyl-1-(o-tolyl)propyl)acetamide (27):}<smiles></smiles>

The compound was prepared according to the general procedure for reaction of oxime ester and styrene with petroleum ether/ethyl acetate as eluent. Yield: 53\% (34.2 mg, pale yellow oil). ${ }^{1} \mathrm{H}$ NMR $\left(400 \mathrm{MHz}, \mathrm{CDCl}_{3}\right) \delta 7.97(\mathrm{~d}, J=7.8 \mathrm{~Hz}, 2 \mathrm{H}), 7.58(\mathrm{t}, J=7.3 \mathrm{~Hz}, 1 \mathrm{H}), 7.46(\mathrm{t}, J=7.6 \mathrm{~Hz}, 2 \mathrm{H}), 7.38$ $(\mathrm{d}, J=7.2 \mathrm{~Hz}, 1 \mathrm{H}), 7.22-7.12(\mathrm{~m}, 3 \mathrm{H}), 6.07-5.93(\mathrm{~m}, 1 \mathrm{H}), 4.14(\mathrm{dd}, J=18.0,8.0 \mathrm{~Hz}, 1 \mathrm{H}), 3.70$ (dd, $J=18.0,5.9 \mathrm{~Hz}, 1 \mathrm{H}), 2.34$ (s, 3H), 2.32 (s, 6H). ${ }^{13} \mathrm{C} \mathrm{NMR}\left(101 \mathrm{MHz}, \mathrm{CDCl}_{3}\right) \delta 197.51,174.81$, 
137.19, 136.34, 134.86, 133.58, 130.98, 128.77, 128.18, 127.84, 127.77, 126.45, 54.36, 40.84, 26.66, 19.42. HRMS (ESI): calcd for $\mathrm{C}_{20} \mathrm{H}_{21} \mathrm{NNaO}_{3}[\mathrm{M}+\mathrm{Na}]^{+} 346.1414$, found 346.1407 .

$N$-acetyl- $N$-(3-oxo-3-phenyl-1-(m-tolyl)propyl)acetamide (28):<smiles>CC(=O)N(CC(=O)c1ccccc1)C(C)=O</smiles>

The compound was prepared according to the general procedure for reaction of oxime ester and styrene with petroleum ether/ethyl acetate as eluent. Yield: $66 \%$ (42.6 mg, white solid). ${ }^{1} \mathrm{H}$ NMR $\left(400 \mathrm{MHz}, \mathrm{CDCl}_{3}\right) \delta 8.00(\mathrm{~d}, J=7.7 \mathrm{~Hz}, 2 \mathrm{H}), 7.59(\mathrm{t}, J=7.1 \mathrm{~Hz}, 1 \mathrm{H}), 7.47(\mathrm{t}, J=7.6 \mathrm{~Hz}, 2 \mathrm{H}), 7.22$ $(\mathrm{t}, J=7.9 \mathrm{~Hz}, 1 \mathrm{H}), 7.12-7.04(\mathrm{~m}, 3 \mathrm{H}), 5.98-5.72(\mathrm{~m}, 1 \mathrm{H}), 4.27(\mathrm{dd}, J=18.1,7.9 \mathrm{~Hz}, 1 \mathrm{H}), 3.81$ (dd, $J=18.1,5.1 \mathrm{~Hz}, 1 \mathrm{H}), 2.45$ (s, 6H), 2.34 (s, 3H). $\left.{ }^{13} \mathrm{C} \mathrm{NMR} \mathrm{(101} \mathrm{MHz,} \mathrm{CDCl}_{3}\right) \delta 198.09,174.49$, 139.39, 138.34, 136.49, 133.63, 128.78, 128.58, 128.26, 128.21, 126.78, 123.16, 55.97, 41.62, 26.87, 21.62. HRMS (ESI): calcd for $\mathrm{C}_{20} \mathrm{H}_{21} \mathrm{NNaO}_{3}[\mathrm{M}+\mathrm{Na}]^{+} 346.1414$, found 346.1406 .

$N$-acetyl- $N$-(3-oxo-3-phenyl-1-(p-tolyl)propyl)acetamide (29):<smiles>CC(=O)N(C(C)=O)C(C(=O)c1ccccc1)C(=O)c1ccc(C)cc1</smiles>

The compound was prepared according to the general procedure for reaction of oxime ester and styrene with petroleum ether/ethyl acetate as eluent. Yield: $64 \%\left(41.3 \mathrm{mg}\right.$, white solid). ${ }^{1} \mathrm{H}$ NMR $\left(400 \mathrm{MHz}, \mathrm{CDCl}_{3}\right) \delta 7.92(\mathrm{~d}, J=7.8 \mathrm{~Hz}, 2 \mathrm{H}), 7.51(\mathrm{t}, J=7.3 \mathrm{~Hz}, 1 \mathrm{H}), 7.40(\mathrm{t}, J=7.6 \mathrm{~Hz}, 2 \mathrm{H}), 7.11$ $(\mathrm{d}, \mathrm{J}=8.0 \mathrm{~Hz}, 2 \mathrm{H}), 7.06(\mathrm{~d}, \mathrm{~J}=8.0 \mathrm{~Hz}, 2 \mathrm{H}), 5.82-5.71(\mathrm{~m}, 1 \mathrm{H}), 4.18(\mathrm{dd}, J=18.1,7.8 \mathrm{~Hz}, 1 \mathrm{H})$, $3.74(\mathrm{dd}, J=18.1,5.3 \mathrm{~Hz}, 1 \mathrm{H}), 2.37(\mathrm{~s}, 6 \mathrm{H}), 2.25(\mathrm{~s}, 3 \mathrm{H}) .{ }^{13} \mathrm{C} \mathrm{NMR}\left(101 \mathrm{MHz}, \mathrm{CDCl}_{3}\right) \delta$ 198.07, 174.45, 137.11, 136.48, 136.36, 133.60, 129.34, 128.76, 128.24, 126.08, 55.83, 41.58, 26.87, 20.98. HRMS (ESI): calcd for $\mathrm{C}_{20} \mathrm{H}_{21} \mathrm{NNaO}_{3}[\mathrm{M}+\mathrm{Na}]^{+} 346.1414$, found 346.1405 .

$N$-acetyl- $N$-(1-(2,5-dimethylphenyl)-3-oxo-3-phenylpropyl)acetamide (30):<smiles></smiles>

The compound was prepared according to the general procedure for reaction of oxime ester and styrene with petroleum ether/ethyl acetate as eluent. Yield: 54\% (36.4 mg, white solid). ${ }^{1} \mathrm{H}$ NMR $\left(400 \mathrm{MHz}, \mathrm{CDCl}_{3}\right) \delta 7.97$ (d, $\left.J=7.4 \mathrm{~Hz}, 2 \mathrm{H}\right), 7.58(\mathrm{t}, J=7.0 \mathrm{~Hz}, 1 \mathrm{H}), 7.47$ (t, $\left.J=7.2 \mathrm{~Hz}, 2 \mathrm{H}\right), 7.18$ (s, 1H), $7.05(\mathrm{~d}, J=7.5 \mathrm{~Hz}, 1 \mathrm{H}), 7.00(\mathrm{~d}, J=7.5 \mathrm{~Hz}, 1 \mathrm{H}), 5.98(\mathrm{t}, J=6.0 \mathrm{~Hz}, 1 \mathrm{H}), 4.21(\mathrm{dd}, J=$ 18.0, $8.4 \mathrm{~Hz}, 1 \mathrm{H}), 3.58(\mathrm{dd}, J=18.1,4.5 \mathrm{~Hz}, 1 \mathrm{H}), 2.33(\mathrm{~s}, 6 \mathrm{H}), 2.29$ (s, 3H), $2.28(\mathrm{~s}, 3 \mathrm{H}) .{ }^{13} \mathrm{C} \mathrm{NMR}$ $\left(101 \mathrm{MHz}, \mathrm{CDCl}_{3}\right) \delta 197.66,175.00,137.19,136.35,136.03,133.56,131.45,130.86,128.76$, $128.57,128.34,128.18,54.58,40.89,26.64,21.26,18.93$. HRMS (ESI): calcd for $\mathrm{C}_{21} \mathrm{H}_{23} \mathrm{NNaO}_{3}$ $[\mathrm{M}+\mathrm{Na}]^{+} 360.1570$, found 360.1559 . 
<smiles>CC(=O)N(Cc1ccc(C(C)(C)C)cc1)C(C)=O</smiles>

The compound was prepared according to the general procedure for reaction of oxime ester and styrene with petroleum ether/ethyl acetate as eluent. Yield: 55\% (40.2 mg, yellow soild). ${ }^{1} \mathrm{H}$ NMR $\left(400 \mathrm{MHz}, \mathrm{CDCl}_{3}\right) \delta 8.00(\mathrm{~d}, J=7.7 \mathrm{~Hz}, 2 \mathrm{H}), 7.60(\mathrm{t}, J=7.3 \mathrm{~Hz}, 1 \mathrm{H}), 7.48(\mathrm{t}, J=7.6 \mathrm{~Hz}, 2 \mathrm{H}), 7.35$ $(\mathrm{d}, J=8.3 \mathrm{~Hz}, 2 \mathrm{H}), 7.22(\mathrm{~d}, J=8.3 \mathrm{~Hz}, 2 \mathrm{H}), 5.85(\mathrm{dd}, J=7.2,5.8 \mathrm{~Hz}, 1 \mathrm{H}), 4.28(\mathrm{dd}, J=18.1,7.9$ $\mathrm{Hz}, 1 \mathrm{H}), 3.82(\mathrm{dd}, J=18.1,5.2 \mathrm{~Hz}, 1 \mathrm{H}), 2.46(\mathrm{~s}, 6 \mathrm{H}), 1.30$ (s, 9H). ${ }^{13} \mathrm{C} \mathrm{NMR}\left(101 \mathrm{MHz}, \mathrm{CDCl}_{3}\right) \delta$ 199.48, 175.84, 151.68, 138.01, 137.77, 134.89, 130.11, 129.61, 127.25, 126.95, 57.28, 43.18, 35.81, 32.65, 28.13, 28.12. HRMS (ESI): calcd for $\mathrm{C}_{23} \mathrm{H}_{27} \mathrm{NNaO}_{3}[\mathrm{M}+\mathrm{Na}]^{+} 388.1883$, found 388.1873.

$N$-acetyl- $N$-(1-(2-fluorophenyl)-3-oxo-3-phenylpropyl)acetamide (32):<smiles></smiles>

The compound was prepared according to the general procedure for reaction of oxime ester and styrene with petroleum ether/ethyl acetate as eluent. Yield: 63\% (41.2 mg, white solid). ${ }^{1} \mathrm{H}$ NMR $\left(400 \mathrm{MHz}, \mathrm{CDCl}_{3}\right) \delta 7.99(\mathrm{~d}, J=7.8 \mathrm{~Hz}, 2 \mathrm{H}), 7.60(\mathrm{t}, J=7.2 \mathrm{~Hz}, 1 \mathrm{H}), 7.52-7.44(\mathrm{~m}, 3 \mathrm{H}), 7.37-$ $7.18(\mathrm{~m}, 2 \mathrm{H}), 7.14-7.02(\mathrm{~m}, 2 \mathrm{H}), 6.07-5.88(\mathrm{~m}, 1 \mathrm{H}), 4.22(\mathrm{dd}, J=18.1,7.7 \mathrm{~Hz}, 1 \mathrm{H}), 3.93(\mathrm{dd}, J$ $=18.1,5.7 \mathrm{~Hz}, 1 \mathrm{H}), 2.46(\mathrm{~s}, 6 \mathrm{H}) .{ }^{13} \mathrm{C} \mathrm{NMR}\left(101 \mathrm{MHz}, \mathrm{CDCl}_{3}\right) \delta 197.45,174.62,160.40(\mathrm{~d}, J=$ $244.0 \mathrm{~Hz}), 136.37,133.68,129.72(\mathrm{~d}, J=3.5 \mathrm{~Hz}), 129.48(\mathrm{~d}, J=8.7 \mathrm{~Hz}), 128.79,128.19,125.68$ (d, $J=12.5 \mathrm{~Hz}), 124.00$ (d, $J=3.3 \mathrm{~Hz}), 115.54$ (d, $J=22.4 \mathrm{~Hz}), 51.31$ (d, $J=1.6 \mathrm{~Hz}), 40.09,26.55$, 26.53. ${ }^{19} \mathrm{~F}$ NMR (377 MHz, CDCl3) $\delta-116.10$. HRMS (ESI): calcd for $\mathrm{C}_{19} \mathrm{H}_{18} \mathrm{FNNaO}_{3}[\mathrm{M}+\mathrm{Na}]^{+}$ 350.1163 , found 350.1152 .

$N$-acetyl- $N$-(1-(4-fluorophenyl)-3-oxo-3-phenylpropyl)acetamide (33):<smiles>CC(=O)N(C(C)=O)C(C(=O)c1ccccc1)C(=O)c1ccc(F)cc1</smiles>

The compound was prepared according to the general procedure for reaction of oxime ester and styrene with petroleum ether/ethyl acetate as eluent. Yield: $89 \%$ (58.2 mg, white solid). ${ }^{1} \mathrm{H}$ NMR $\left(400 \mathrm{MHz}, \mathrm{CDCl}_{3}\right) \delta 7.99$ (d, $\left.J=7.6 \mathrm{~Hz}, 2 \mathrm{H}\right), 7.60(\mathrm{t}, J=7.2 \mathrm{~Hz}, 1 \mathrm{H}), 7.48(\mathrm{t}, J=7.5 \mathrm{~Hz}, 2 \mathrm{H}), 7.33$ $-7.27(\mathrm{~m}, 2 \mathrm{H}), 7.05-6.99(\mathrm{~m}, 2 \mathrm{H}), 5.91-5.77(\mathrm{~m}, 1 \mathrm{H}), 4.24(\mathrm{dd}, J=18.2,7.6 \mathrm{~Hz}, 1 \mathrm{H}), 3.83(\mathrm{dd}$, $J=18.2,5.3 \mathrm{~Hz}, 1 \mathrm{H}), 2.45$ (s, 6H). ${ }^{13} \mathrm{C} \mathrm{NMR}\left(101 \mathrm{MHz}, \mathrm{CDCl}_{3}\right) \delta 197.77,174.31,161.94(\mathrm{~d}, J=$ $244.9 \mathrm{~Hz}), 136.31,135.15$ (d, $J=3.3 \mathrm{~Hz}), 133.74,128.81,128.22,128.13$ (d, $J=8.0 \mathrm{~Hz}), 115.49$ (d, $J=21.4 \mathrm{~Hz}), 55.48,41.59,26.88 .{ }^{19} \mathrm{~F}$ NMR $(377 \mathrm{MHz}, \mathrm{CDCl} 3) \delta-115.01$. HRMS (ESI): calcd for $\mathrm{C}_{19} \mathrm{H}_{18} \mathrm{FNNaO}_{3}[\mathrm{M}+\mathrm{Na}]^{+} 350.1163$, found 350.1151 . 
<smiles>CC(=O)N(C(C)=O)C(C(=O)c1ccccc1)C(=O)c1ccc(Cl)cc1</smiles>

The compound was prepared according to the general procedure for reaction of oxime ester and styrene with petroleum ether/ethyl acetate as eluent. Yield: $71 \%$ (48.7 mg, pale yellow oil). ${ }^{1} \mathrm{H}$ NMR $\left(400 \mathrm{MHz}, \mathrm{CDCl}_{3}\right) \delta 7.99$ (d, $\left.J=7.9 \mathrm{~Hz}, 2 \mathrm{H}\right), 7.60(\mathrm{t}, J=7.3 \mathrm{~Hz}, 1 \mathrm{H}), 7.48(\mathrm{t}, J=7.5 \mathrm{~Hz}, 2 \mathrm{H}), 7.30$ $(\mathrm{d}, J=7.9 \mathrm{~Hz}, 2 \mathrm{H}), 7.24(\mathrm{~d}, J=8.2 \mathrm{~Hz}, 2 \mathrm{H}), 5.90-5.78(\mathrm{~m}, 1 \mathrm{H}), 4.21(\mathrm{dd}, J=18.2,7.5 \mathrm{~Hz}, 1 \mathrm{H})$, $3.83(\mathrm{dd}, J=18.2,5.4 \mathrm{~Hz}, 1 \mathrm{H}), 2.45(\mathrm{~s}, 6 \mathrm{H}) .{ }^{13} \mathrm{C} \mathrm{NMR}\left(101 \mathrm{MHz}, \mathrm{CDCl}_{3}\right) \delta 197.69,174.23,137.97$, 136.30, 133.78, 133.29, 128.83, 128.79, 128.24, 127.73, 55.46, 41.46, 26.86. HRMS (ESI): calcd for $\mathrm{C}_{19} \mathrm{H}_{18} \mathrm{ClNNaO}_{3}[\mathrm{M}+\mathrm{Na}]^{+} 366.0867$, found 366.0858 .

$N$-acetyl- $N$-(1-(4-bromophenyl)-3-oxo-3-phenylpropyl)acetamide (35):<smiles>CC(=O)N(C(C)=O)C(C(=O)c1ccccc1)C(=O)c1ccccc1</smiles>

The compound was prepared according to the general procedure for reaction of oxime ester and styrene with petroleum ether/ethyl acetate as eluent. Yield: $90 \%$ (69.8 mg, yellow solid). ${ }^{1} \mathrm{H}$ NMR $\left(400 \mathrm{MHz}, \mathrm{CDCl}_{3}\right) \delta 7.99(\mathrm{~d}, J=7.7 \mathrm{~Hz}, 2 \mathrm{H}), 7.61(\mathrm{t}, J=7.2 \mathrm{~Hz}, 1 \mathrm{H}), 7.52-7.44(\mathrm{~m}, 4 \mathrm{H}), 7.18(\mathrm{~d}$, $J=7.9 \mathrm{~Hz}, 2 \mathrm{H}), 5.88-5.76(\mathrm{~m}, 1 \mathrm{H}), 4.20(\mathrm{dd}, J=18.2,7.4 \mathrm{~Hz}, 1 \mathrm{H}), 3.84(\mathrm{dd}, J=18.2,5.2 \mathrm{~Hz}$,

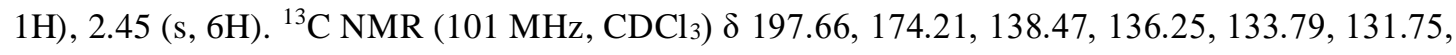
$128.83,128.23,128.02,121.38,55.49,41.42,26.88$. HRMS (ESI): calcd for $\mathrm{C}_{19} \mathrm{H}_{18} \mathrm{BrNNaO}_{3}$ $[\mathrm{M}+\mathrm{Na}]^{+}$410.0362, 412.0342, found 410.0351, 412.0330 .

$N$-(1-([1,1'-biphenyl]-4-yl)-3-oxo-3-phenylpropyl)- $N$-acetylacetamide (36):<smiles>CC(=O)N(C(C)=O)C(C(=O)c1ccccc1)c1ccc(-c2ccccc2)cc1</smiles>

The compound was prepared according to the general procedure for reaction of oxime ester and styrene with petroleum ether/ethyl acetate as eluent. Yield: 62\% (47.7 mg, pale yellow oil). ${ }^{1} \mathrm{H}$ NMR $\left(400 \mathrm{MHz}, \mathrm{CDCl}_{3}\right) \delta 7.98-7.88(\mathrm{~m}, 2 \mathrm{H}), 7.82-7.69(\mathrm{~m}, 3 \mathrm{H}), 7.66-7.60(\mathrm{~m}, 1 \mathrm{H}), 7.57-7.50(\mathrm{~m}$, 2H), $7.47-7.35(\mathrm{~m}, 4 \mathrm{H}), 7.33-7.28(\mathrm{~m}, 1 \mathrm{H}), 7.19(\mathrm{~s}, 1 \mathrm{H}), 6.38(\mathrm{dd}, J=8.5,5.2 \mathrm{~Hz}, 1 \mathrm{H}), 4.52(\mathrm{dd}$, $J=17.9,9.3 \mathrm{~Hz}, 1 \mathrm{H}), 3.82(\mathrm{dd}, J=17.9,5.0 \mathrm{~Hz}, 1 \mathrm{H}), 1.80(\mathrm{~s}, 3 \mathrm{H}), 1.51(\mathrm{~s}, 3 \mathrm{H}) .{ }^{13} \mathrm{C}$ NMR $(101$ $\left.\mathrm{MHz}, \mathrm{CDCl}_{3}\right) \delta 197.99,174.44,140.50,140.35,138.39,136.43,133.70,128.80,128.27,127.40$, 127.07, 126.58, 55.81, 41.65, 26.91. HRMS (ESI): calcd for $\mathrm{C}_{25} \mathrm{H}_{23} \mathrm{NNaO}_{3}[\mathrm{M}+\mathrm{Na}]^{+} 408.1570$, found 408.1564 .

$N$-acetyl- $N$-(1-(naphthalen-2-yl)-3-oxo-3-phenylpropyl)acetamide(37): 


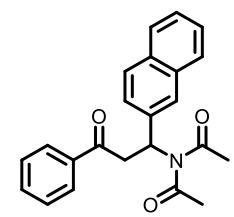

The compound was prepared according to the general procedure for reaction of oxime ester and styrene with petroleum ether/ethyl acetate as eluent. Yield: $69 \%$ (49.6 mg, colorless oil). ${ }^{1} \mathrm{H}$ NMR $\left(400 \mathrm{MHz} \mathrm{CDCl}_{3}\right) \delta 8.04(\mathrm{~d}, J=7.6 \mathrm{~Hz}, 2 \mathrm{H}), 7.86-7.78(\mathrm{~m}, 3 \mathrm{H}), 7.74(\mathrm{~s}, 1 \mathrm{H}), 7.62(\mathrm{t}, J=7.3 \mathrm{~Hz}$, 1H), $7.53-7.42(\mathrm{~m}, 5 \mathrm{H}), 6.12-5.98(\mathrm{~m}, 1 \mathrm{H}), 4.38(\mathrm{dd}, J=18.1,7.8 \mathrm{~Hz}, 1 \mathrm{H}), 3.97(\mathrm{dd}, J=18.1$, $5.1 \mathrm{~Hz}, 1 \mathrm{H}), 2.49$ (s, 6H). ${ }^{13} \mathrm{C} \mathrm{NMR}\left(101 \mathrm{MHz}, \mathrm{CDCl}_{3}\right) \delta 198.00,174.51,136.76,136.45,133.70$, $133.21,132.58,128.81,128.53,128.28,128.04,127.56,126.40,126.16,125.15,124.22,56.16$, 41.60, 26.93. HRMS (ESI): calcd for $\mathrm{C}_{23} \mathrm{H}_{21} \mathrm{NNaO}_{3}[\mathrm{M}+\mathrm{Na}]^{+} 382.1414$, found 382.1407.

\section{$N$-acetyl- $N$-(3-oxo-1,3-diphenylpropyl)butyramide (38):}

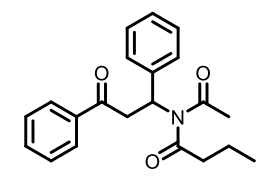

The compound was prepared according to the general procedure for reaction of oxime ester and styrene with petroleum ether/ethyl acetate as eluent. Yield: $51 \%$ (34.4 mg, white solid). ${ }^{1} \mathrm{H}$ NMR $\left(400 \mathrm{MHz} \mathrm{CDCl}_{3}\right) \delta 8.00(\mathrm{~d}, J=7.7 \mathrm{~Hz}, 2 \mathrm{H}), 7.59(\mathrm{t}, J=7.3 \mathrm{~Hz}, 1 \mathrm{H}), 7.48(\mathrm{t}, J=7.5 \mathrm{~Hz}, 2 \mathrm{H}), 7.34$ $-7.25(\mathrm{~m}, 5 \mathrm{H}), 6.00-5.77(\mathrm{~m}, 1 \mathrm{H}), 4.20(\mathrm{dd}, J=18.1,7.4 \mathrm{~Hz}, 1 \mathrm{H}), 3.89(\mathrm{dd}, J=18.1,5.5 \mathrm{~Hz}, 1 \mathrm{H})$, $2.81(\mathrm{dt}, J=16.5,7.2 \mathrm{~Hz}, 1 \mathrm{H}), 2.65(\mathrm{dt}, J=16.0,7.9 \mathrm{~Hz}, 1 \mathrm{H}), 2.44(\mathrm{~s}, 3 \mathrm{H}), 1.72-1.53(\mathrm{~m}, 2 \mathrm{H})$, $0.89(\mathrm{t}, J=7.4 \mathrm{~Hz}, 3 \mathrm{H}) .{ }^{13} \mathrm{C} \mathrm{NMR}\left(101 \mathrm{MHz}, \mathrm{CDCl}_{3}\right) \delta 197.98,177.45,174.43,139.54,136.44$, 133.64, 128.77, 128.65, 128.26, 127.32, 126.07, 55.44, 41.85, 40.17, 26.89, 18.45, 13.63. HRMS (ESI): calcd for $\mathrm{C}_{21} \mathrm{H}_{23} \mathrm{NNaO}_{3}[\mathrm{M}+\mathrm{Na}]^{+} 360.1570$, found 360.1554 .

$N$-acetyl- $N$-(3-oxo-1,3-diphenylpropyl)pentanamide (39):

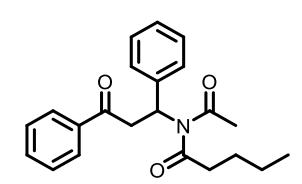

The compound was prepared according to the general procedure for reaction of oxime ester and styrene with petroleum ether/ethyl acetate as eluent. Yield: $66 \%$ (46.3 mg, colorless oil). ${ }^{1} \mathrm{H}$ NMR $\left(400 \mathrm{MHz}, \mathrm{CDCl}_{3}\right) \delta 7.94(\mathrm{~d}, J=7.7 \mathrm{~Hz}, 2 \mathrm{H}), 7.53(\mathrm{t}, J=7.2 \mathrm{~Hz}, 1 \mathrm{H}), 7.42(\mathrm{t}, J=7.5 \mathrm{~Hz}, 2 \mathrm{H}), 7.29$ $-7.19(\mathrm{~m}, 5 \mathrm{H}), 5.97-5.74(\mathrm{t}, J=6.2 \mathrm{~Hz}, 1 \mathrm{H}), 4.12(\mathrm{dd}, J=18.1,7.4 \mathrm{~Hz}, 1 \mathrm{H}), 3.82(\mathrm{dd}, J=18.0$, $5.5 \mathrm{~Hz}, 1 \mathrm{H}), 2.75(\mathrm{dt}, J=16.3,7.4 \mathrm{~Hz}, 1 \mathrm{H}), 2.59(\mathrm{dt}, J=16.3,7.4 \mathrm{~Hz}, 1 \mathrm{H}), 2.37(\mathrm{~s}, 3 \mathrm{H}), 1.52-1.49$ $(\mathrm{m}, 2 \mathrm{H}), 1.24-1.18(\mathrm{~m}, 6.8 \mathrm{~Hz}, 2 \mathrm{H}), 0.79(\mathrm{t}, J=7.3 \mathrm{~Hz}, 3 \mathrm{H}) .{ }^{13} \mathrm{C} \mathrm{NMR}\left(101 \mathrm{MHz}, \mathrm{CDCl}_{3}\right) \delta$ 197.97, 177.66, 174.44, 139.55, 136.45, 133.62, 128.76, 128.64, 128.25, 127.32, 126.08, 55.48, 41.85, 38.03, 27.11, 26.84, 22.20, 13.81. HRMS (ESI): calcd for $\mathrm{C}_{22} \mathrm{H}_{25} \mathrm{NNaO}_{3}[\mathrm{M}+\mathrm{Na}]^{+} 374.1727$, found 374.1712 .

\section{$N$-acetyl- $N$-(3-oxo-1,3-diphenylpropyl)isobutyramide (40):}




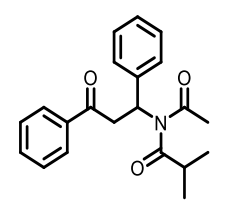

The compound was prepared according to the general procedure for reaction of oxime ester and styrene with petroleum ether/ethyl acetate as eluent. Yield: 52\% (35.1 mg, white solid). ${ }^{1} \mathrm{H}$ NMR $\left(400 \mathrm{MHz} \mathrm{CDCl}_{3}\right) \delta 8.01(\mathrm{~d}, J=7.7 \mathrm{~Hz}, 2 \mathrm{H}), 7.59(\mathrm{t}, J=7.3 \mathrm{~Hz}, 1 \mathrm{H}), 7.48(\mathrm{t}, J=7.6 \mathrm{~Hz}, 2 \mathrm{H}), 7.33$ $-7.24(\mathrm{~m}, 5 \mathrm{H}), 6.02-5.87(\mathrm{~m}, 1 \mathrm{H}), 4.13(\mathrm{dd}, J=18.1,7.2 \mathrm{~Hz}, 1 \mathrm{H}), 3.91(\mathrm{dd}, J=18.1,5.7 \mathrm{~Hz}, 1 \mathrm{H})$, $3.26-3.12(\mathrm{~m}, 1 \mathrm{H}), 2.44(\mathrm{~s}, 3 \mathrm{H}), 1.06(\mathrm{dd}, J=6.9 \mathrm{~Hz}, 6 \mathrm{H}) .{ }^{13} \mathrm{C} \mathrm{NMR}\left(101 \mathrm{MHz}, \mathrm{CDCl}_{3}\right) \delta 197.76$, $182.82,174.52,139.51,136.44,133.62,128.77,128.67,128.62,128.26,127.38,126.33,55.46$, 41.96, 35.96, 26.28, 19.65, 19.48. HRMS (ESI): calcd for $\mathrm{C}_{21} \mathrm{H}_{23} \mathrm{NNaO}_{3}[\mathrm{M}+\mathrm{Na}]^{+} 360.1570$, found 360.1556 .

$N$-acetyl- $N$-(3-oxo-1,3-diphenylpropyl)cyclopropanecarboxamide (20'):<smiles>CC(=O)N(C(=O)C(=O)c1ccccc1)C(CC(=O)c1ccccc1)c1ccccc1</smiles>

The compound was prepared according to the general procedure for reaction of oxime ester and styrene with petroleum ether/ethyl acetate as eluent. Yield: 81\% (54.3 mg, white solid). ${ }^{1} \mathrm{H}$ NMR $\left(400 \mathrm{MHz}, \mathrm{CDCl}_{3}\right) \delta 7.93(\mathrm{~d}, J=7.6 \mathrm{~Hz}, 2 \mathrm{H}), 7.52(\mathrm{t}, J=7.2 \mathrm{~Hz}, 1 \mathrm{H}), 7.41(\mathrm{t}, J=7.4 \mathrm{~Hz}, 2 \mathrm{H}), 7.31$ $-7.15(\mathrm{~m}, 5 \mathrm{H}), 6.26-5.95(\mathrm{~m}, 1 \mathrm{H}), 4.14(\mathrm{dd}, J=17.9,7.6 \mathrm{~Hz}, 1 \mathrm{H}), 3.81(\mathrm{dd}, J=18.0,5.6 \mathrm{~Hz}, 1 \mathrm{H})$, $2.32(\mathrm{~s}, 3 \mathrm{H}), 2.08-1.97(\mathrm{~m}, 1 \mathrm{H}), 1.15-0.97(\mathrm{~m}, 2 \mathrm{H}), 0.93-0.80(\mathrm{~m}, 2 \mathrm{H}) .{ }^{13} \mathrm{C}$ NMR $(101 \mathrm{MHz}$, $\left.\mathrm{CDCl}_{3}\right) \delta 197.84,179.02,173.63,139.68,136.58,133.53,128.74,128.58,128.25,127.34,126.53$, 55.45, 41.75, 26.61, 17.11, 11.16, 11.04. HRMS (ESI): calcd for $\mathrm{C}_{21} \mathrm{H}_{21} \mathrm{NNaO}_{3}[\mathrm{M}+\mathrm{Na}]^{+}$ 358.1414 , found 358.1400 .

\section{$N$-acetyl- $N$-(3-oxo-1,3-diphenylpropyl)acetamide-d3 (41):}

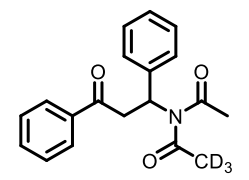

The compound was prepared according to the general procedure for reaction of oxime ester and styrene with petroleum ether/ethyl acetate as eluent. ${ }^{1} \mathrm{H}$ NMR Yield: $60 \%$, white solid. ${ }^{1} \mathrm{H}$ NMR $\left(400 \mathrm{MHz}, \mathrm{CDCl}_{3}\right) \delta 8.00(\mathrm{~d}, J=7.7 \mathrm{~Hz}, 2 \mathrm{H}), 7.60(\mathrm{t}, J=7.2 \mathrm{~Hz}, 1 \mathrm{H}), 7.48(\mathrm{t}, J=7.5 \mathrm{~Hz}, 2 \mathrm{H}), 7.36$ $-7.25(\mathrm{~m}, 5 \mathrm{H}), 5.97-5.79(\mathrm{~m}, 1 \mathrm{H}), 4.26(\mathrm{dd}, J=18.1,7.8 \mathrm{~Hz}, 1 \mathrm{H}), 3.85(\mathrm{dd}, J=18.2,5.2 \mathrm{~Hz}, 1 \mathrm{H})$, $2.46(\mathrm{~s}, 3 \mathrm{H}) .{ }^{13} \mathrm{C} \mathrm{NMR}\left(101 \mathrm{MHz}, \mathrm{CDCl}_{3}\right) \delta 198.00,174.42,139.39,136.42,133.66,128.79,128.68$, 128.25, 127.40, 126.09, 55.93, 41.59, 26.86, 26.20 (h, $J=19.9 \mathrm{~Hz})$. HRMS (ESI): calcd for $\mathrm{C}_{19} \mathrm{H}_{16} \mathrm{D}_{3} \mathrm{NNaO}_{3}[\mathrm{M}+\mathrm{Na}]^{+}$335.1445, found 335.1426.

$N$-acetyl- $N$-(3-oxo-1,3-diphenylpropyl)acetamide-d 3 (41'): 
<smiles>CC(=O)C(CC(=O)c1ccccc1)(C(C)=O)N(C)C(=O)c1ccccc1</smiles>

The compound was prepared according to the general procedure for reaction of oxime ester and styrene with petroleum ether/ethyl acetate as eluent. Yield: $61 \%$ (38.1 mg, white solid). ${ }^{1} \mathrm{H}$ NMR $\left(400 \mathrm{MHz}, \mathrm{CDCl}_{3}\right) \delta 8.01(\mathrm{~d}, J=7.8 \mathrm{~Hz}, 2 \mathrm{H}), 7.59(\mathrm{t}, J=7.2 \mathrm{~Hz}, 1 \mathrm{H}), 7.48(\mathrm{t}, J=7.7 \mathrm{~Hz}, 2 \mathrm{H}), 7.37$ $-7.25(\mathrm{~m}, 5 \mathrm{H}), 5.88(\mathrm{dd}, J=7.3,5.8 \mathrm{~Hz}, 1 \mathrm{H}), 4.27(\mathrm{dd}, J=18.1,7.8 \mathrm{~Hz}, 1 \mathrm{H}), 3.85(\mathrm{dd}, J=18.1$, $5.3 \mathrm{~Hz}, 1 \mathrm{H}), 2.46(\mathrm{~s}, 3 \mathrm{H}) .{ }^{13} \mathrm{C} \mathrm{NMR}\left(101 \mathrm{MHz}, \mathrm{CDCl}_{3}\right) \delta 198.00,174.41,139.40,136.42,133.66$, 128.79, 128.68, 128.25, 127.40, 126.09, 55.92, 41.59, 26.86, 26.20 (h, $J=19.8 \mathrm{~Hz})$. HRMS (ESI): calcd for $\mathrm{C}_{19} \mathrm{H}_{16} \mathrm{D}_{3} \mathrm{NNaO}_{3}[\mathrm{M}+\mathrm{Na}]^{+} 335.1445$, found 335.1426 .

$N$-acetyl- $N$-(5-cyano-1-phenylpentyl)-4-(trifluoromethyl)benzamide (42):

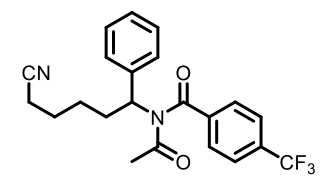

The compound was prepared according to the general procedure for reaction of oxime ester and styrene with petroleum ether/ethyl acetate as eluent. Yield: $84 \%$ (67.5 mg, colorless oil). ${ }^{1} \mathrm{H}$ NMR $\left(400 \mathrm{MHz}, \mathrm{CDCl}_{3}\right) \delta 7.69-7.63(\mathrm{~m}, 4 \mathrm{H}), 7.43(\mathrm{~d}, J=7.6 \mathrm{~Hz}, 2 \mathrm{H}), 7.31(\mathrm{t}, J=7.5 \mathrm{~Hz}, 2 \mathrm{H}), 7.27-$ $7.25(\mathrm{~m}, 1 \mathrm{H}), 5.71(\mathrm{t}, J=7.8 \mathrm{~Hz}, 1 \mathrm{H}), 2.49-2.39(\mathrm{~m}, 1 \mathrm{H}), 2.36(\mathrm{t}, J=7.0 \mathrm{~Hz}, 2 \mathrm{H}), 2.32-2.23(\mathrm{~m}$, $1 \mathrm{H}), 1.88(\mathrm{~s}, 3 \mathrm{H}), 1.81-1.70(\mathrm{~m}, 2 \mathrm{H}), 1.59-1.51(\mathrm{~m}, 2 \mathrm{H}) .{ }^{13} \mathrm{C} \mathrm{NMR}\left(101 \mathrm{MHz}, \mathrm{CDCl}_{3}\right) \delta 173.17$, $172.88,139.83,138.88,134.28(\mathrm{q}, J=32.8 \mathrm{~Hz}), 128.95,128.54,128.12,127.97,126.01$ (q, $J=3.6$ $\mathrm{Hz}), 123.33(\mathrm{q}, J=271.1 \mathrm{~Hz}), 119.41,60.14,31.25,27.55,26.10,25.08,17.09 .{ }^{19} \mathrm{~F} \mathrm{NMR}(377 \mathrm{MHz}$, $\mathrm{CDCl}_{3}$ ) $\delta$-63.15. HRMS (ESI): calcd for $\mathrm{C}_{22} \mathrm{H}_{21} \mathrm{~F}_{3} \mathrm{~N}_{2} \mathrm{NaO}_{2}[\mathrm{M}+\mathrm{Na}]^{+} 425.1447$, found 425.1434 .

Tert-butyl 5-( $N$-acetyl-4-(trifluoromethyl)benzamido)-2-(cyanomethyl)-5-phenylpentanoate (43):

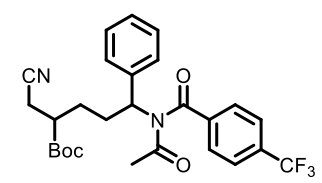

The compound was prepared according to the general procedure for reaction of oxime ester and styrene with petroleum ether/ethyl acetate as eluent. Yield: $43 \%$ (43.2 mg, white solid). ${ }^{1} \mathrm{H}$ NMR $\left(400 \mathrm{MHz}, \mathrm{CDCl}_{3}\right) \delta 7.83-7.70(\mathrm{~m}, 4 \mathrm{H}), 7.53(\mathrm{~d}, J=7.5 \mathrm{~Hz}, 2 \mathrm{H}), 7.42(\mathrm{t}, J=7.4 \mathrm{~Hz}, 2 \mathrm{H}), 7.39-$ $7.36(\mathrm{~m}, 1 \mathrm{H}), 5.87-5.76(\mathrm{~m}, 1 \mathrm{H}), 2.88-2.70(\mathrm{~m}, 2 \mathrm{H}), 2.69-2.33(\mathrm{~m}, 3 \mathrm{H}), 1.99(\mathrm{~s}, 3 \mathrm{H}), 1.96-$ $1.80(\mathrm{~m}, 2 \mathrm{H}), 1.60-1.55(\mathrm{~m}, 9 \mathrm{H}) .{ }^{13} \mathrm{C}$ NMR $\left(101 \mathrm{MHz}, \mathrm{CDCl}_{3}\right) \delta 173.12,173.09,172.85,172.75$, 171.30, 171.29, 139.76, 138.69, 138.46, 134.32 (q, $J=32.6 \mathrm{~Hz}), 128.96,128.58,128.12$, 128.07, $128.04,126.01$ (q, $J=3.6 \mathrm{~Hz}), 123.31$ (q, $J=271.0 \mathrm{~Hz}), 117.75,117.66,82.36,82.34,60.14,59.83$, 42.10, 41.76, 29.16, 28.82, 28.76, 28.70, 27.97, 27.59, 27.52, 19.55. ${ }^{19} \mathrm{~F}$ NMR (377 MHz, $\left.\mathrm{CDCl}_{3}\right)$ $\delta$-63.16. HRMS (ESI): calcd for $\mathrm{C}_{27} \mathrm{H}_{29} \mathrm{~F}_{3} \mathrm{~N}_{2} \mathrm{NaO}_{4}[\mathrm{M}+\mathrm{Na}]^{+}$525.1972, found 525.1978. 


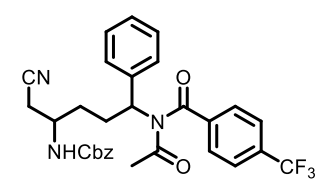

The compound was prepared according to the general procedure for reaction of oxime ester and styrene with petroleum ether/ethyl acetate as eluent. Yield: $41 \%$ (45.2 mg, white solid). ${ }^{1} \mathrm{H}$ NMR $\left(400 \mathrm{MHz}, \mathrm{CDCl}_{3}\right) \delta 7.69-7.61(\mathrm{~m}, 4 \mathrm{H}), 7.44-7.40(\mathrm{~m}, 2 \mathrm{H}), 7.39-7.19(\mathrm{~m}, 8 \mathrm{H}), 5.75(\mathrm{dd}, J=$ $16.1,8.1 \mathrm{~Hz}, 1 \mathrm{H}), 5.13(\mathrm{~s}, 2 \mathrm{H}), 3.99(\mathrm{~s}, 1 \mathrm{H}), 2.87-2.69(\mathrm{~m}, 1 \mathrm{H}), 2.65-2.47(\mathrm{~m}, 2 \mathrm{H}), 2.43-2.26$ $(\mathrm{m}, 1 \mathrm{H}), 1.88(\mathrm{~s}, 3 \mathrm{H}), 1.79-1.66(\mathrm{~m}, 3 \mathrm{H}) .{ }^{13} \mathrm{C} \mathrm{NMR}\left(101 \mathrm{MHz}, \mathrm{CDCl}_{3}\right) \delta 174.63,174.21,156.06$, $141.19,140.15,135.82(\mathrm{q}, J=32.7 \mathrm{~Hz}), 130.36,129.99,129.55,129.51,127.38(\mathrm{q}, J=3.7 \mathrm{~Hz})$, 124.70 (q, $J=124.70 \mathrm{~Hz}), 118.65,81.22,62.23,35.84,35.64,35.38,35.23,29.78,28.87,27.39$, 27.34. ${ }^{19} \mathrm{~F}$ NMR (377 MHz, $\left.\mathrm{CDCl}_{3}\right) \delta$-63.18. HRMS (ESI): calcd for $\mathrm{C}_{30} \mathrm{H}_{28} \mathrm{~F}_{3} \mathrm{~N}_{3} \mathrm{NaO}_{4}[\mathrm{M}+\mathrm{Na}]^{+}$ 574.1924, found 575.1925.

Tert-butyl 4-(3-( $N$-acetyl-4-(trifluoromethyl)benzamido)-3-phenylpropyl)-4(cyanomethyl)piperidine-1-carboxylate (45):

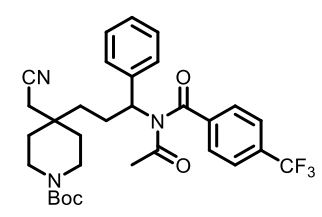

The compound was prepared according to the general procedure for reaction of oxime ester and styrene with petroleum ether/ethyl acetate as eluent. Yield: $68 \%\left(77.7 \mathrm{mg}\right.$, pale yellow solid). ${ }^{1} \mathrm{H}$ NMR $\left(400 \mathrm{MHz}, \mathrm{CDCl}_{3}\right) \delta 7.75-7.55(\mathrm{~m}, 4 \mathrm{H}), 7.44(\mathrm{~d}, J=7.4 \mathrm{~Hz}, 2 \mathrm{H}), 7.32(\mathrm{t}, J=7.3 \mathrm{~Hz}, 2 \mathrm{H})$, $7.29-7.26(\mathrm{~m}, 1 \mathrm{H}), 5.66(\mathrm{t}, J=7.5 \mathrm{~Hz}, 1 \mathrm{H}), 3.40-3.34(\mathrm{~m}, 3 \mathrm{H}), 2.51-2.34(\mathrm{~m}, 3 \mathrm{H}), 2.28-2.17$ $(\mathrm{m}, 1 \mathrm{H}), 1.89(\mathrm{~s}, 3 \mathrm{H}), 1.66-1.49(\mathrm{~m}, 8 \mathrm{H}), 1.45(\mathrm{~s}, 9 \mathrm{H}) .{ }^{13} \mathrm{C} \mathrm{NMR}\left(101 \mathrm{MHz}, \mathrm{CDCl}_{3}\right) \delta 173.28$, $172.90,154.68,139.74,138.68,134.41(\mathrm{q}, J=32.8 \mathrm{~Hz}), 128.99,128.64,128.18,126.06$ (q, $J=3.7$ $\mathrm{Hz}), 123.31(\mathrm{q}, J=271.2 \mathrm{~Hz}), 117.41,79.89,60.72,34.46,34.26,33.95,33.70,28.41,27.64,25.98$, 25.85. ${ }^{19} \mathrm{~F}$ NMR (377 MHz, $\left.\mathrm{CDCl}_{3}\right) \delta$-63.17. HRMS (ESI): calcd for $\mathrm{C}_{31} \mathrm{H}_{36} \mathrm{~F}_{3} \mathrm{~N}_{3} \mathrm{NaO}_{4}[\mathrm{M}+\mathrm{Na}]^{+}$ 594.2550, found 594.2562.

$N$-acetyl- $N$-(2-(2-(cyanomethyl)cyclopent-3-en-1-yl)-1-phenylethyl)-4(trifluoromethyl)benzamide (46):<smiles>CCC1(CC(C(C)=O)(c2ccccc2)N(C(C)=O)C(=O)c2ccc(Br)cc2)CCC1</smiles>

The compound was prepared according to the general procedure for reaction of oxime ester and styrene with petroleum ether/ethyl acetate as eluent. Yield: $54 \%$ (47.5 mg, white solid). ${ }^{1} \mathrm{H}$ NMR $\left(400 \mathrm{MHz}, \mathrm{CDCl}_{3}\right) \delta 7.70-7.61(\mathrm{~m}, 4 \mathrm{H}), 7.45(\mathrm{~d}, J=7.6 \mathrm{~Hz}, 2 \mathrm{H}), 7.34-7.29(\mathrm{~m}, 2 \mathrm{H}), 7.28-$ $7.24(\mathrm{~m}, 1 \mathrm{H}), 5.87-5.74(\mathrm{~m}, 2 \mathrm{H}), 5.72-5.58(\mathrm{~m}, 1 \mathrm{H}), 2.88-2.64(\mathrm{~m}, 3 \mathrm{H}), 2.53-2.39(\mathrm{~m}, 2 \mathrm{H})$, 
$2.32-1.96(\mathrm{~m}, 3 \mathrm{H}), 1.86(\mathrm{~s}, 3 \mathrm{H}) .{ }^{13} \mathrm{C}$ NMR $\left(101 \mathrm{MHz}, \mathrm{CDCl}_{3}\right) \delta$ 173.27, 173.25, 173.15, 172.48, 139.86, 139.82, 139.30, 138.18, 134.53 (q, $J=32.9 \mathrm{~Hz}), 134.68$ (q, $J=32.9 \mathrm{~Hz}), 132.65,132.48$, $130.75,130.57,129.00,128.59,128.56,128.16,128.03,128.00,126.10,126.07,126.03,125.99$, 125.91, $123.32(\mathrm{q}, J=272.1 \mathrm{~Hz}), 118.72,118.54,58.83,58.70,48.35,41.19,40.72,38.87,38.83$, 37.92, 37.23, 27.81, 27.64, 22.72, 22.47. $\left.{ }^{19} \mathrm{~F} \mathrm{NMR} \mathrm{(377} \mathrm{MHz,} \mathrm{CDCl}_{3}\right) \delta-63.17$. HRMS (ESI): calcd for $\mathrm{C}_{25} \mathrm{H}_{23} \mathrm{~F}_{3} \mathrm{~N}_{2} \mathrm{NaO}_{2}[\mathrm{M}+\mathrm{Na}]^{+} 463.1604$, found 463.1589 .

$N$-acetyl- $N$-(3-(2-cyanophenyl)-1-phenylpropyl)-4-(trifluoromethyl)benzamide (47):<smiles>CC(=O)N(C(=O)c1ccc(Br)cc1)C(CCc1ccccc1)(C(C)=O)c1ccccc1</smiles>

The compound was prepared according to the general procedure for reaction of oxime ester and styrene with petroleum ether/ethyl acetate as eluent. Yield: 53\% (47.7 mg, colorless oil). ${ }^{1} \mathrm{H}$ NMR $\left(400 \mathrm{MHz}, \mathrm{CDCl}_{3}\right) \delta 7.73-7.59(\mathrm{~m}, 5 \mathrm{H}), 7.53-7.45(\mathrm{~m}, 3 \mathrm{H}), 7.34-7.26(\mathrm{~m}, 5 \mathrm{H}), 5.83-5.66(\mathrm{~m}$, $1 \mathrm{H}), 2.99-2.86(\mathrm{~m}, 2 \mathrm{H}), 2.80-2.64(\mathrm{~m}, 2 \mathrm{H}), 1.94(\mathrm{~s}, 3 \mathrm{H}) .{ }^{13} \mathrm{C} \mathrm{NMR}\left(101 \mathrm{MHz}, \mathrm{CDCl}_{3}\right) \delta 173.23$, $172.98,145.03,139.79,138.54,134.18(\mathrm{q}, J=32.6 \mathrm{~Hz}), 132.92,129.50,128.93,128.59,128.26$, 128.05, $125.99(\mathrm{q}, J=3.6 \mathrm{~Hz}), 126.89,123.37(\mathrm{q}, J=271.0 \mathrm{~Hz}), 117.97,112.44,60.17,33.19$, 32.10, 27.47. ${ }^{19} \mathrm{~F}$ NMR (377 MHz, $\left.\mathrm{CDCl}_{3}\right) \delta$-63.11. HRMS (ESI): calcd for $\mathrm{C}_{26} \mathrm{H}_{21} \mathrm{~F}_{3} \mathrm{~N}_{2} \mathrm{NaO}_{2}$ $[\mathrm{M}+\mathrm{Na}]^{+} 473.1447$, found 473.1433 .

$N$-acetyl- $N$-(6-cyano-3,3-dimethyl-1-phenylhexyl)-4-(trifluoromethyl)benzamide (48):

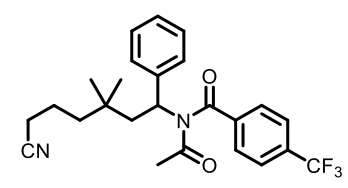

The compound was prepared according to the general procedure for reaction of oxime ester and styrene with petroleum ether/ethyl acetate as eluent. Yield: $53 \%$ (47.1 mg, colorless oil). ${ }^{1} \mathrm{H}$ NMR $\left(400 \mathrm{MHz}, \mathrm{CDCl}_{3}\right) \delta 7.64(\mathrm{~d}, J=8.0 \mathrm{~Hz}, 2 \mathrm{H}), 7.56(\mathrm{~d}, J=8.0 \mathrm{~Hz}, 2 \mathrm{H}), 7.51(\mathrm{~d}, J=7.3 \mathrm{~Hz}, 2 \mathrm{H})$, $7.33-7.23(\mathrm{~m}, 3 \mathrm{H}), 5.95-5.79(\mathrm{~m}, 1 \mathrm{H}), 2.52(\mathrm{dd}, J=14.8,7.2 \mathrm{~Hz}, 1 \mathrm{H}), 2.27-2.12(\mathrm{~m}, 3 \mathrm{H}), 1.79$ (s, 3H), $1.65-1.55(\mathrm{~m}, 2 \mathrm{H}), 1.43-1.29(\mathrm{~m}, 2 \mathrm{H}), 0.92$ (s, 3H), 0.90 (s, 3H). ${ }^{13} \mathrm{C}$ NMR $(101 \mathrm{MHz}$, $\left.\mathrm{CDCl}_{3}\right) \delta 173.28,172.42,140.23,140.19,134.29(\mathrm{q}, J=32.8 \mathrm{~Hz}), 128.88,128.78,128.45,127.98$, $126.00(\mathrm{q}, J=3.7 \mathrm{~Hz}), 123.32(\mathrm{q}, J=271.1 \mathrm{~Hz}), 119.64, \quad 57.37,43.69,41.37,33.30,28.01,27.27$, 27.23, 20.48, 17.73. ${ }^{19} \mathrm{~F}$ NMR (377 MHz, $\left.\mathrm{CDCl}_{3}\right) \delta$-63.16. HRMS (ESI): calcd for $\mathrm{C}_{25} \mathrm{H}_{27} \mathrm{~F}_{3} \mathrm{~N}_{2} \mathrm{NaO}_{2}[\mathrm{M}+\mathrm{Na}]^{+}$467.1917, found 467.1891.

$N$-acetyl- $N$-(5-cyano-1-(o-tolyl)pentyl)-4-(trifluoromethyl)benzamide (49):

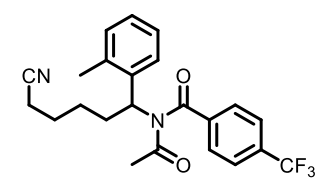

The compound was prepared according to the general procedure for reaction of oxime ester and styrene with petroleum ether/ethyl acetate as eluent. Yield: $89 \%$ (74.1 mg, colorless oil). ${ }^{1} \mathrm{H}$ NMR 
$\left(400 \mathrm{MHz}, \mathrm{CDCl}_{3}\right) \delta 7.62(\mathrm{~d}, J=8.3 \mathrm{~Hz}, 2 \mathrm{H}), 7.55(\mathrm{~d}, J=8.2 \mathrm{~Hz}, 2 \mathrm{H}), 7.35(\mathrm{~d}, J=7.7 \mathrm{~Hz}, 1 \mathrm{H})$, $7.17-7.03(\mathrm{~m}, 3 \mathrm{H}), 5.92(\mathrm{t}, J=7.7 \mathrm{~Hz}, 1 \mathrm{H}), 2.40(\mathrm{~s}, 3 \mathrm{H}), 2.38-2.20(\mathrm{~m}, 4 \mathrm{H}), 1.90(\mathrm{~s}, 3 \mathrm{H}), 1.80-$ $1.69(\mathrm{~m}, 2 \mathrm{H}), 1.56-1.45(\mathrm{~m}, 2 \mathrm{H}) .{ }^{13} \mathrm{C}$ NMR $\left(101 \mathrm{MHz}, \mathrm{CDCl}_{3}\right) \delta 173.39,172.21,139.85,136.83$, $135.87,134.30(\mathrm{q}, J=32.7 \mathrm{~Hz}), 130.78,128.90,128.35,128.04,125.93,125.88(\mathrm{q}, J=3.7 \mathrm{~Hz})$, $123.32(\mathrm{q}, J=271.0 \mathrm{~Hz}), 119.44,56.38,31.97,27.07,25.98,25.12,19.59,17.04 .{ }^{19} \mathrm{~F}$ NMR $(377$ $\left.\mathrm{MHz}, \mathrm{CDCl}_{3}\right) \delta$-63.11. HRMS (ESI): calcd for $\mathrm{C}_{23} \mathrm{H}_{23} \mathrm{~F}_{3} \mathrm{~N}_{2} \mathrm{NaO}_{2}[\mathrm{M}+\mathrm{Na}]^{+} 439.1604$, found 439.1598 .

$N$-acetyl- $N$-(5-cyano-1-(m-tolyl)pentyl)-4-(trifluoromethyl)benzamide (50):<smiles>CCCCC(c1ccc(C)cc1)(c1ccc(C)cc1)N(C(C)=O)C(C)=O</smiles>

The compound was prepared according to the general procedure for reaction of oxime ester and styrene with petroleum ether/ethyl acetate as eluent. Yield: $71 \%$ (59.1 mg, colorless oil). ${ }^{1} \mathrm{H}$ NMR $\left(400 \mathrm{MHz}, \mathrm{CDCl}_{3}\right) \delta 7.71-7.60(\mathrm{~m}, 4 \mathrm{H}), 7.24-7.16(\mathrm{~m}, 3 \mathrm{H}), 7.06(\mathrm{~d}, J=6.8 \mathrm{~Hz}, 1 \mathrm{H}), 5.67(\mathrm{t}, J$ $=7.8 \mathrm{~Hz}, 1 \mathrm{H}), 2.43-2,34(\mathrm{~m}, 3 \mathrm{H}), 2.32(\mathrm{~s}, 3 \mathrm{H}), 2.28-2.22(\mathrm{~m}, 1 \mathrm{H}), 1.90(\mathrm{~s}, 3 \mathrm{H}), 1.74(\mathrm{tt}, J=14.0$, $7.0 \mathrm{~Hz}, 2 \mathrm{H}), 1.58-1.49(\mathrm{~m}, 2 \mathrm{H}) .{ }^{13} \mathrm{C} \mathrm{NMR}\left(101 \mathrm{MHz}, \mathrm{CDCl}_{3}\right) \delta 173.22,172.94,139.85,138.81$, $138.17,134.23$ (q, $J=32.8 \mathrm{~Hz}), 128.96,128.79,128.71,128.42,125.96$ (q, $J=3.6 \mathrm{~Hz}), 125.13$, $123.35(\mathrm{q}, J=271.1 \mathrm{~Hz}), 119.43,60.09,31.30,27.49,26.10,25.08,21.47,17.08 .{ }^{19} \mathrm{~F}$ NMR $(377$ $\left.\mathrm{MHz}, \mathrm{CDCl}_{3}\right) \delta-63.15$. HRMS (ESI): calcd for $\mathrm{C}_{23} \mathrm{H}_{23} \mathrm{~F}_{3} \mathrm{~N}_{2} \mathrm{NaO}_{2}[\mathrm{M}+\mathrm{Na}]^{+} 439.1604$, found 439.1600 .

$N$-acetyl- $N$-(5-cyano-1-(p-tolyl)pentyl)-4-(trifluoromethyl)benzamide (51):

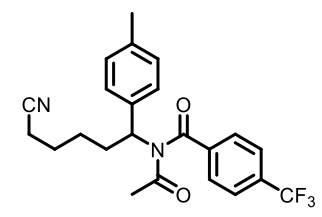

The compound was prepared according to the general procedure for reaction of oxime ester and styrene with petroleum ether/ethyl acetate as eluent. Yield: $56 \%$ (46.6 mg, colorless oil). ${ }^{1} \mathrm{H}$ NMR $\left(400 \mathrm{MHz}, \mathrm{CDCl}_{3}\right) \delta 7.76-7.57(\mathrm{~m}, 4 \mathrm{H}), 7.31(\mathrm{~d}, J=7.8 \mathrm{~Hz}, 2 \mathrm{H}), 7.11(\mathrm{~d}, J=7.7 \mathrm{~Hz}, 2 \mathrm{H}), 5.67$ $(\mathrm{t}, J=7.8 \mathrm{~Hz}, 1 \mathrm{H}), 2.43-2.33(\mathrm{~m}, 3 \mathrm{H}), 2.32-2.24(\mathrm{~m}, 4 \mathrm{H}), 1.88(\mathrm{~s}, 3 \mathrm{H}), 1.75(\mathrm{tt}, J=14.2,6.9 \mathrm{~Hz}$, $2 \mathrm{H}), 1.58-1.49(\mathrm{~m}, 2 \mathrm{H}) .{ }^{13} \mathrm{C} \mathrm{NMR}\left(101 \mathrm{MHz}, \mathrm{CDCl}_{3}\right) \delta 173.21,172.88,139.90,137.71,135.78$, $134.23(\mathrm{q}, J=32.7 \mathrm{~Hz}), 129.21,128.96,128.06,125.98(\mathrm{q}, J=3.6 \mathrm{~Hz}), 123.35$ (q, $J=171.1 \mathrm{~Hz})$, $119.43,59.95,31.33,27.54,26.13,25.09,21.05,17.09 .{ }^{19} \mathrm{~F} \mathrm{NMR}\left(377 \mathrm{MHz}, \mathrm{CDCl}_{3}\right) \delta-63.13$. HRMS (ESI): calcd for $\mathrm{C}_{23} \mathrm{H}_{23} \mathrm{~F}_{3} \mathrm{~N}_{2} \mathrm{NaO}_{2}[\mathrm{M}+\mathrm{Na}]^{+} 439.1604$, found 439.1590 .

4-(1-( $N$-acetyl-4-(trifluoromethyl)benzamido)-5-cyanopentyl)phenyl acetate (52):<smiles>CCCCC(c1ccc(C)cc1)(c1ccc(O)cc1)N(C(C)=O)C(C)=O</smiles> 
The compound was prepared according to the general procedure for reaction of oxime ester and styrene with petroleum ether/ethyl acetate as eluent. Yield: 55\% (50.6 mg, colorless oil). ${ }^{1} \mathrm{H}$ NMR $\left(400 \mathrm{MHz}, \mathrm{CDCl}_{3}\right) \delta 7.74-7.62(\mathrm{~m}, 4 \mathrm{H}), 7.46(\mathrm{~d}, J=8.3 \mathrm{~Hz}, 2 \mathrm{H}), 7.04(\mathrm{~d}, J=8.3 \mathrm{~Hz}, 2 \mathrm{H}), 5.70$ $(\mathrm{t}, J=7.7 \mathrm{~Hz}, 1 \mathrm{H}), 2.48-2.40(\mathrm{~m}, 1 \mathrm{H}), 2.36(\mathrm{t}, J=6.9 \mathrm{~Hz}, 2 \mathrm{H}), 2.29-2.19(\mathrm{~m}, 4 \mathrm{H}), 1.87(\mathrm{~s}, 3 \mathrm{H})$, $1.73(\mathrm{tt}, J=15.1,7.6 \mathrm{~Hz}, 2 \mathrm{H}), 1.53(\mathrm{tt}, J=14.6,7.3 \mathrm{~Hz}, 2 \mathrm{H}) .{ }^{13} \mathrm{C} \mathrm{NMR}\left(101 \mathrm{MHz}, \mathrm{CDCl}_{3}\right) \delta 173.05$, $172.86,169.18,150.25,139.78,136.48,134.4$ (q, $J=32.8 \mathrm{~Hz}), 129.36,128.96,126.1$ (q, $J=3.6$ $\mathrm{Hz}), 123.3(\mathrm{q}, J=123.3 \mathrm{~Hz}) 119.33,59.62,31.31,27.69,26.07,25.04,21.08,17.07 .19$ F NMR (377 $\left.\mathrm{MHz}, \mathrm{CDCl}_{3}\right) \delta$-63.16. HRMS (ESI): calcd for $\mathrm{C}_{24} \mathrm{H}_{23} \mathrm{~F}_{3} \mathrm{~N}_{2} \mathrm{NaO}_{4}[\mathrm{M}+\mathrm{Na}]^{+} 483.1502$, found 483.1487.

$N$-acetyl- $N$-(5-cyano-1-(4-fluorophenyl)pentyl)-4-(trifluoromethyl)benzamide (53):

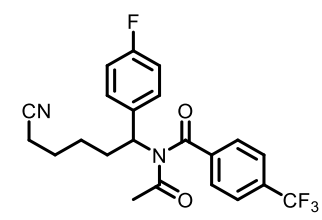

The compound was prepared according to the general procedure for reaction of oxime ester and styrene with petroleum ether/ethyl acetate as eluent. Yield: $63 \%$ (52.9 mg, colorless oil). ${ }^{1} \mathrm{H}$ NMR $\left(400 \mathrm{MHz}, \mathrm{CDCl}_{3}\right) \delta 7.72-7.73(\mathrm{~m}, 4 \mathrm{H}), 7.43(\mathrm{dd}, J=8.4,5.5 \mathrm{~Hz}, 2 \mathrm{H}), 7.03-6.96(\mathrm{~m}, 2 \mathrm{H}), 5.69(\mathrm{t}$, $J=7.9 \mathrm{~Hz}, 1 \mathrm{H}), 2.47-2.33(\mathrm{~m}, 3 \mathrm{H}), 2.28-2.21(\mathrm{~m}, 1 \mathrm{H}), 1.85(\mathrm{~s}, 3 \mathrm{H}), 1.78-1.69(\mathrm{~m}, 2 \mathrm{H}), 1.56-$ $1.48(\mathrm{~m}, 2 \mathrm{H}) .{ }^{13} \mathrm{C}$ NMR $\left(101 \mathrm{MHz}, \mathrm{CDCl}_{3}\right) \delta 173.01,172.81,162.23(\mathrm{~d}, J=245.5 \mathrm{~Hz}), 139.76$, $134.72(\mathrm{~d}, J=3.1 \mathrm{~Hz}), 134.46(\mathrm{q}, J=32.8 \mathrm{~Hz}), 130.06(\mathrm{~d}, J=8.1 \mathrm{~Hz}), 128.97,126.11(\mathrm{q}, J=3.6$ $\mathrm{Hz}), 123.29$ (q, $J=271.1 \mathrm{~Hz}), 119.35,115.39$ (d, $J=21.2 \mathrm{~Hz}), 59.54,31.38,27.73,26.08,25.02$, 17.08. ${ }^{19} \mathrm{~F}$ NMR $\left(377 \mathrm{MHz}, \mathrm{CDCl}_{3}\right) \delta-63.17$ (s), -113.91 (s). HRMS (ESI): calcd for $\mathrm{C}_{22} \mathrm{H}_{20} \mathrm{~F}_{4} \mathrm{~N}_{2} \mathrm{NaO}_{2}[\mathrm{M}+\mathrm{Na}]^{+} 443.1353$, found 443.1342.

$N$-acetyl- $N$-(1-(4-chlorophenyl)-5-cyanopentyl)-4-(trifluoromethyl)benzamide (54):

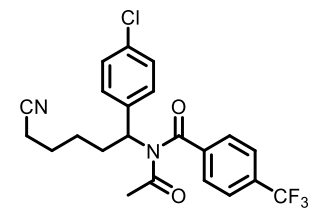

The compound was prepared according to the general procedure for reaction of oxime ester and styrene with petroleum ether/ethyl acetate as eluent. Yield: $54 \%$ (47.2 mg, colorless oil). ${ }^{1} \mathrm{H}$ NMR $\left(400 \mathrm{MHz}, \mathrm{CDCl}_{3}\right) \delta 7.73-7.63(\mathrm{~m}, 4 \mathrm{H}), 7.40(\mathrm{~d}, J=8.4 \mathrm{~Hz}, 2 \mathrm{H}), 7.28(\mathrm{~d}, J=8.4 \mathrm{~Hz}, 2 \mathrm{H}), 5.68$ $(\mathrm{t}, J=7.8 \mathrm{~Hz}, 1 \mathrm{H}), 2.47-2.33(\mathrm{~m}, 3 \mathrm{H}), 2.28-2.18(\mathrm{~m}, 1 \mathrm{H}), 1.85(\mathrm{~s}, 3 \mathrm{H}), 1.79-1.69(\mathrm{~m}, 2 \mathrm{H}), 1.56$ $-1.48(\mathrm{~m}, 2 \mathrm{H}) .{ }^{13} \mathrm{C} \mathrm{NMR}\left(101 \mathrm{MHz}, \mathrm{CDCl}_{3}\right) \delta 172.92,172.87,139.69,137.44,134.51$ (q, $J=32.7$ $\mathrm{Hz}), 133.82,129.67,128.98,128.70,126.15(\mathrm{q}, J=3.5 \mathrm{~Hz}), 123.28$ (q, $J=271.1 \mathrm{~Hz}), 119.34,59.56$, 31.14, 27.79, 26.04, 25.01, 17.09. ${ }^{19} \mathrm{~F}$ NMR (377 MHz, $\left.\mathrm{CDCl}_{3}\right) \delta-63.17$. HRMS (ESI): calcd for $\mathrm{C}_{22} \mathrm{H}_{20} \mathrm{ClF}_{3} \mathrm{~N}_{2} \mathrm{NaO}_{2}[\mathrm{M}+\mathrm{Na}]^{+} 459.1058$, found 459.1047 .

$N$-acetyl- $N$-(1-(4-bromophenyl)-5-cyanopentyl)-4-(trifluoromethyl)benzamide (55): 


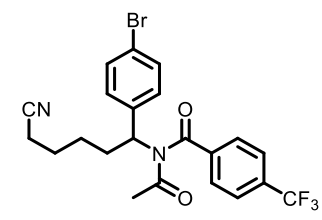

The compound was prepared according to the general procedure for reaction of oxime ester and styrene with petroleum ether/ethyl acetate as eluent. Yield: $62 \%\left(59.6 \mathrm{mg}\right.$, pale yellow solid). ${ }^{1} \mathrm{H}$ $\operatorname{NMR}\left(400 \mathrm{MHz} \mathrm{CDCl}_{3}\right) \delta 7.72(\mathrm{~d}, \mathrm{~J}=8.6 \mathrm{~Hz}, 2 \mathrm{H}), 7.68(\mathrm{~d}, J=8.5 \mathrm{~Hz}, 2 \mathrm{H}), 7.46(\mathrm{~d}, J=8.4 \mathrm{~Hz}$, $2 \mathrm{H}), 7.35(\mathrm{~d}, J=8.4 \mathrm{~Hz}, 2 \mathrm{H}), 5.67(\mathrm{t}, J=7.8 \mathrm{~Hz}, 1 \mathrm{H}), 2.48-2.35(\mathrm{~m}, 3 \mathrm{H}), 2.25(\mathrm{tt}, J=14.7,7.3$ $\mathrm{Hz}, 1 \mathrm{H}), 1.87(\mathrm{~s}, 3 \mathrm{H}), 1.76(\mathrm{tt}, J=14.9,7.3 \mathrm{~Hz}, 2 \mathrm{H}), 1.58-1.50(\mathrm{~m}, 2 \mathrm{H}) .{ }^{13} \mathrm{C} \mathrm{NMR}(101 \mathrm{MHz}$, $\left.\mathrm{CDCl}_{3}\right) \delta 172.89,172.84,139.68,137.98,134.53$ (q, $\left.J=32.8 \mathrm{~Hz}\right), 131.67,129.99,128.97,126.15$ $(\mathrm{q}, J=3.6 \mathrm{~Hz}), 123.27(\mathrm{q}, J=271.1 \mathrm{~Hz}), 122.01,119.21,59.62,31.08,27.80,26.04,25.01,17.09 .{ }^{19} \mathrm{~F}$ NMR (377 MHz, $\left.\mathrm{CDCl}_{3}\right) \delta$-63.16. HRMS (ESI): calcd for $\mathrm{C}_{22} \mathrm{H}_{20} \mathrm{BrF}_{3} \mathrm{~N}_{2} \mathrm{NaO}_{2}[\mathrm{M}+\mathrm{Na}]^{+} 503.0552$, 505.0532, found 503.0557, 505.0538.

$N$-(1-([1,1'-biphenyl]-4-yl)-5-cyanopentyl)- $N$-acetyl-4-(trifluoromethyl)benzamide (56):

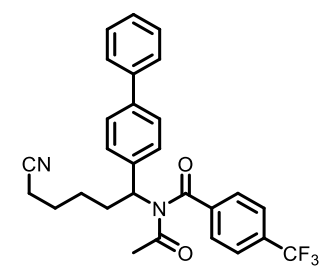

The compound was prepared according to the general procedure for reaction of oxime ester and styrene with petroleum ether/ethyl acetate as eluent. Yield: $54 \%$ (51.6 mg, pale yellow solid). ${ }^{1} \mathrm{H}$ NMR (400 MHz, $\left.\mathrm{CDCl}_{3}\right) \delta 7.74-7.66(\mathrm{~m}, 4 \mathrm{H}), 7.58-7.49(\mathrm{~m}, 6 \mathrm{H}), 7.45-7.40(\mathrm{~m}, 2 \mathrm{H}), 7.34(\mathrm{t}$, $J=7.2 \mathrm{~Hz}, 1 \mathrm{H}), 5.76(\mathrm{t}, J=7.8 \mathrm{~Hz}, 1 \mathrm{H}), 2.54-2.42(\mathrm{~m}, 1 \mathrm{H}), 2.41-2.22(\mathrm{~m}, 3 \mathrm{H}), 1.92(\mathrm{~s}, 3 \mathrm{H})$, $1.84-1.70(\mathrm{~m}, 2 \mathrm{H}), 1.64-1.53(\mathrm{~m}, 2 \mathrm{H}) .{ }^{13} \mathrm{C} \mathrm{NMR}\left(101 \mathrm{MHz}, \mathrm{CDCl}_{3}\right) \delta 173.18,172.99,140.79$, $140.41,139.83,137.86,134.86,134.54,134.21,133.88,130.53,129.00,128.79,128.59,127.46$, 127.20, 127.02, 126.11, 126.08, 126.04, 126.01, 124.68, 121.97, 119.40, 119.26, 59.95, 31.28, 27.66, 26.14, 25.10, 17.11. ${ }^{19} \mathrm{~F}$ NMR (377 MHz, $\left.\mathrm{CDCl}_{3}\right) \delta$-63.13. HRMS (ESI): calcd for $\mathrm{C}_{28} \mathrm{H}_{25} \mathrm{~F}_{3} \mathrm{~N}_{2} \mathrm{NaO}_{2}$ $[\mathrm{M}+\mathrm{Na}]^{+}$501.1760, found 501.1747.

\section{2,2,6,6-Tetramethylpiperidin-1-yl benzoate (57):}<smiles>CC1(C)CCCC(C)(C)N1OC(=O)c1ccccc1</smiles>

The compound was prepared according to the procedure for capture of reaction intermediates and isolated with petroleum ether/ethyl acetate as eluent. ${ }^{1} \mathrm{H}$ NMR Yield: 97\%, white solid. ${ }^{1} \mathrm{H}$ NMR $\left(400 \mathrm{MHz} \mathrm{CDCl}_{3}\right) \delta 8.08(\mathrm{~d}, J=7.6 \mathrm{~Hz}, 2 \mathrm{H}), 7.56(\mathrm{t}, J=7.3 \mathrm{~Hz}, 1 \mathrm{H}), 7.46(\mathrm{t}, J=7.4 \mathrm{~Hz}, 2 \mathrm{H}), 1.84$ $-1.65(\mathrm{~m}, 3 \mathrm{H}), 1.63-1.53(\mathrm{~m}, 2 \mathrm{H}), 1.49-1.42(\mathrm{~m}, 1 \mathrm{H}), 1.27(\mathrm{~s}, 6 \mathrm{H}), 1.13(\mathrm{~s}, 6 \mathrm{H}) .{ }^{13} \mathrm{C} \mathrm{NMR}(101$ $\left.\mathrm{MHz}_{,} \mathrm{CDCl}_{3}\right) \delta 166.37,132.83,129.78,129.57,128.44,60.42,39.10,32.00,20.87,17.03$. HRMS (ESI): calcd for $\mathrm{C}_{14} \mathrm{H}_{24} \mathrm{NO}_{2}[\mathrm{M}+\mathrm{H}]^{+}$262.1802, found 262.1794. calcd for $\mathrm{C}_{14} \mathrm{H}_{23} \mathrm{NNaO}_{2}[\mathrm{M}+\mathrm{H}]^{+}$ 284.1621, found 282.1612.

3-Ethoxy-1,3-diphenylpropan-1-one (59): 


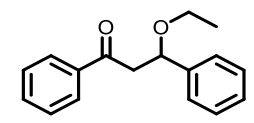

The compound was prepared according to the procedure for capture of reaction intermediates with petroleum ether/ethyl acetate as eluent. Yield: $54 \%$ (27.4 mg, colorless oil). ${ }^{1} \mathrm{H}$ NMR (400 MHz, $\left.\mathrm{CDCl}_{3}\right) \delta 7.93(\mathrm{~d}, J=7.7 \mathrm{~Hz}, 2 \mathrm{H}), 7.53(\mathrm{t}, J=7.3 \mathrm{~Hz}, 1 \mathrm{H}), 7.46-7.39(\mathrm{~m}, 4 \mathrm{H}), 7.35(\mathrm{t}, J=7.3 \mathrm{~Hz}$, $2 \mathrm{H}), 7.30-7.25(\mathrm{~m}, 1 \mathrm{H}), 4.99(\mathrm{dd}, J=8.0,4.6 \mathrm{~Hz}, 1 \mathrm{H}), 3.59(\mathrm{dd}, J=16.4,8.2 \mathrm{~Hz}, 1 \mathrm{H}), 3.40(\mathrm{q}, J$ $=7.0 \mathrm{~Hz}, 2 \mathrm{H}), 3.09(\mathrm{dd}, J=16.4,4.5 \mathrm{~Hz}, 1 \mathrm{H}), 1.12(\mathrm{t}, J=7.0 \mathrm{~Hz}, 3 \mathrm{H}) .{ }^{13} \mathrm{C} \mathrm{NMR}\left(101 \mathrm{MHz}, \mathrm{CDCl}_{3}\right)$ $\delta 199.18,143.75,138.94,134.25,129.86,129.83,129.61,129.02,127.94,79.21,65.85,48.88$, 16.53. HRMS (ESI): calcd for $\mathrm{C}_{17} \mathrm{H}_{18} \mathrm{NNaO}_{2}[\mathrm{M}+\mathrm{Na}]^{+} 277.1199$, found 277.1190. 


\section{9. ${ }^{1} \mathrm{H},{ }^{13} \mathrm{C}$ and ${ }^{19} \mathrm{~F}$ spectra of All Products}

$N$-acetyl- $N$-(3-oxo-1,3-diphenylpropyl)acetamide (3):<smiles>CC(=O)C(c1ccccc1)N(C(C)=O)C(C)=O</smiles>

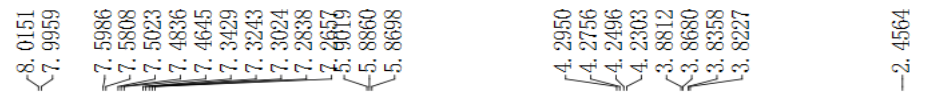
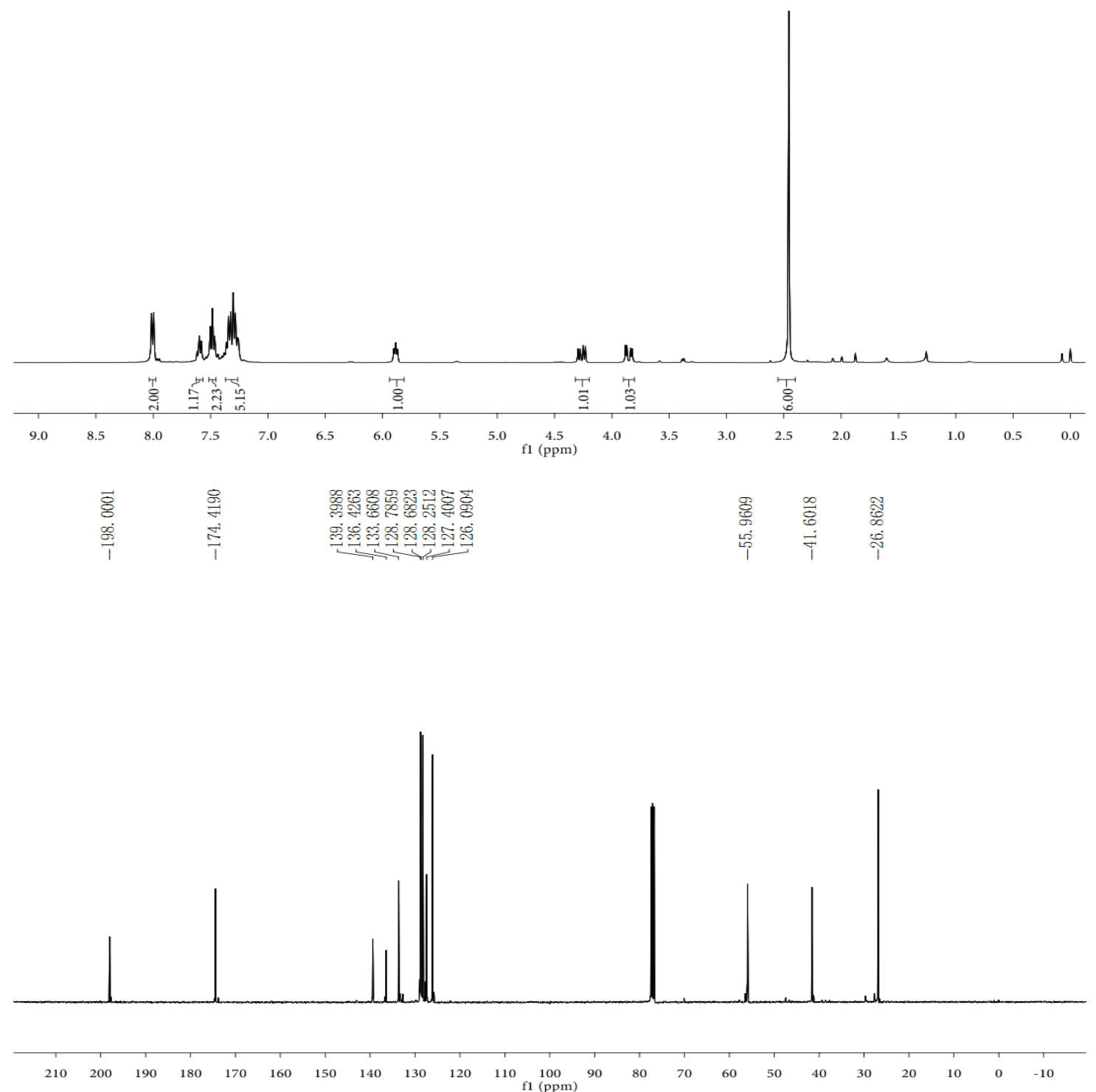
$N$-acetyl- $N$-(3-oxo-1-phenyl-3-(o-tolyl)propyl)acetamide (4):<smiles>CC(=O)N(C(C)=O)C(CC(=O)c1ccccc1C)c1ccccc1</smiles>

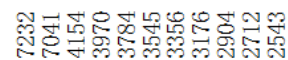

riningivitis

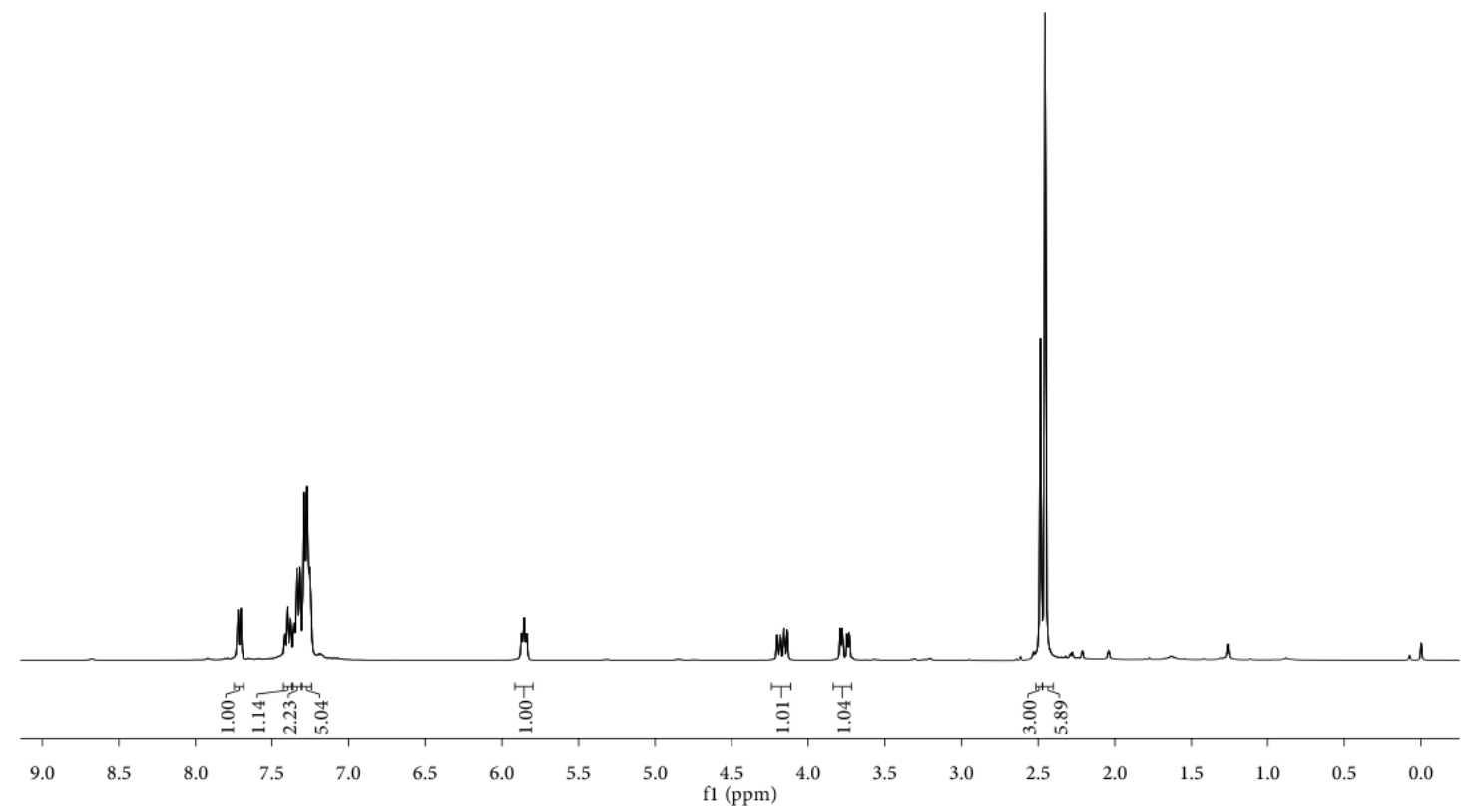

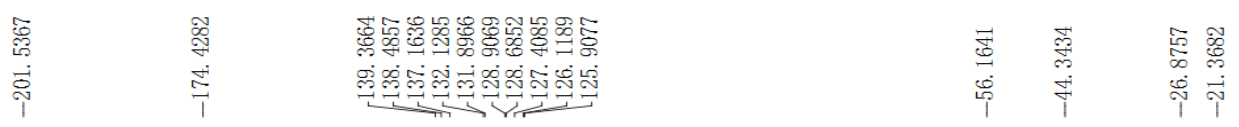

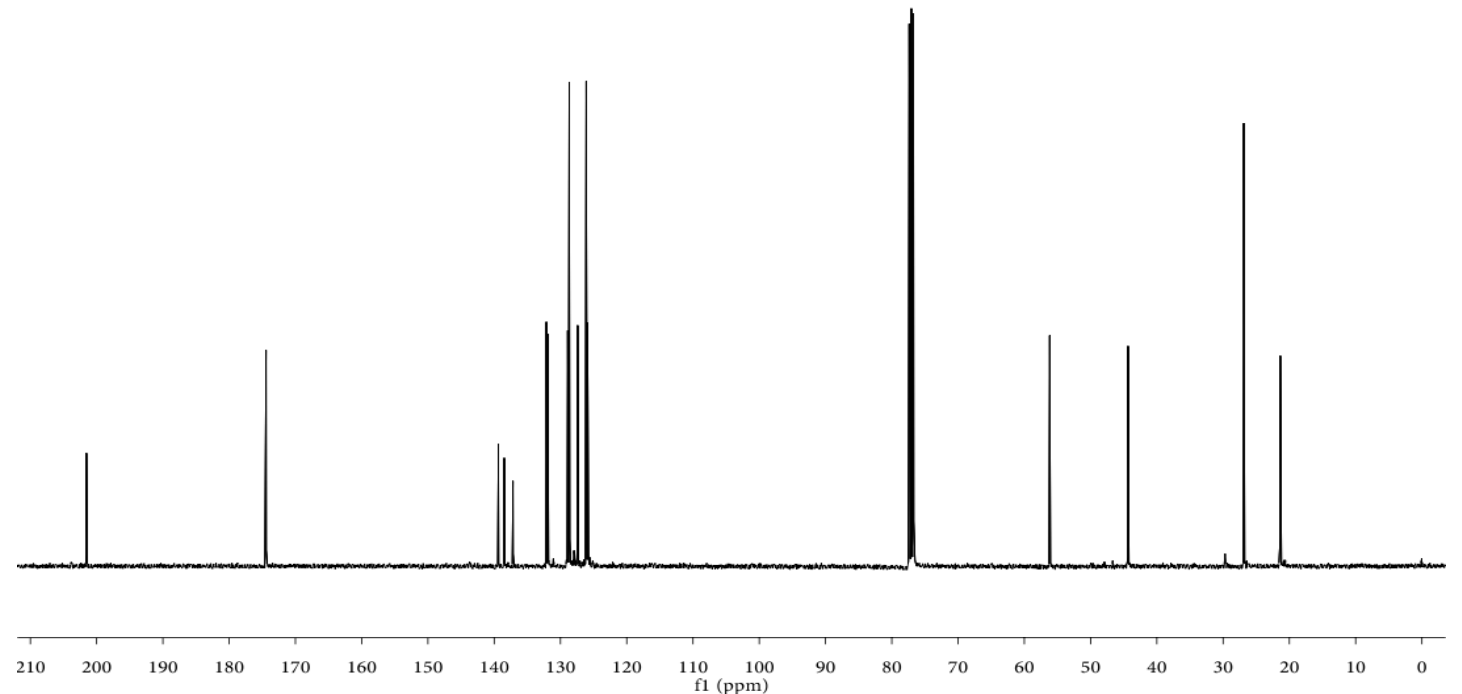


$N$-acetyl- $N$-(3-oxo-1-phenyl-3-(m-tolyl)propyl)acetamide (5):

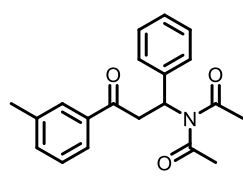

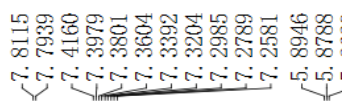

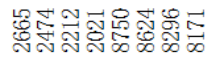

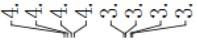

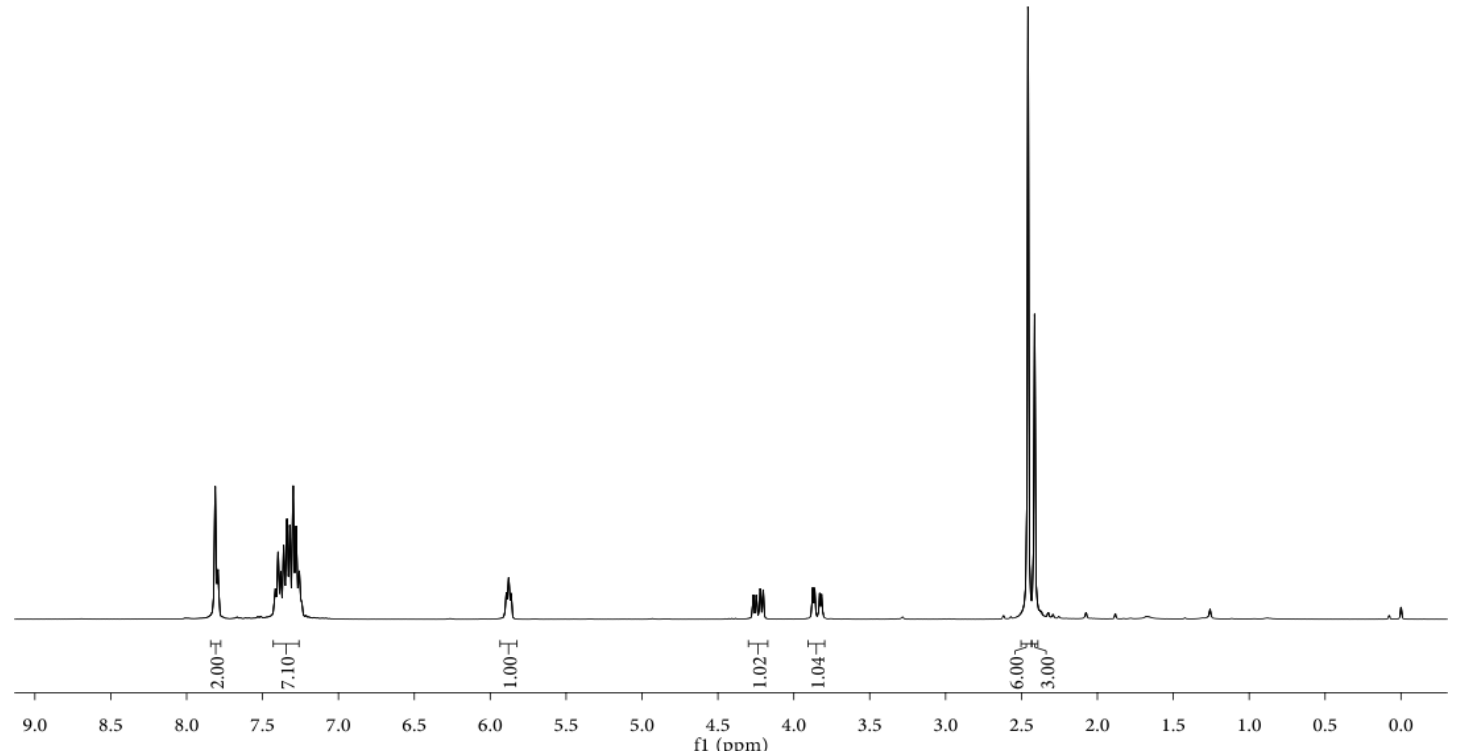

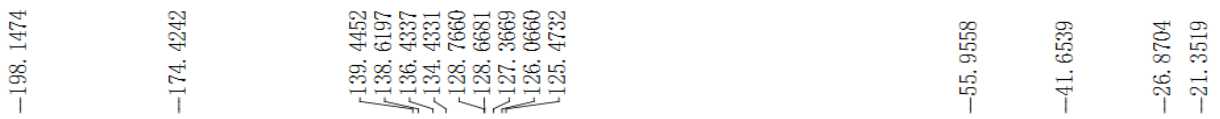

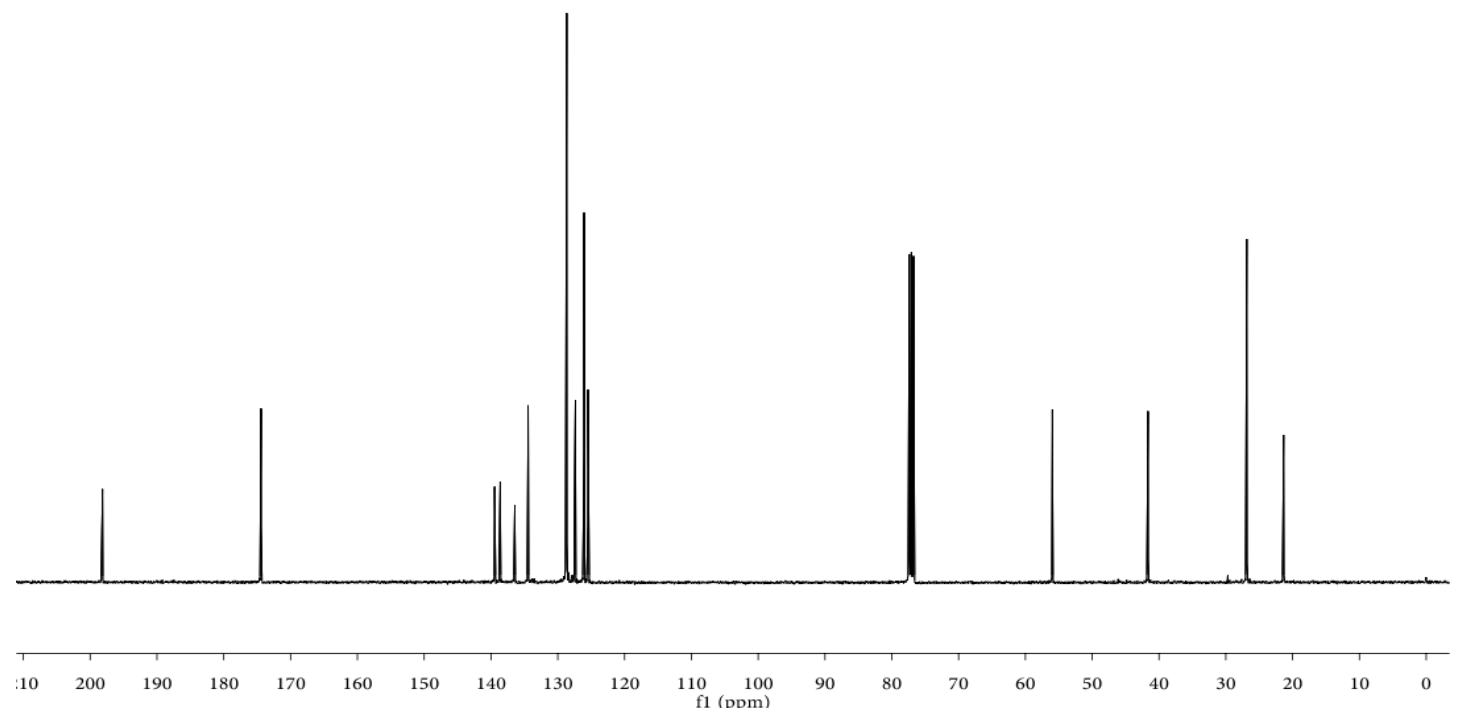


$N$-acetyl- $N$-(3-oxo-1-phenyl-3-(p-tolyl)propyl)acetamide (6):<smiles>[Te]</smiles>

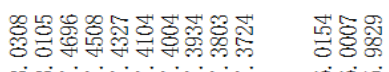

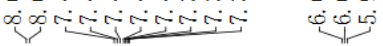

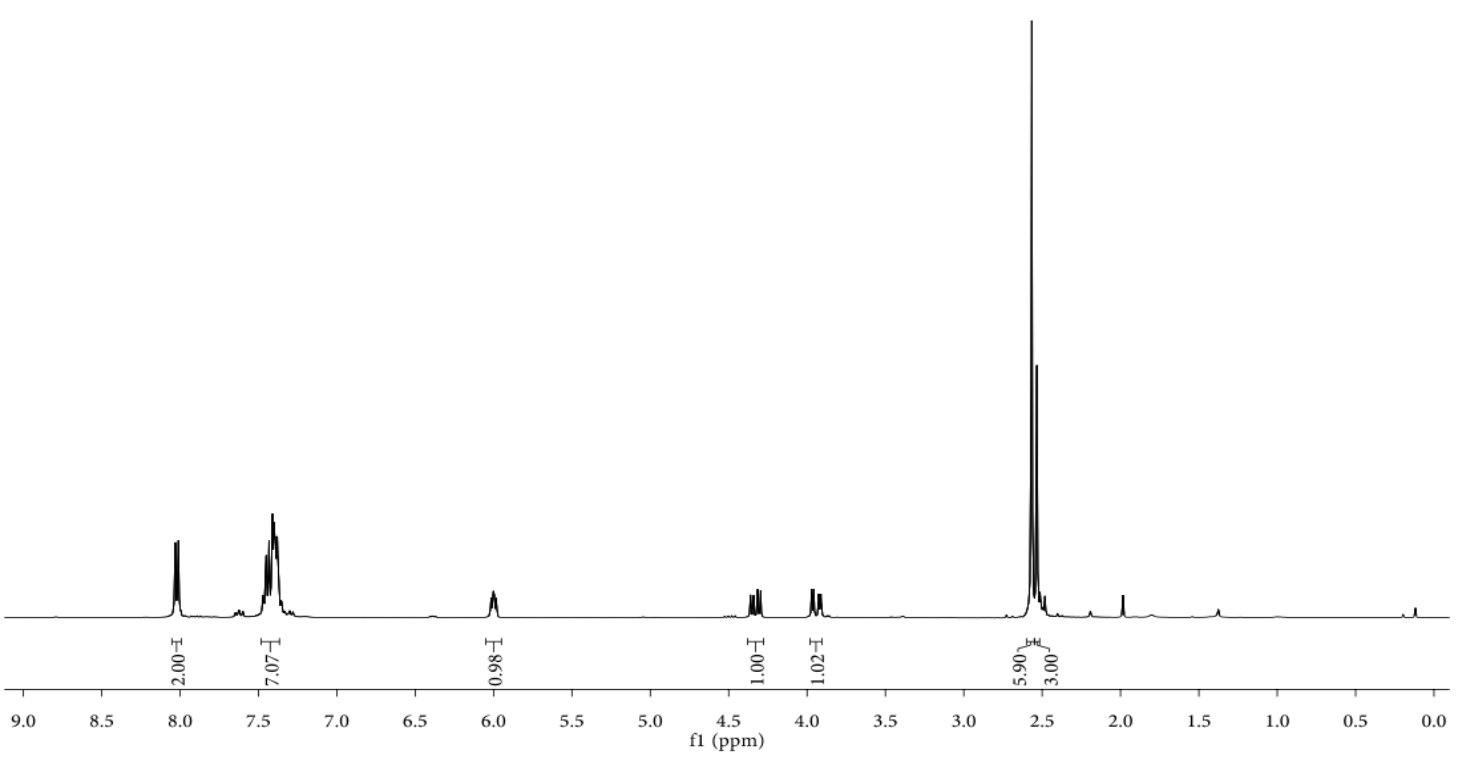

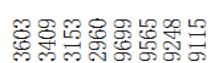

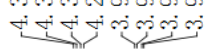

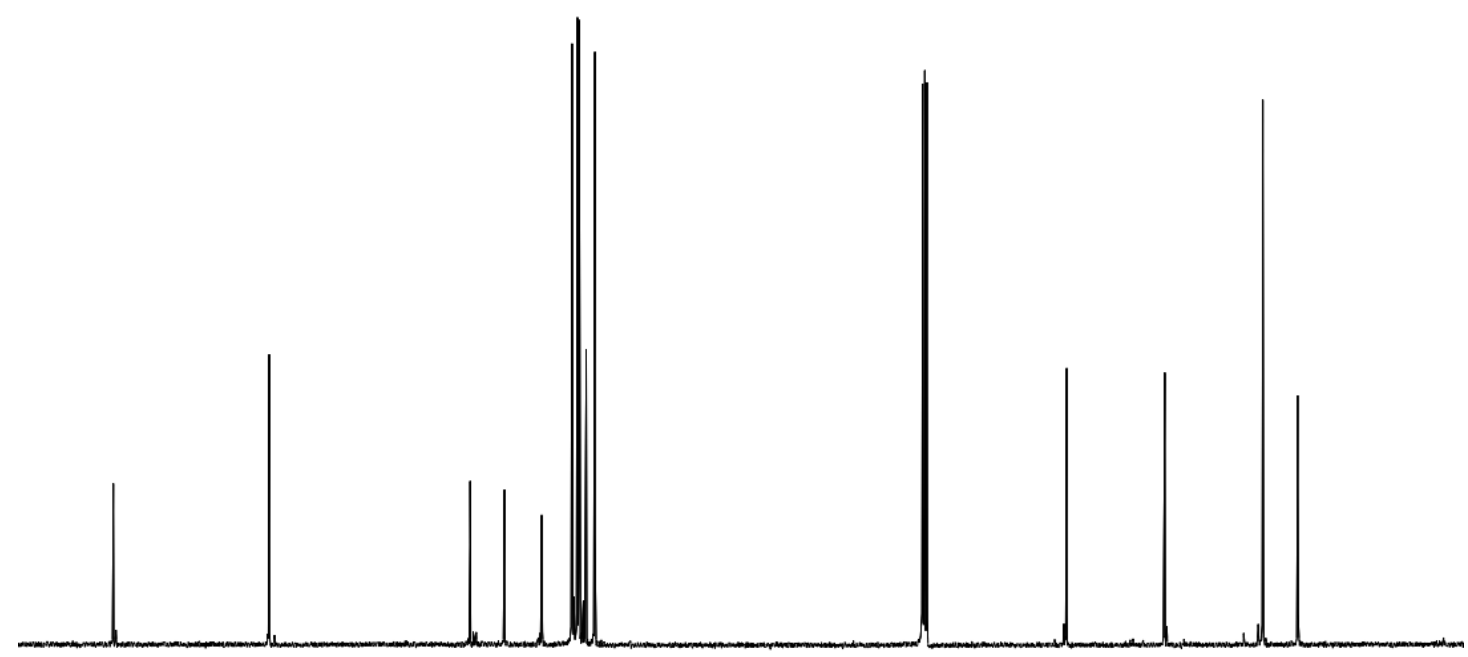

$\begin{array}{rllllllllll}210 & 200 & 190 & 180 & 170 & 160 & 150 & 140 & 130 & 120 & \begin{array}{l}110 \\ \mathrm{f} 1(\mathrm{ppm})\end{array}\end{array}$ 
$N$-acetyl- $N$-(3-(4-chlorophenyl)-3-oxo-1-phenylpropyl)acetamide (7):<smiles>CC(=O)N(C(C)=O)C(CC(=O)c1ccc(Cl)cc1)c1ccccc1</smiles>

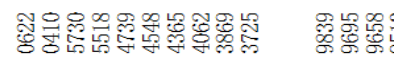

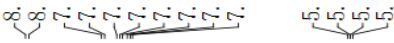

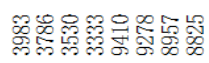

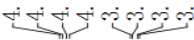
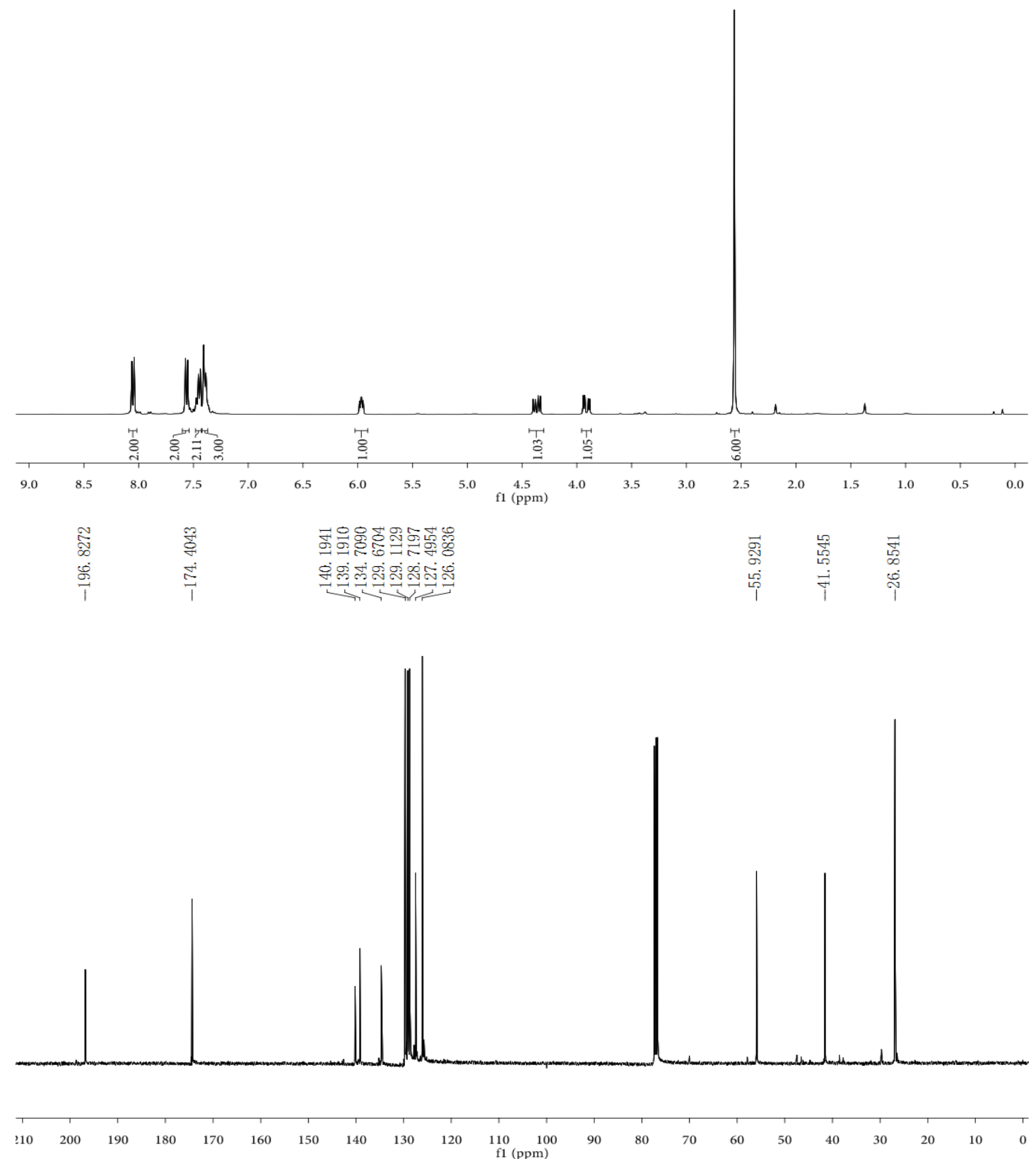
$N$-acetyl- $N$-(3-(4-methoxyphenyl)-3-oxo-1-phenylpropyl)acetamide (8):

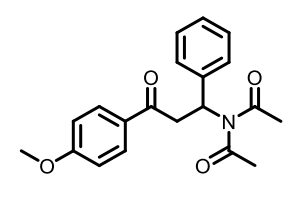

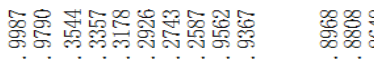

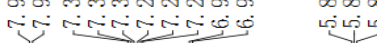

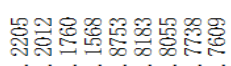

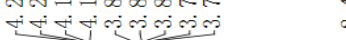

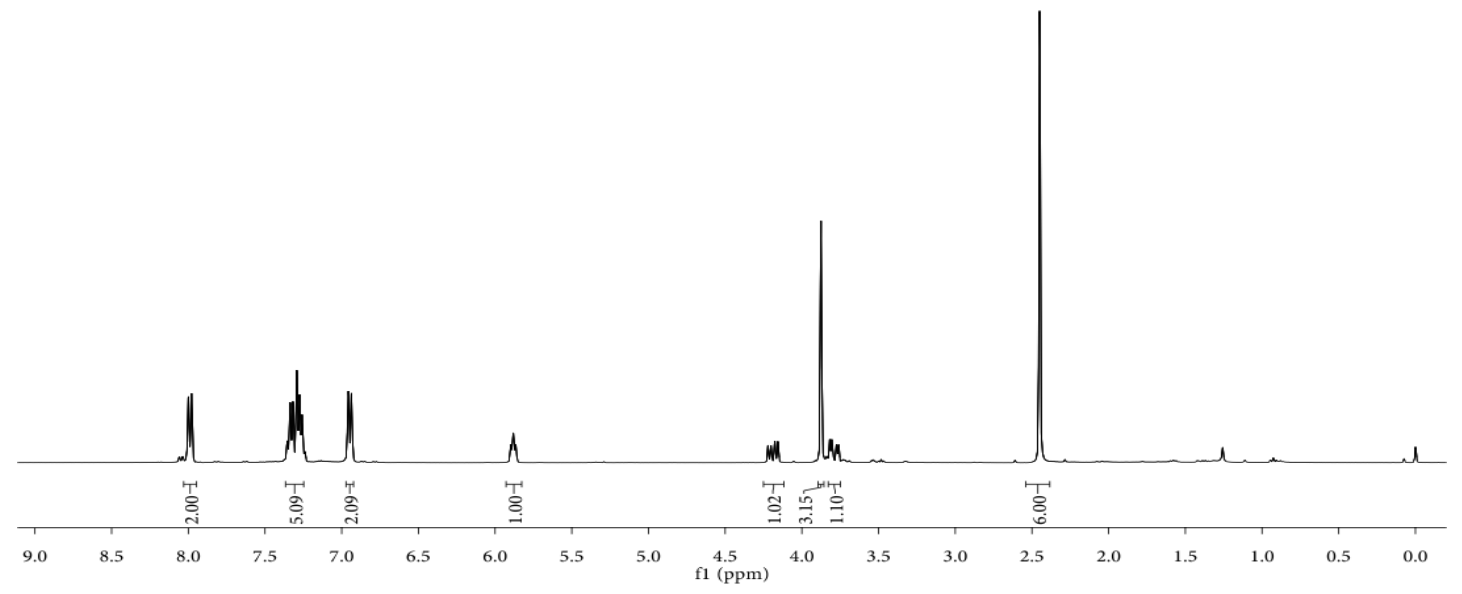

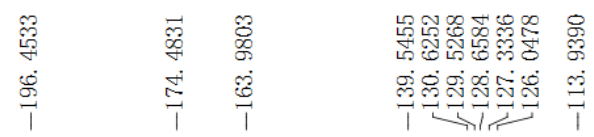

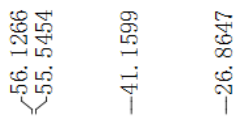

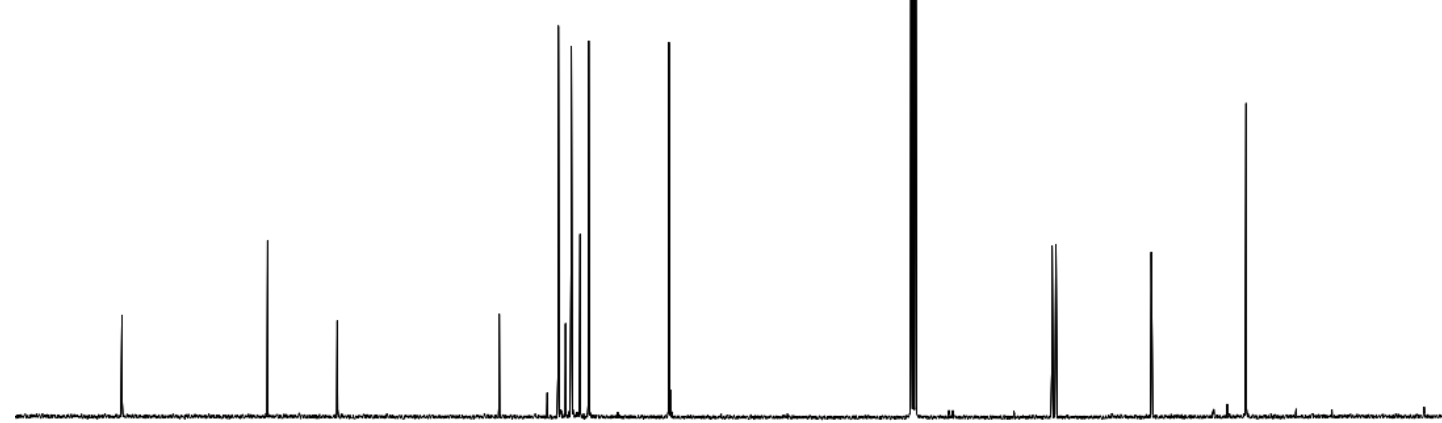

210

$\begin{array}{llllllllll}200 & 190 & 180 & 170 & 160 & 150 & 140 & 130 & 120 & \begin{array}{c}110 \\ \text { f1 (ppm) }\end{array}\end{array}$ 
$N$-acetyl- $N$-(3-(furan-2-yl)-3-oxo-1-phenylpropyl)acetamide(9):<smiles>CC(=O)N(C(C)=O)C(CC(=O)c1ccco1)c1ccccc1</smiles>

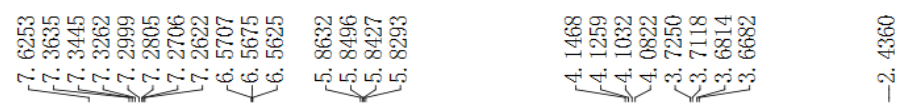

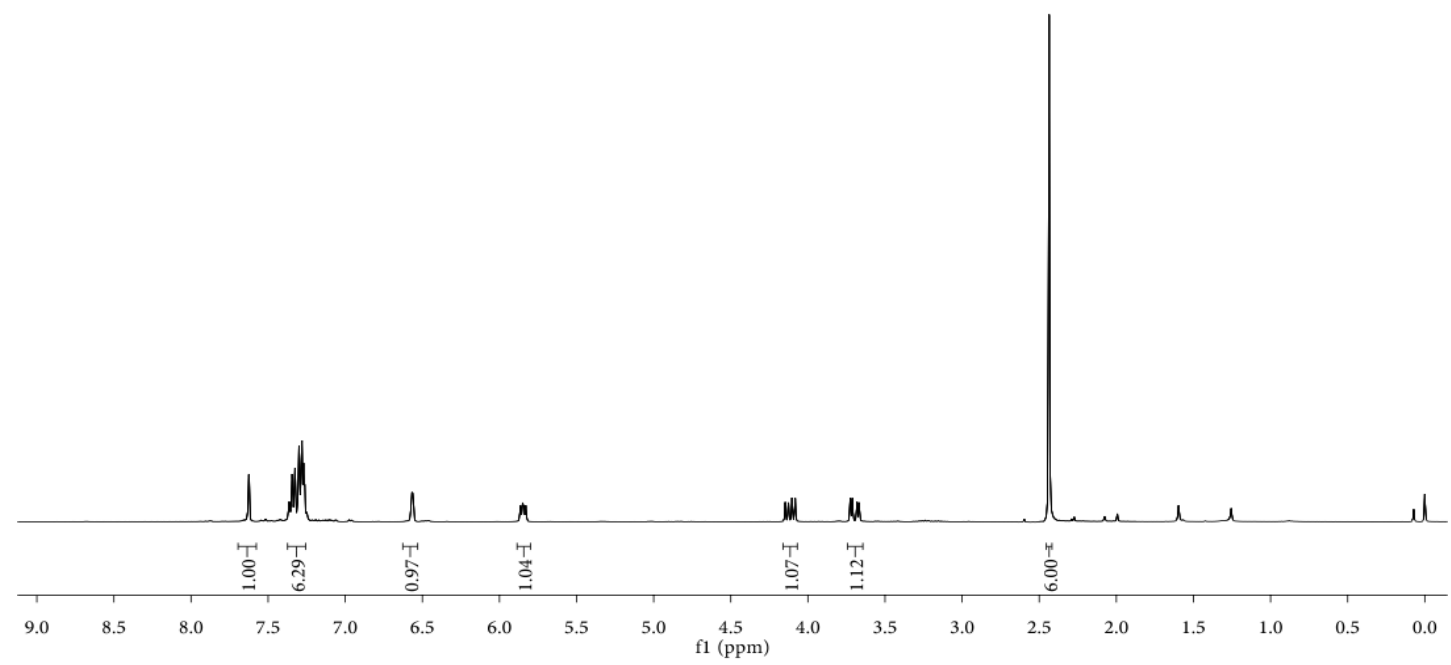


$N$-acetyl- $N$-(3-oxo-1-phenylbutyl)acetamide (10):<smiles>CC(=O)CC(c1ccccc1)N(C(C)=O)C(C)=O</smiles>

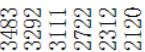

irivicio

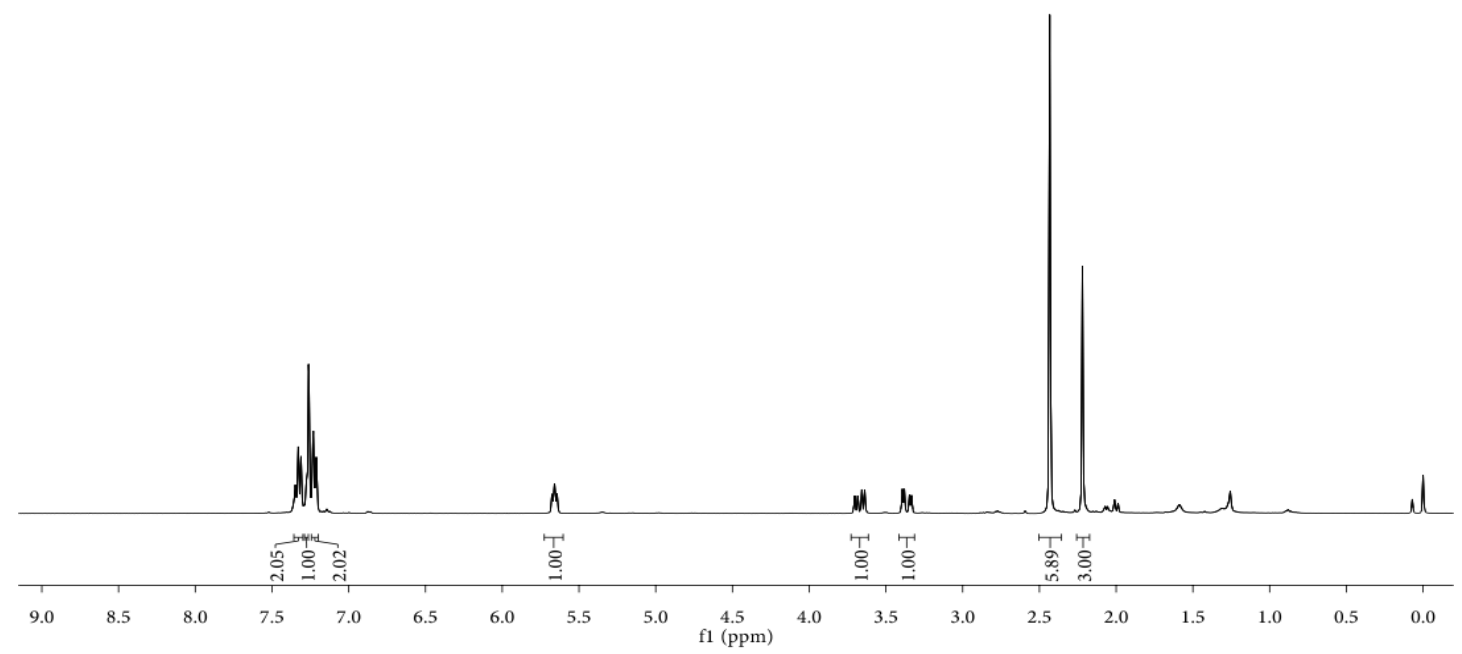

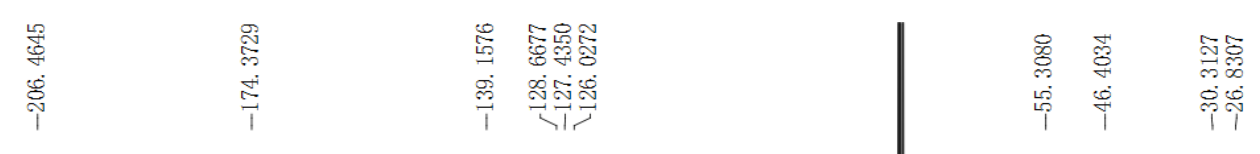

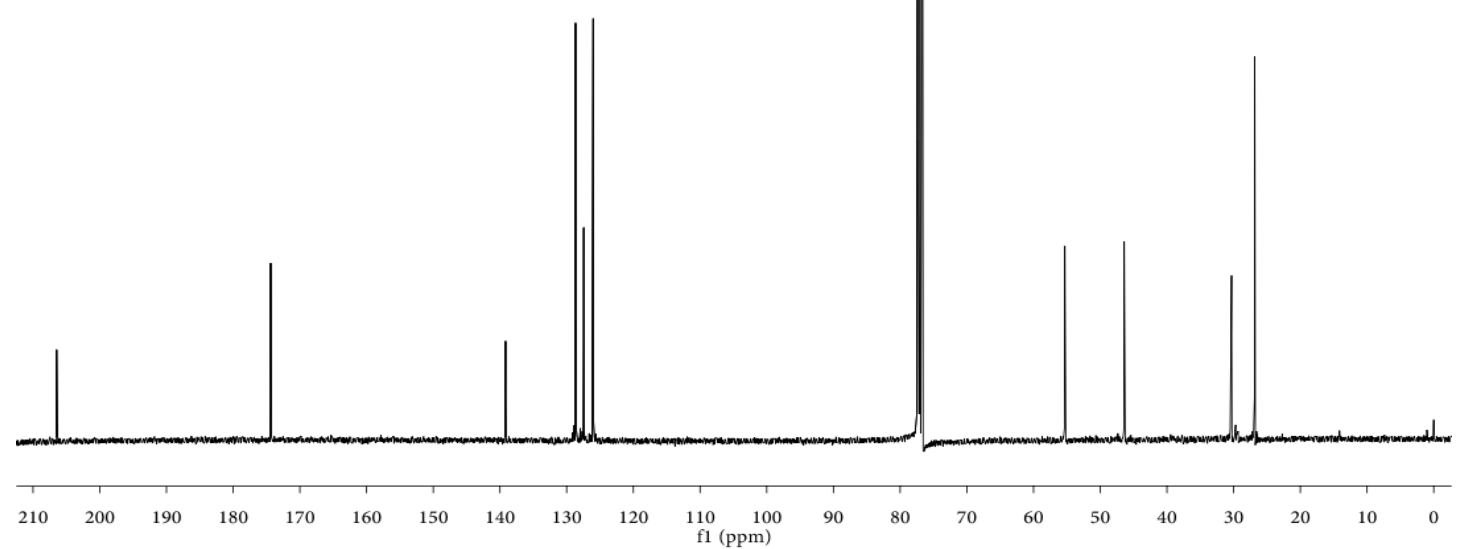


$N$-acetyl- $N$-(3-oxo-1-phenylpentyl)acetamide (11):

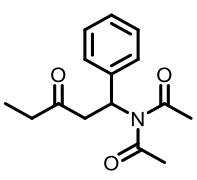

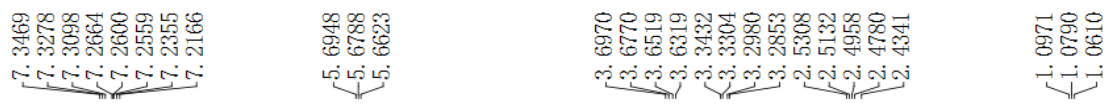

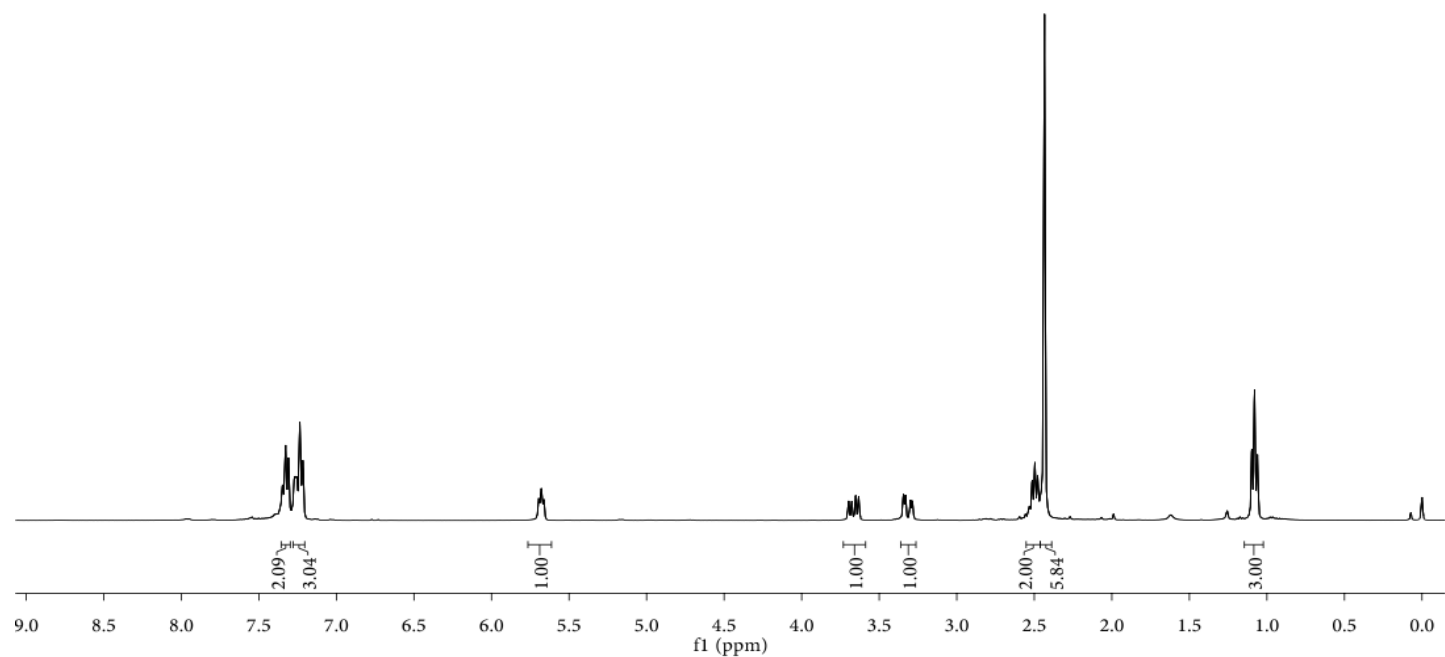

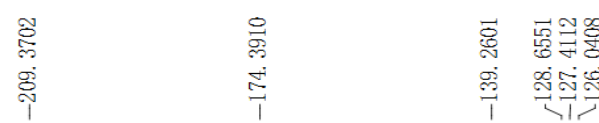

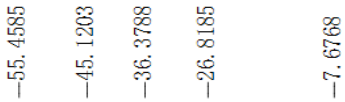

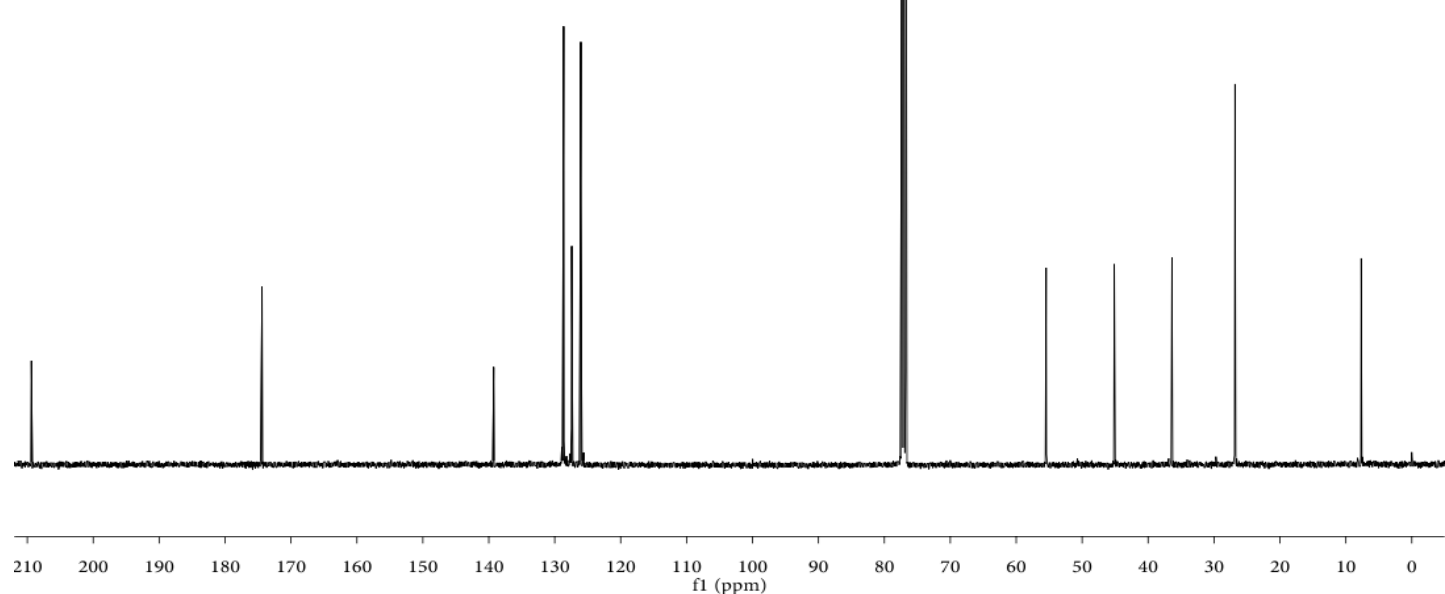


$N$-acetyl- $N$-(3-oxo-1-phenylheptyl)acetamide (12):

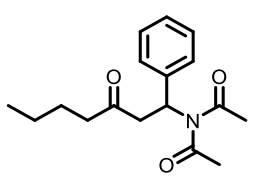

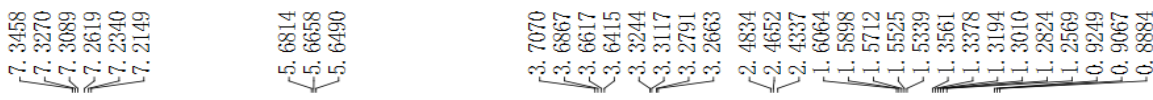

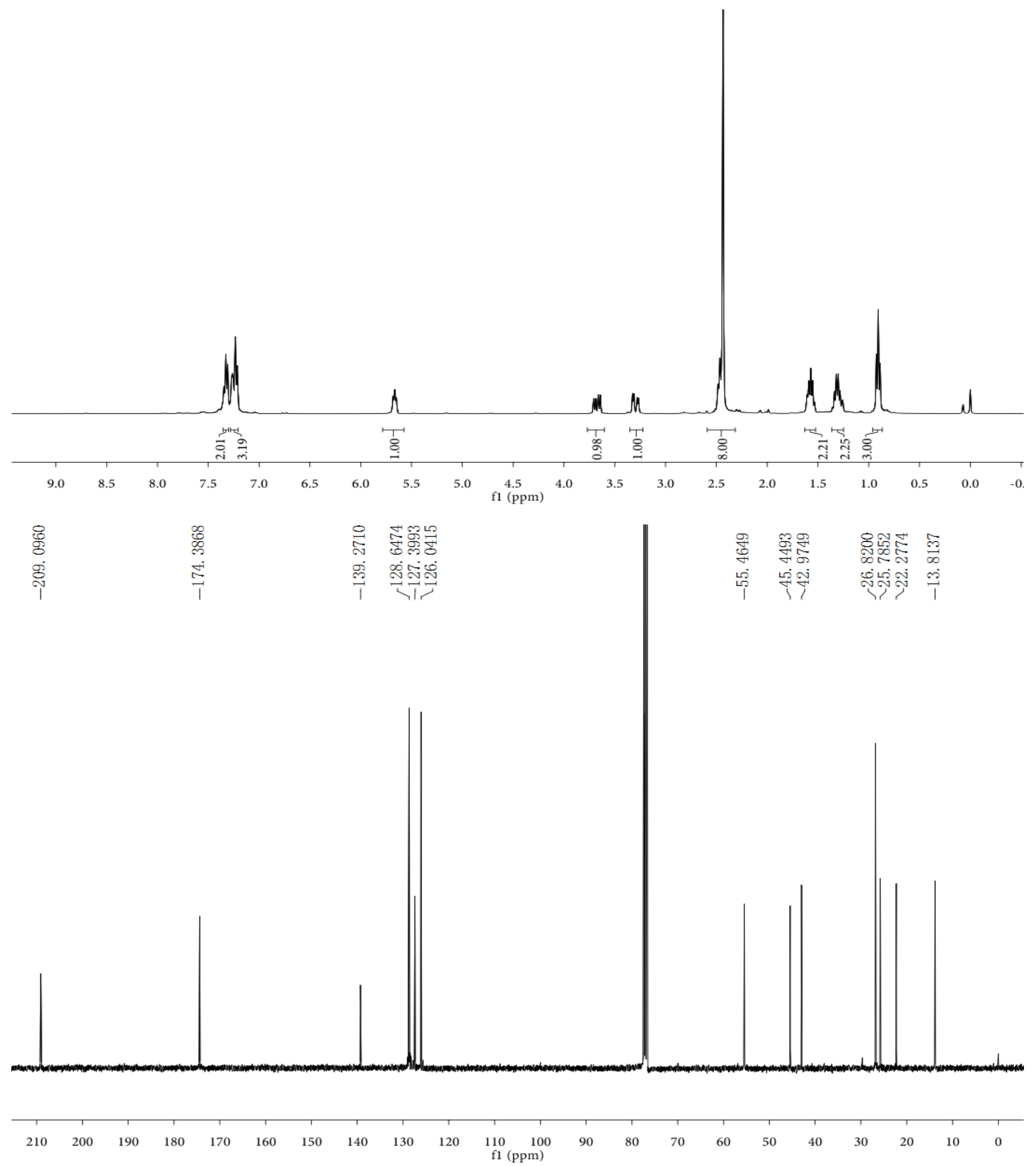


$N$-acetyl- $N$-(5-methyl-3-oxo-1-phenylhexyl)acetamide (13):

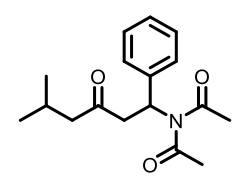

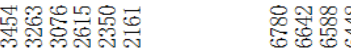

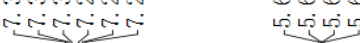

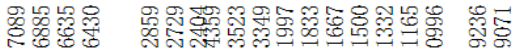

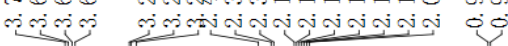

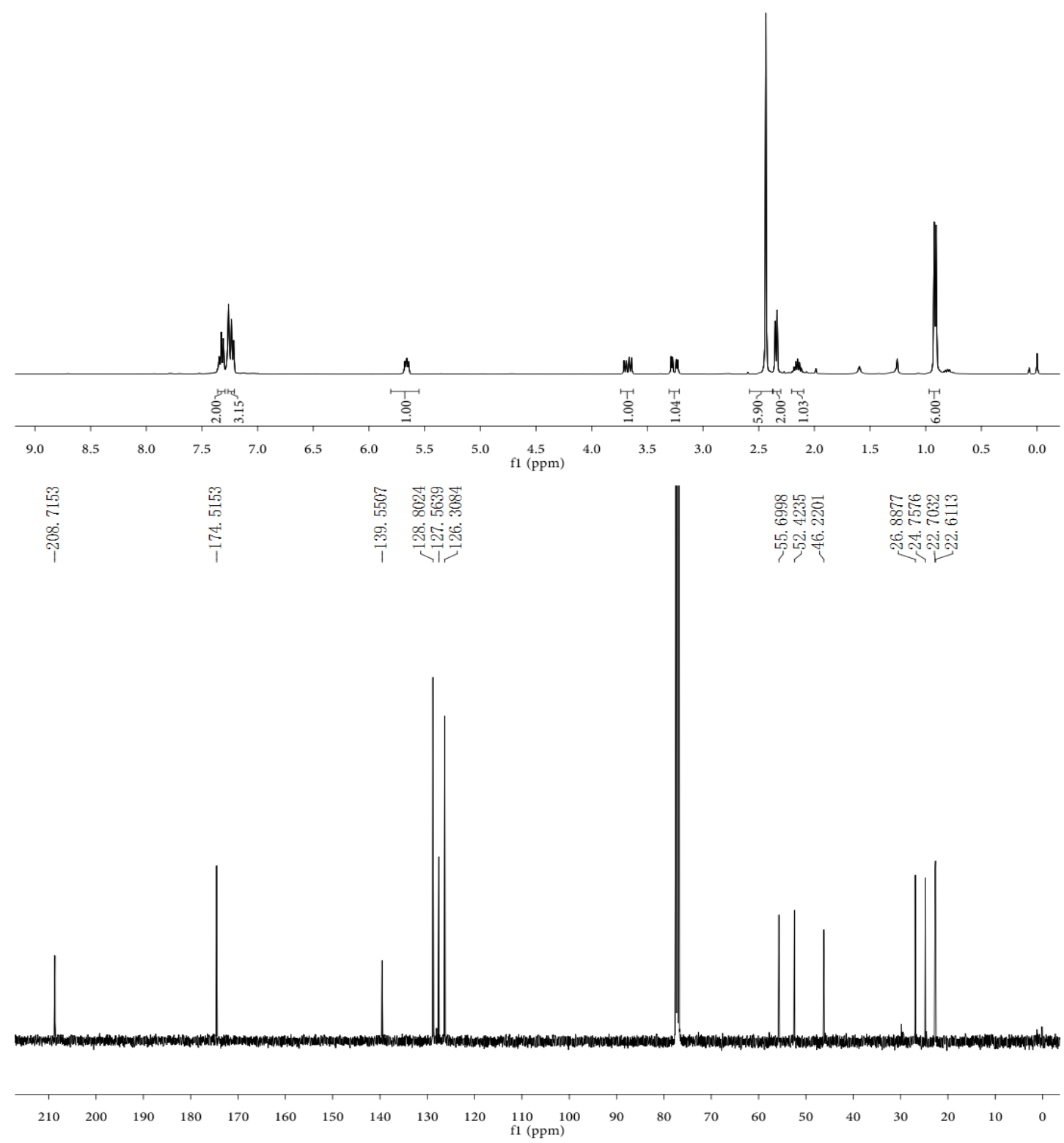


$N$-acetyl- $N$-(5-methyl-3-oxo-1-phenylheptyl)acetamide (14):

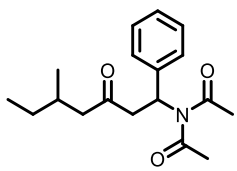

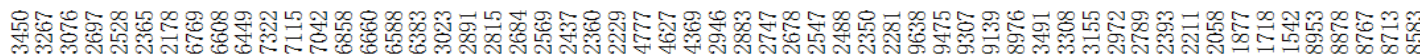

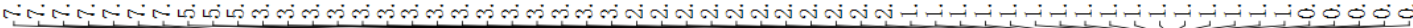

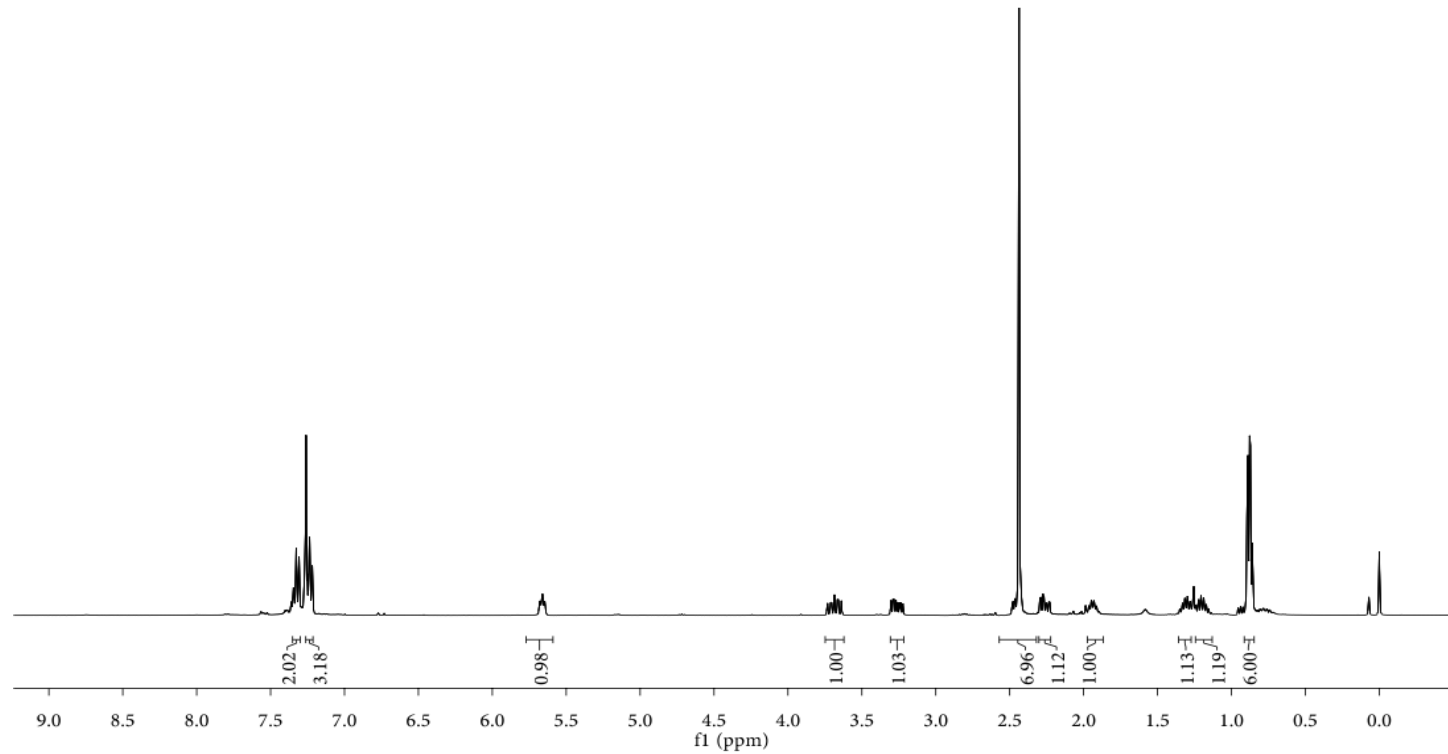

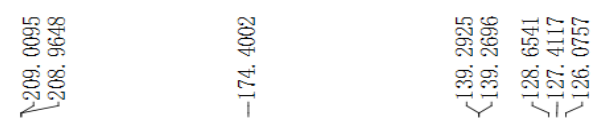

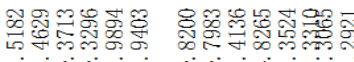

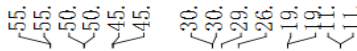

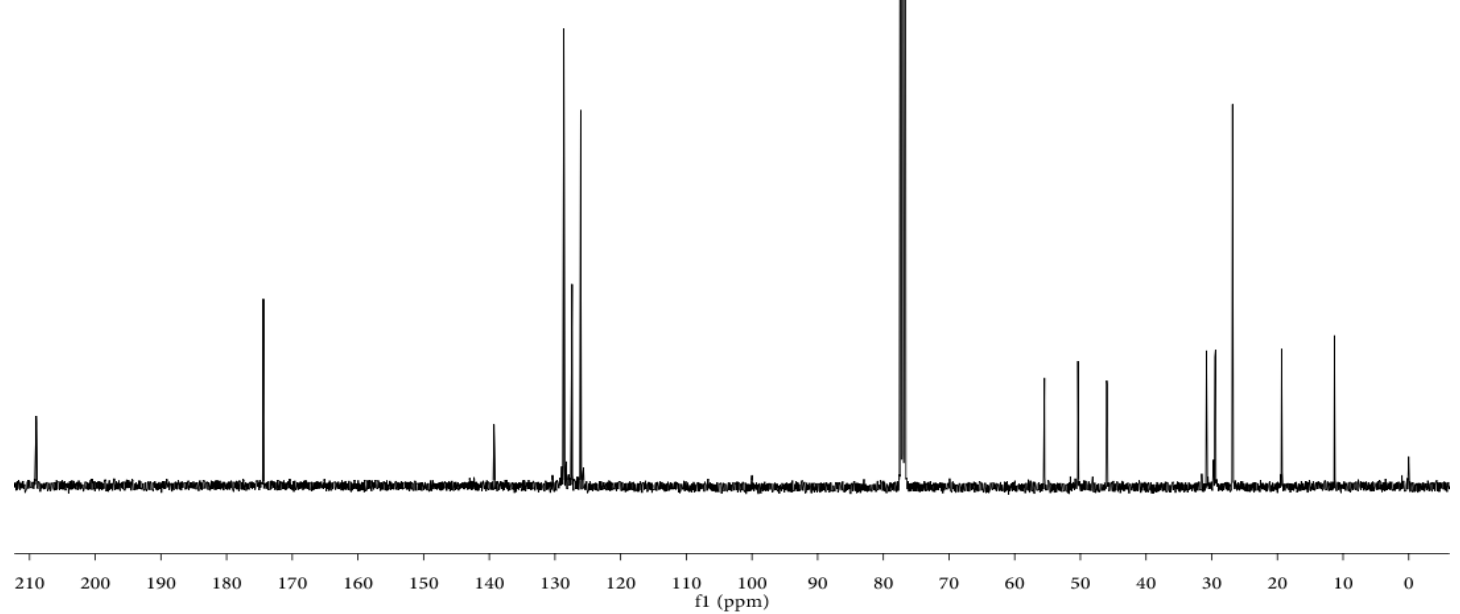


$N$-acetyl- $N$-(3-oxo-1,3-diphenylpropyl)propionamide (15):

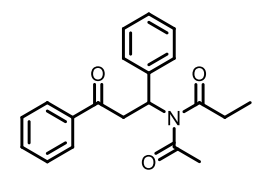

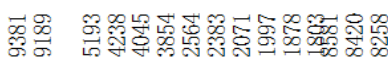

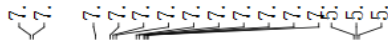

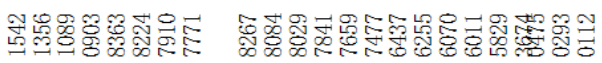

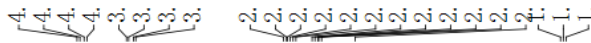

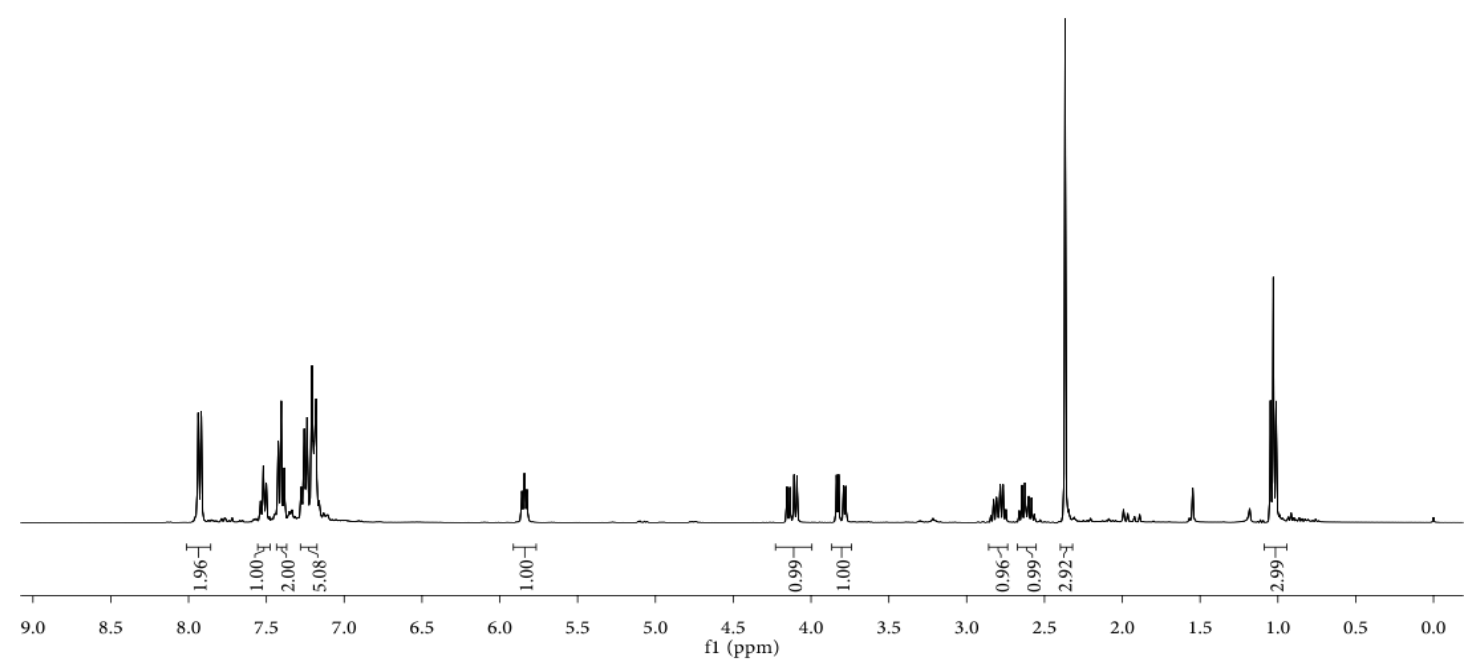

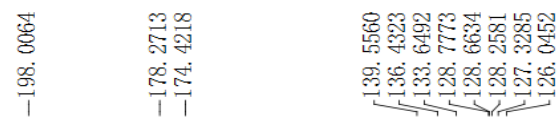

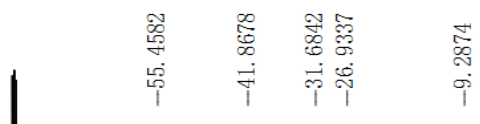

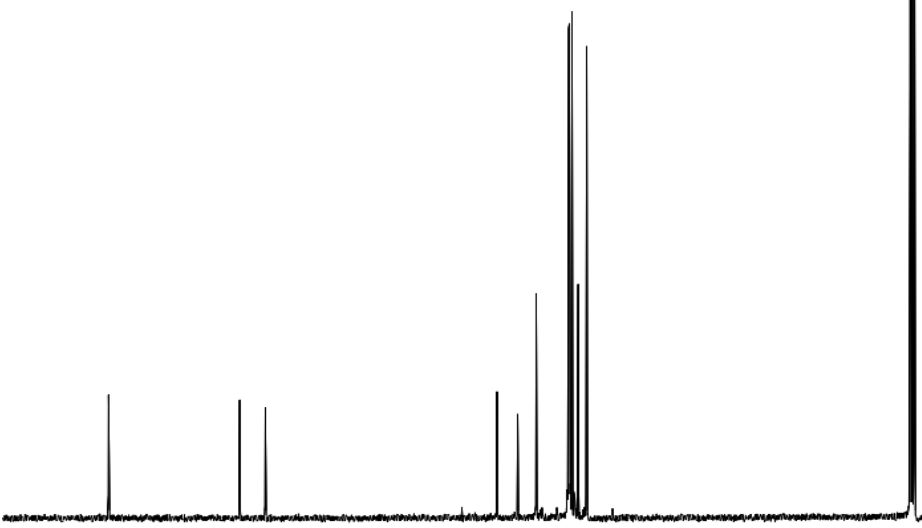

$\begin{array}{lllllllllll}210 & 200 & 190 & 180 & 170 & 160 & 150 & 140 & 130 & 120 & \begin{array}{l}110 \\ \mathrm{f} 1(\mathrm{ppm})\end{array}\end{array}$

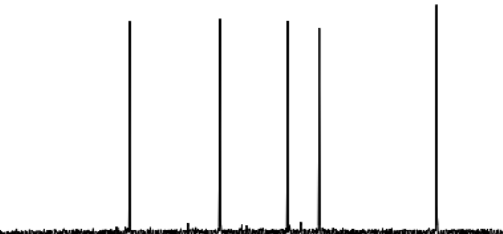


$N$-acetyl- $N$-(3-oxo-1,3-diphenylpropyl)hexanamide (16):

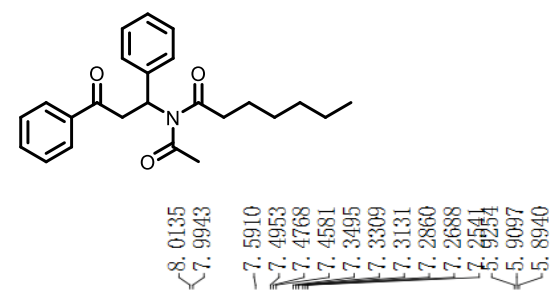

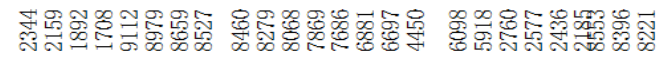

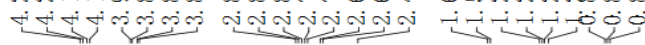
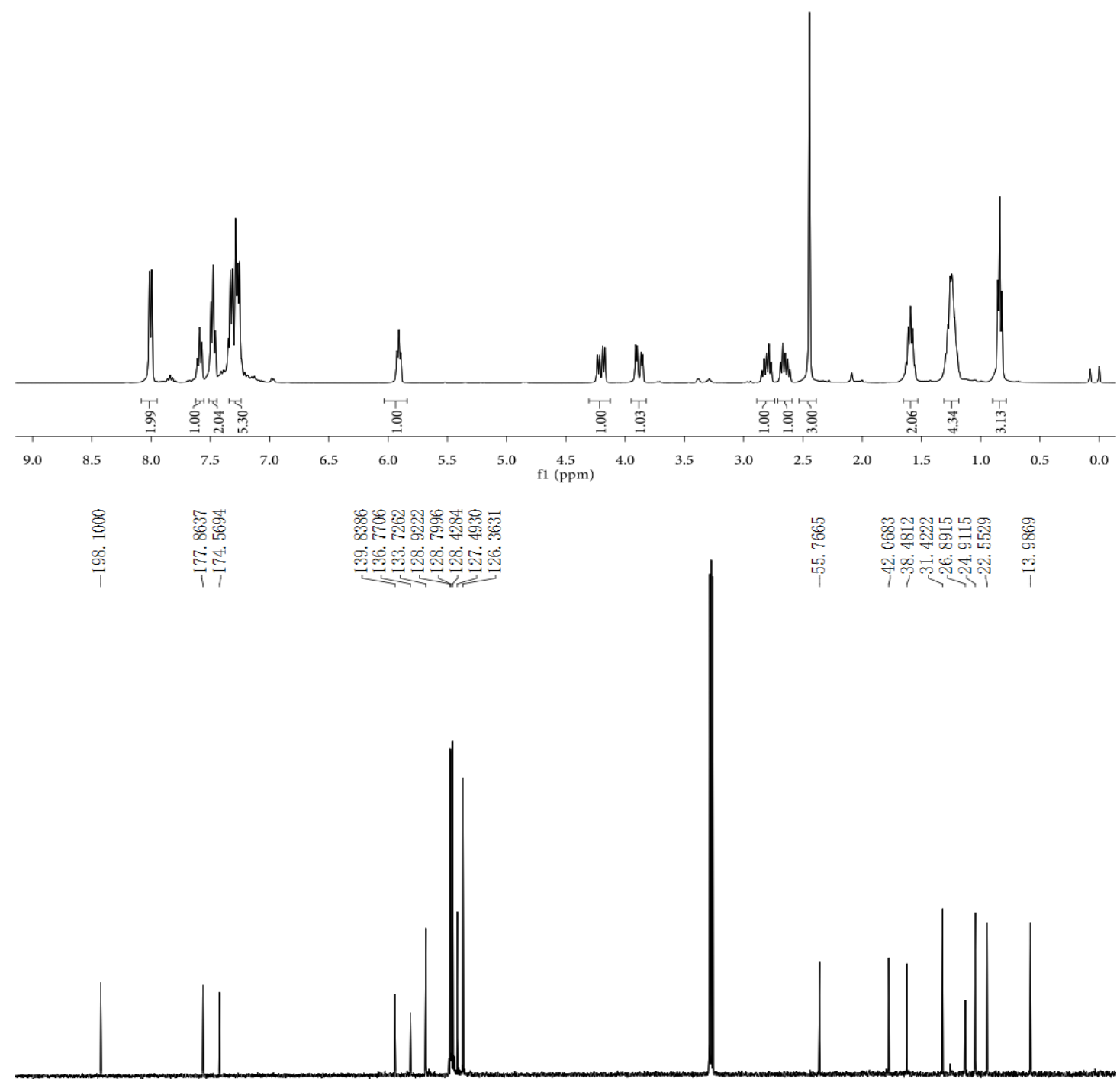

$\begin{array}{llllllllllllllllllllll}210 & 200 & 190 & 180 & 170 & 160 & 150 & 140 & 130 & 120 & \begin{array}{l}110 \\ \mathrm{f} 1(\mathrm{ppm})\end{array} & 90 & 80 & 70 & 60 & 50 & 40 & 30 & 20 & 10 & 0\end{array}$ 
$N$-acetyl- $N$-(3-oxo-1,3-diphenylpropyl)tridecanamide (17):

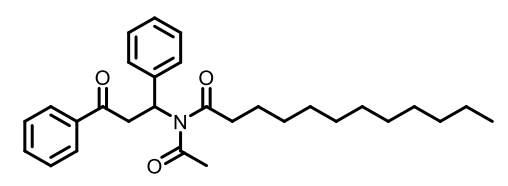

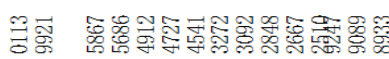

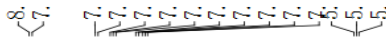
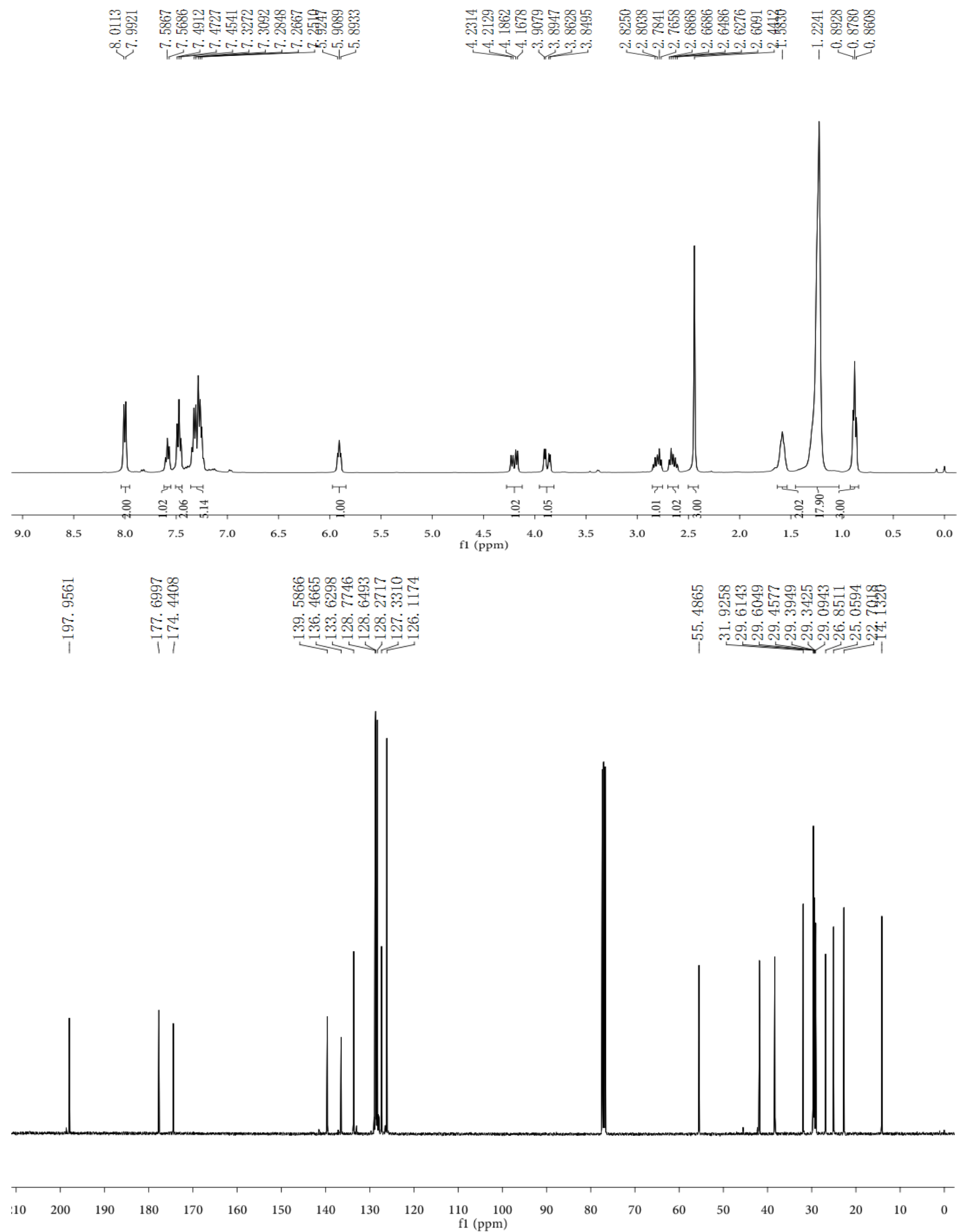
$N$-acetyl- $N$-(3-oxo-1,3-diphenylpropyl)tridecanamide (18):
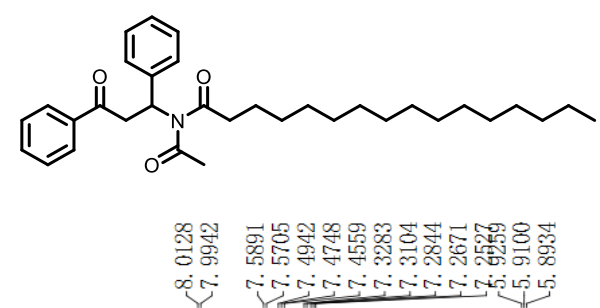

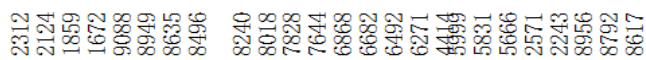

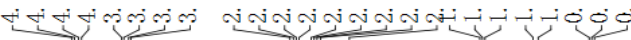
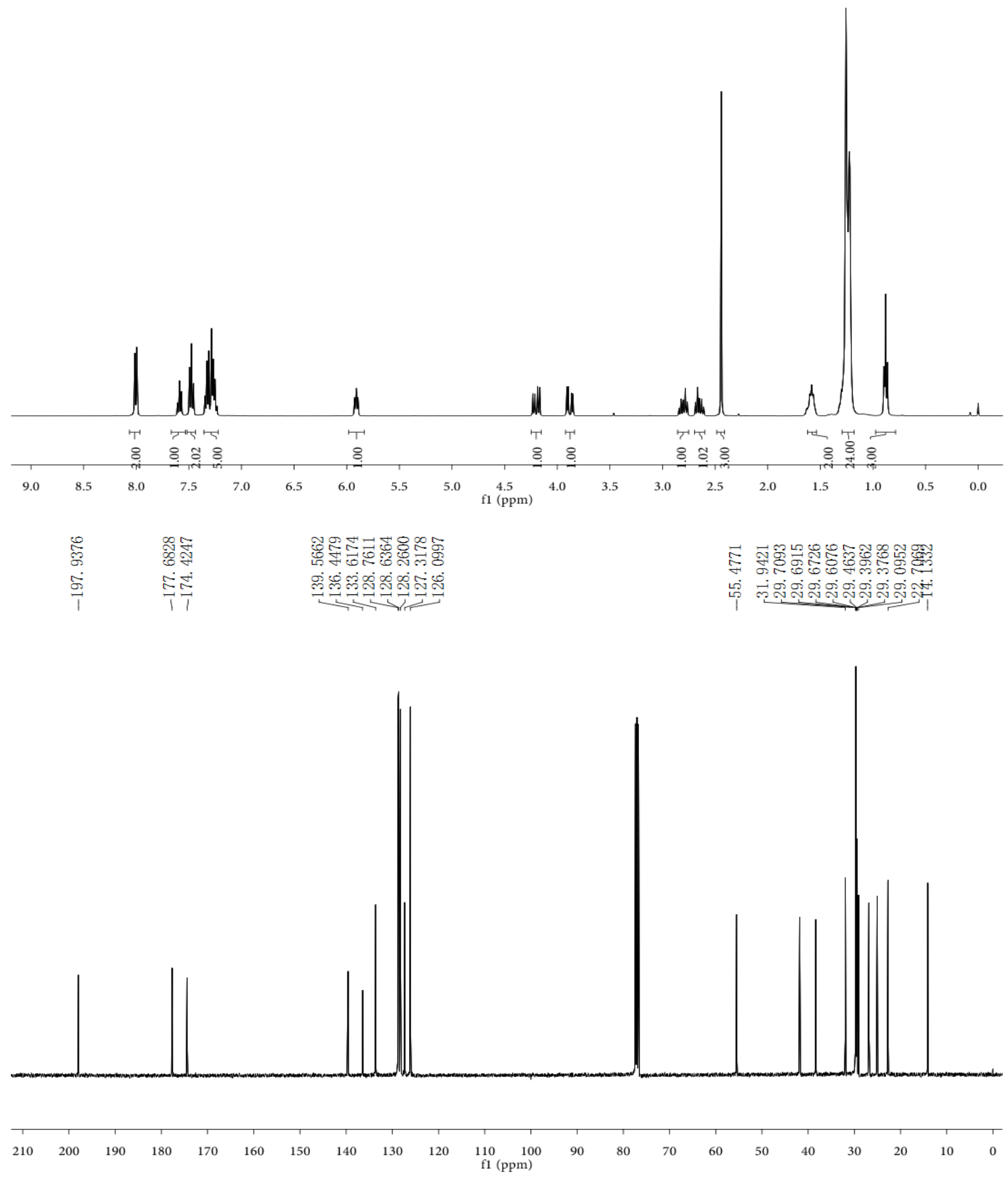
Ethyl 4-oxo-4-( $N$-(3-oxo-1,3-diphenylpropyl)acetamido)butanoate (19):
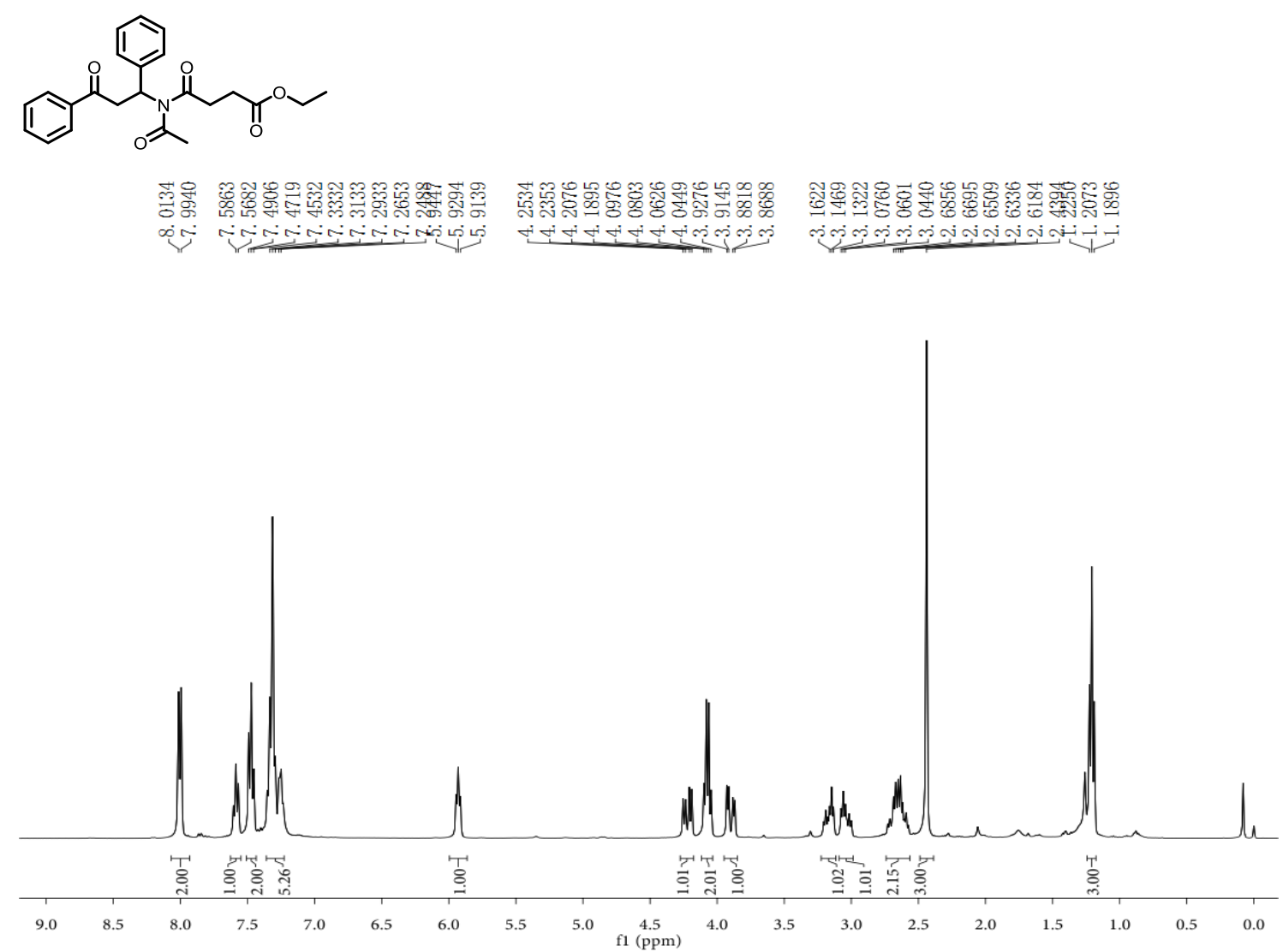

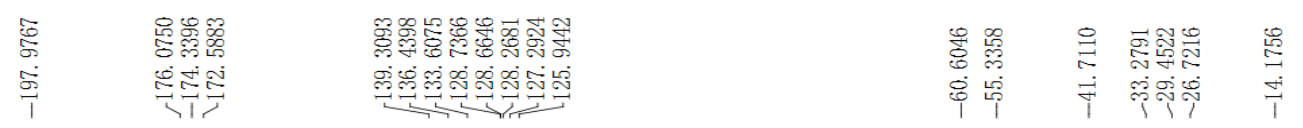

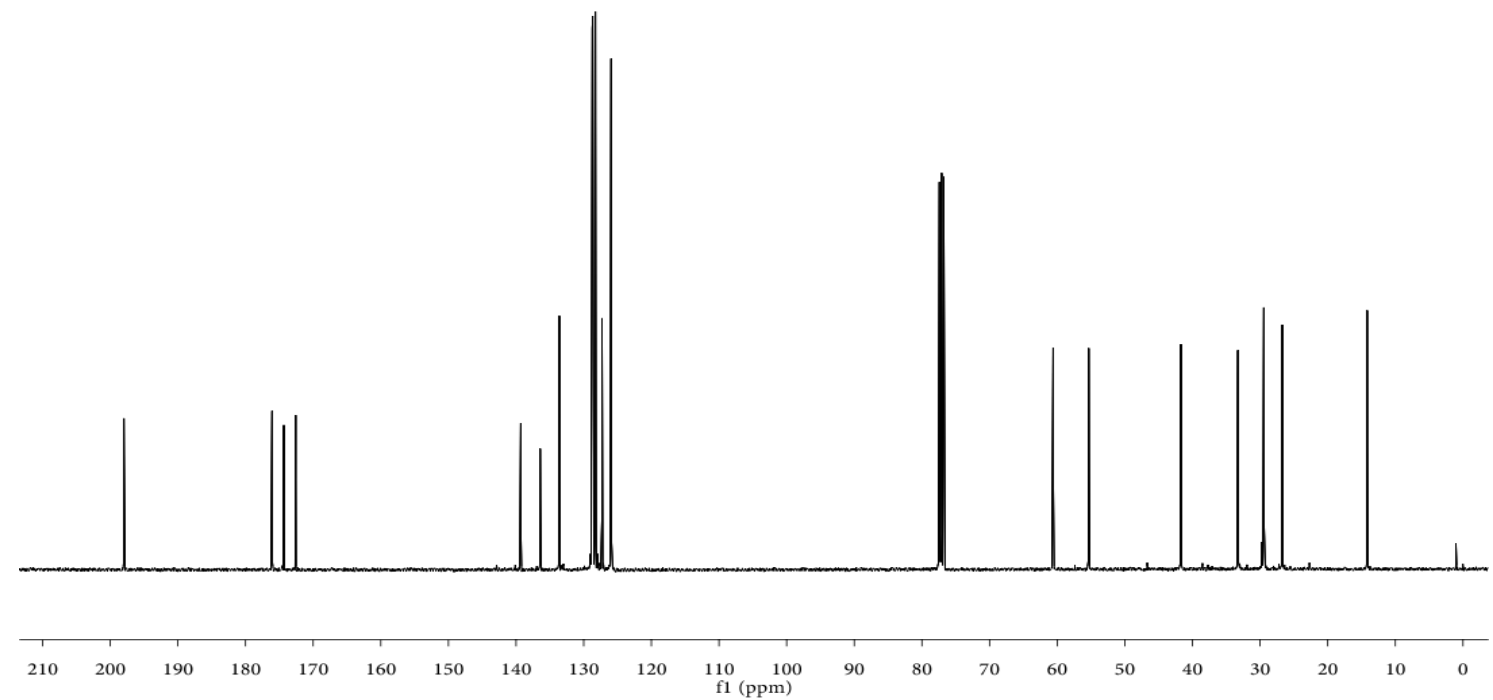


$N$-acetyl- $N$-(3-oxo-1,3-diphenylpropyl)cyclopropanecarboxamide (20):<smiles>CC(=O)N(C(=O)C1CC1)C(CC(=O)c1ccccc1)c1ccccc1</smiles>

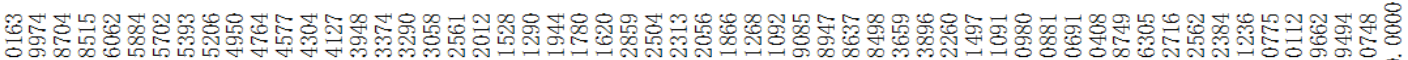

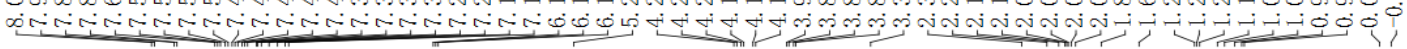

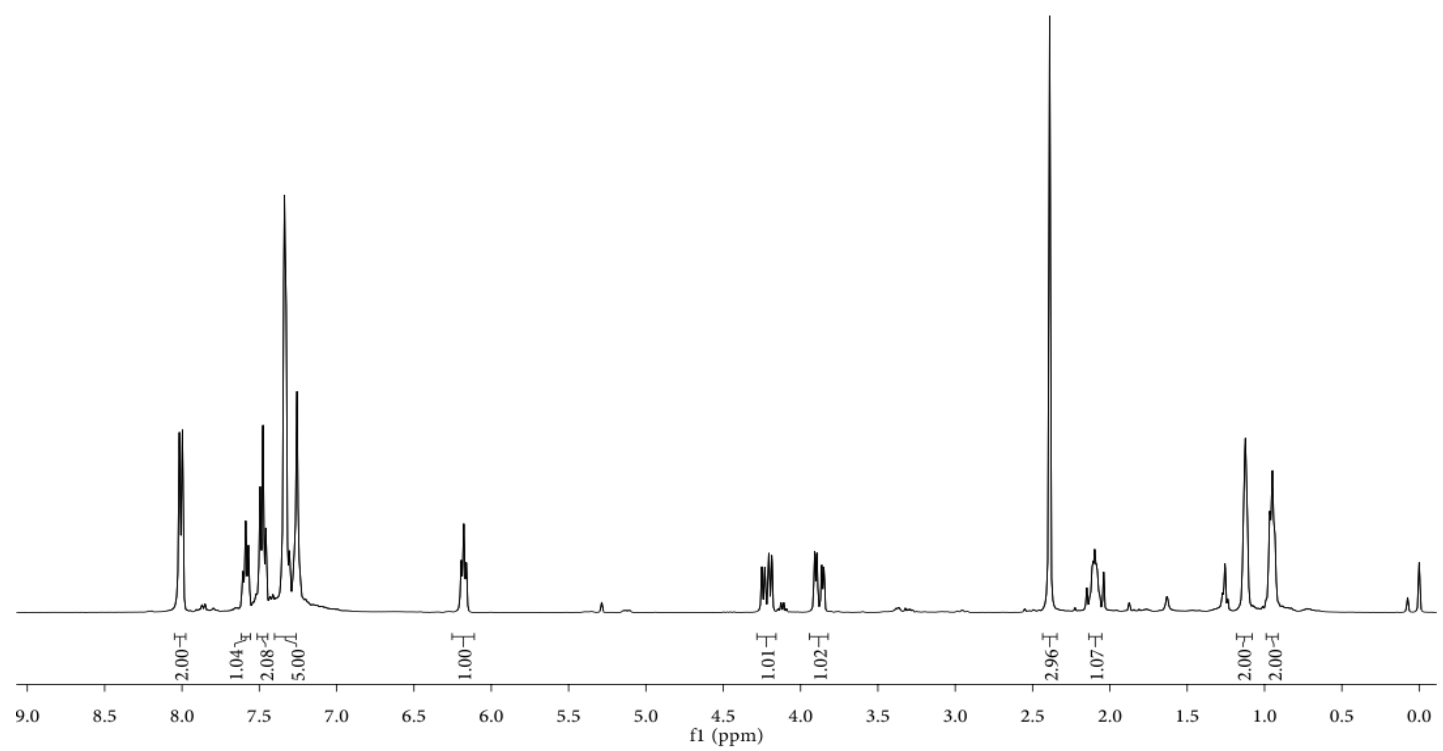

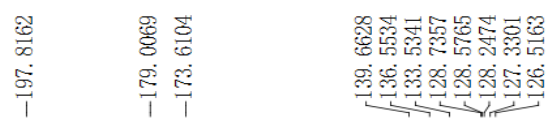

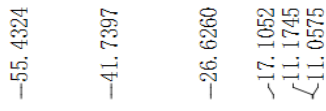

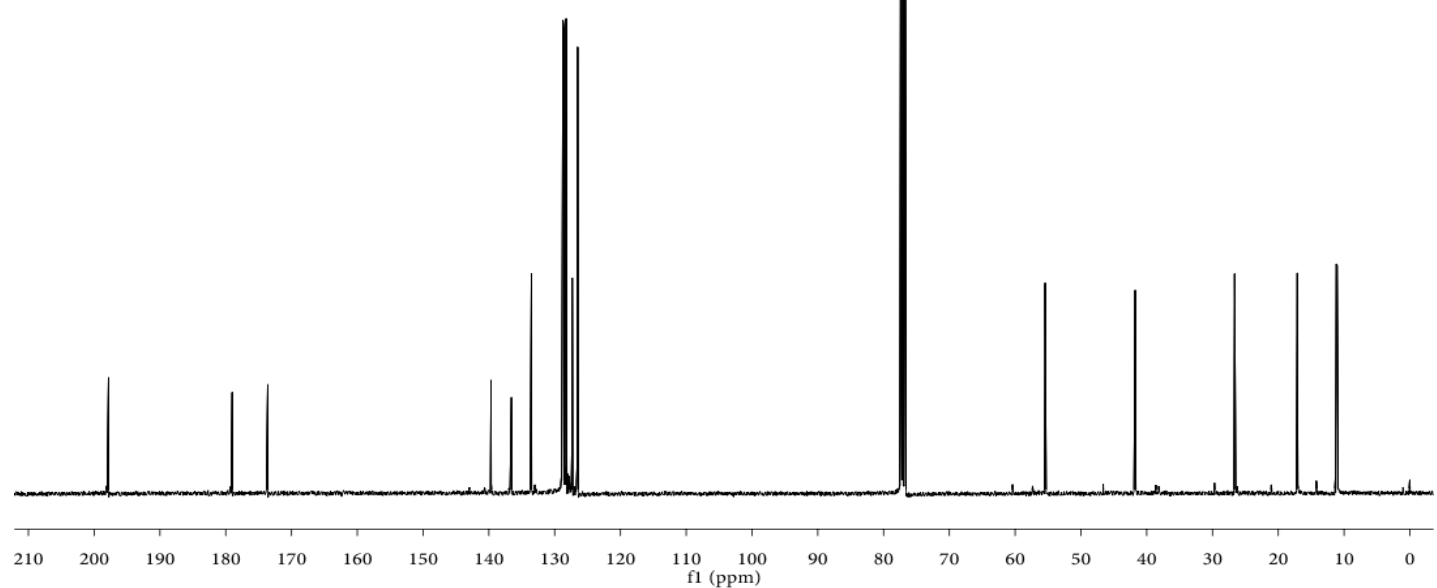


$N$-acetyl- $N$-(3-oxo-1,3-diphenylpropyl)cyclobutanecarboxamide (21):

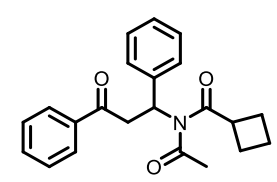

퐁녕

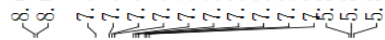

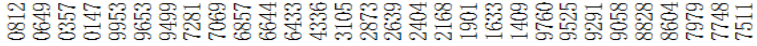

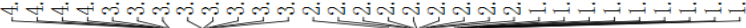

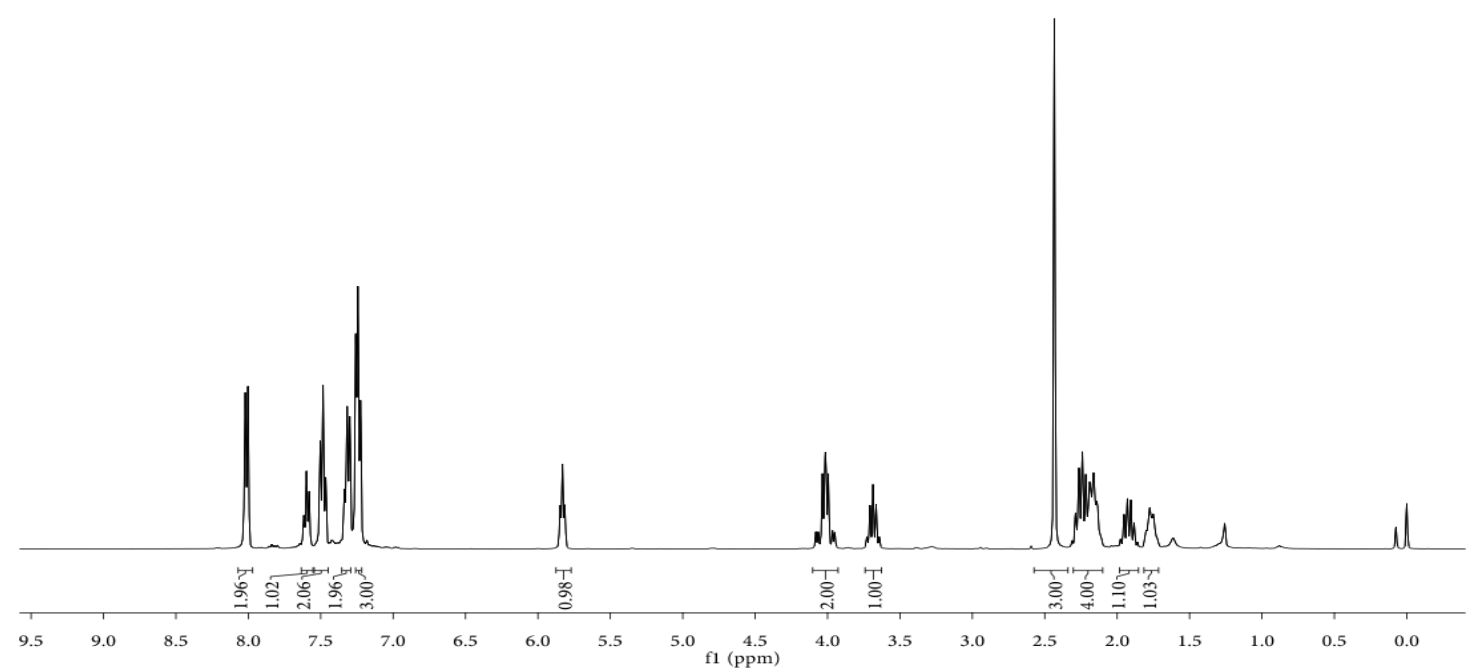

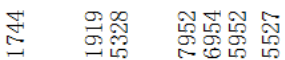

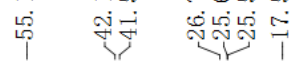

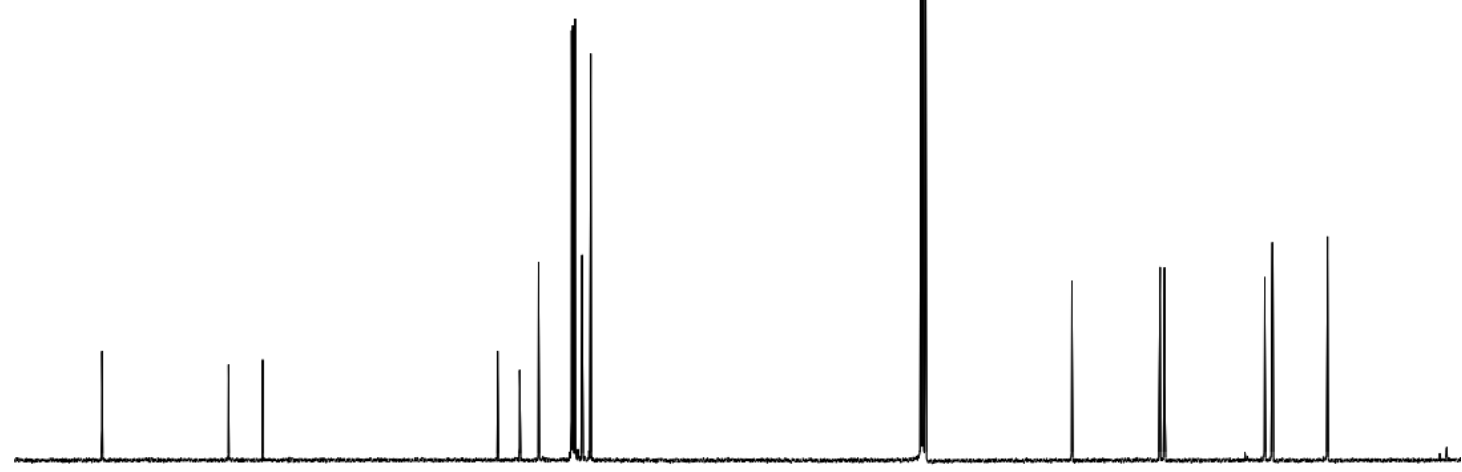

$\begin{array}{lllllllllll}10 & 200 & 190 & 180 & 170 & 160 & 150 & 140 & 130 & 120 & \begin{array}{c}110 \\ \mathrm{f} 1(\mathrm{ppm})\end{array}\end{array}$

$\begin{array}{llllllllll}90 & 80 & 70 & 60 & 50 & 40 & 30 & 20 & 10 & 0\end{array}$ 
$N$-acetyl- $N$-(3-oxo-1,3-diphenylpropyl)cyclopentanecarboxamide (22):
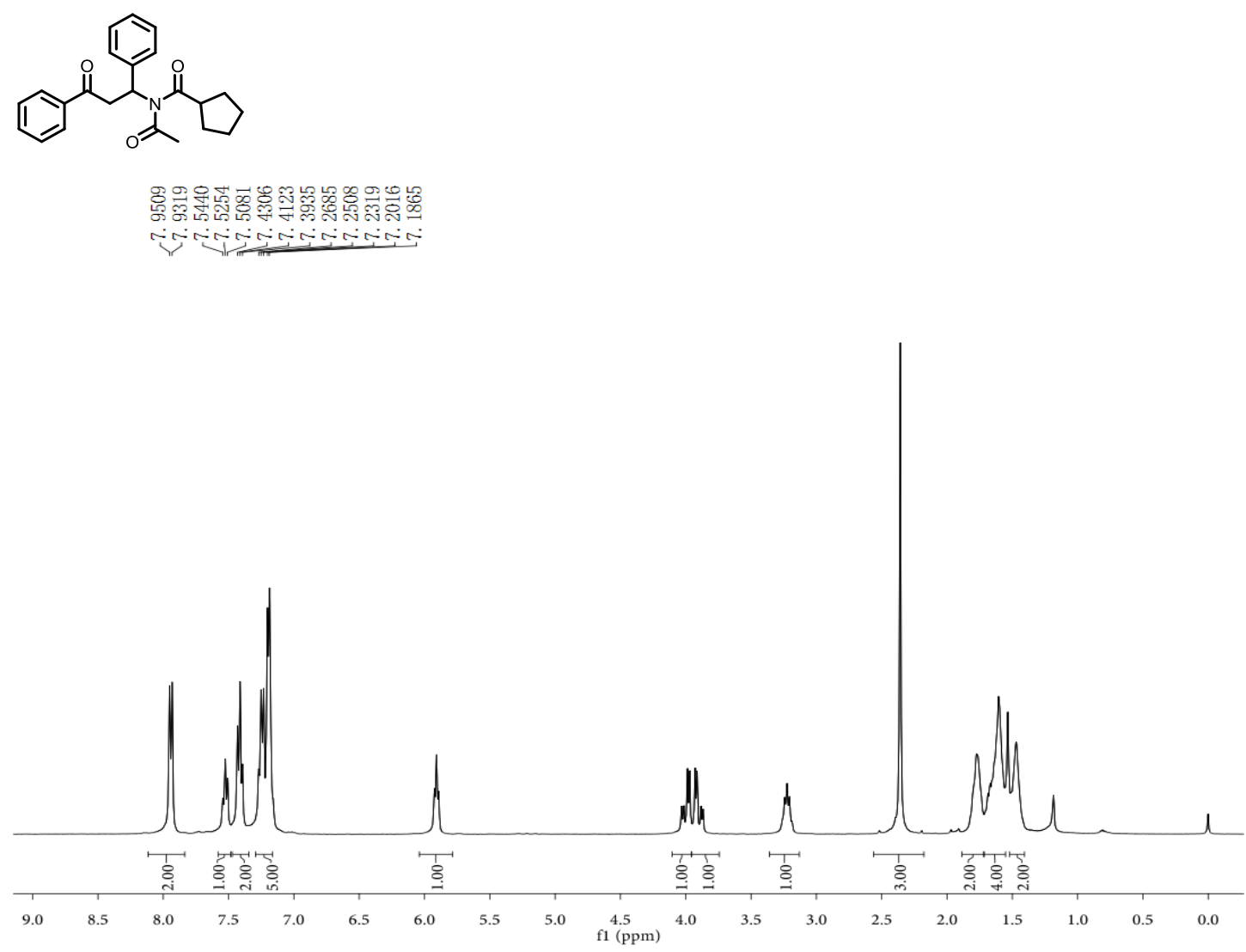

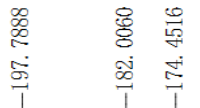

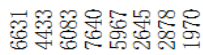

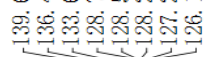

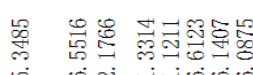

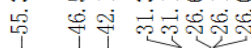

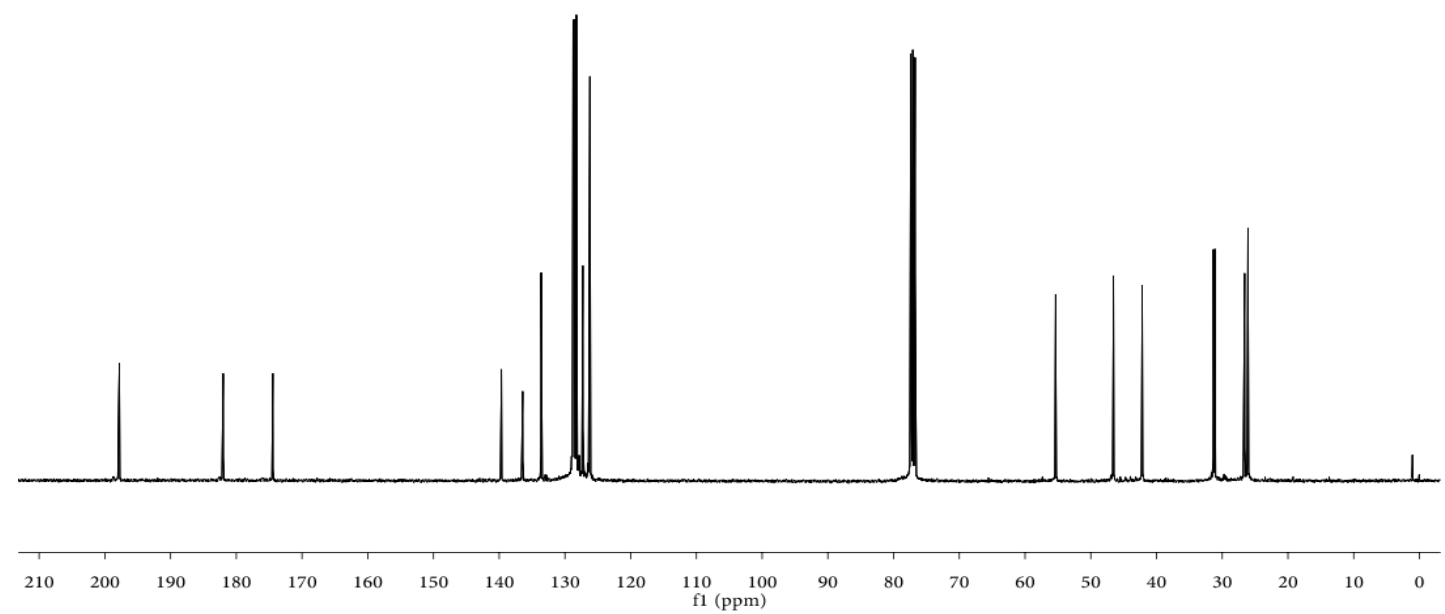


$N$-acetyl- $N$-(3-oxo-1,3-diphenylpropyl)-2-phenylacetamide (23):<smiles>CC(=O)N(Cc1ccccc1)C(=O)Cc1ccccc1</smiles>

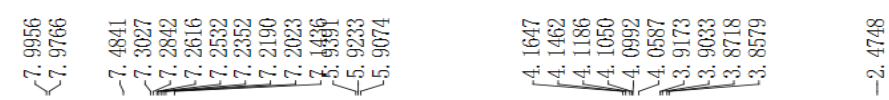

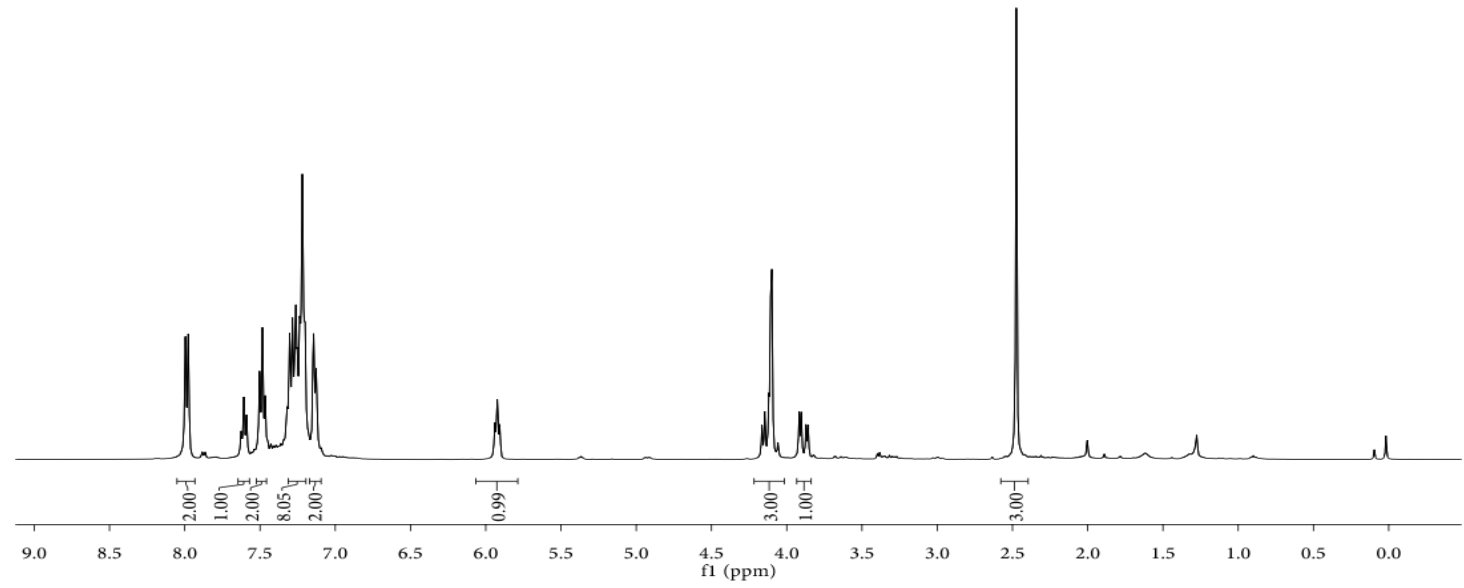

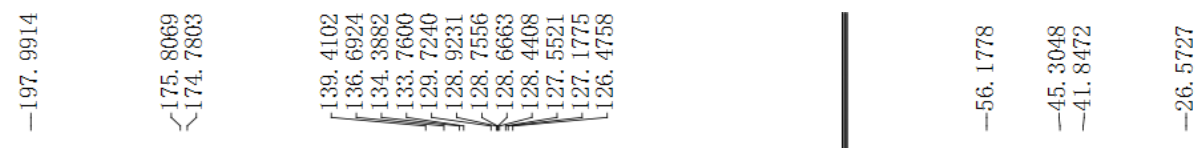

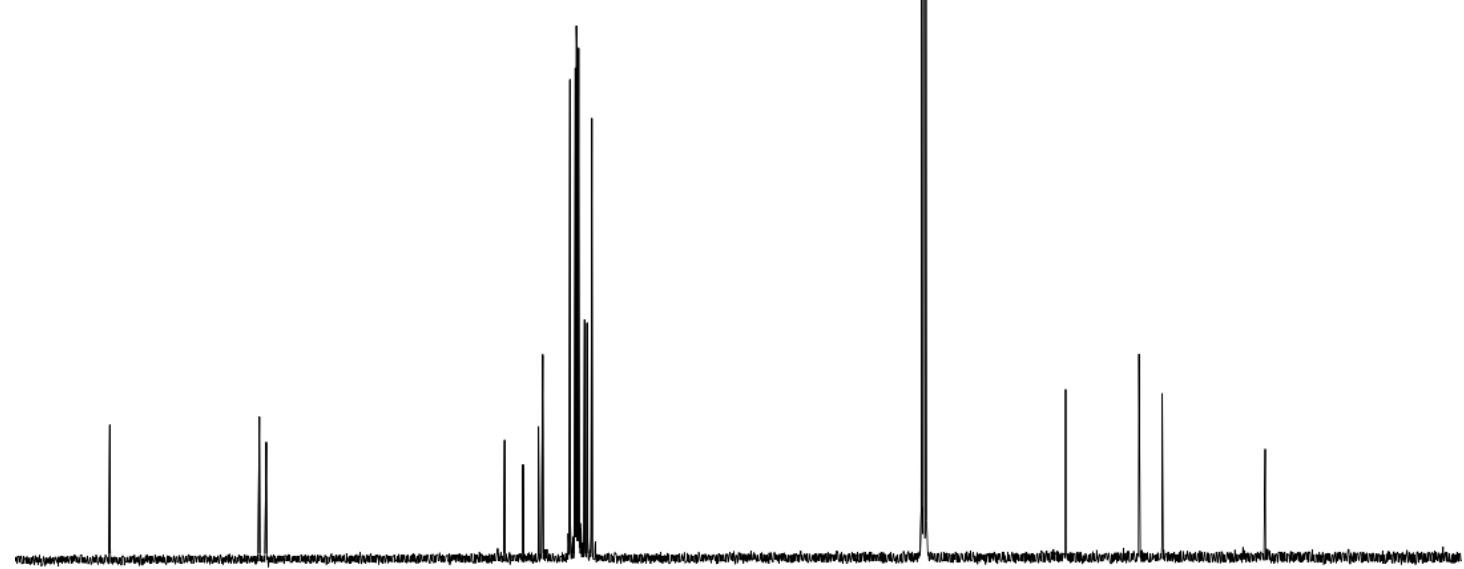

$\begin{array}{lllllllllllllllllllll}210 & 200 & 190 & 180 & 170 & 160 & 150 & 140 & 130 & 120 & \begin{array}{c}110 \\ \mathrm{f} 1(\mathrm{ppm})\end{array} & 90 & 80 & 70 & 60 & 50 & 40 & 30 & 20 & 10 & 0\end{array}$ 
$N$-acetyl- $N$-(3-oxo-1,3-diphenylpropyl)benzamide (24):

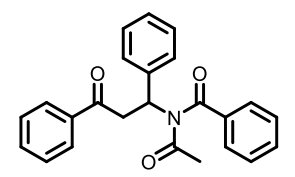

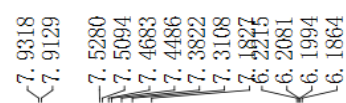

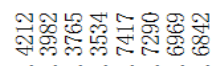

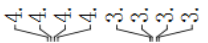

$\underset{\substack{\mathrm{d} \\ i}}{\stackrel{\vec{i}}{+}}$

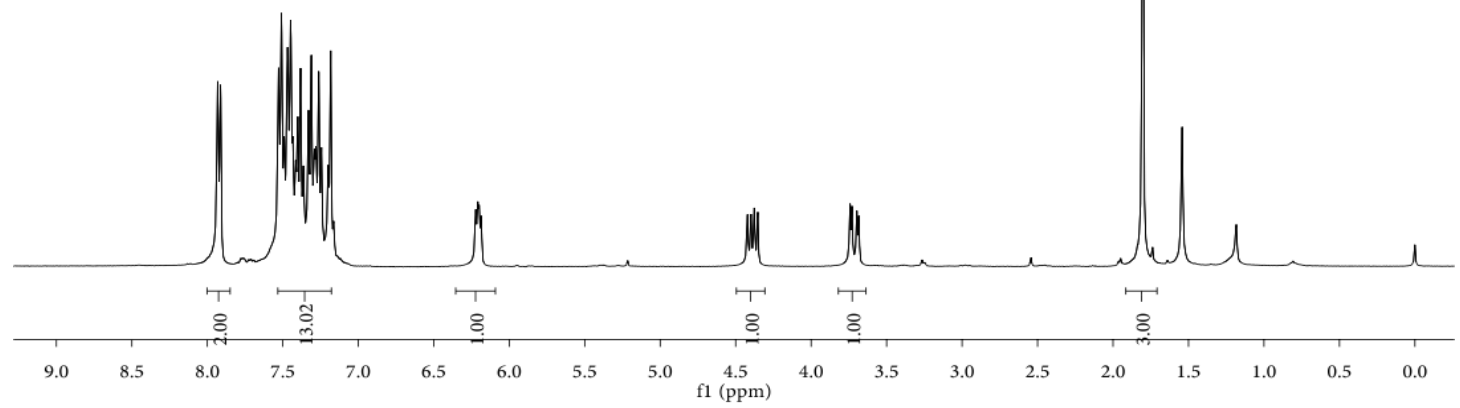

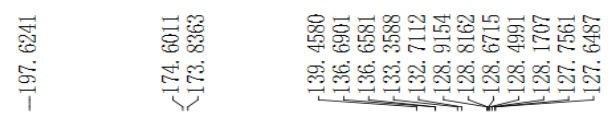

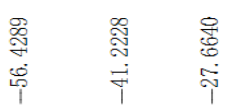

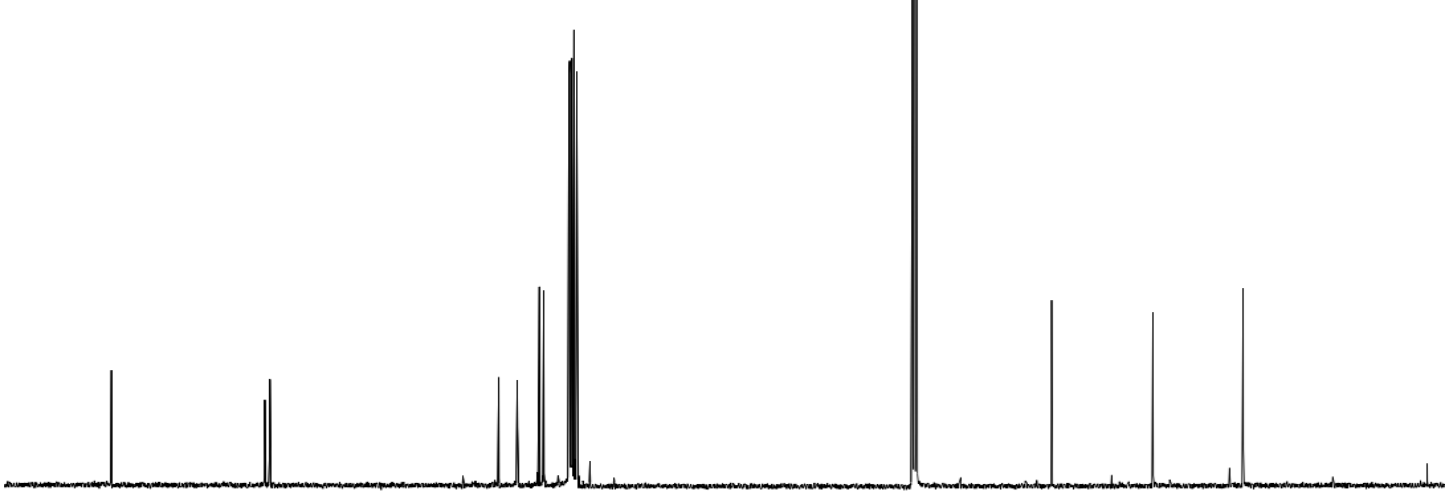

$\begin{array}{llllllllll}200 & 190 & 180 & 170 & 160 & 150 & 140 & 130 & 120 & \begin{array}{l}110 \\ \mathrm{f} 1(\mathrm{ppm})\end{array}\end{array}$

80

60

50

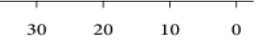


$N$-acetyl- $N$-(3-oxo-1,3-diphenylpropyl)isonicotinamide (25):

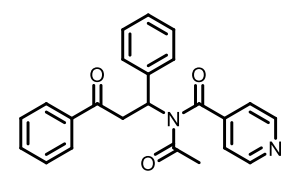

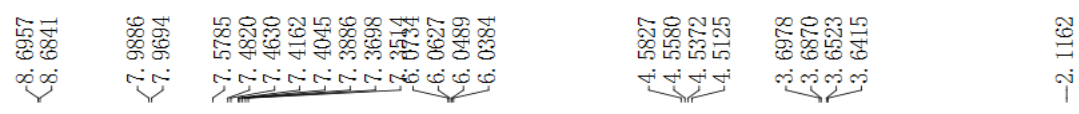
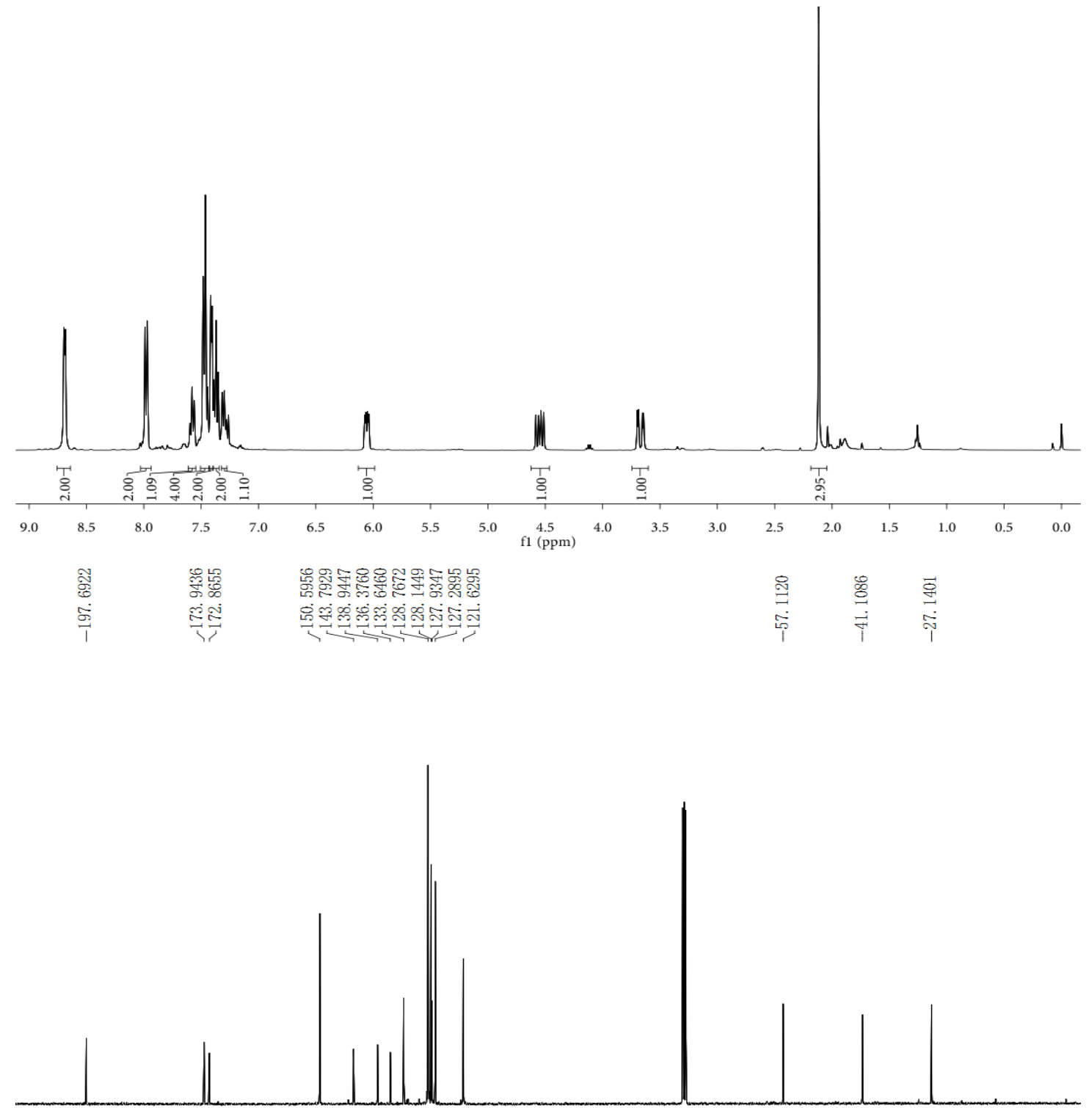

$\begin{array}{lllllllllll}210 & 200 & 190 & 180 & 170 & 160 & 150 & 140 & 130 & 120 & \begin{array}{c}110 \\ \mathrm{f} 1(\mathrm{ppm})\end{array}\end{array}$ 
$N$-acetyl- $N$-(3-oxo-1,3-diphenylpropyl)-2-naphthamide (26):

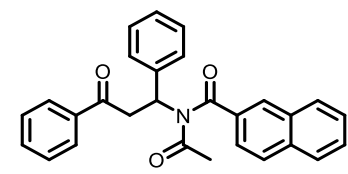

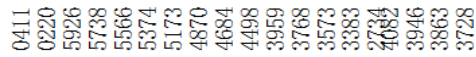

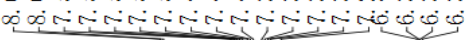

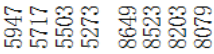

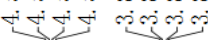

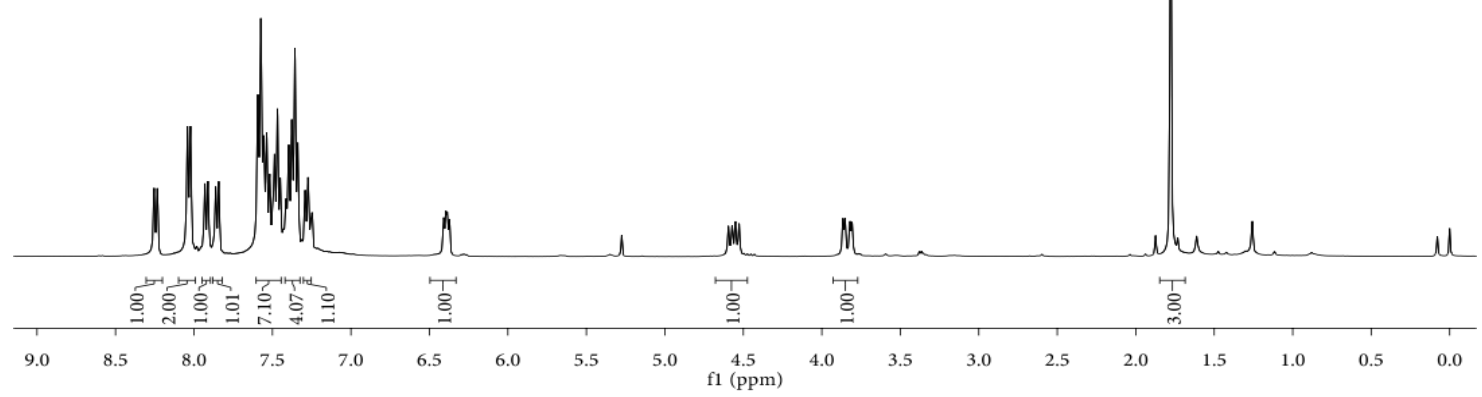

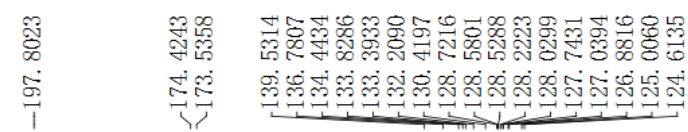

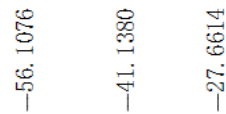

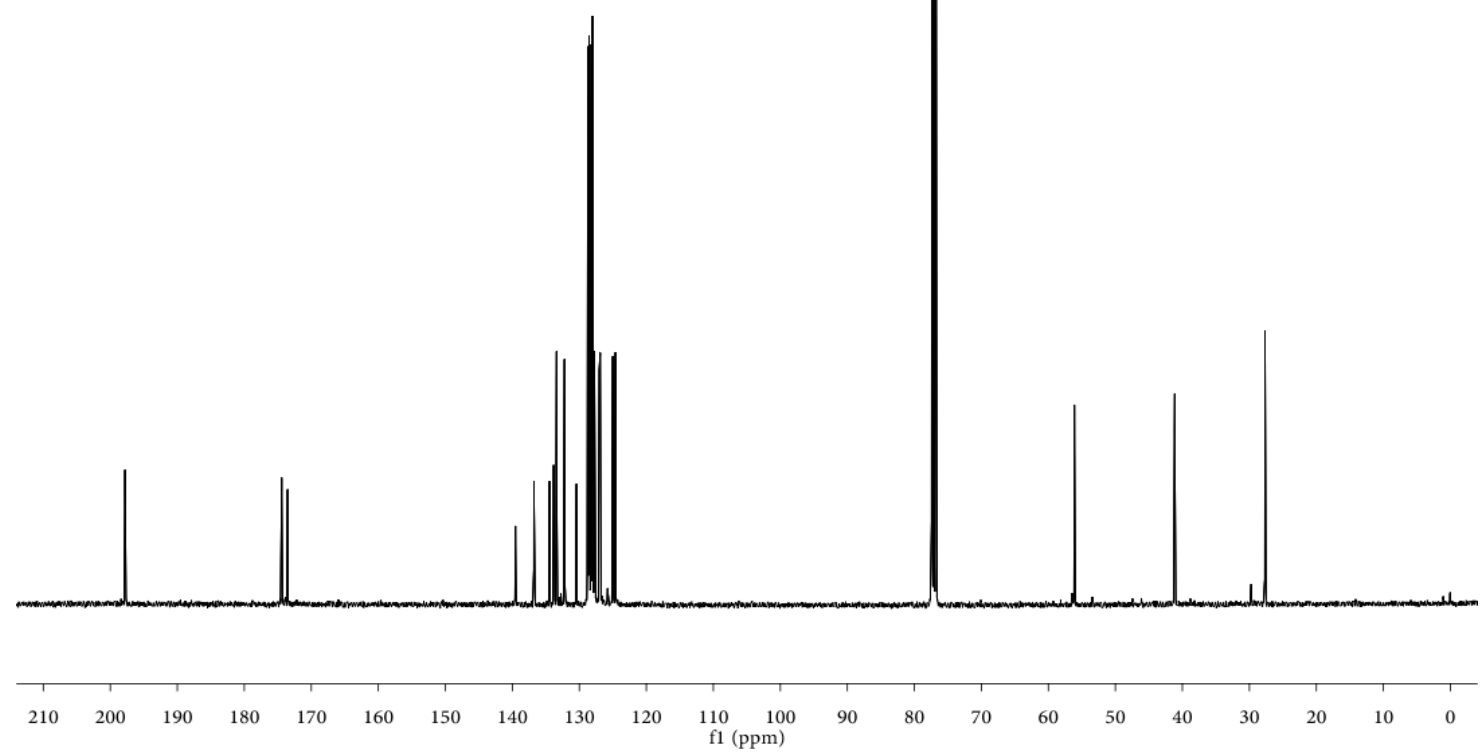


$N$-acetyl- $N$-(3-oxo-3-phenyl-1-(o-tolyl)propyl)acetamide (27):

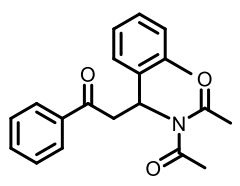

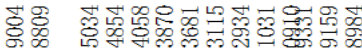

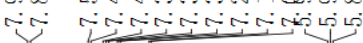

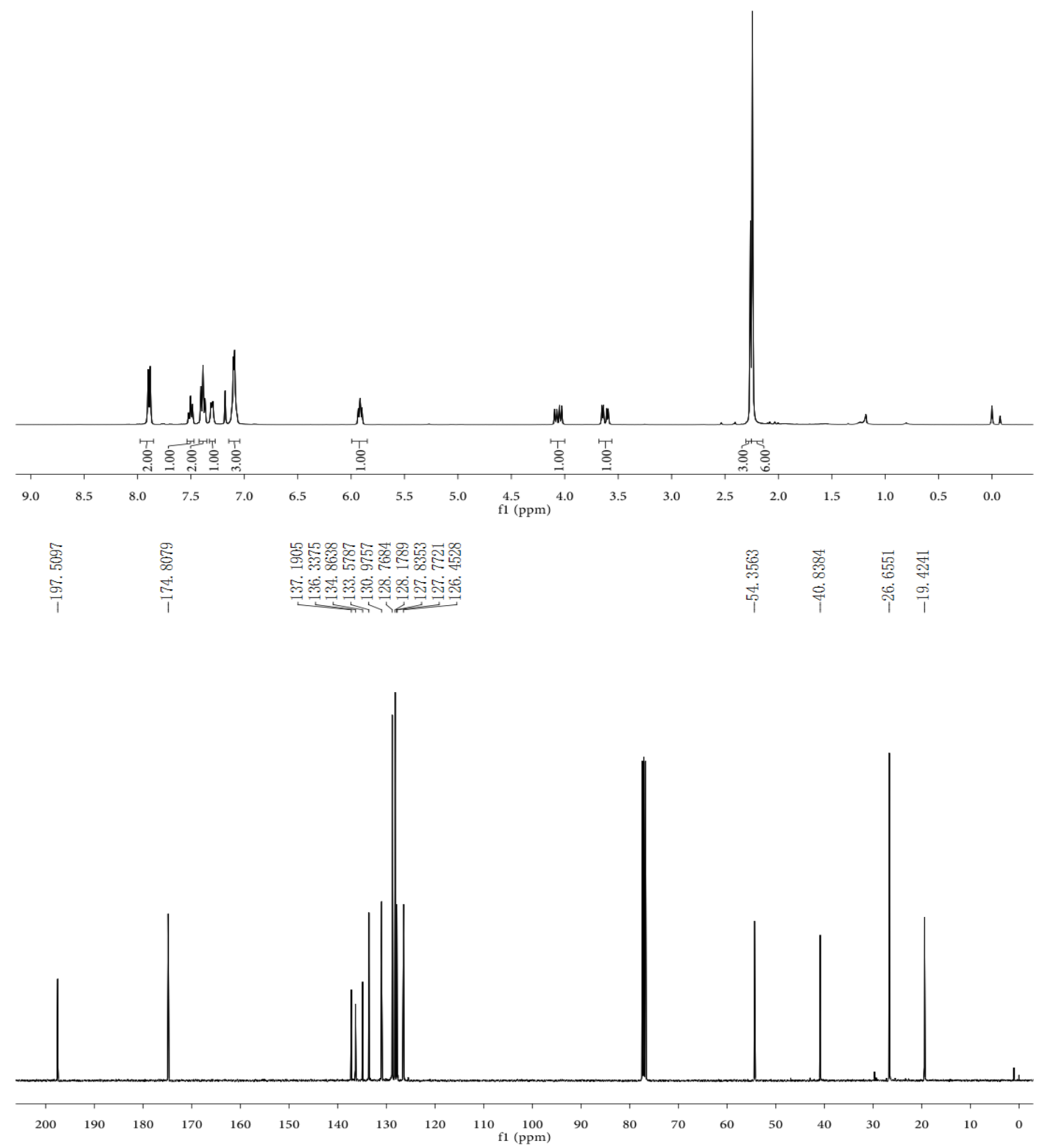


$N$-acetyl- $N$-(3-oxo-3-phenyl-1-(m-tolyl)propyl)acetamide (28):<smiles>CC(=O)N(C(C)=O)C(CC(=O)c1ccccc1)c1cccc(C)c1</smiles>

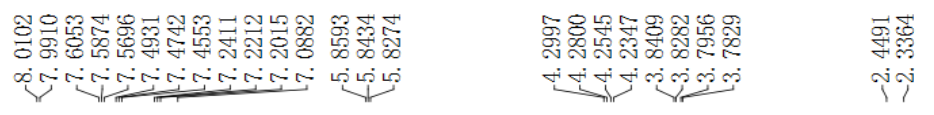
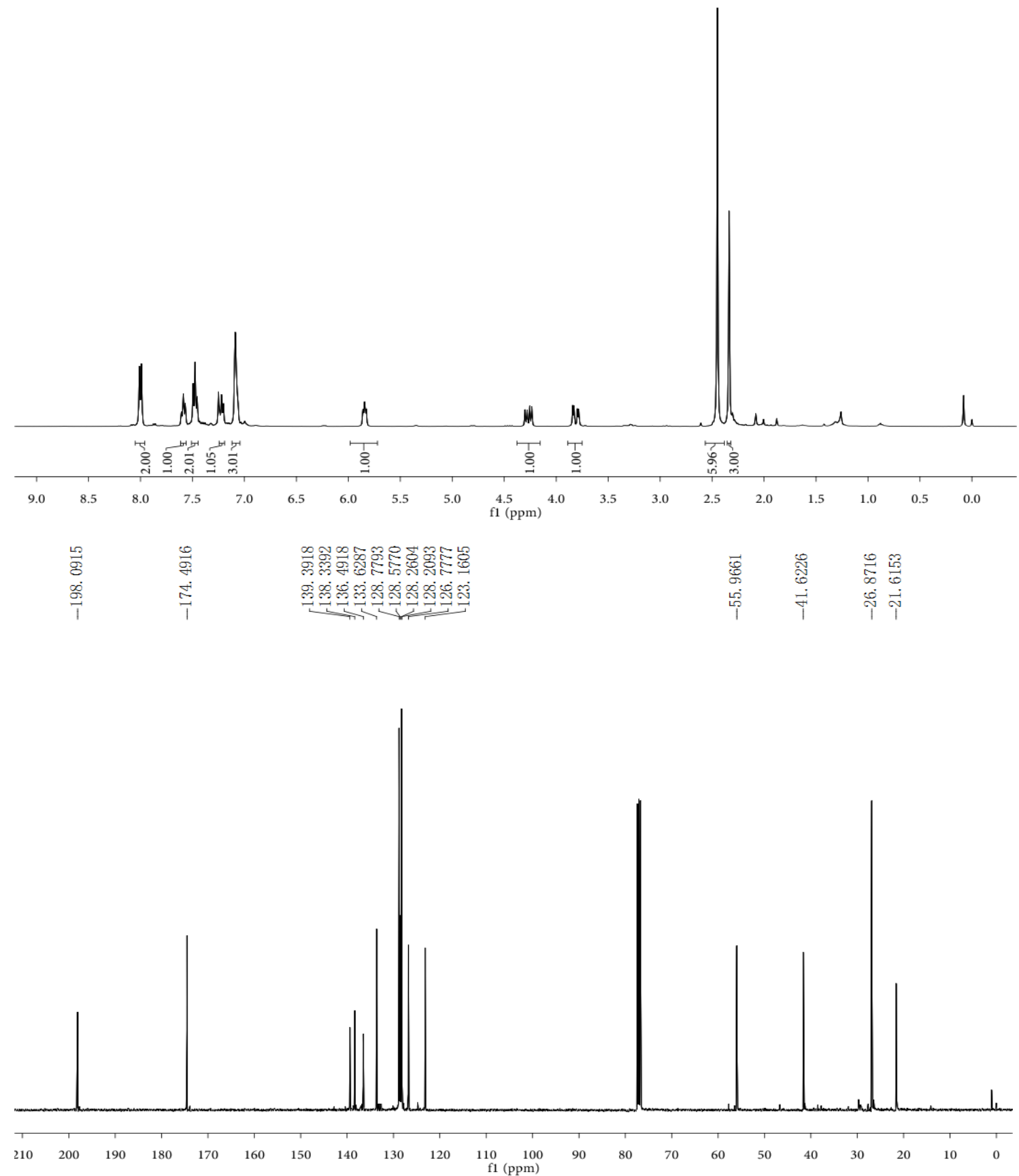
$N$-acetyl- $N$-(3-oxo-3-phenyl-1-(p-tolyl)propyl)acetamide (29):<smiles>CC(=O)N(C(C)=O)C(CC(=O)c1ccccc1)c1ccc(C)cc1</smiles>

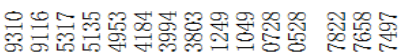

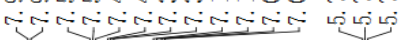

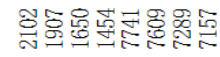

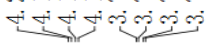

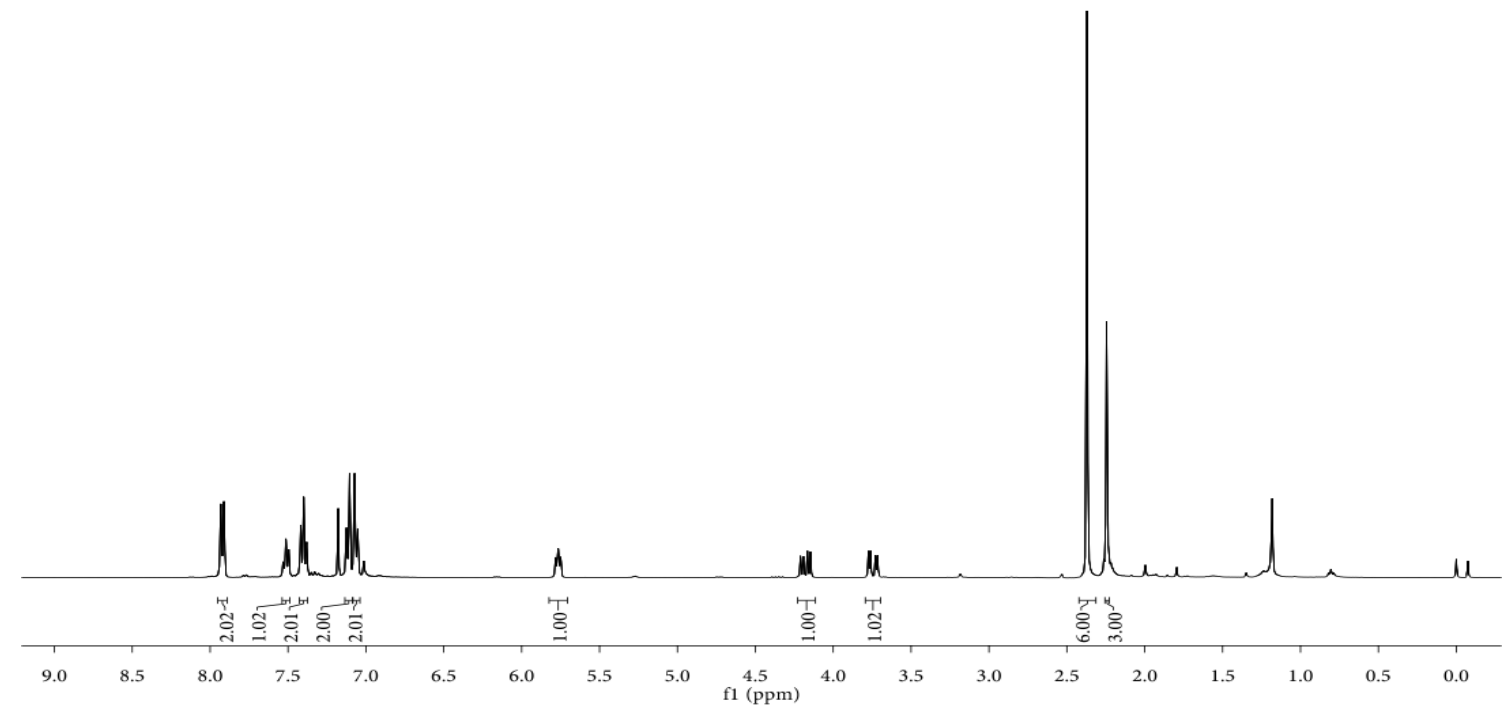

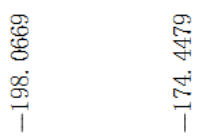

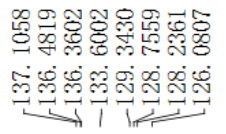

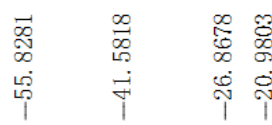

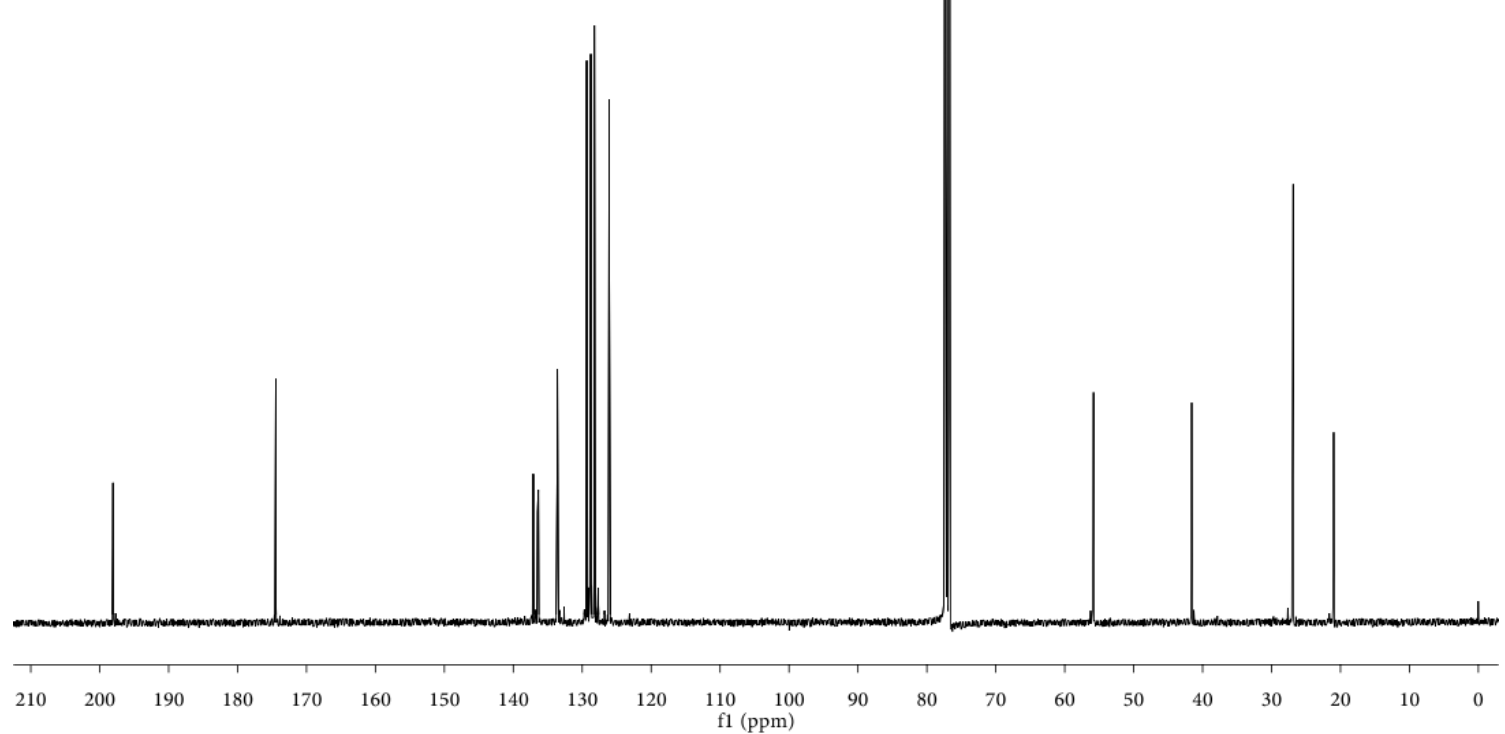


$N$-acetyl- $N$-(1-(2,5-dimethylphenyl)-3-oxo-3-phenylpropyl)acetamide (30):

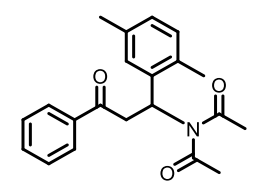

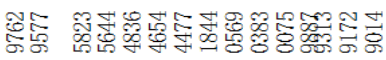

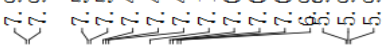

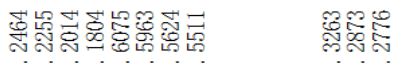

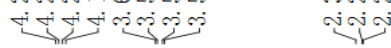
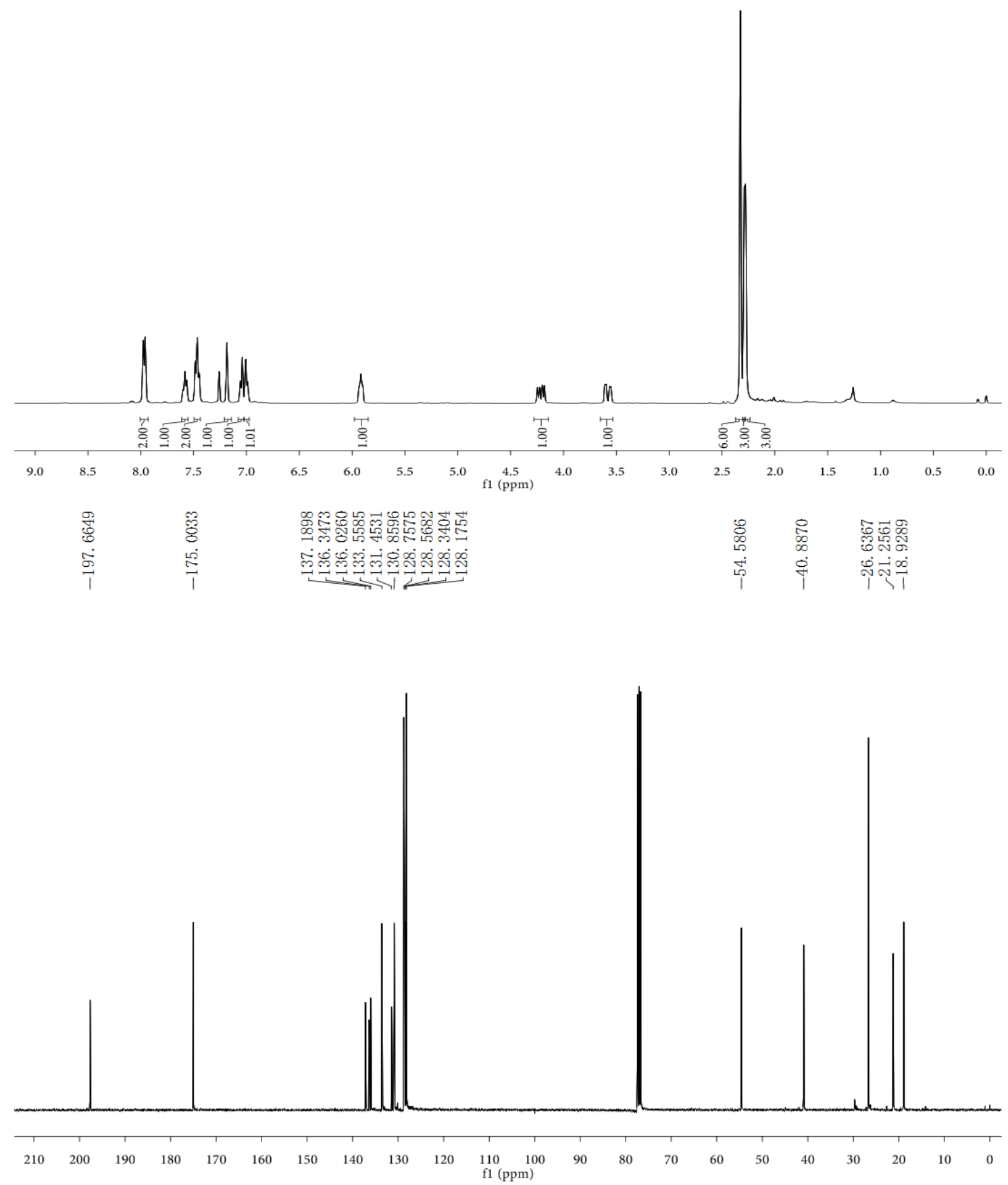
$N$-acetyl- $N$-(1-(4-(tert-butyl)phenyl)-3-oxo-3-phenylpropyl)acetamide (31):<smiles>CC(=O)N(C(C)=O)C(C(=O)c1ccccc1)c1ccc(C(C)(C)C)cc1</smiles>

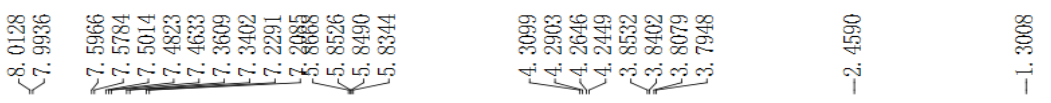

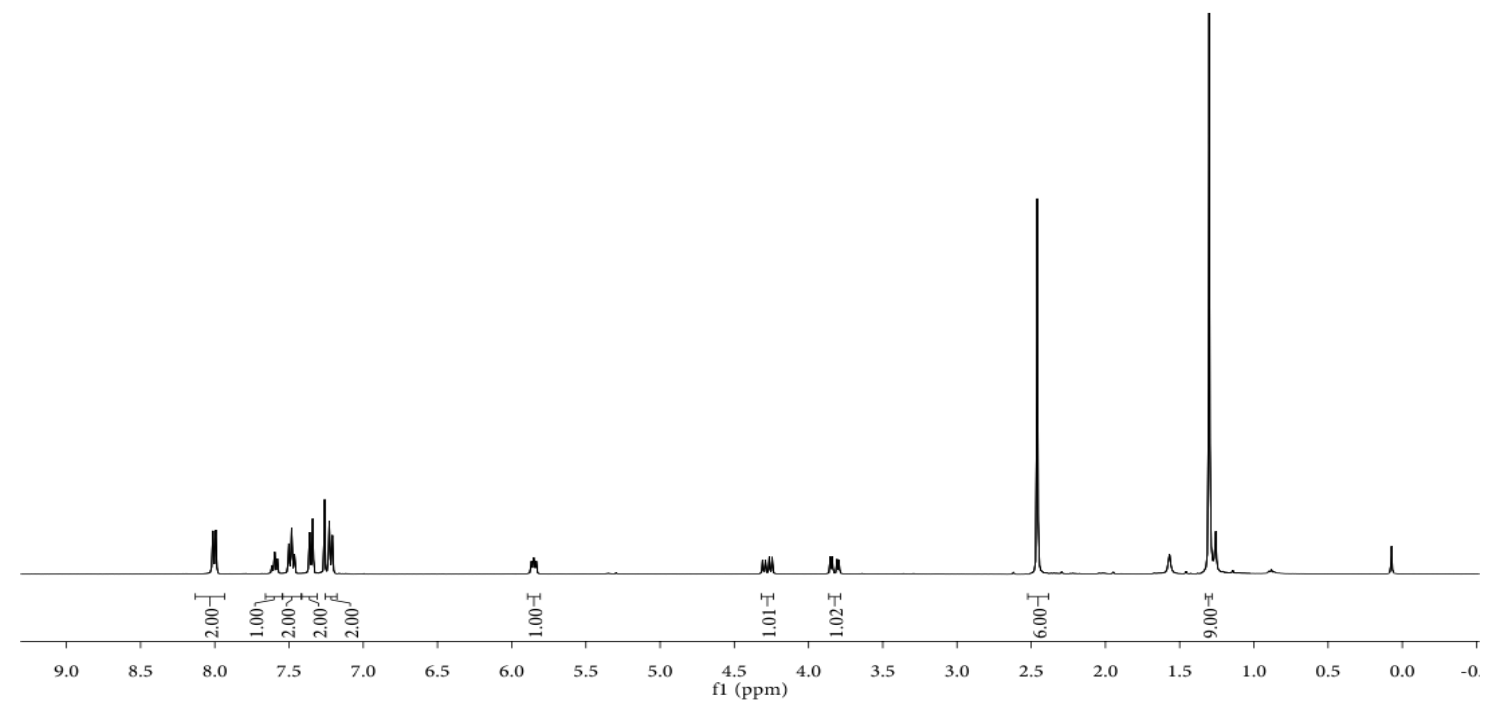

\begin{tabular}{|c|c|c|}
\hline 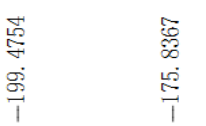 & 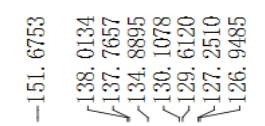 & 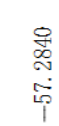 \\
\hline
\end{tabular}

$\begin{array}{llllllllllllllllllllll}210 & 200 & 190 & 180 & 170 & 160 & 150 & 140 & 130 & 120 & \begin{array}{l}110 \\ \mathrm{f} 1(\mathrm{ppm})\end{array} & 90 & 80 & 70 & 60 & 50 & 40 & 30 & 20 & 10 & 0\end{array}$ 
$N$-acetyl- $N$-(1-(2-fluorophenyl)-3-oxo-3-phenylpropyl)acetamide (32):
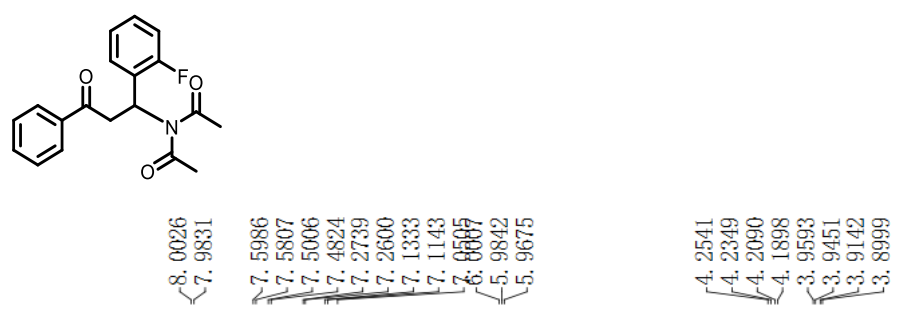

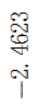

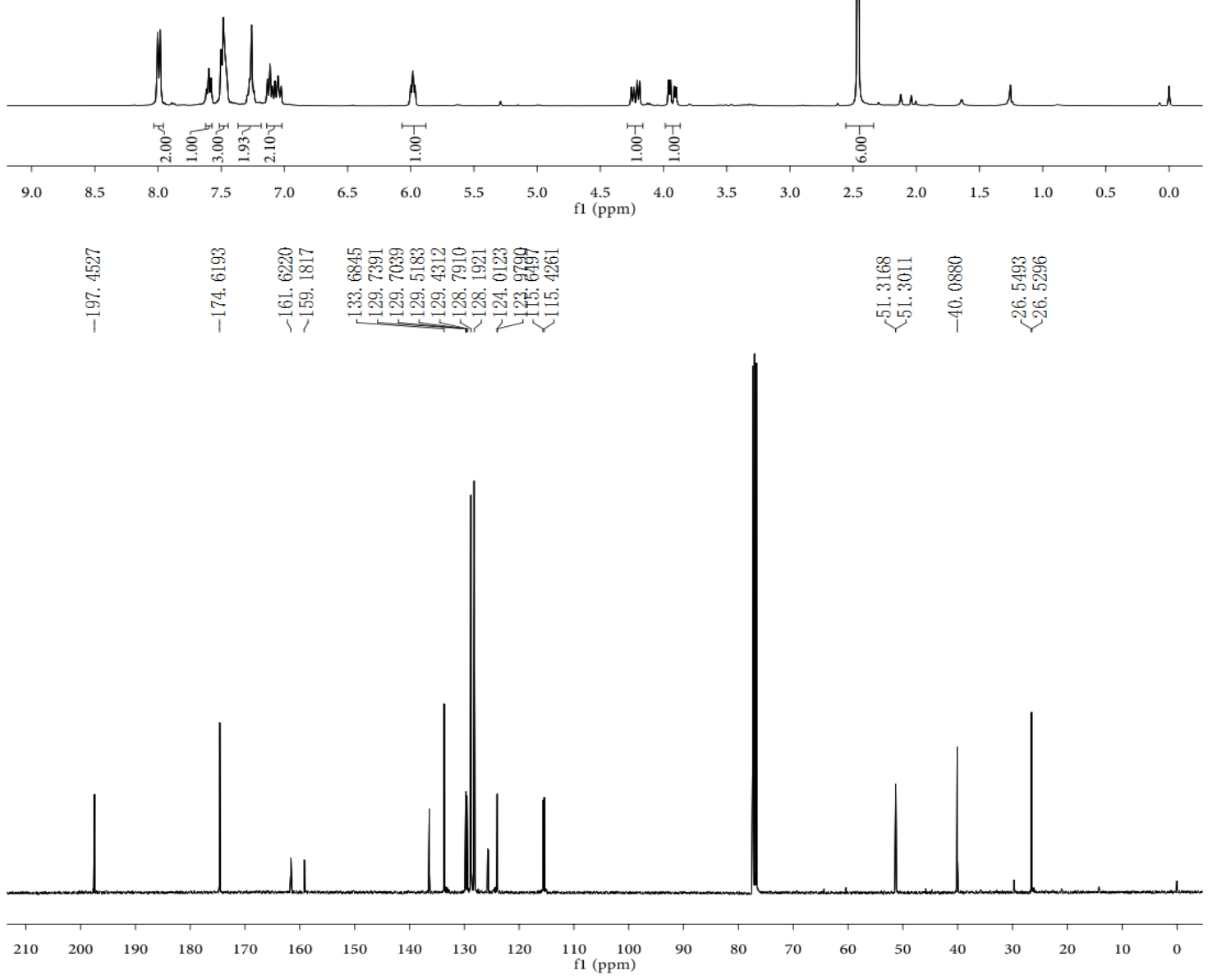

S62 


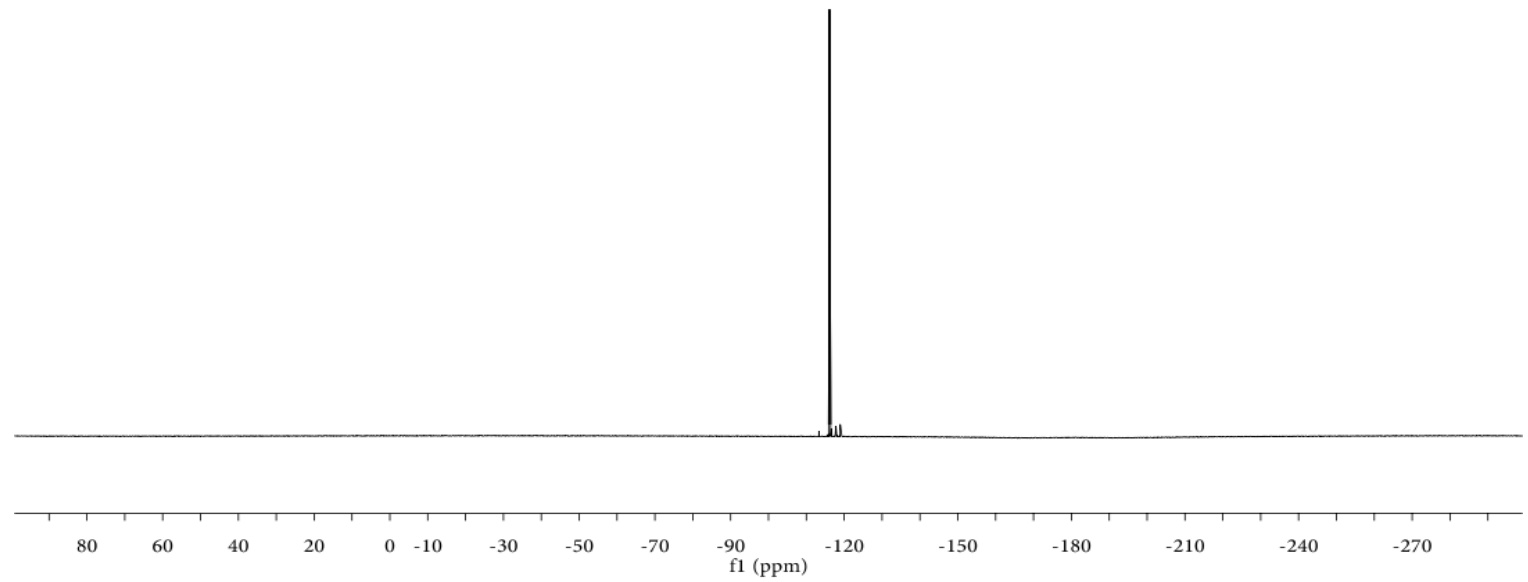


$N$-acetyl- $N$-(1-(4-fluorophenyl)-3-oxo-3-phenylpropyl)acetamide (33):
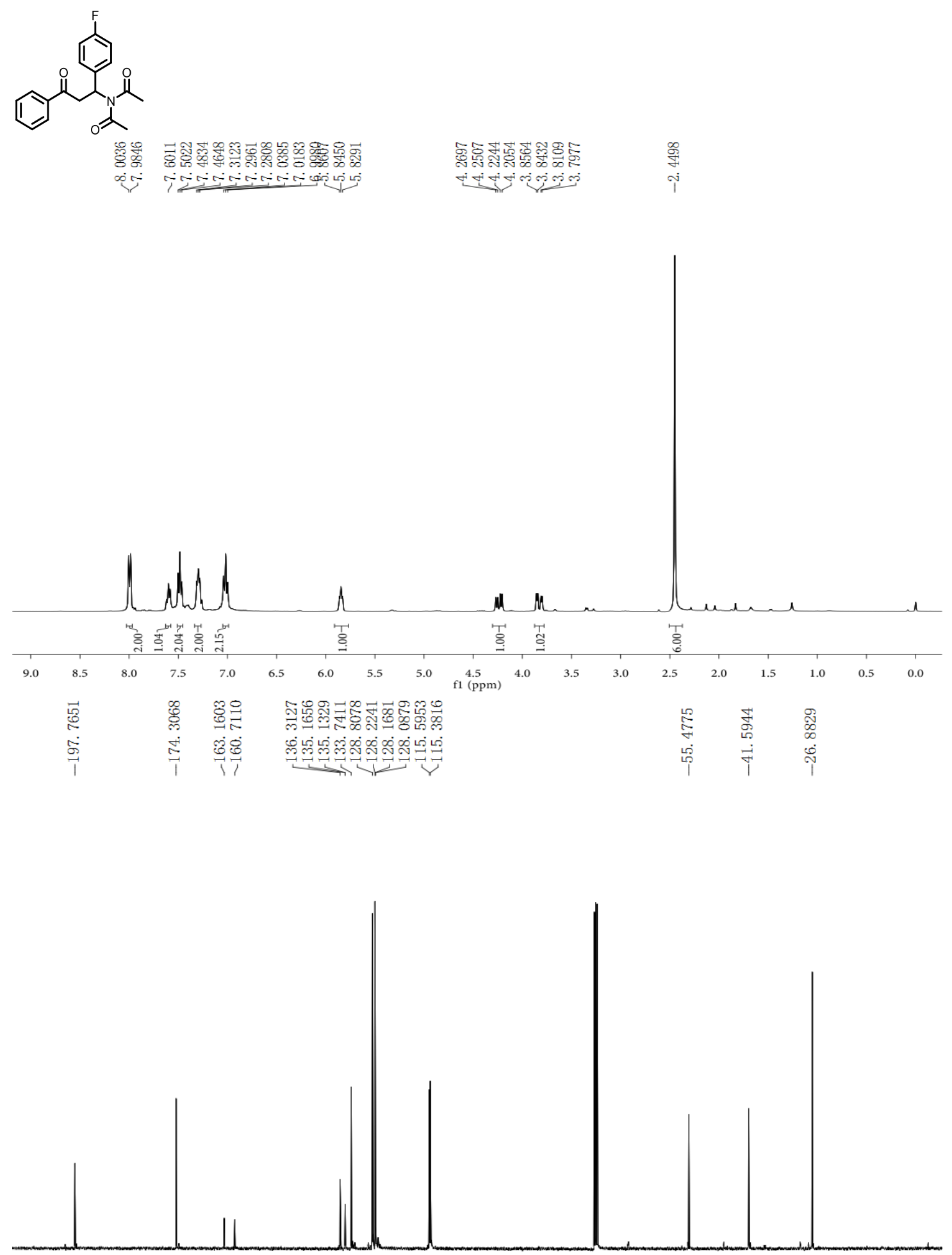

2

$\begin{array}{lllllllllll}200 & 190 & 180 & 170 & 160 & 150 & 140 & 130 & 120 & \begin{array}{c}110 \\ \mathrm{f1}(\mathrm{ppm})\end{array}\end{array}$ 


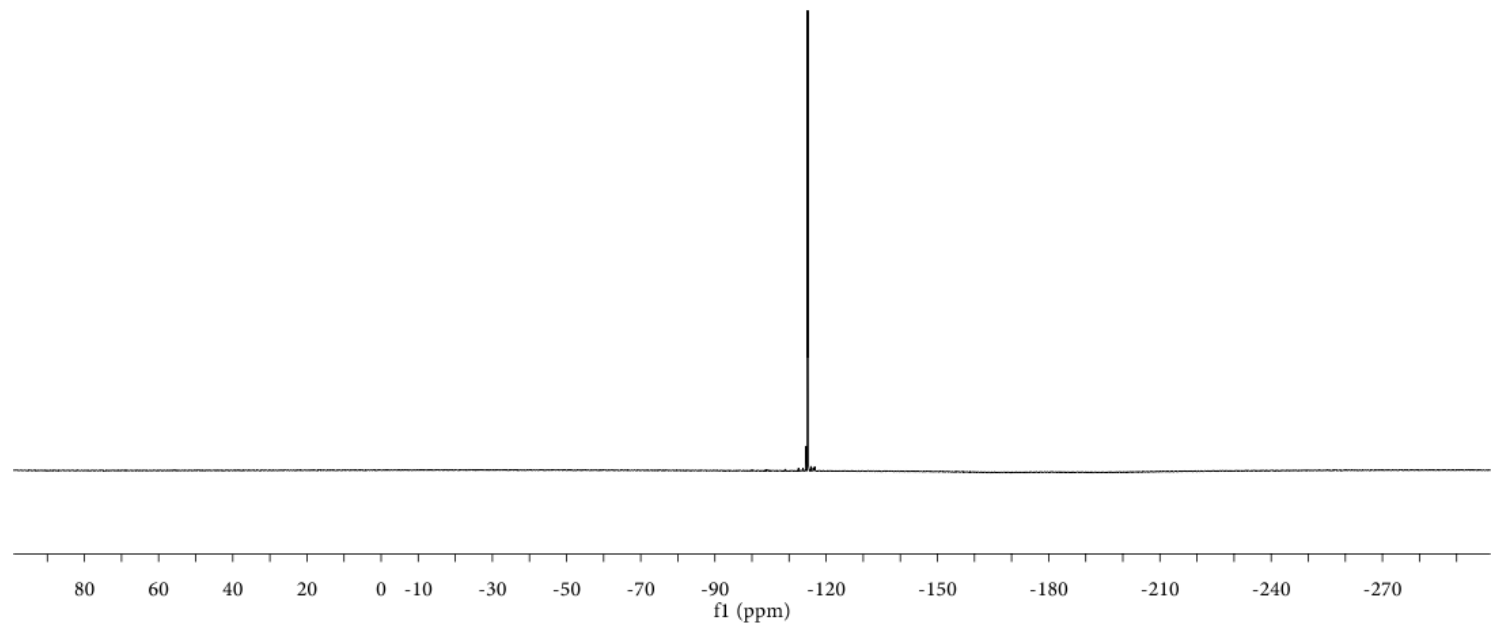


$N$-acetyl- $N$-(1-(4-chlorophenyl)-3-oxo-3-phenylpropyl)acetamide (34):<smiles>CC(=O)N(C(C)=O)C(CC(=O)c1ccccc1)c1ccc(Cl)cc1</smiles>

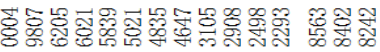

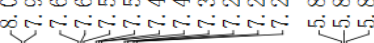

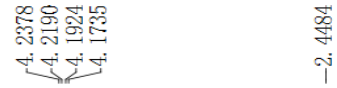

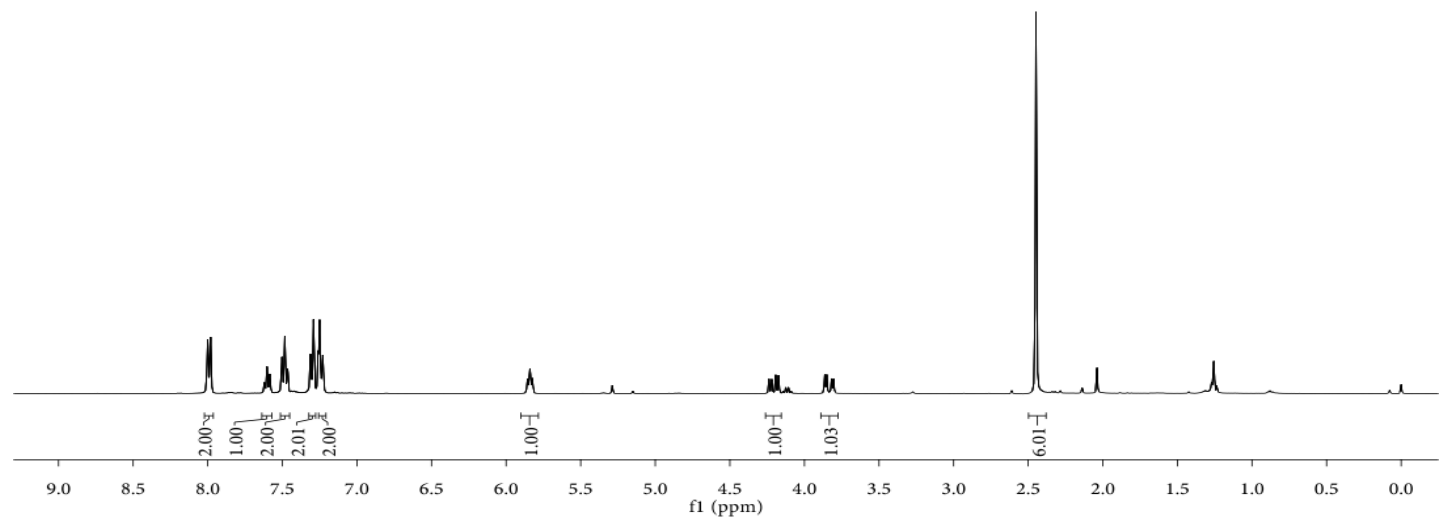

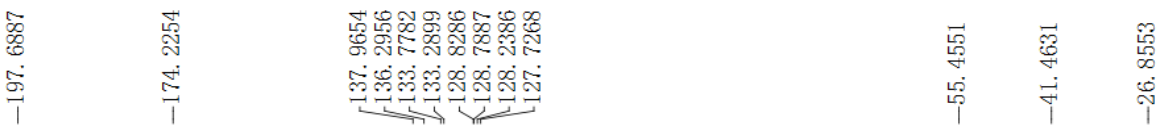

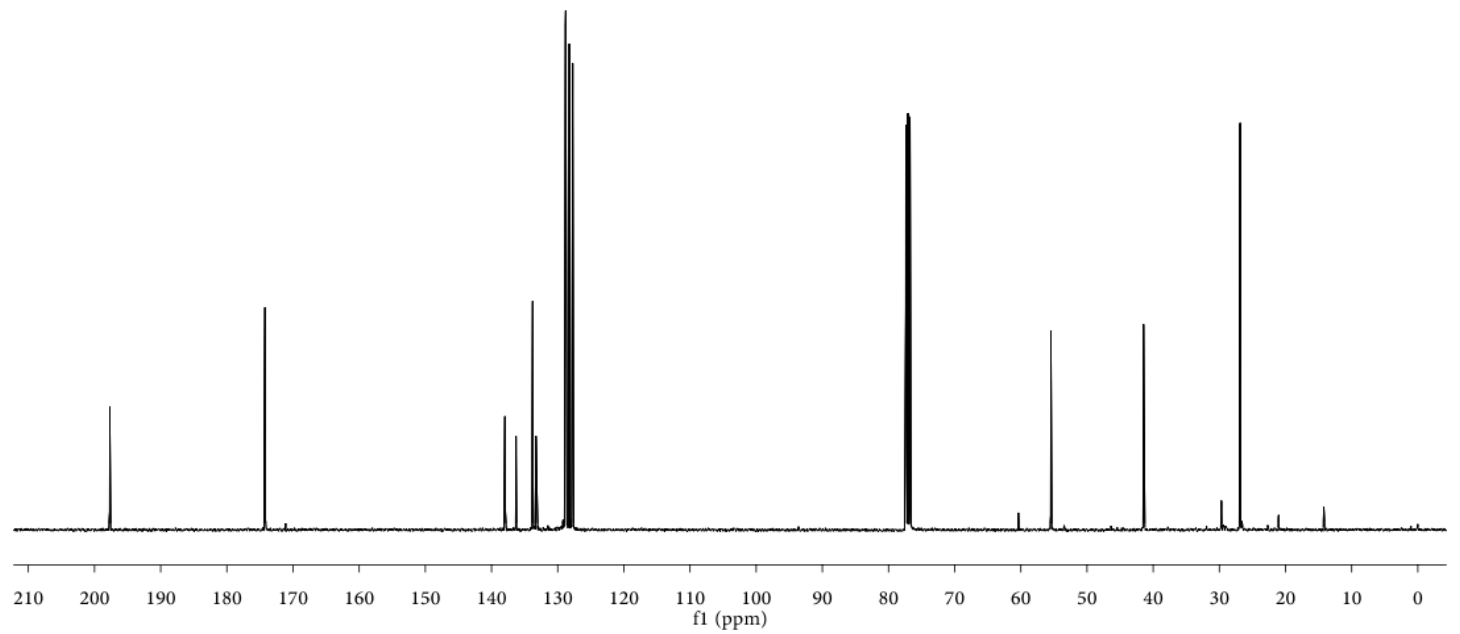


$N$-acetyl- $N$-(1-(4-bromophenyl)-3-oxo-3-phenylpropyl)acetamide (35):<smiles>CC(=O)N(C(C)=O)C(CC(=O)c1ccccc1)c1ccc(Br)cc1</smiles>

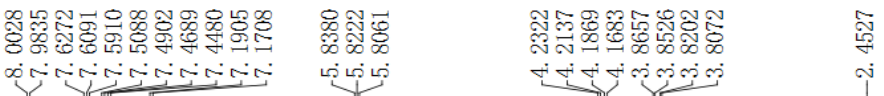

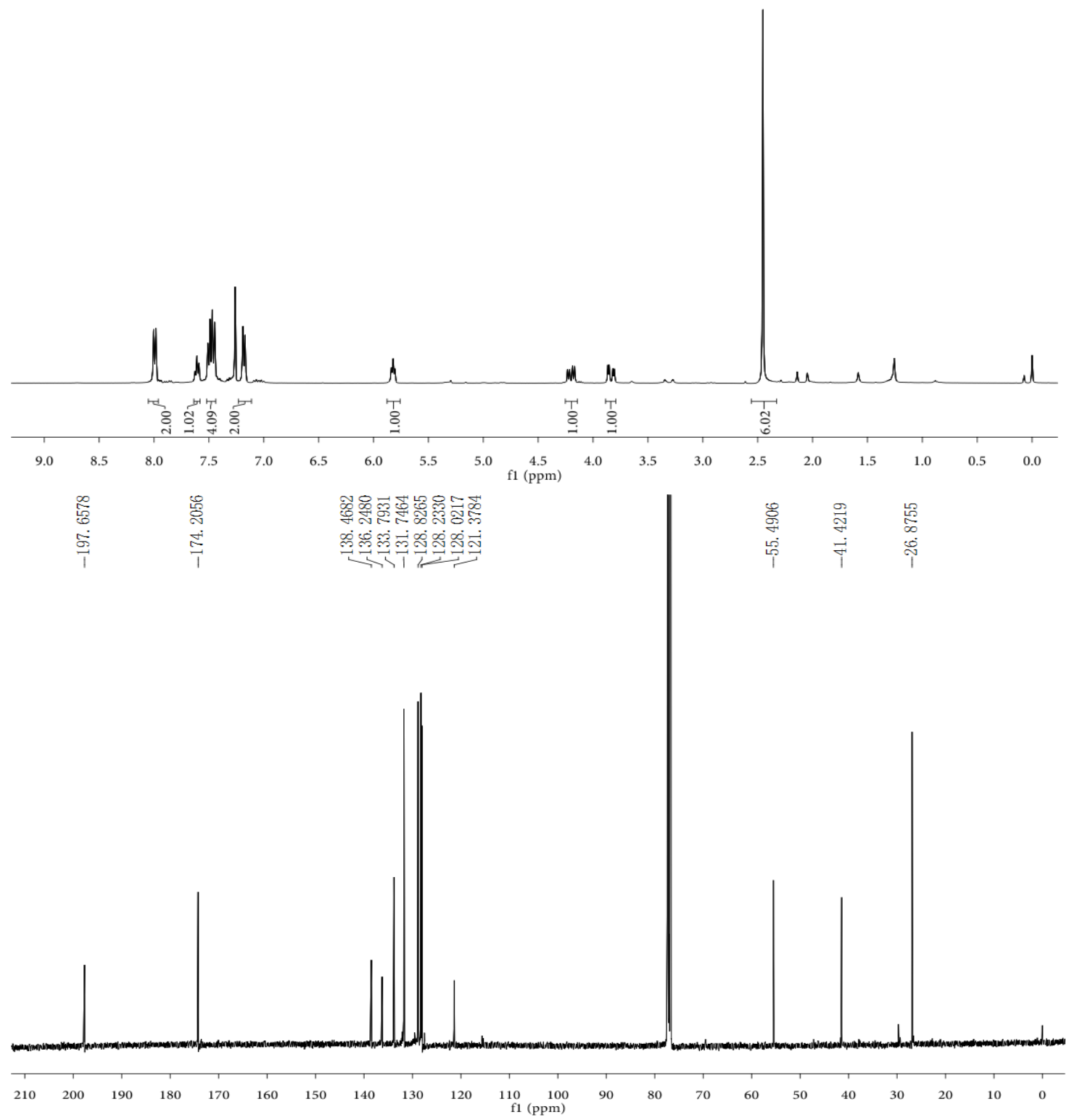


$N$-(1-([1,1'-biphenyl]-4-yl)-3-oxo-3-phenylpropyl)- $N$-acetylacetamide (36):

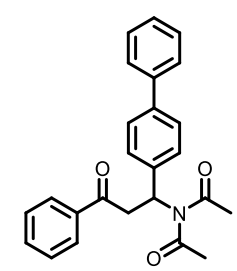

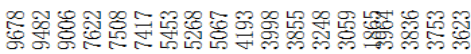

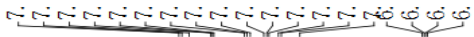

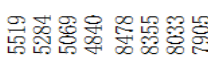

भififim
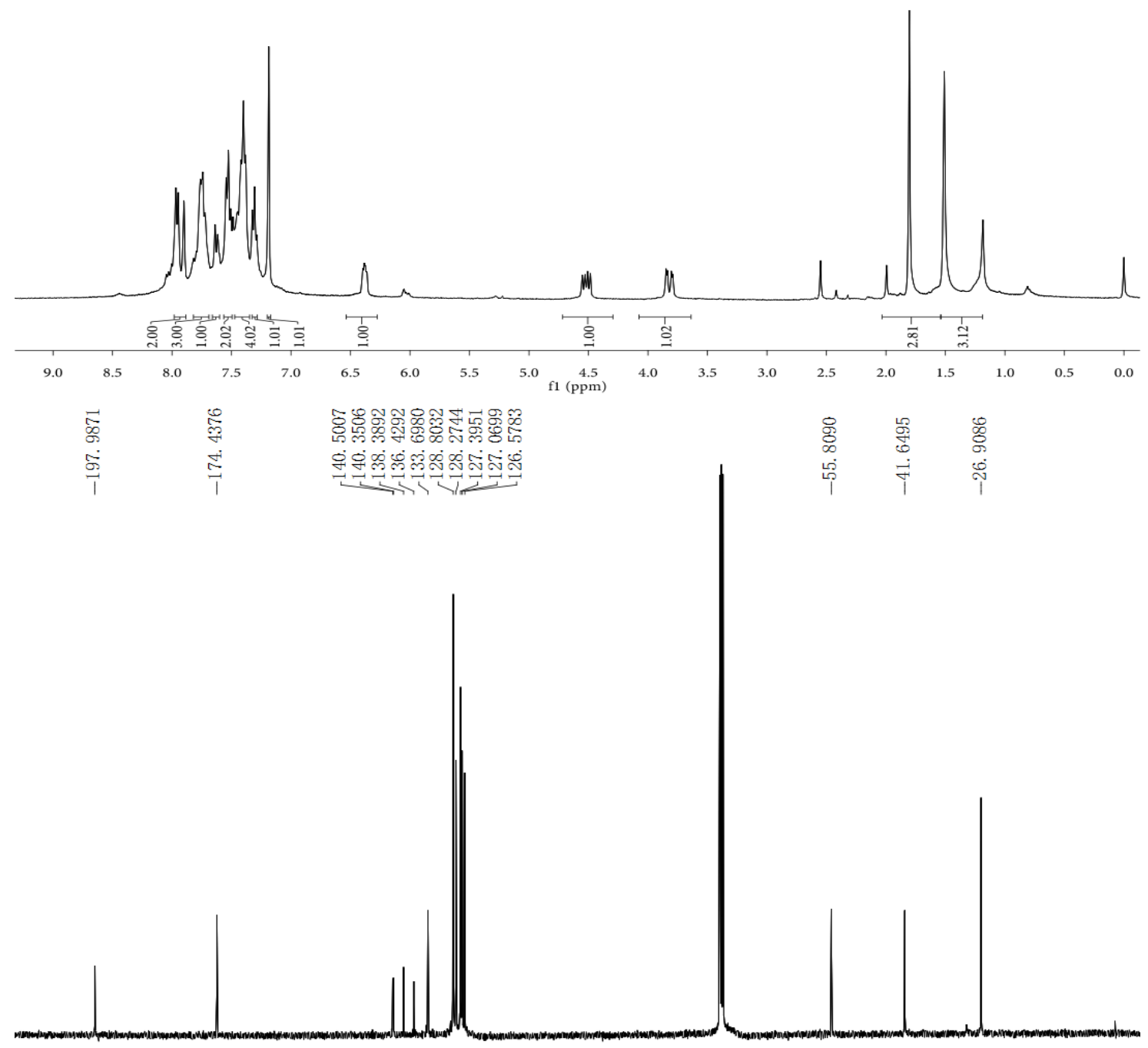

$\begin{array}{lllllllllllllllllllll}210 & 200 & 190 & 180 & 170 & 160 & 150 & 140 & 130 & 120 & \begin{array}{c}110 \\ \mathrm{f} 1(\mathrm{ppm})\end{array} & 90 & 80 & 70 & 60 & 50 & 40 & 30 & 20 & 10 & 0\end{array}$ 
$N$-acetyl- $N$-(1-(naphthalen-2-yl)-3-oxo-3-phenylpropyl)acetamide(37):<smiles>CC(=O)N(CC(=O)c1ccccc1)C(C)(C)C(=O)c1ccc2ccccc2c1</smiles>

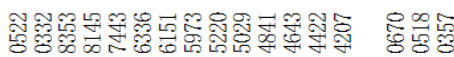

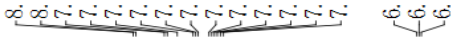

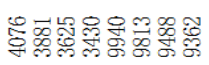

मifitim

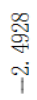

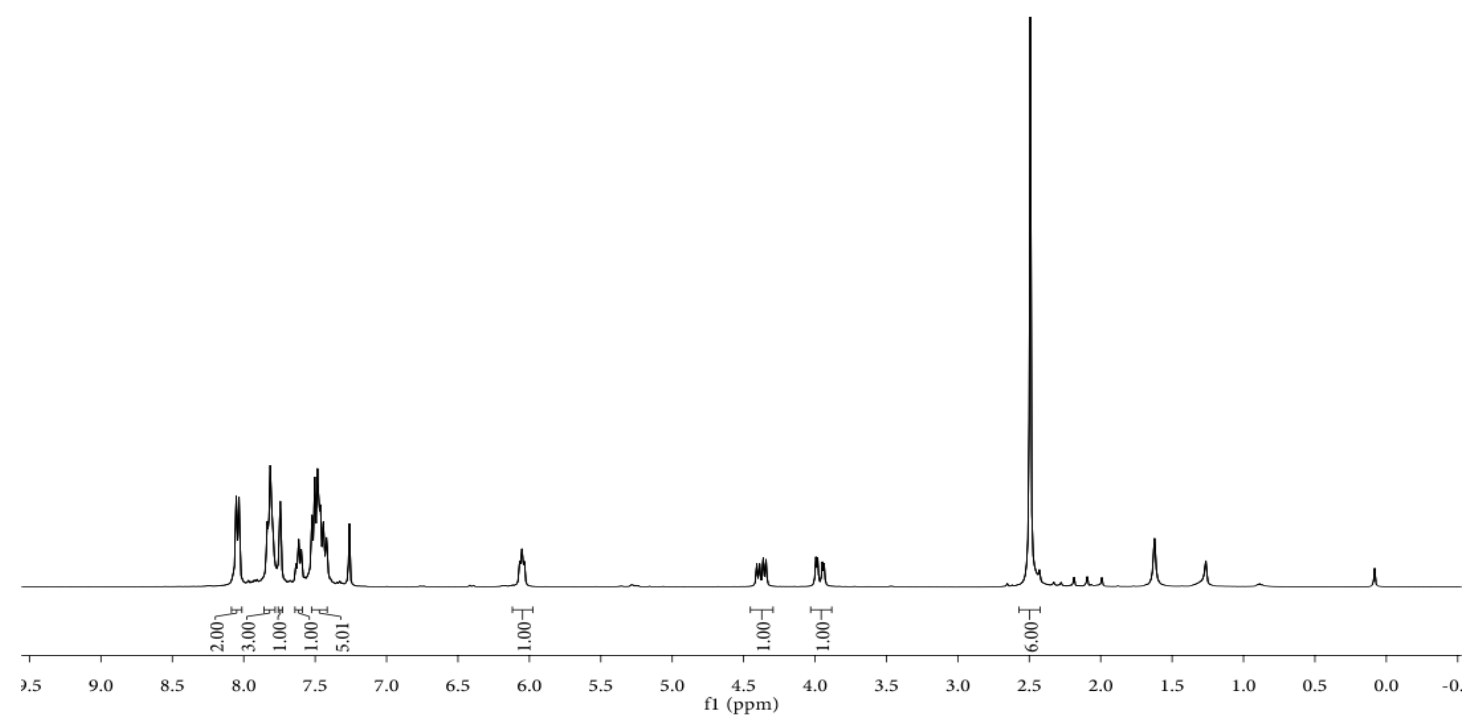

$\begin{array}{lllllllllll}210 & 200 & 190 & 180 & 170 & 160 & 150 & 140 & 130 & 120 & \begin{array}{l}110 \\ \mathrm{f} 1(\mathrm{ppm})\end{array}\end{array}$

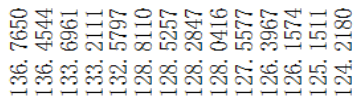

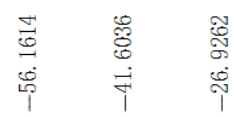

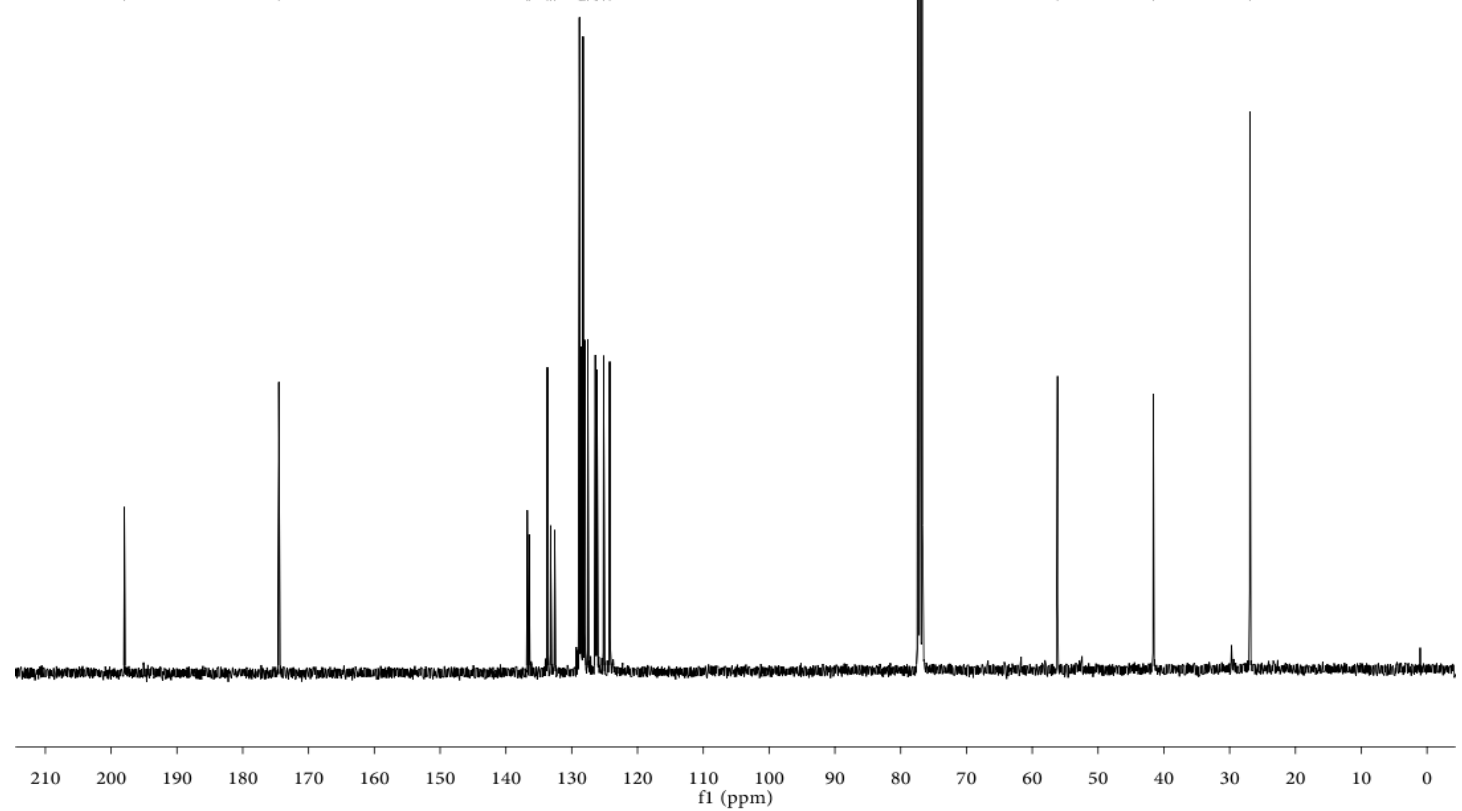


$N$-acetyl- $N$-(3-oxo-1,3-diphenylpropyl)butyramide (38):

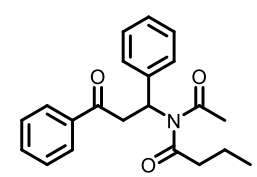

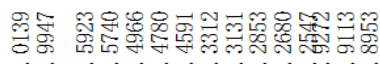

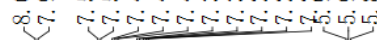

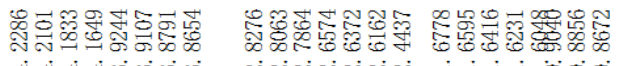

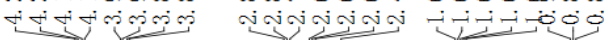
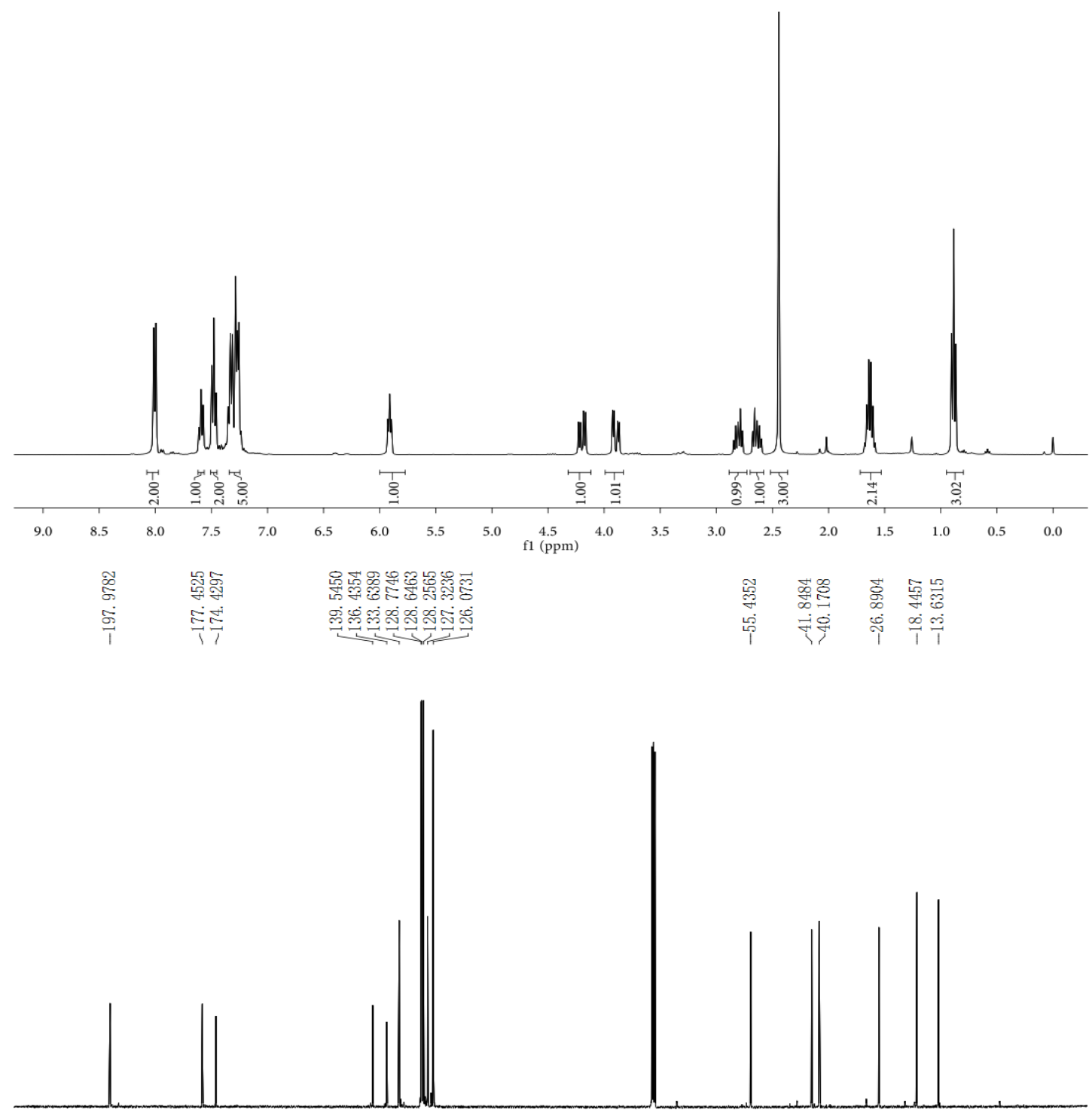

$\begin{array}{llllllllllllllllllllllll}210 & 200 & 190 & 180 & 170 & 160 & 150 & 140 & 130 & 120 & 110 & \begin{array}{c}100 \\ \mathrm{f} 1(\mathrm{ppm})\end{array} & 90 & 80 & 70 & 60 & 50 & 40 & 30 & 20 & 10 & 0 & -10\end{array}$ 
$N$-acetyl- $N$-(3-oxo-1,3-diphenylpropyl)pentanamide (39):

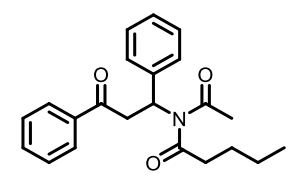

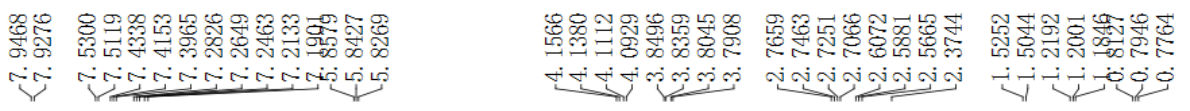
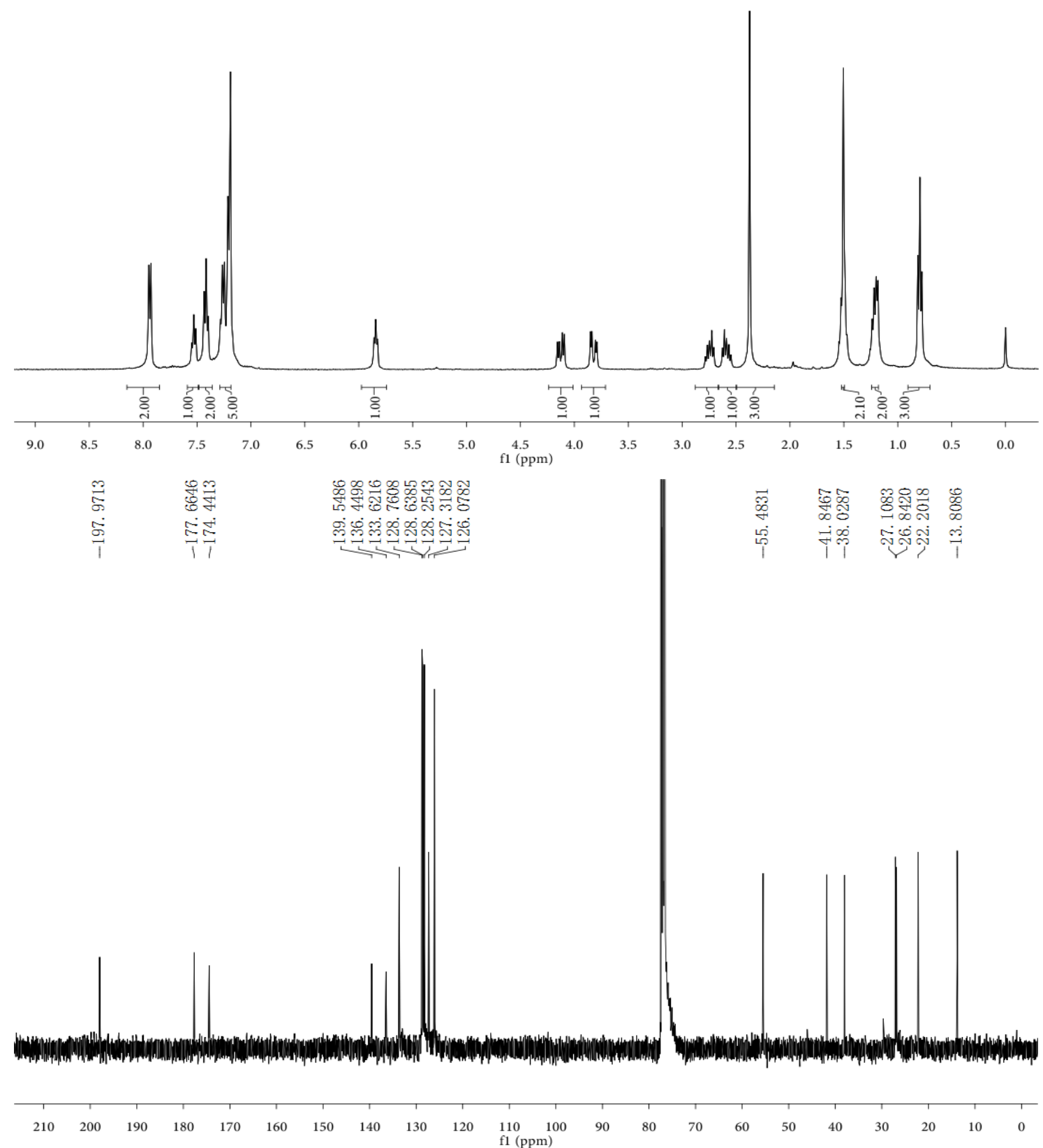
$N$-acetyl- $N$-(3-oxo-1,3-diphenylpropyl)isobutyramide (40):<smiles>CC(=O)N(C(=O)CC(C(=O)c1ccccc1)c1ccccc1)C(C)C(C)C</smiles>

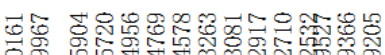

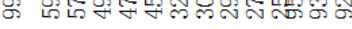

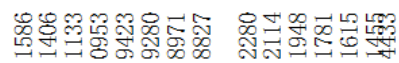

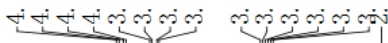

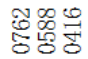

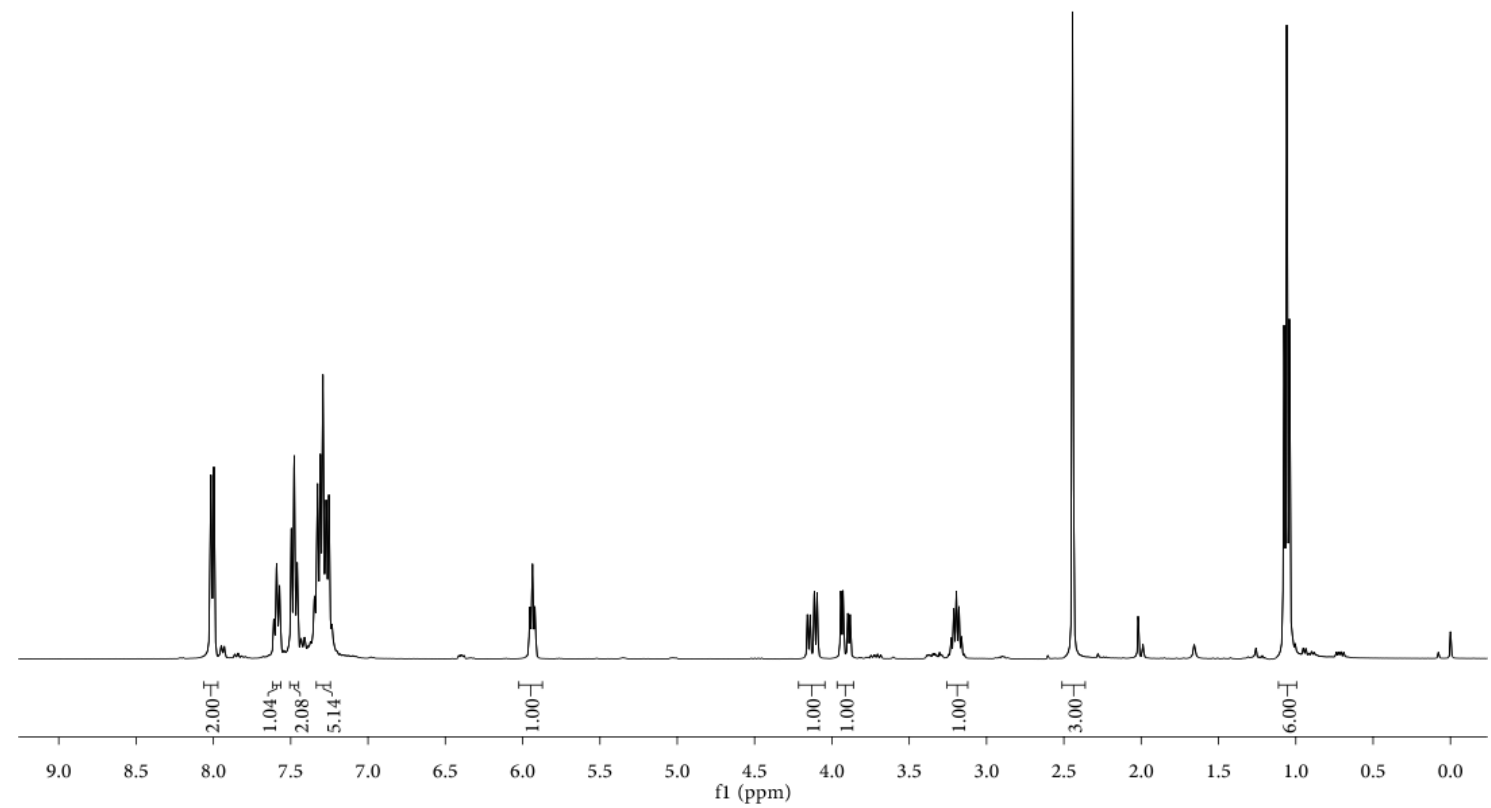

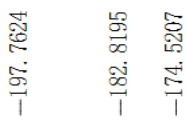

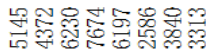

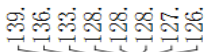

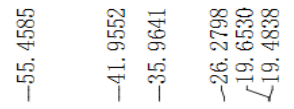

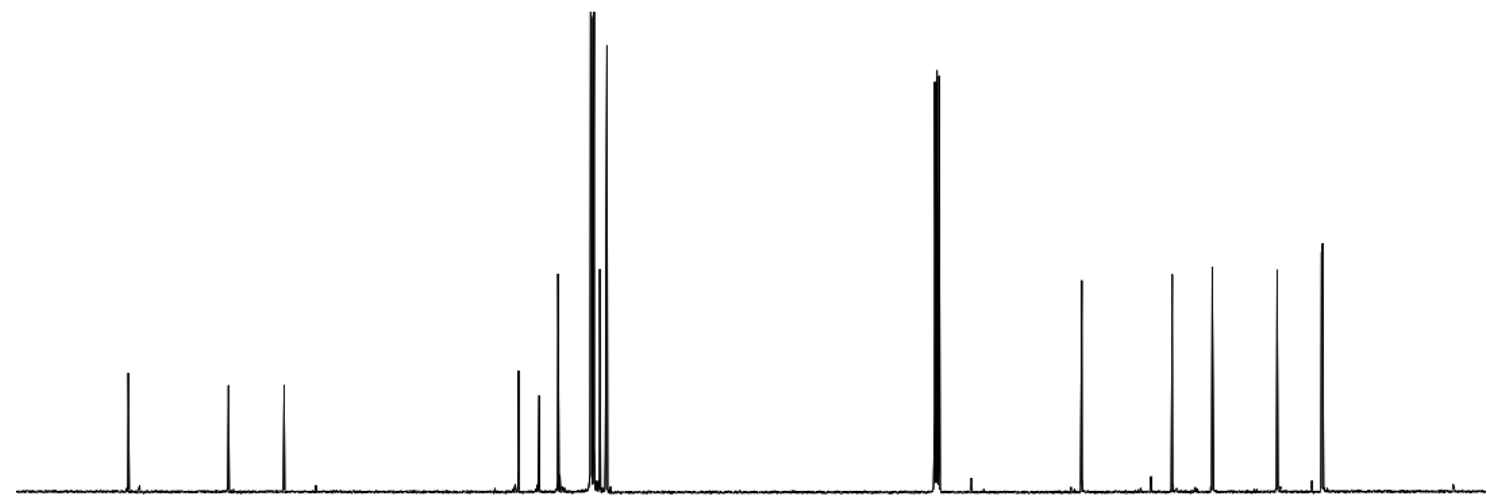
$\begin{array}{lllllllllll}210 & 200 & 190 & 180 & 170 & 160 & 150 & 140 & 130 & 120 & \begin{array}{c}110 \\ \mathrm{f} 1(\mathrm{ppm})\end{array}\end{array}$ 
$N$-acetyl- $N$-(3-oxo-1,3-diphenylpropyl)cyclopropanecarboxamide (20'):<smiles>CC(=O)N(C(=O)C1CC1)C(CC(=O)c1ccccc1)c1ccccc1</smiles>

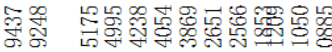

is initivitioge

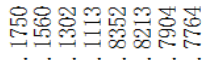

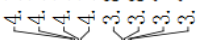

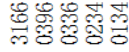

ช่ํ่ง
냉하용

iெi
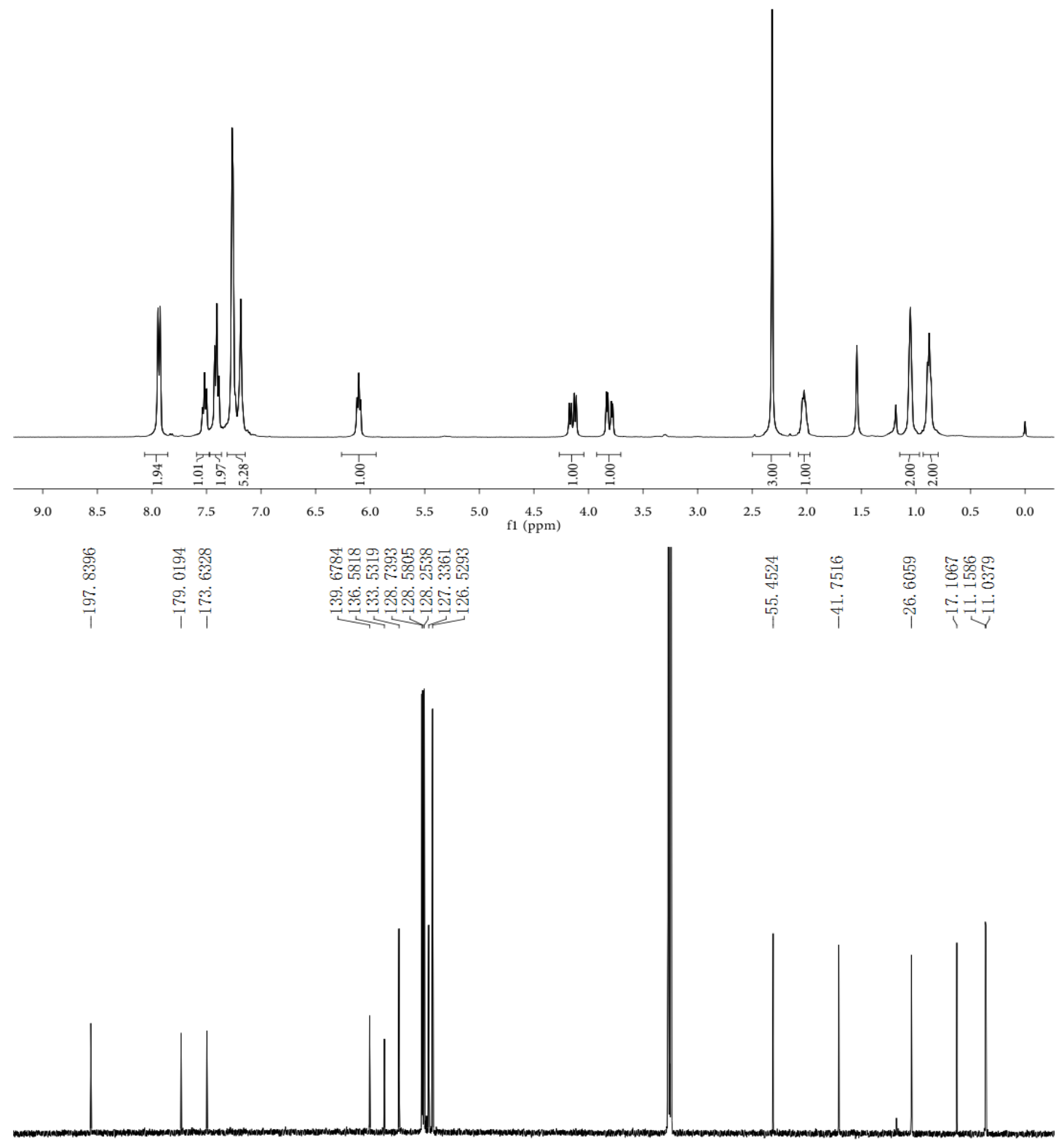

$\begin{array}{llllllllllll}210 & 200 & 190 & 180 & 170 & 160 & 150 & 140 & 130 & 120 & 110 \quad 10 \\ \text { f1 (ppm) }\end{array}$ 
$N$-acetyl- $N$-(3-oxo-1,3-diphenylpropyl)acetamide-d 3 (41):<smiles>CC(=O)N(C(C)=O)C(CC(=O)c1ccccc1)c1ccccc1</smiles>

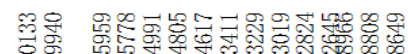

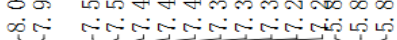

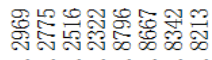

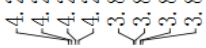

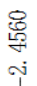

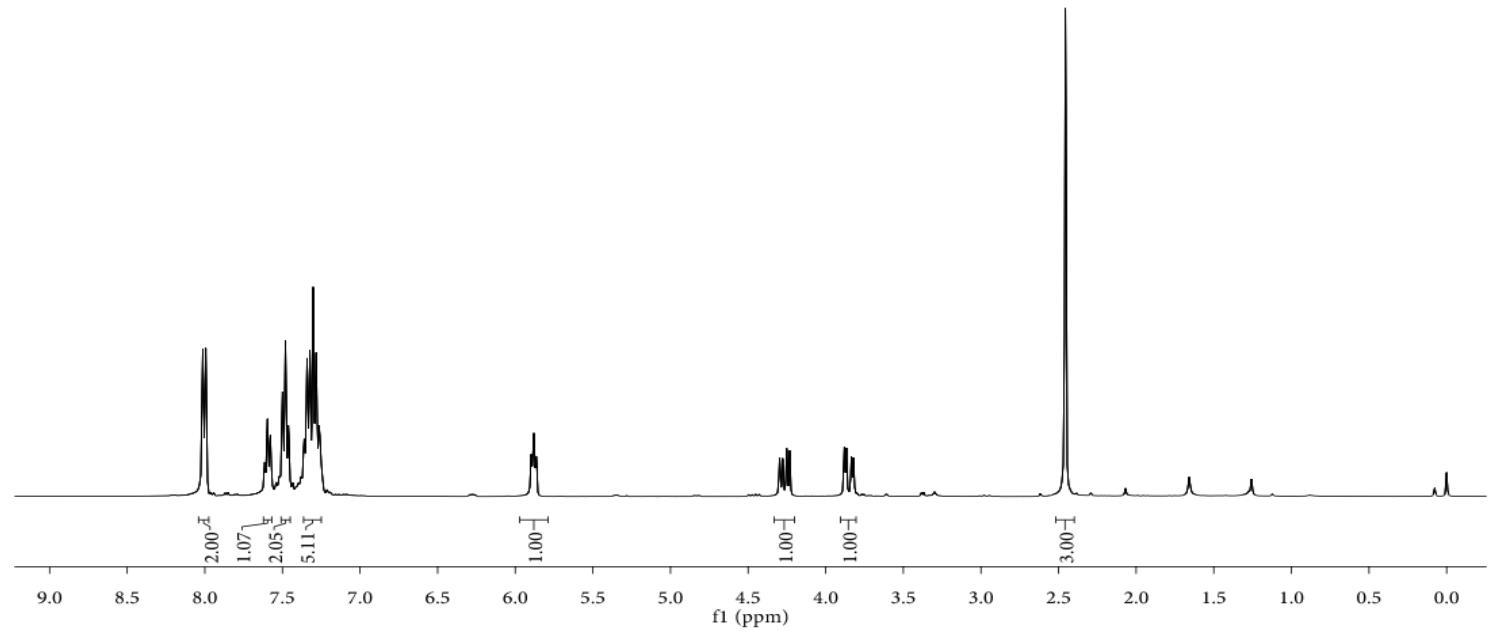

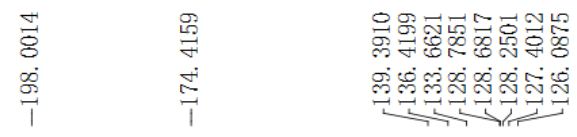

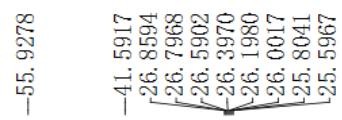

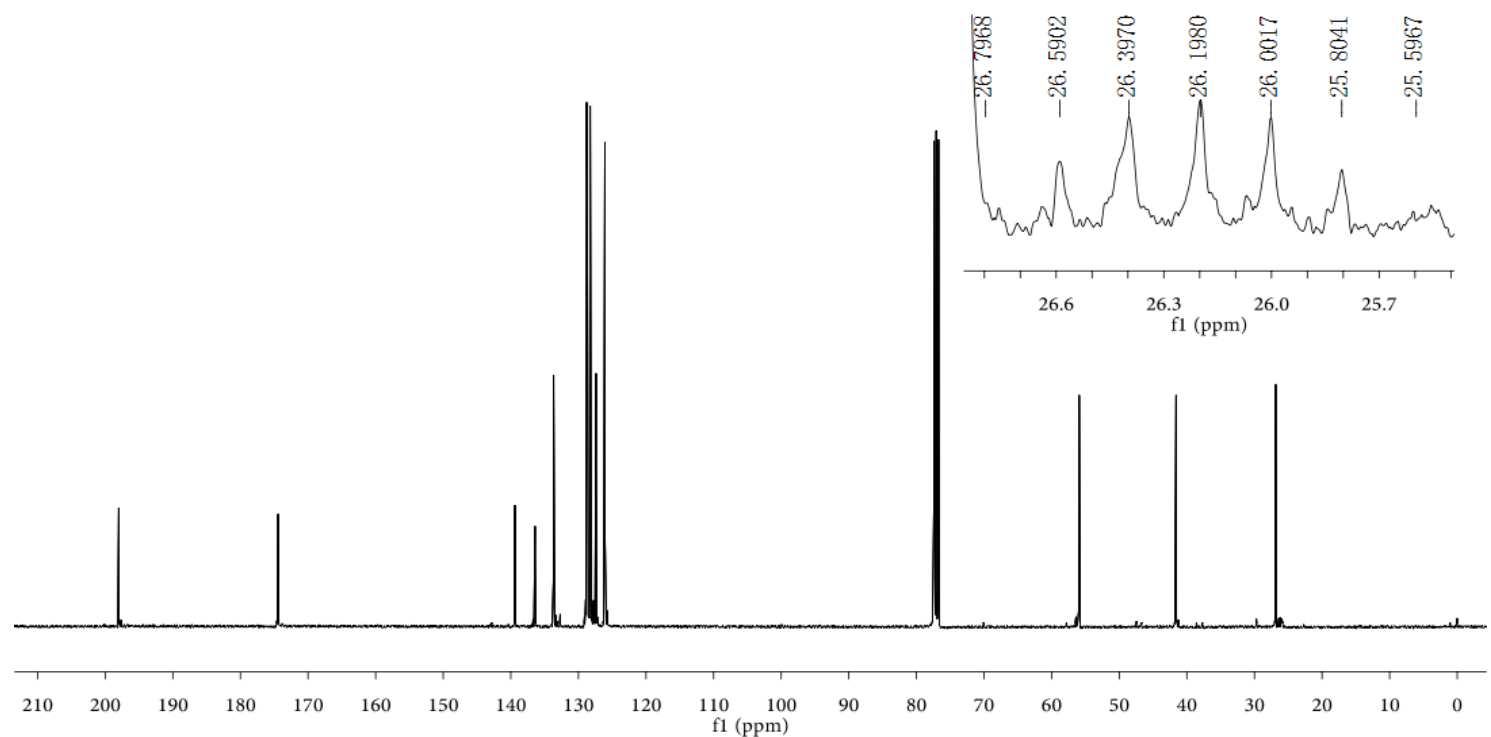


$N$-acetyl- $N$-(3-oxo-1,3-diphenylpropyl)acetamide-d3 (41'):<smiles></smiles>

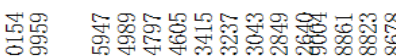

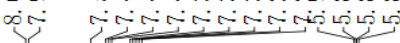

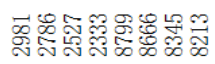

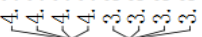

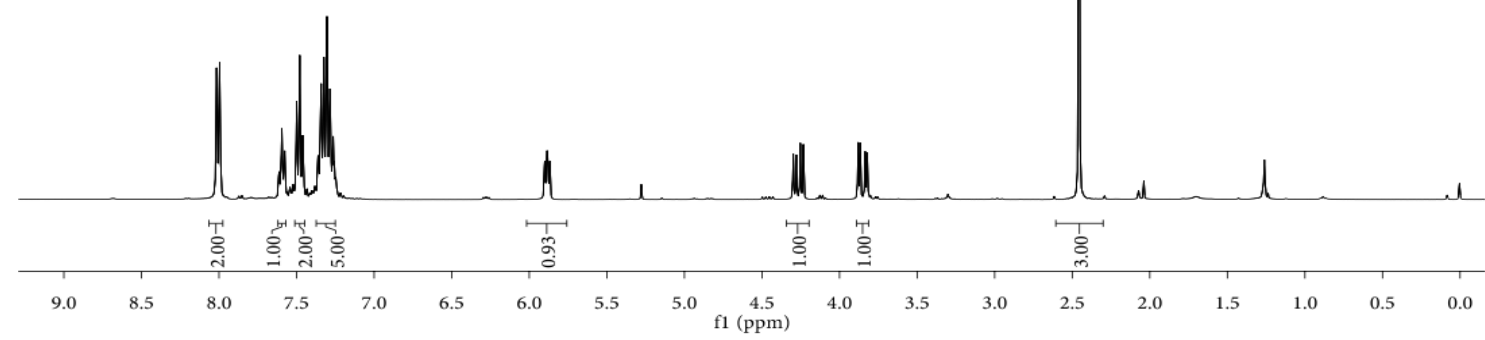

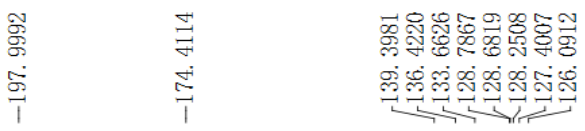

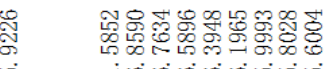

它

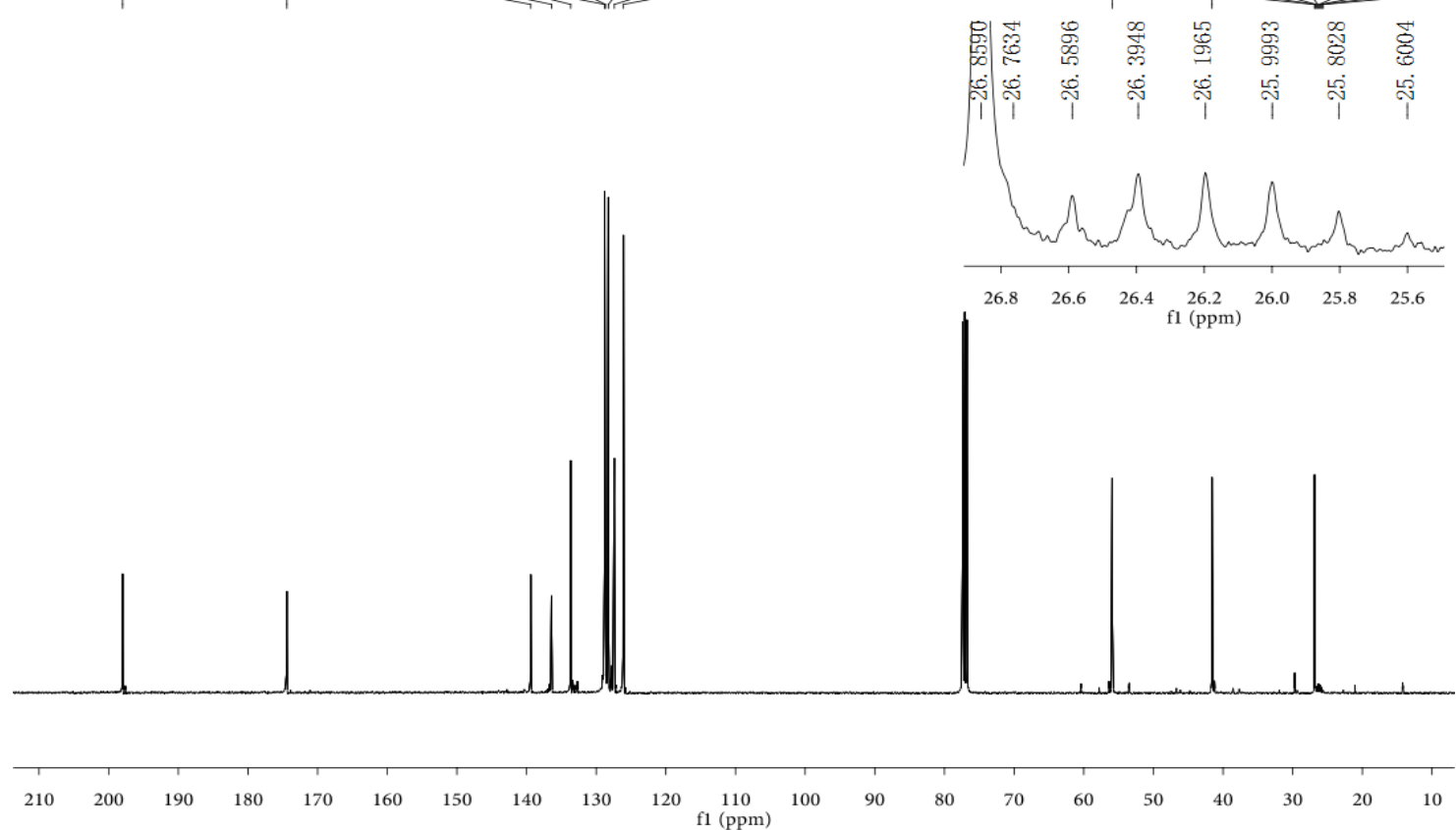


$N$-acetyl- $N$-(5-cyano-1-phenylpentyl)-4-(trifluoromethyl)benzamide (42):<smiles>CCCCCC(c1ccccc1)N(C(C)=O)C(=O)c1ccc(C(F)(F)F)cc1</smiles>

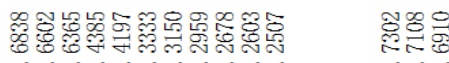

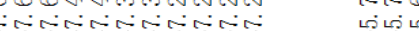

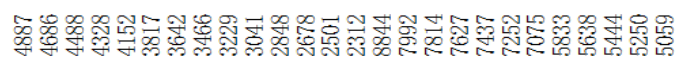
ง่ง ง ง
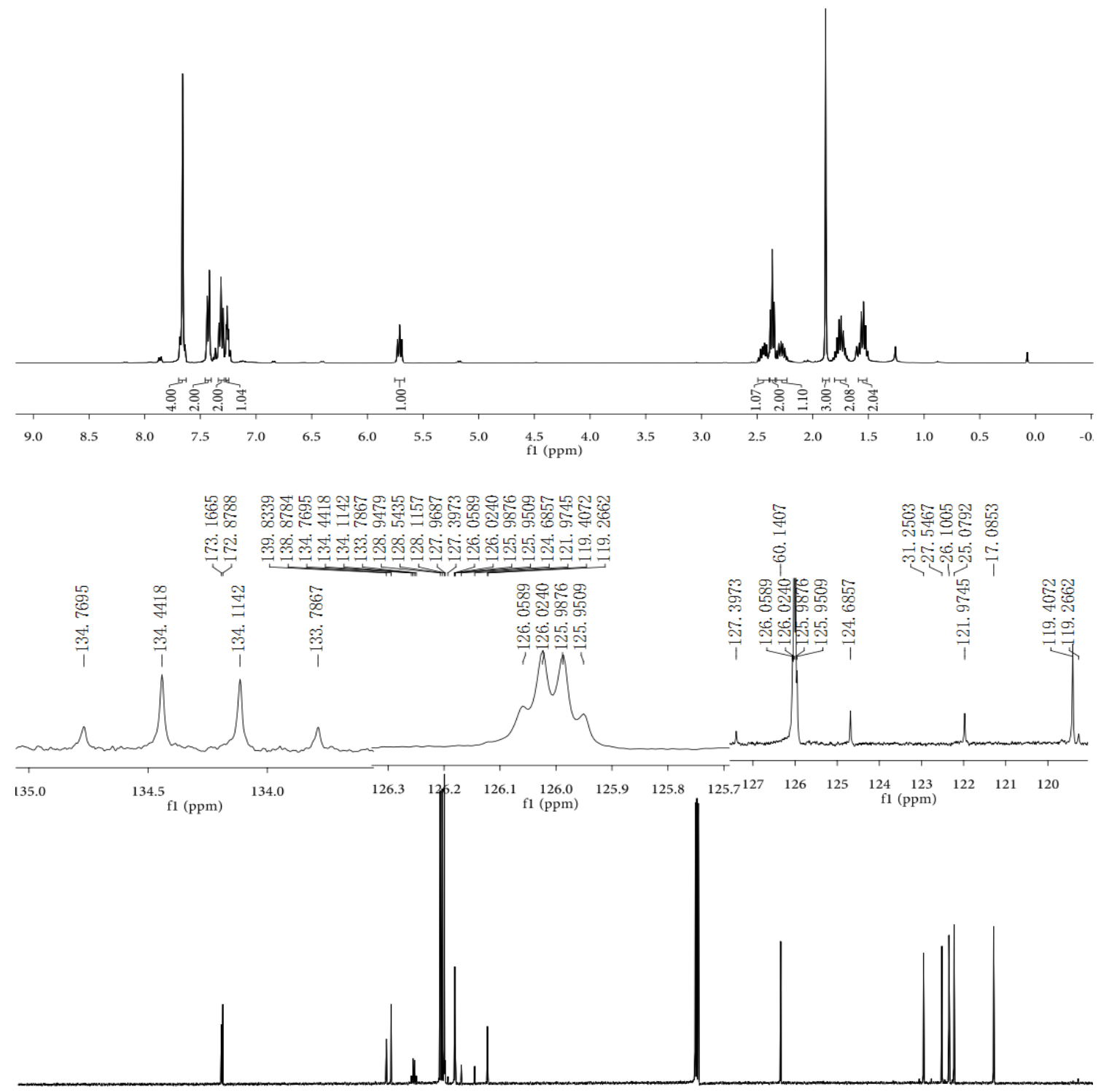

$\begin{array}{rlllllllllllllllllllll}210 & 200 & 190 & 180 & 170 & 160 & 150 & 140 & 130 & 120 & 110 & 100 & 90 & 80 & 70 & 60 & 50 & 40 & 30 & 20 & 10 & 0\end{array}$ 


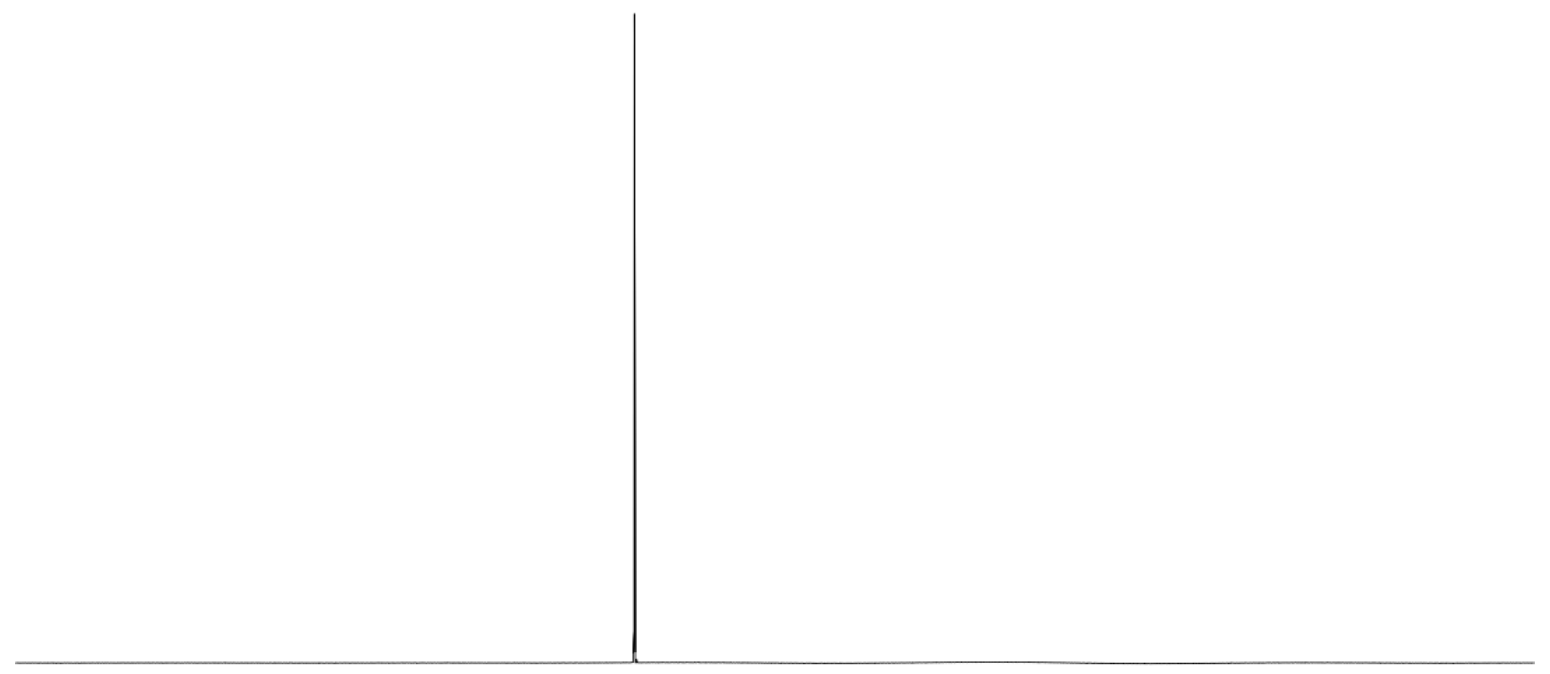

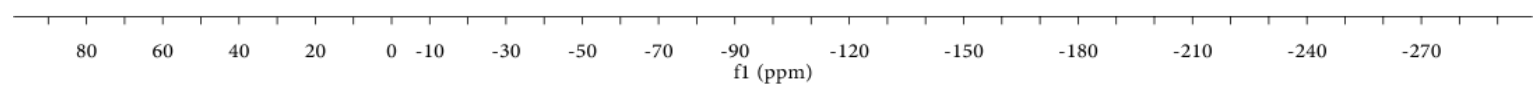


Tert-butyl 5-( $N$-acetyl-4-(trifluoromethyl)benzamido)-2-(cyanomethyl)-5-phenylpentanoate (43):<smiles>CC(=O)N(C(=O)c1ccccc1)C(=O)c1ccc(Br)cc1</smiles>

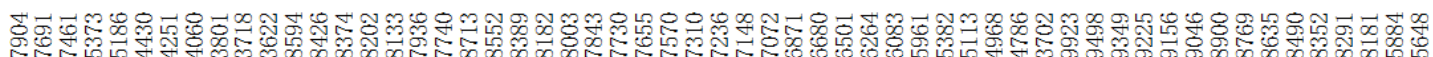

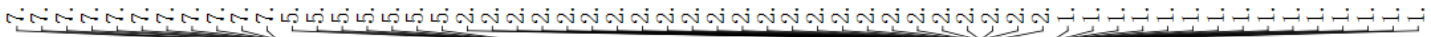

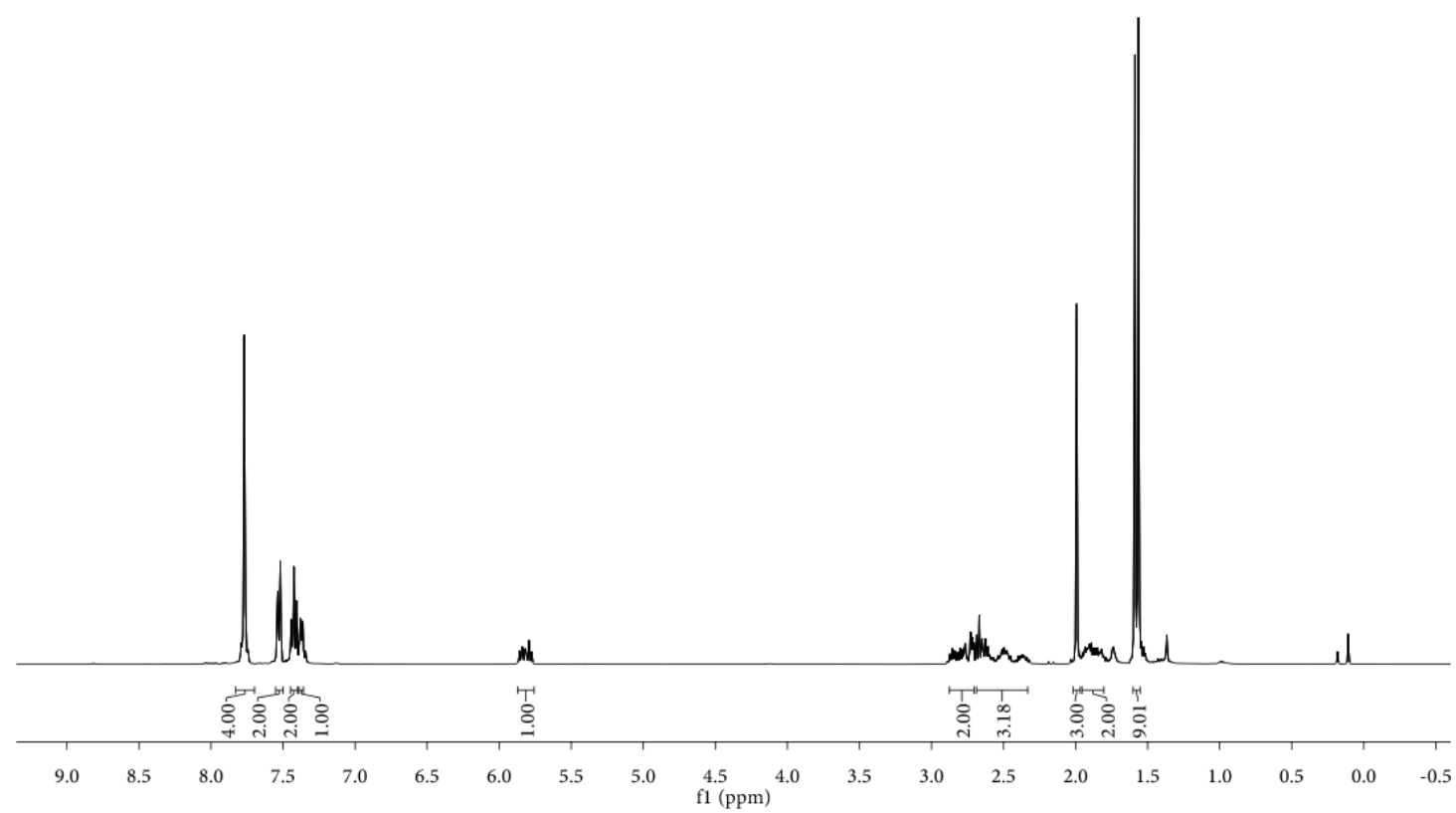

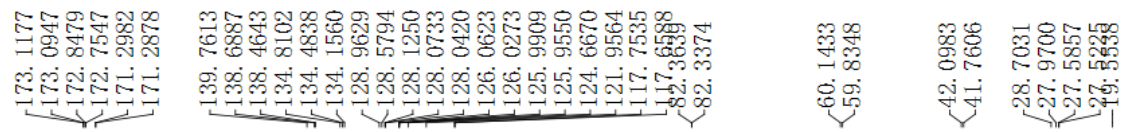

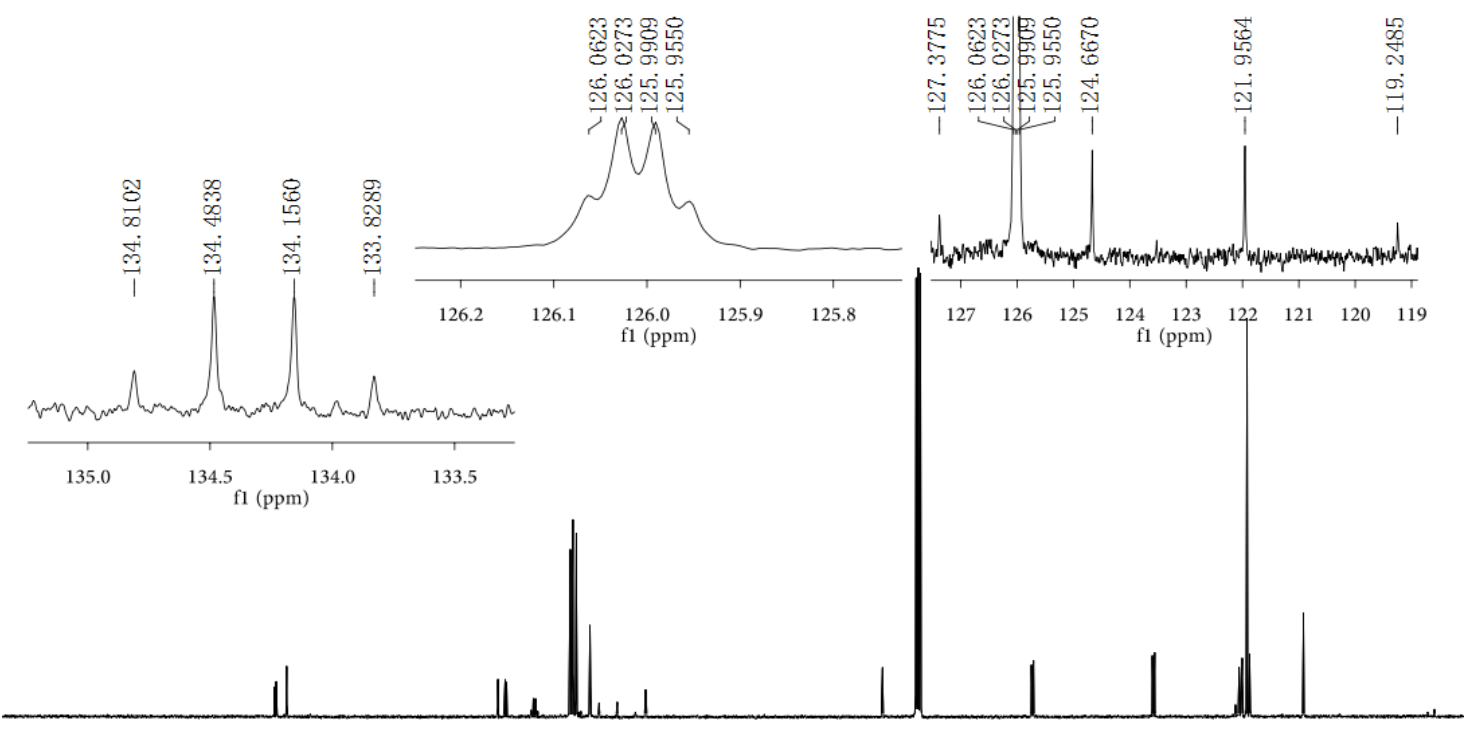

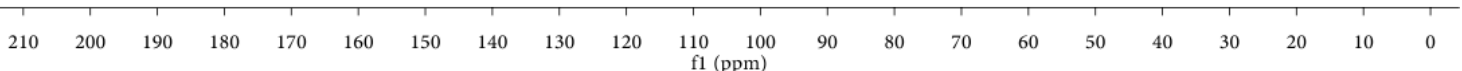




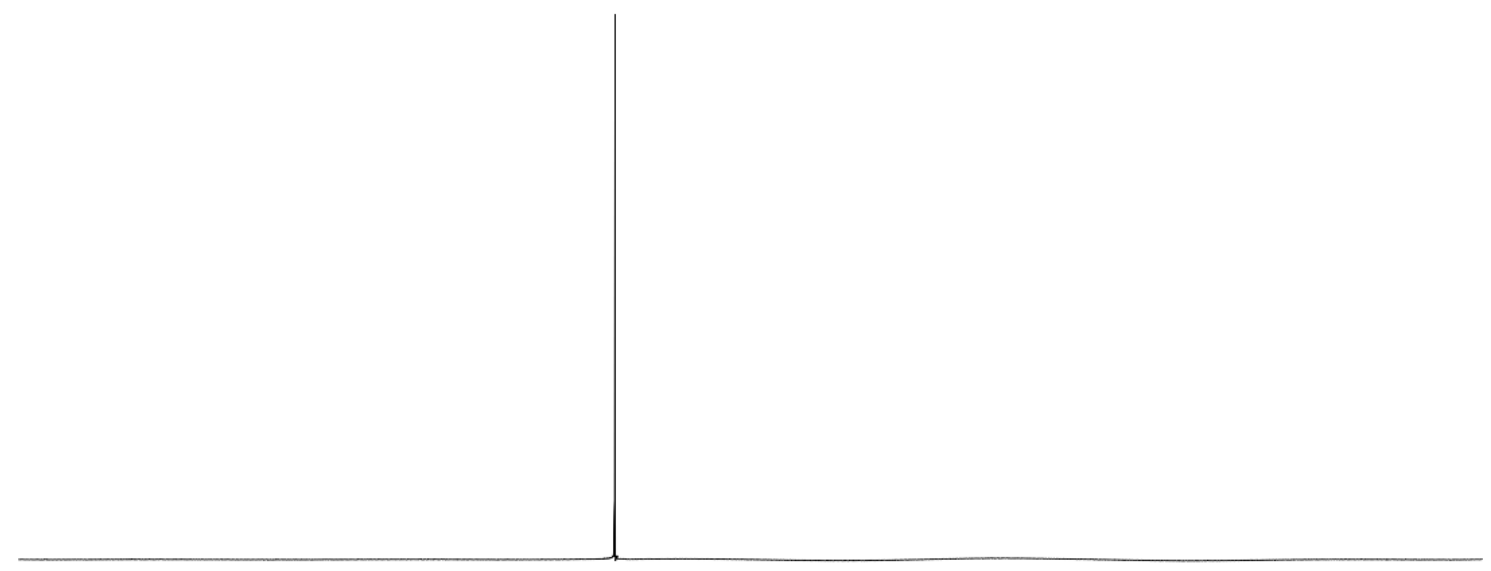

\begin{tabular}{|c|c|c|c|c|c|c|c|c|c|c|c|c|c|c|c|}
\hline 80 & 60 & 40 & 20 & 0 & -10 & -30 & -50 & -70 & $\begin{array}{r}-90 \\
\text { f1 }\end{array}$ & -120 & -150 & -180 & -210 & -240 & -270 \\
\hline
\end{tabular}


Benzyl (5-( $N$-acetyl-4-(trifluoromethyl)benzamido)-1-cyano-5-phenylpentan-2-yl)carbamate (44):<smiles>CC(=O)N(CCC(C)N)C(CCCN)(c1ccccc1)c1ccc(Br)cc1</smiles>

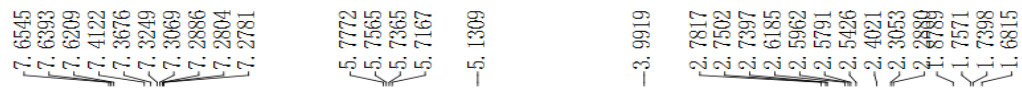
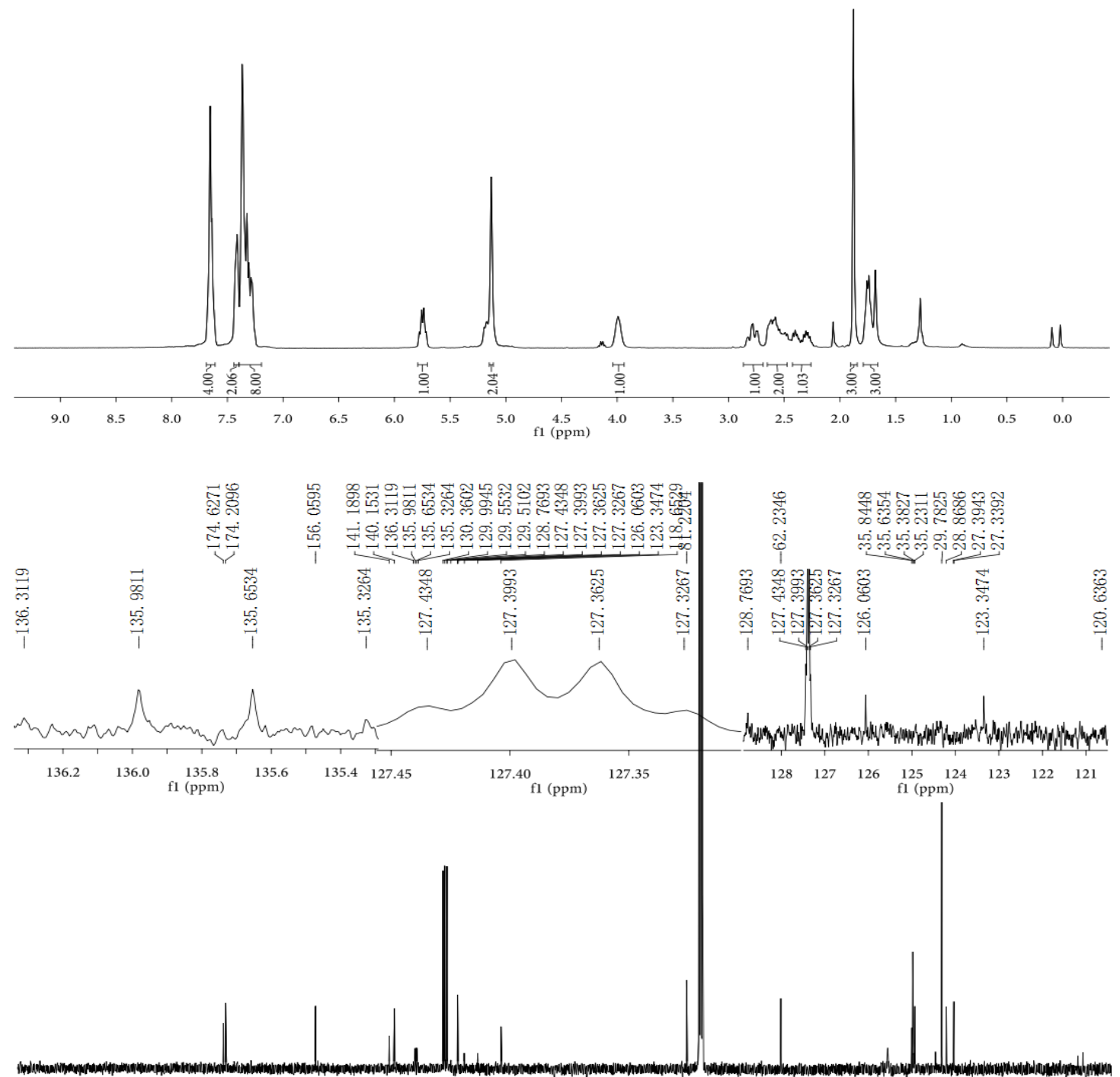

$\begin{array}{llllllllllllllllllllll}210 & 200 & 190 & 180 & 170 & 160 & 150 & 140 & 130 & 120 & 110 & 100 & 90 & 80 & 70 & 60 & 50 & 40 & 30 & 20 & 10 & 0\end{array}$ 


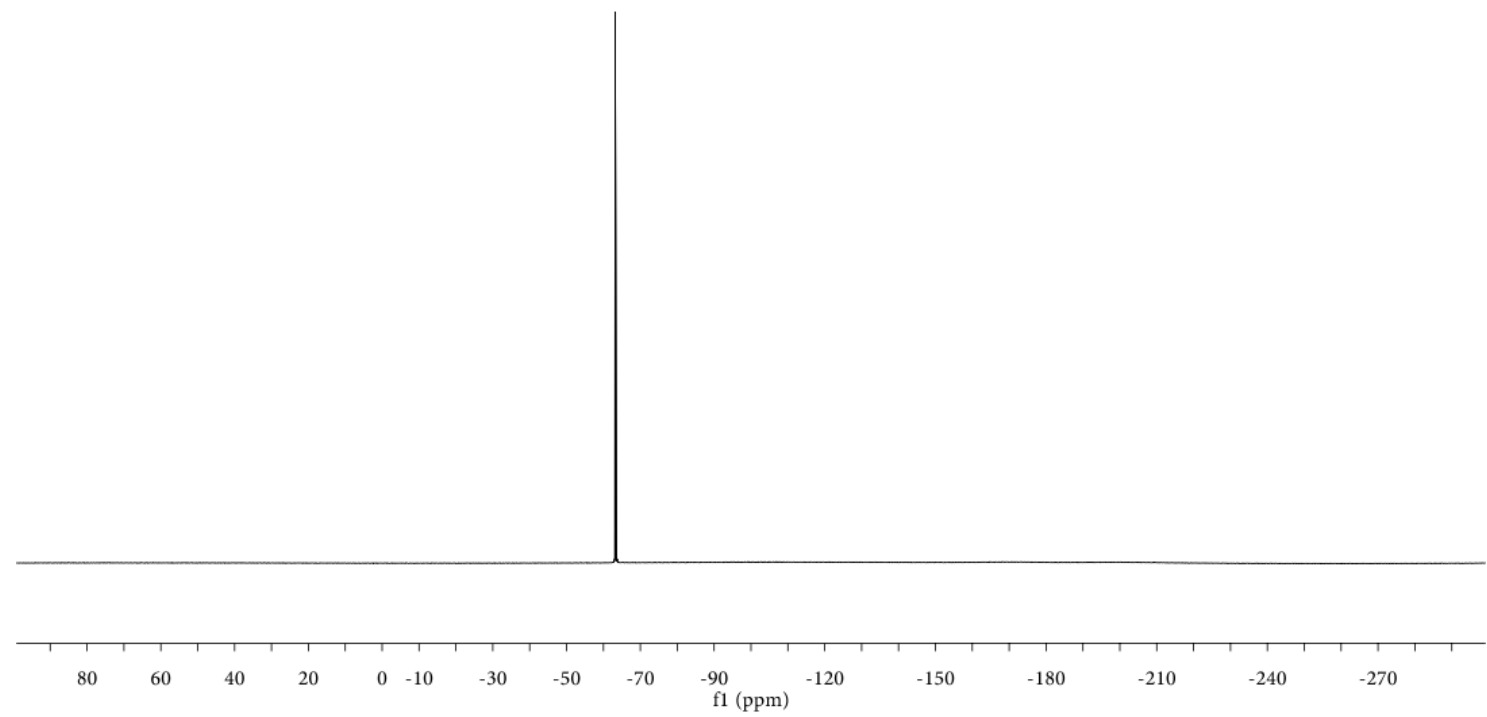


Tert-butyl 4-(3-( $N$-acetyl-4-(trifluoromethyl)benzamido)-3-phenylpropyl)-4(cyanomethyl)piperidine-1-carboxylate (45):<smiles>CCC1(CC(c2ccccc2)N(C(C)=O)C(=O)c2ccc(C)cc2)CCNC1</smiles>
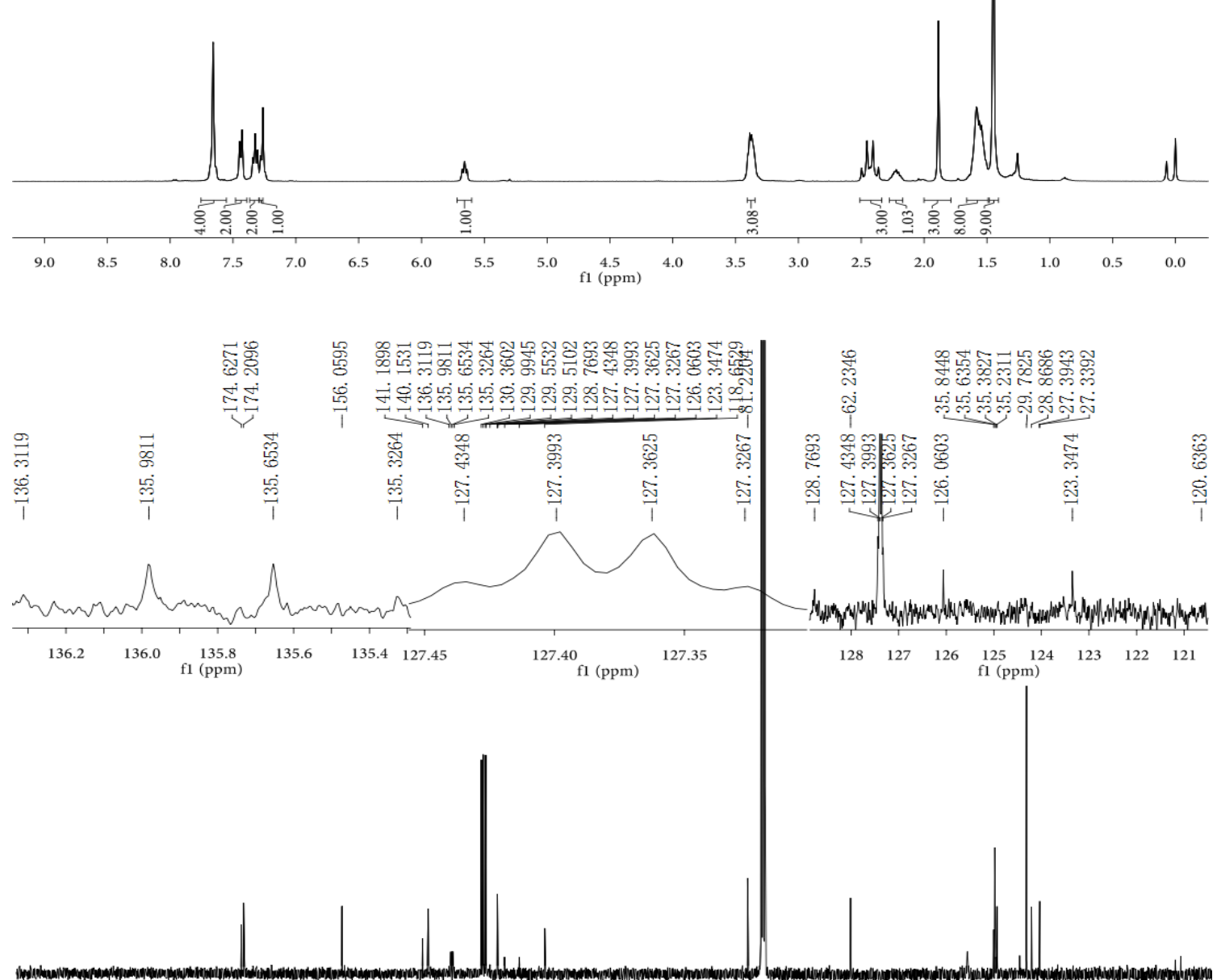

$\begin{array}{lllllllllllllllllllll}210 & 200 & 190 & 180 & 170 & 160 & 150 & 140 & 130 & 120 & \begin{array}{c}110 \\ \mathrm{f} 1(\mathrm{ppm})\end{array} & 90 & 80 & 70 & 60 & 50 & 40 & 30 & 20 & 10 & 0\end{array}$ 


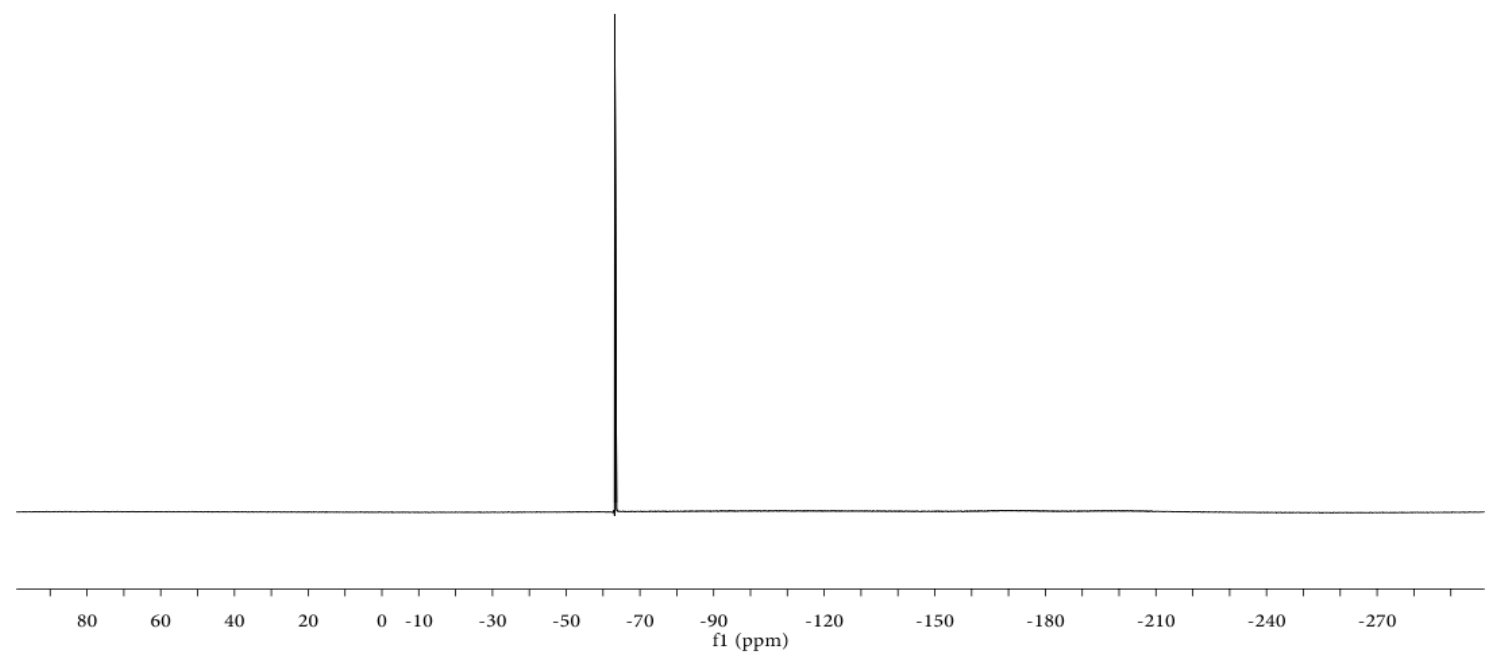


$N$-acetyl- $N$-(2-(2-(cyanomethyl)cyclopent-3-en-1-yl)-1-phenylethyl)-4-

(trifluoromethyl)benzamide (46):<smiles>CCC1(CC(C(C)=O)(c2ccccc2)N(C(C)=O)C(=O)c2ccc(Br)cc2)CCC1</smiles>

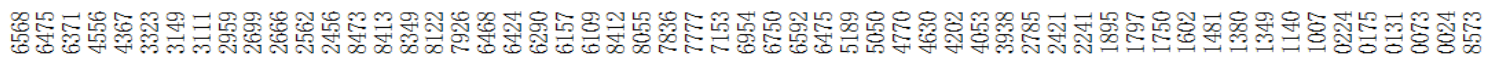

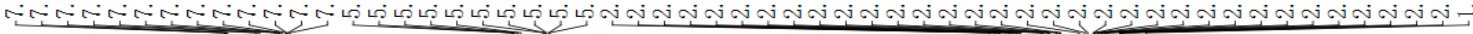

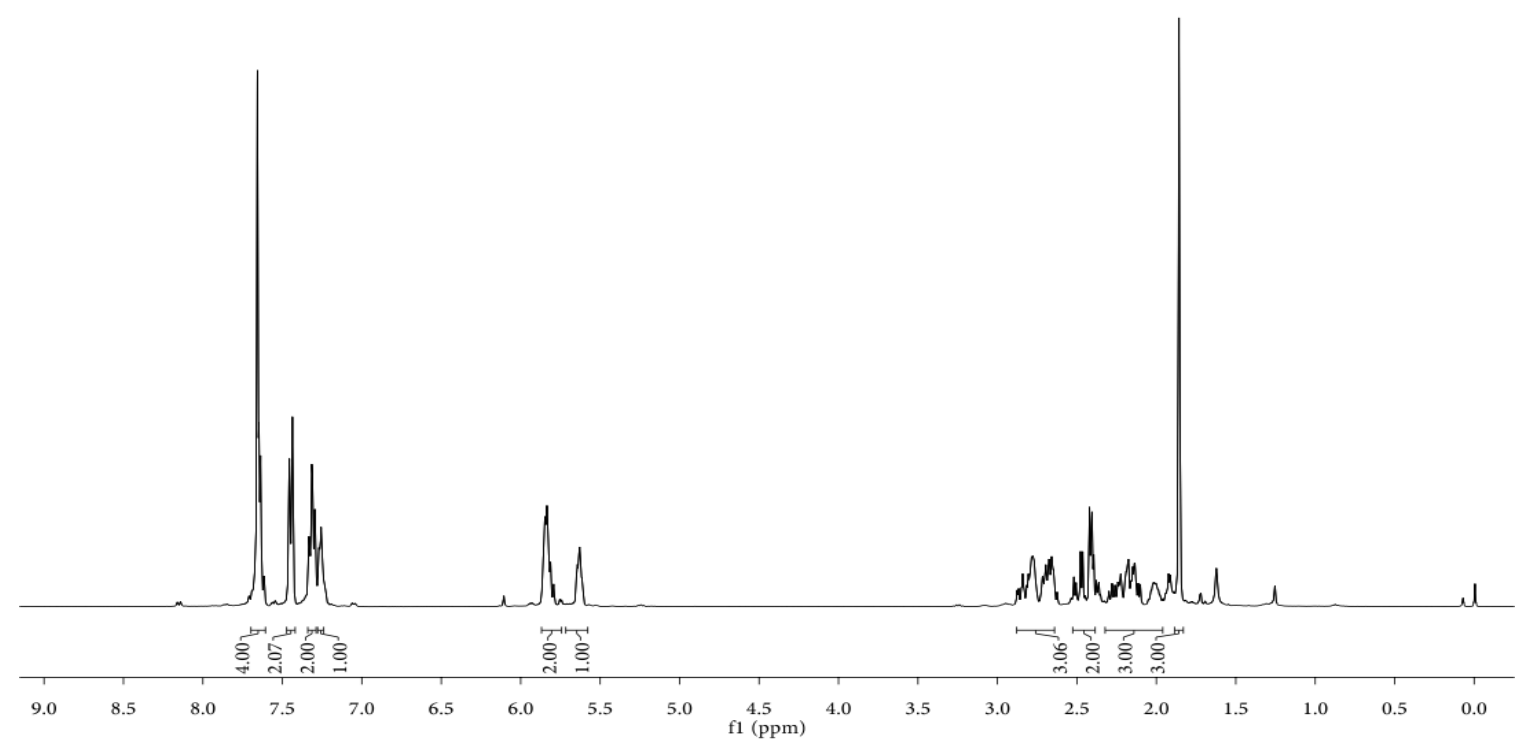

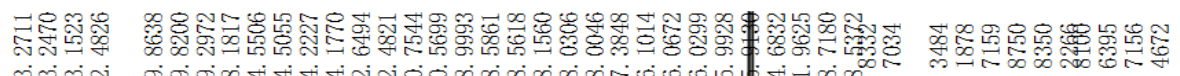

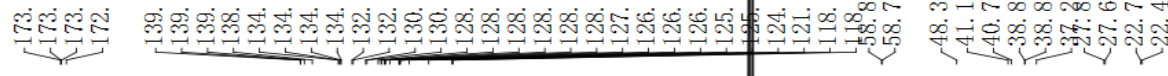

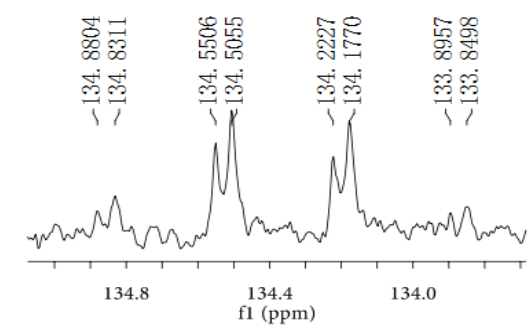

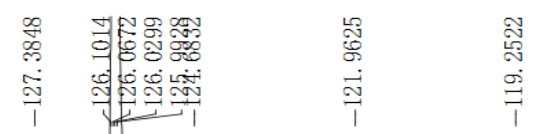




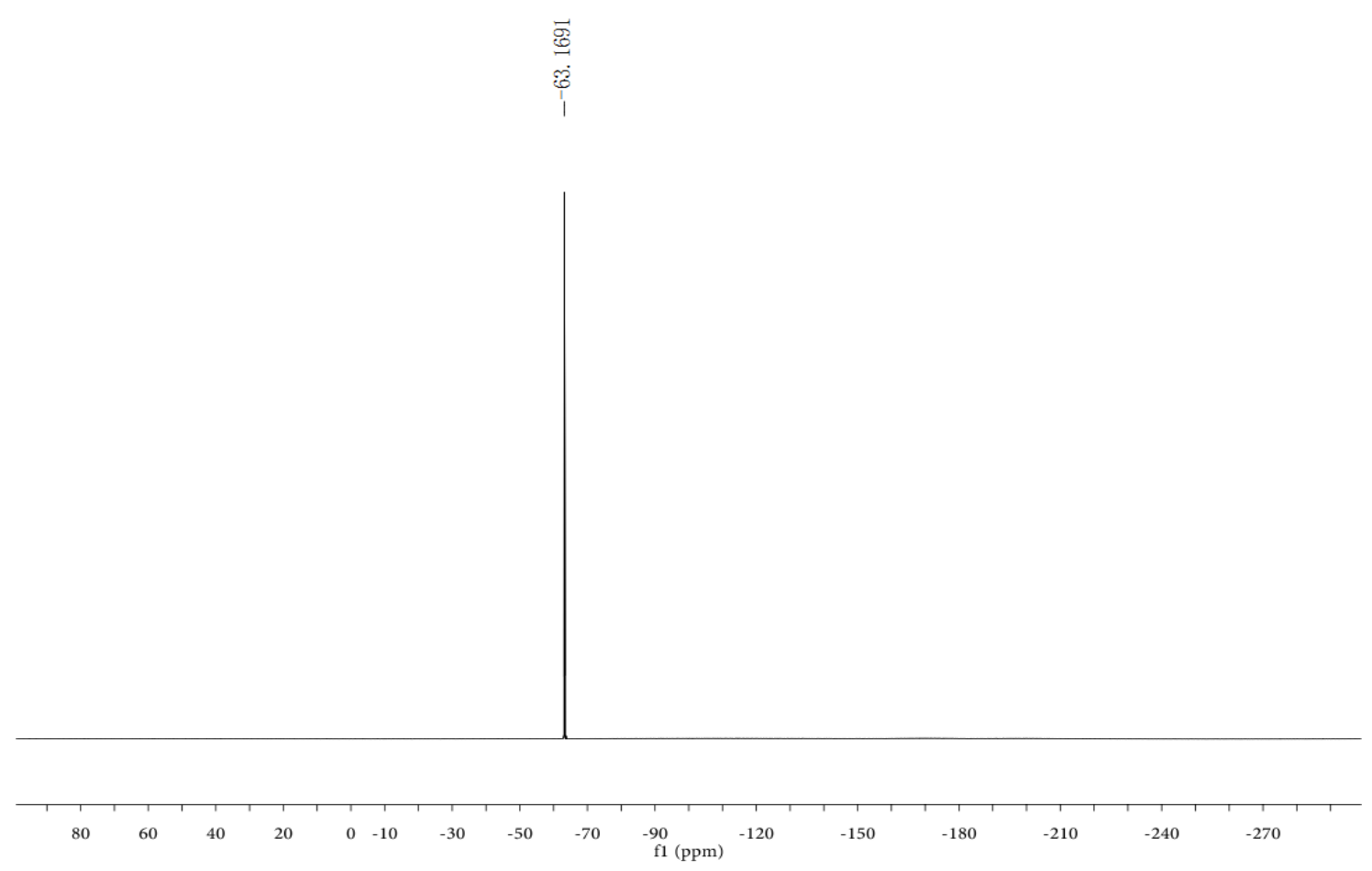




\section{$N$-acetyl- $N$-(3-(2-cyanophenyl)-1-phenylpropyl)-4-(trifluoromethyl)benzamide (47):}<smiles>CC(=O)C(CCc1ccccc1N)(c1ccccc1)c1ccc(Br)cc1</smiles>
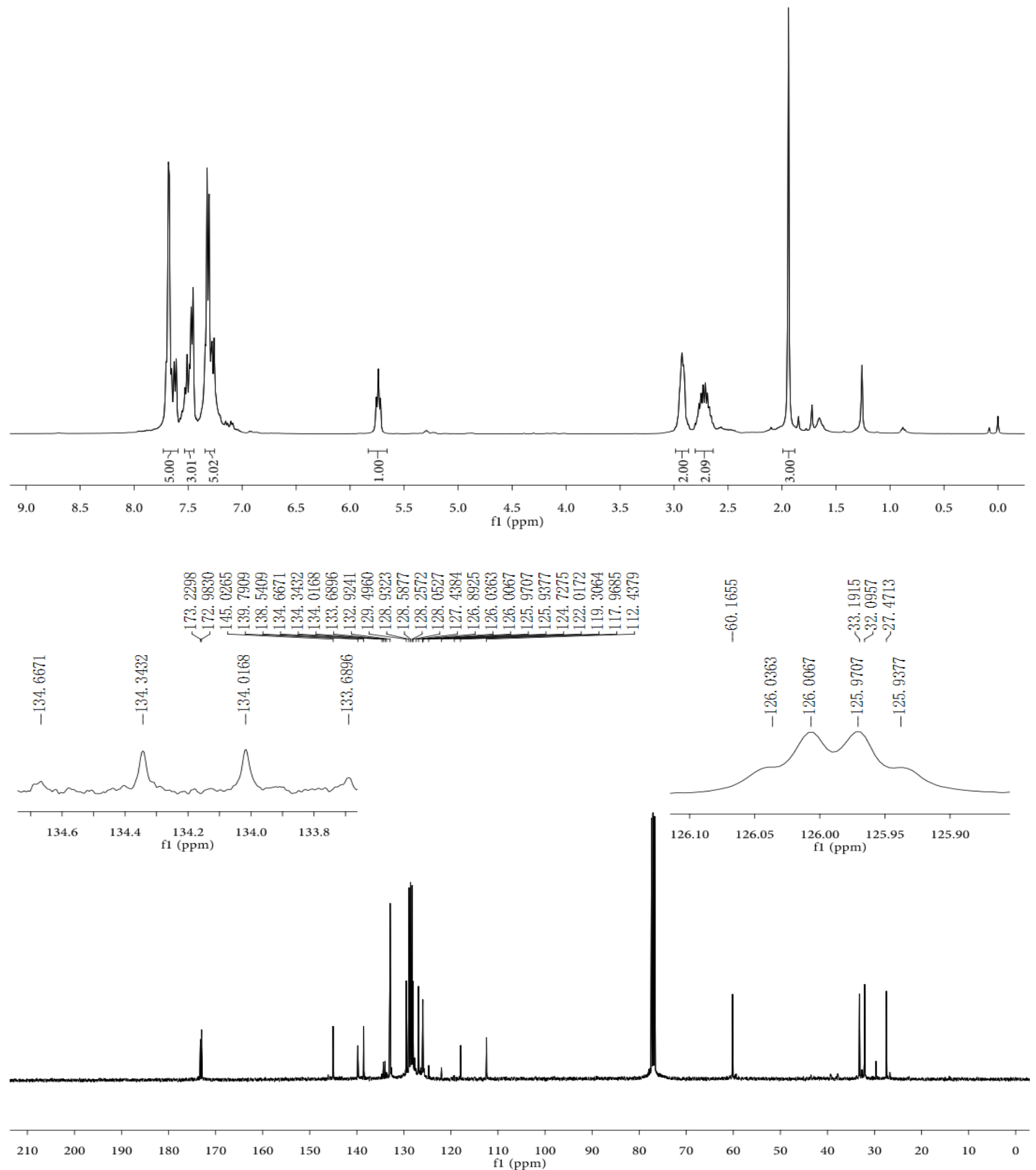

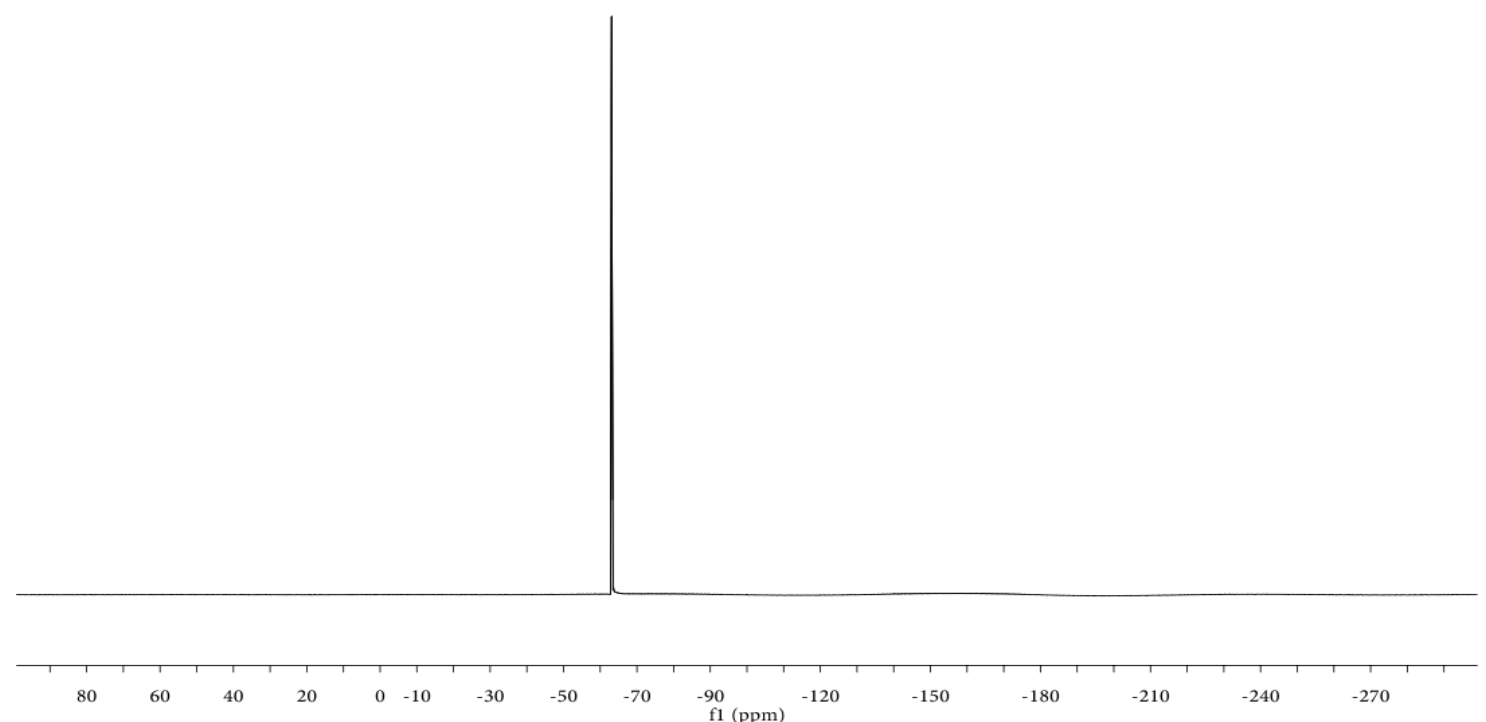
$N$-acetyl- $N$-(6-cyano-3,3-dimethyl-1-phenylhexyl)-4-(trifluoromethyl)benzamide (48):<smiles>CC(=O)N(c1ccc(Br)cc1)C(CC(C)(C)CCO)(C(C)=O)c1ccccc1</smiles>

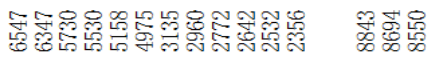

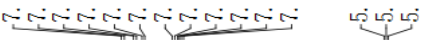

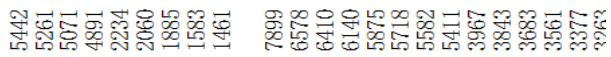
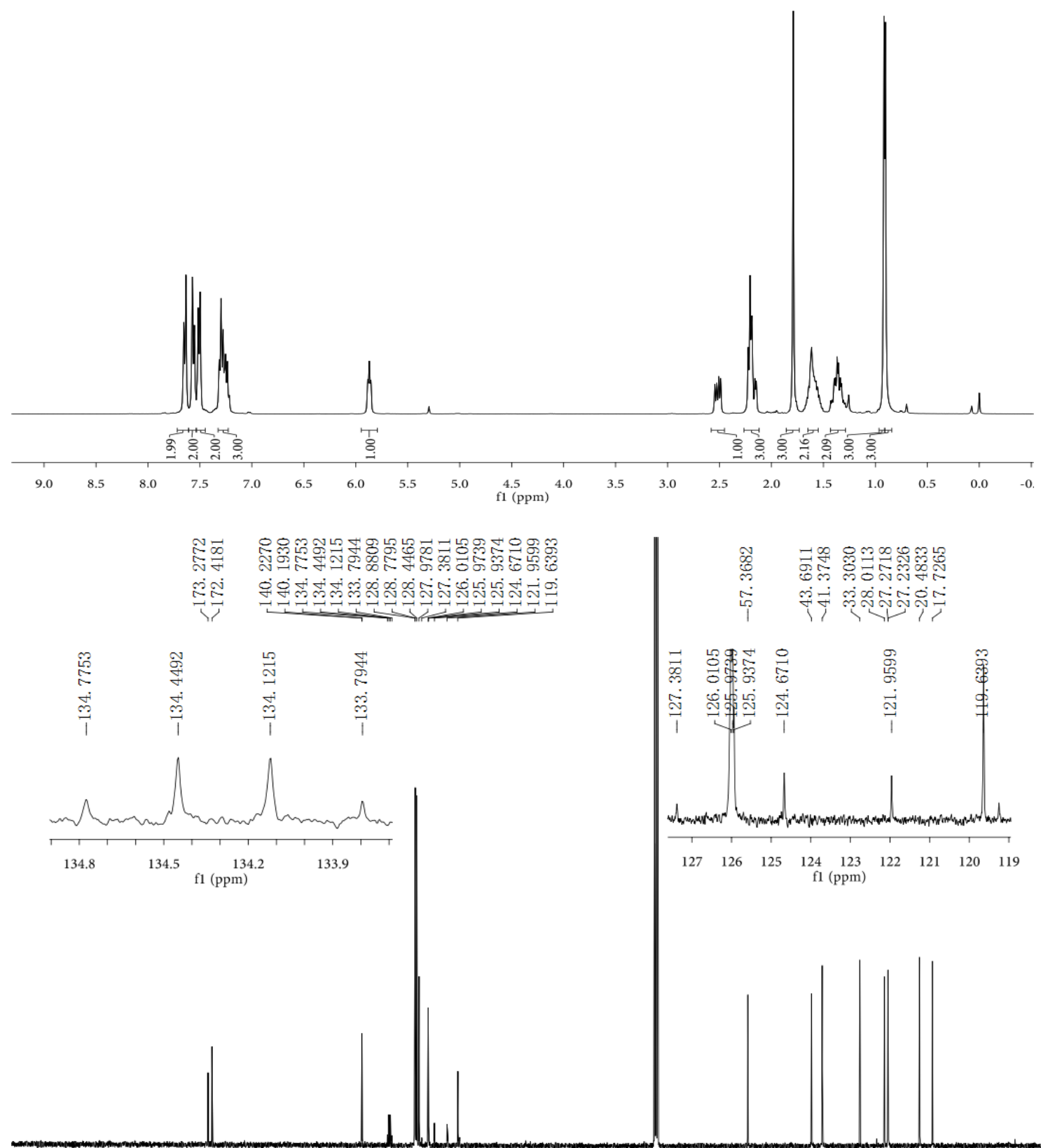

$\begin{array}{llllllllllllllllllllll}210 & 200 & 190 & 180 & 170 & 160 & 150 & 140 & 130 & 120 & 110 & 100 & 90 & 80 & 70 & 60 & 50 & 40 & 30 & 20 & 10 & 0\end{array}$ 


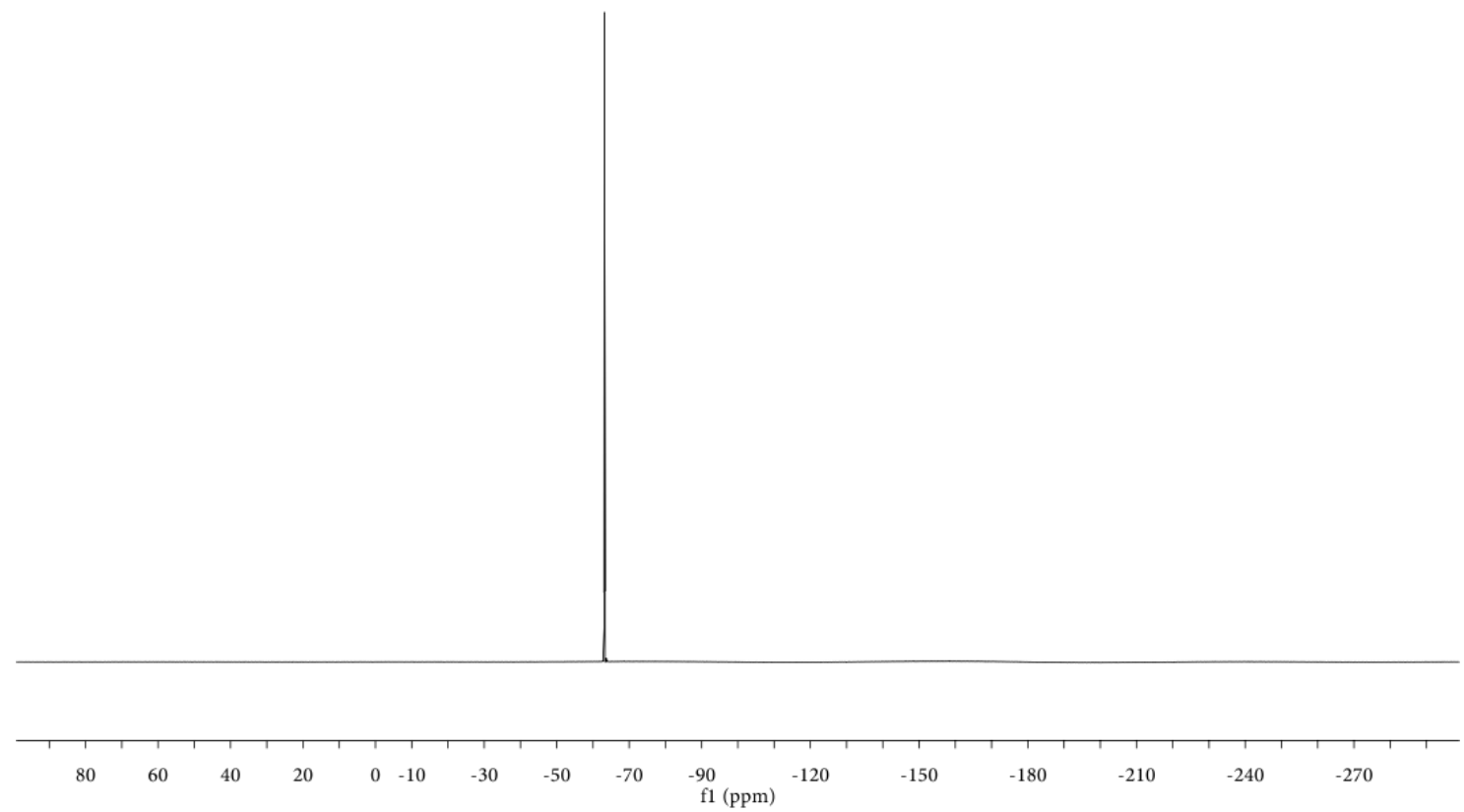


$N$-acetyl- $N$-(5-cyano-1-(o-tolyl)pentyl)-4-(trifluoromethyl)benzamide (49):<smiles>CCCCCC(c1ccccc1C)N(C(C)=O)C(=O)c1ccc(C(F)(F)F)cc1</smiles>

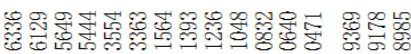

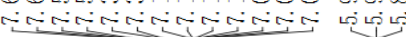

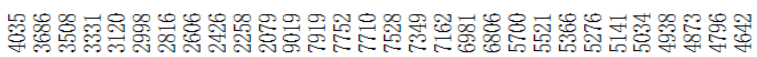

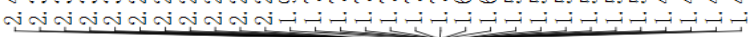
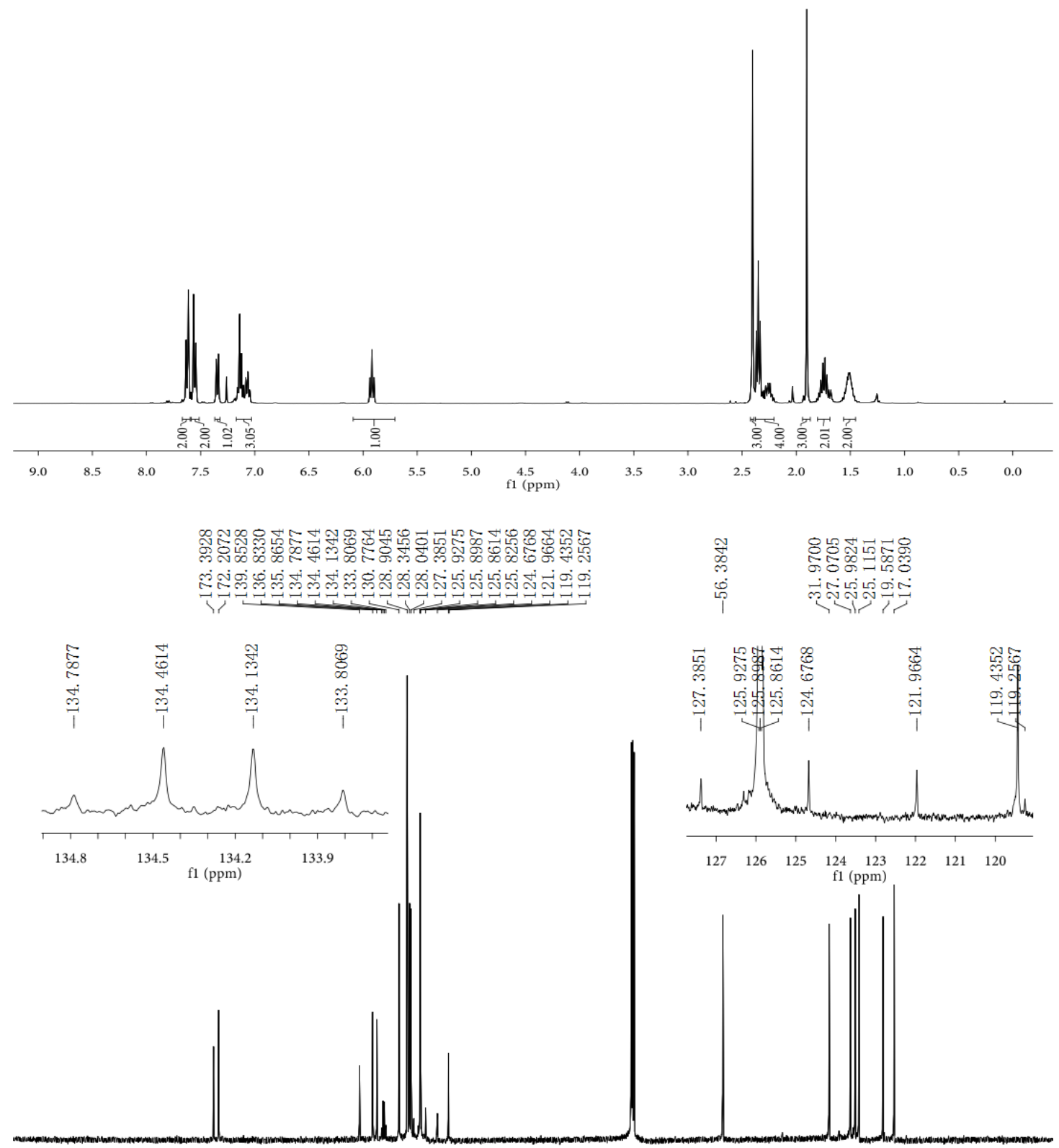

$\begin{array}{lllllllllllllllllllllll}210 & 200 & 190 & 180 & 170 & 160 & 150 & 140 & 130 & 120 & 110 & \begin{array}{c}100 \\ \mathrm{f} 1(\mathrm{ppm})\end{array} & 90 & 80 & 70 & 60 & 50 & 40 & 30 & 20 & 10 & 0 & -10\end{array}$ 


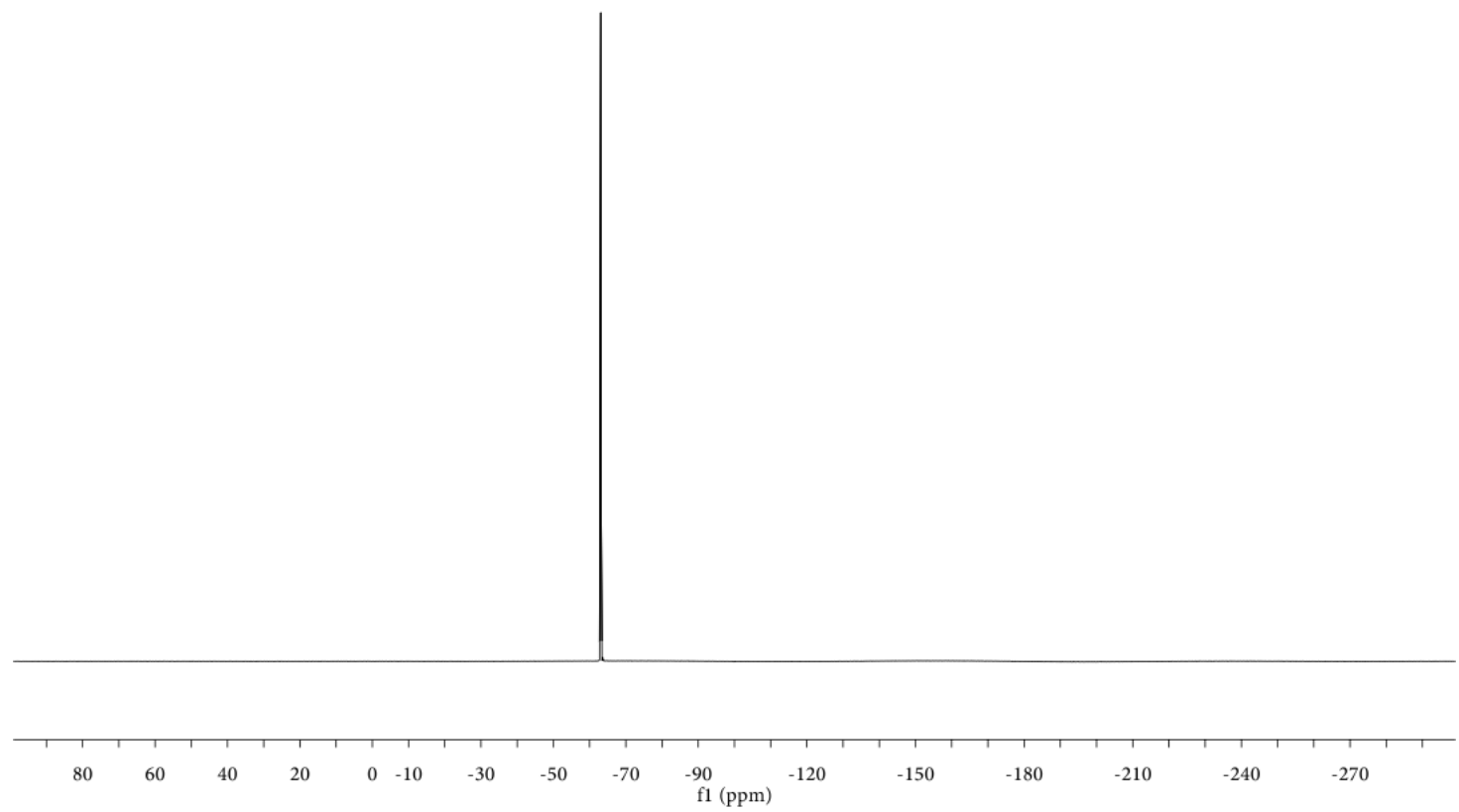


$N$-acetyl- $N$-(5-cyano-1-(m-tolyl)pentyl)-4-(trifluoromethyl)benzamide (50):<smiles>CCCCCC(c1cccc(C)c1)N(C(C)=O)C(=O)c1ccc(C(F)(F)F)cc1</smiles>

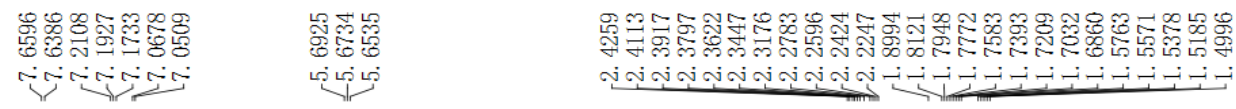
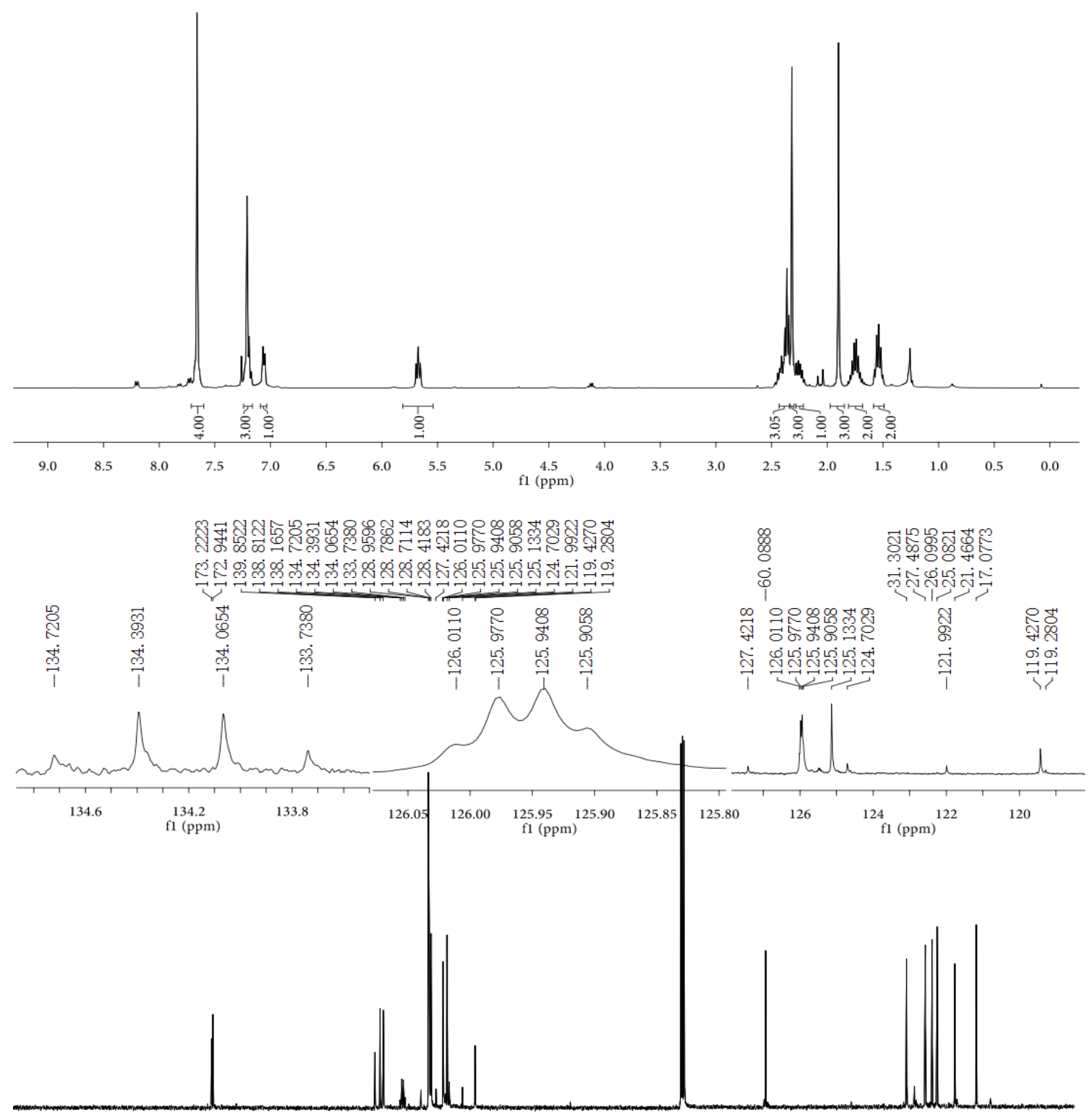

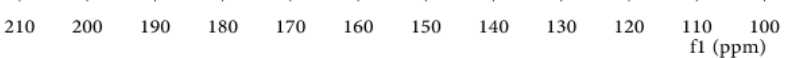




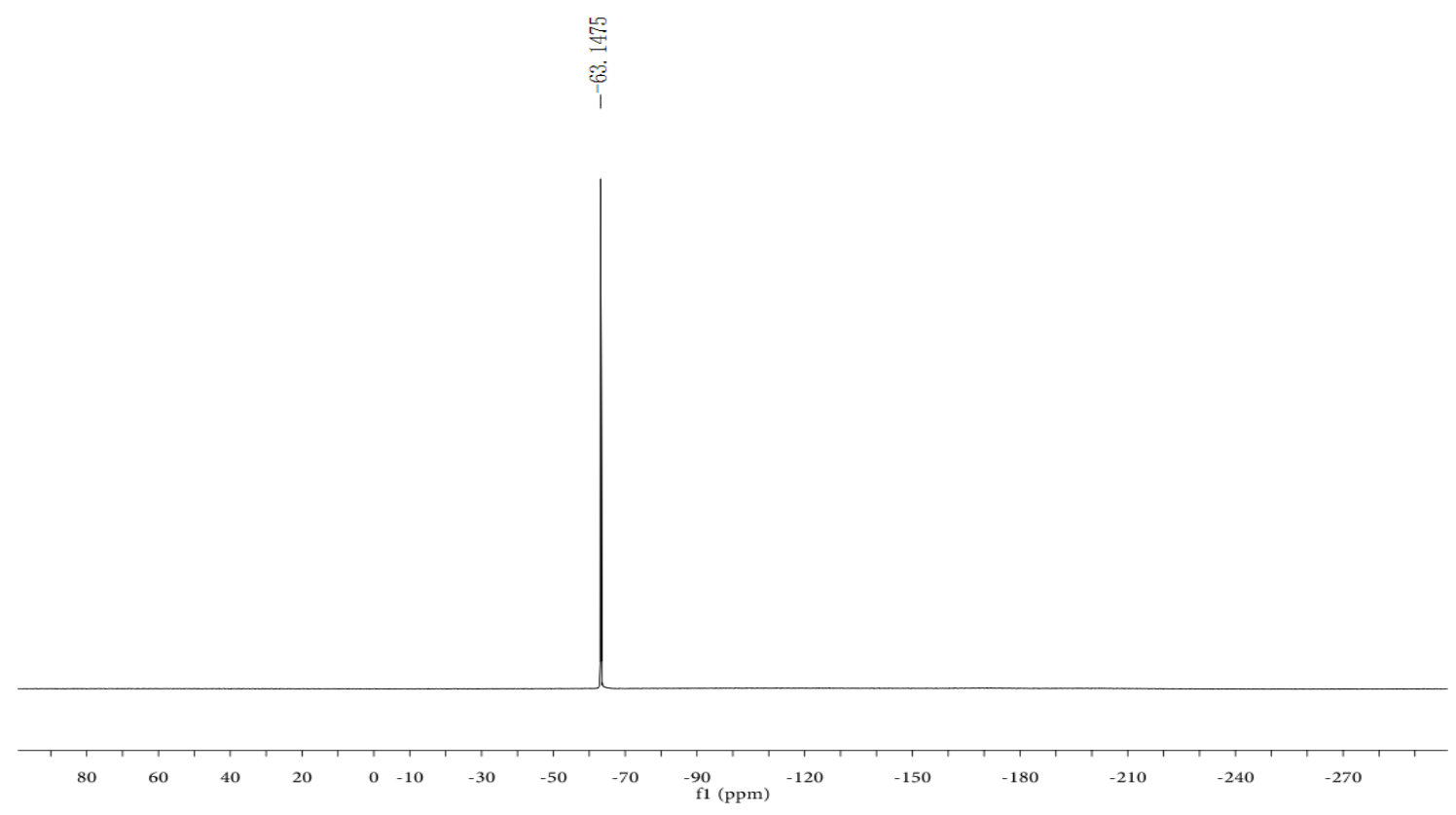


$N$-acetyl- $N$-(5-cyano-1-(p-tolyl)pentyl)-4-(trifluoromethyl)benzamide (51):<smiles>CC(=O)N(C(=O)c1ccc(C)cc1)C(CCCCO)c1ccc(C)cc1</smiles>

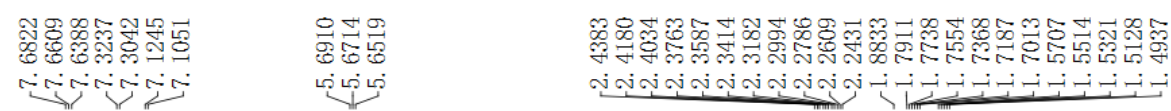

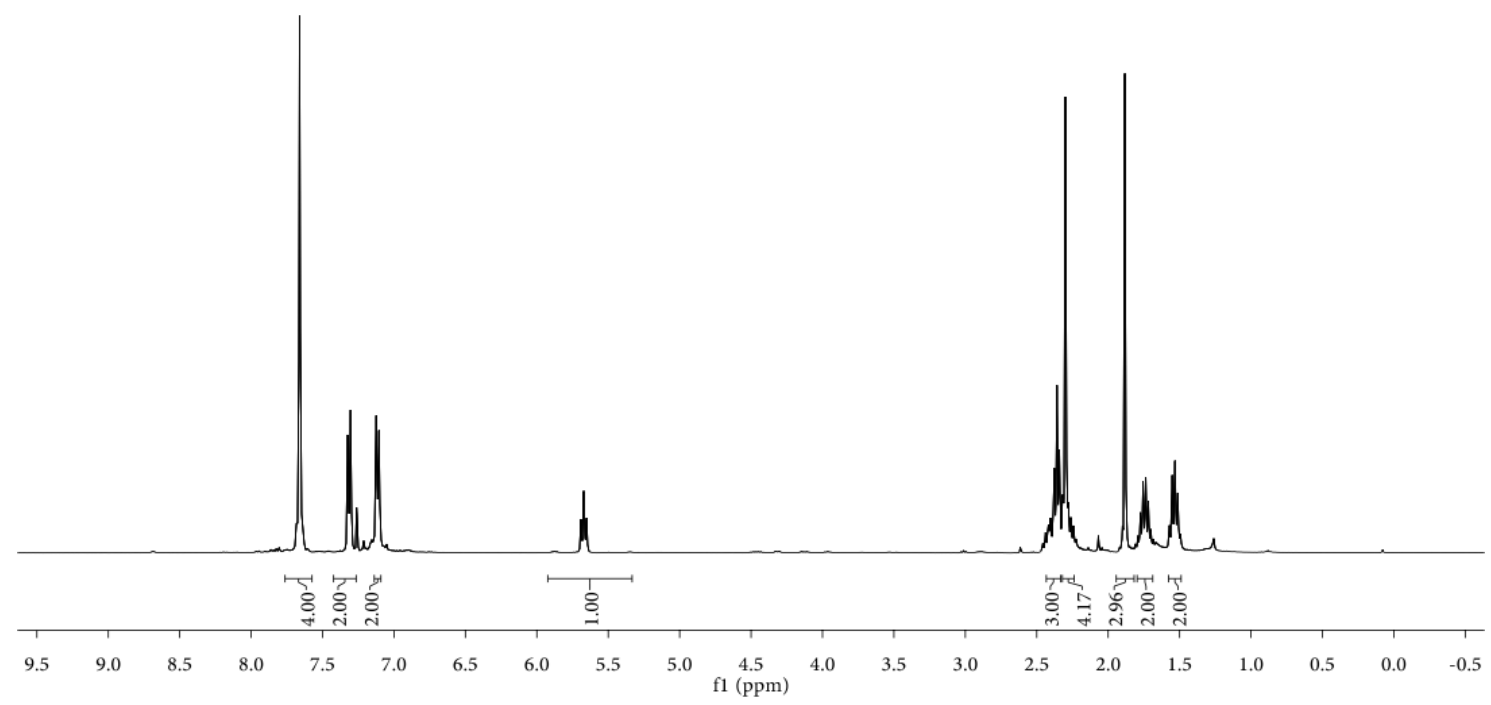

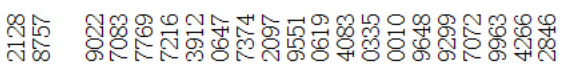

象
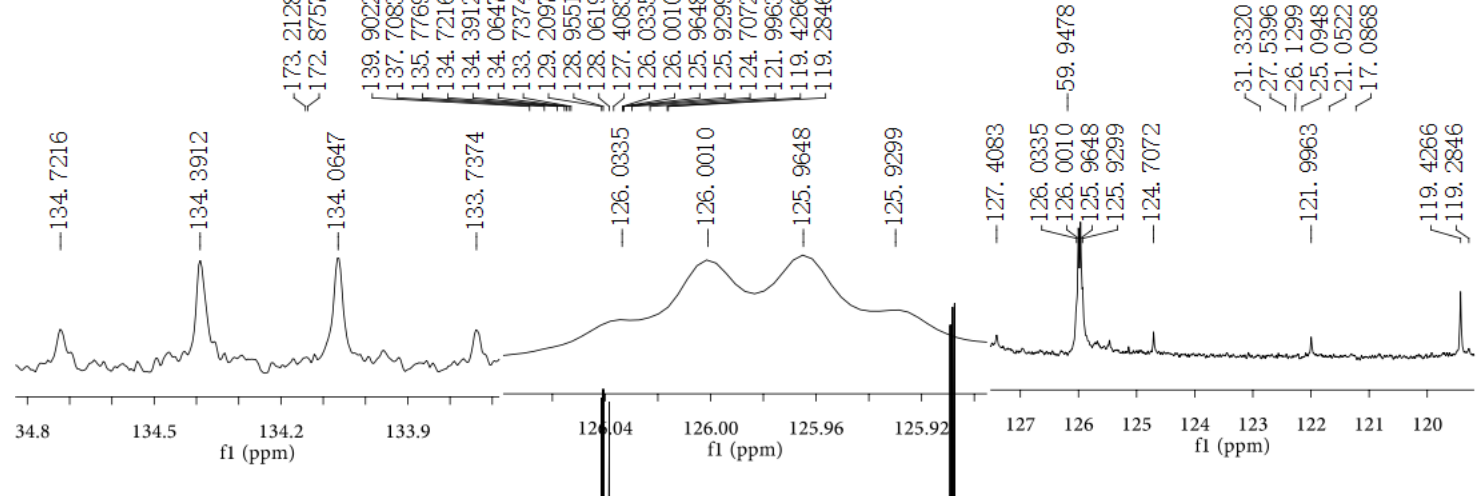

$\begin{array}{lllllllllll}110 & 200 & 190 & 180 & 170 & 160 & 150 & 140 & 130 & 120 & 110 \quad 100 \\ \mathrm{f} 1(\mathrm{ppm})\end{array}$ 


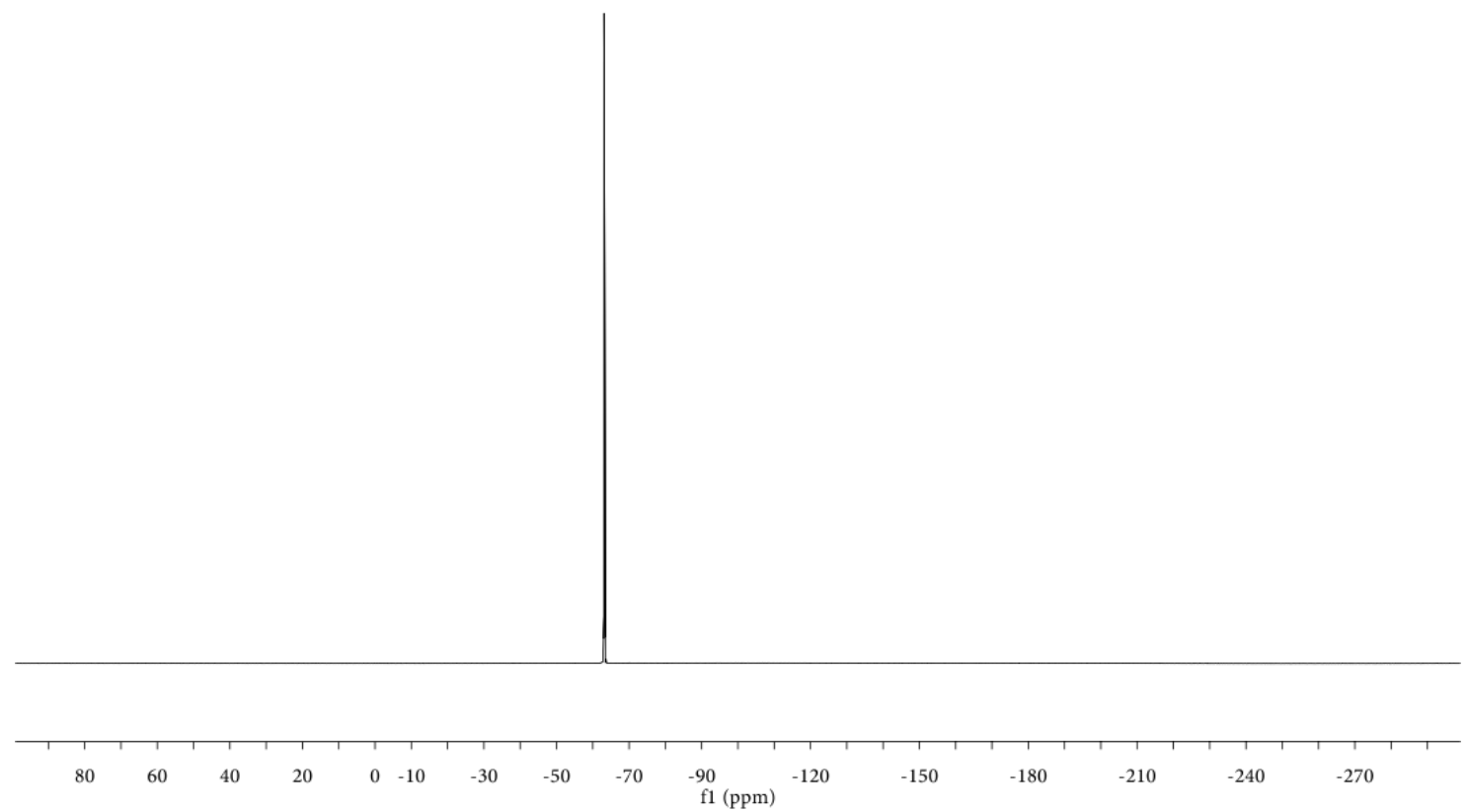


4-(1-( $N$-acetyl-4-(trifluoromethyl)benzamido)-5-cyanopentyl)phenyl acetate (52):<smiles>CCCCC(c1ccc(OC(C)=O)cc1)N(C(C)=O)c1ccc(C)cc1</smiles>

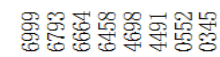

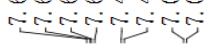

논동용

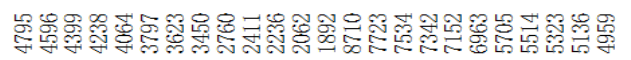

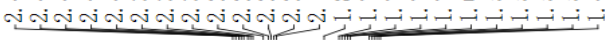
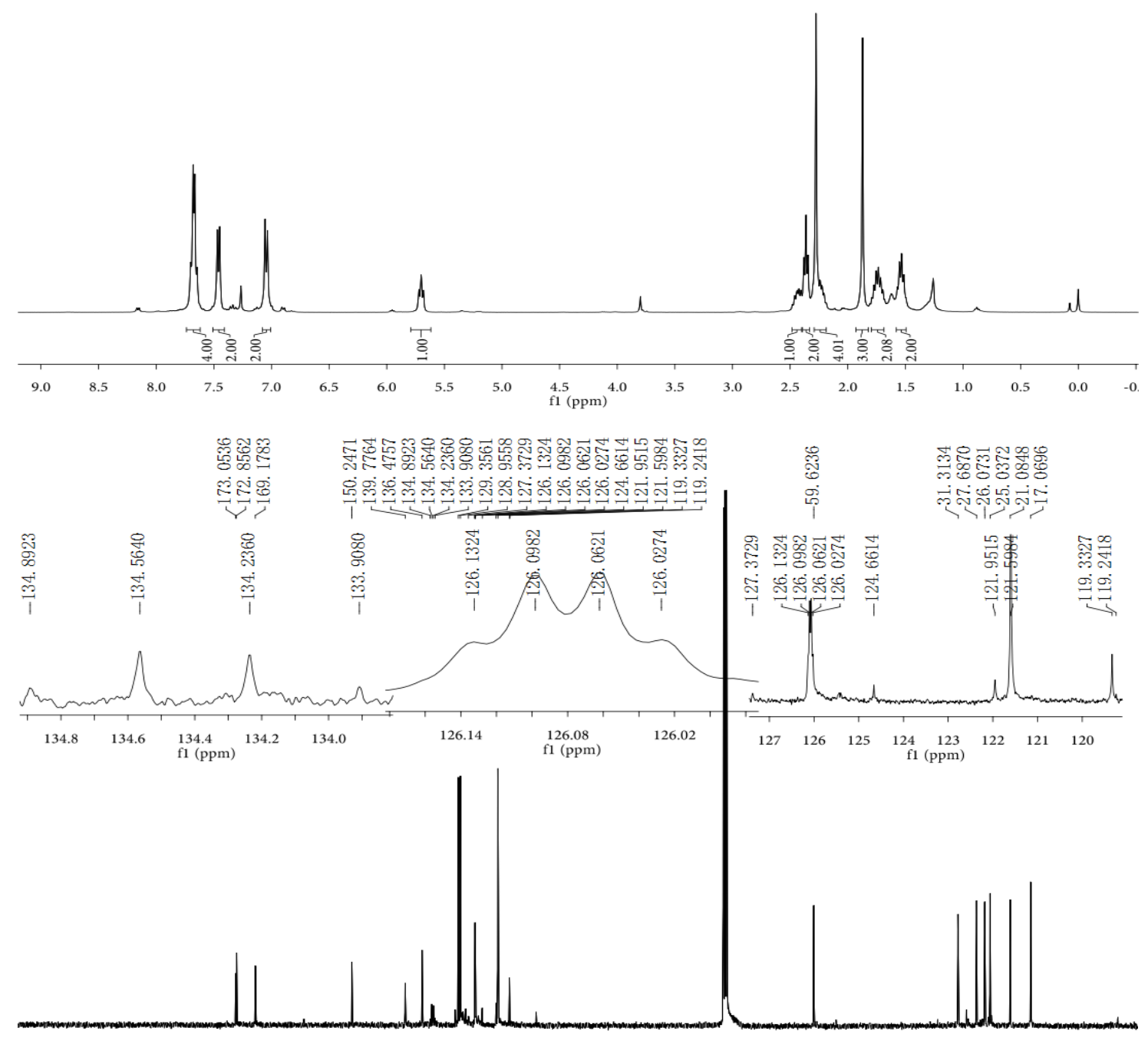

$\begin{array}{lllllllllllllllllllll}210 & 200 & 190 & 180 & 170 & 160 & 150 & 140 & 130 & 120 & \begin{array}{l}110 \\ \mathrm{f} 1(\mathrm{ppm})\end{array} & 90 & 80 & 70 & 60 & 50 & 40 & 30 & 20 & 10 & 0\end{array}$ 


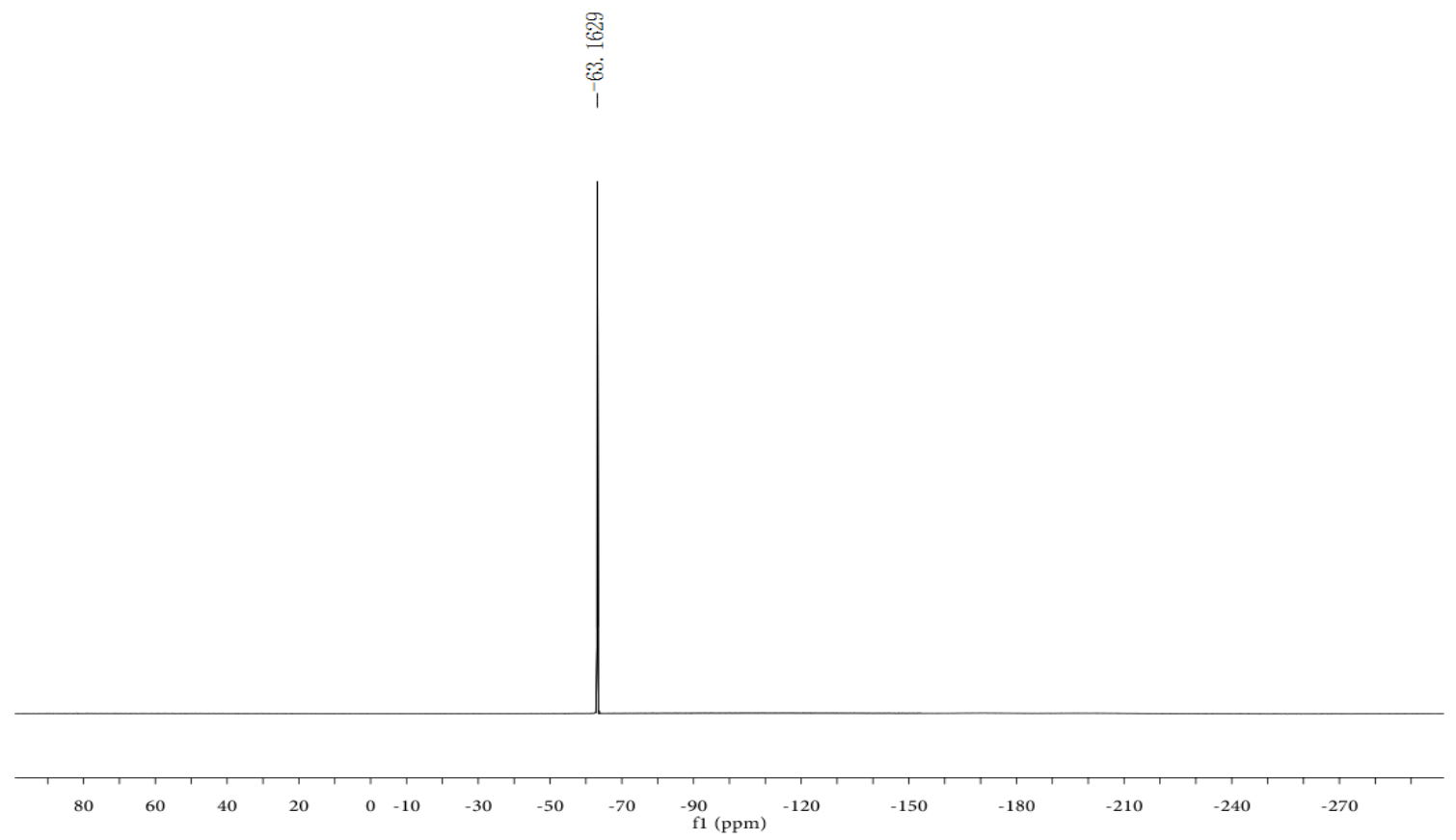


$N$-acetyl- $N$-(5-cyano-1-(4-fluorophenyl)pentyl)-4-(trifluoromethyl)benzamide (53):

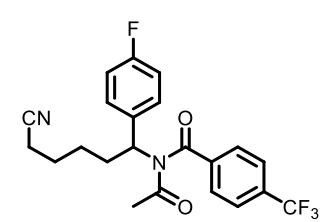

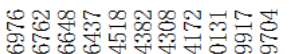

nininimion

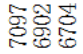

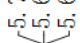

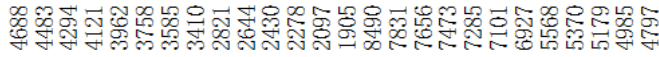

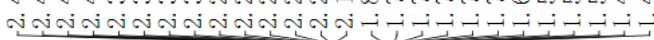

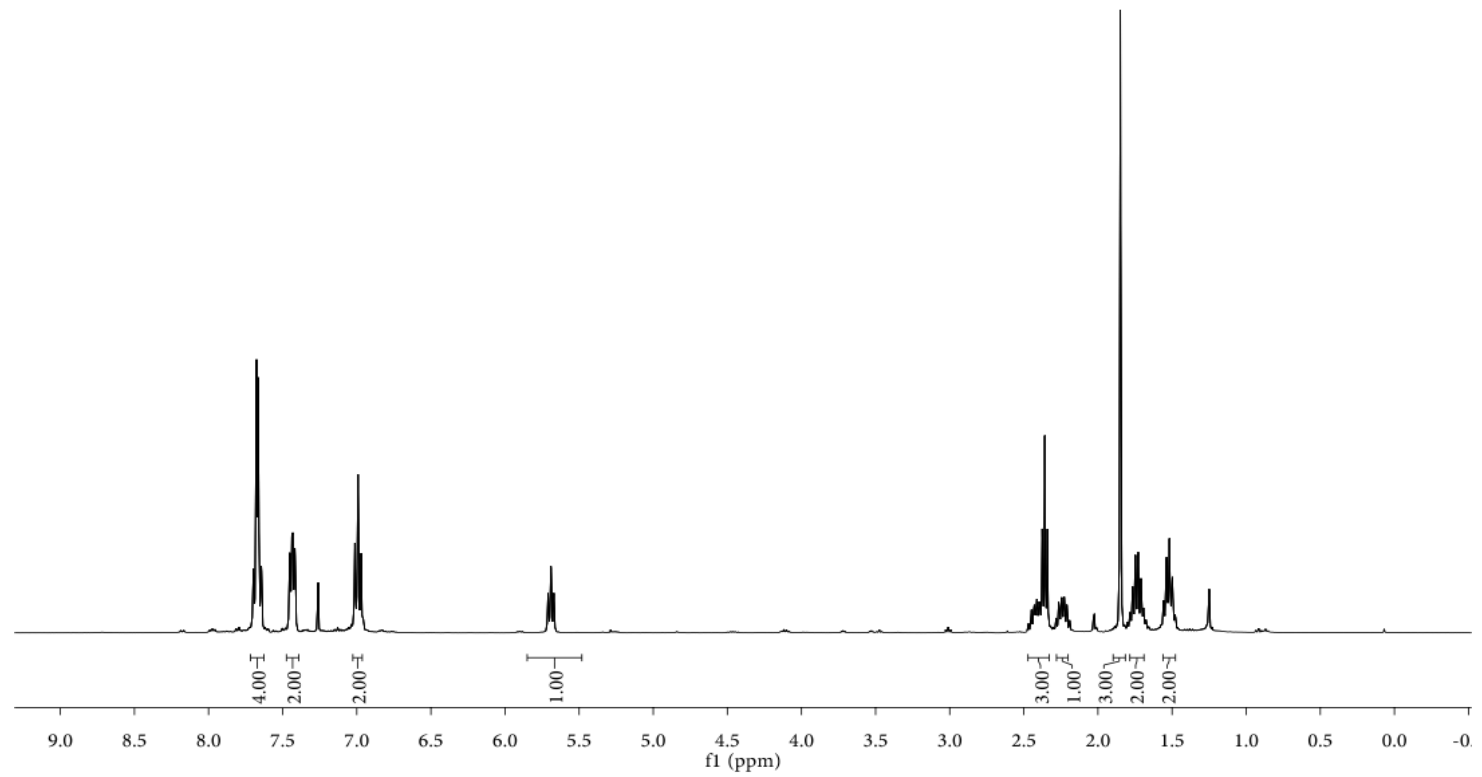

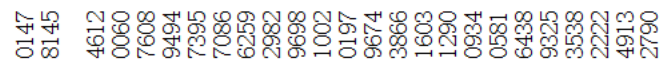

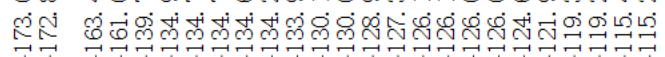

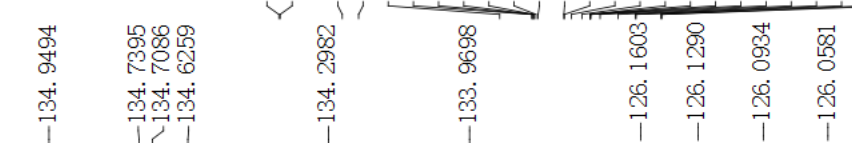

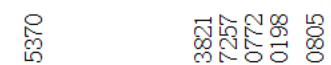

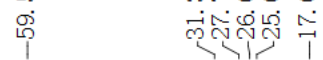

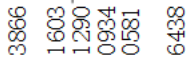

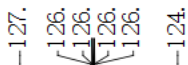

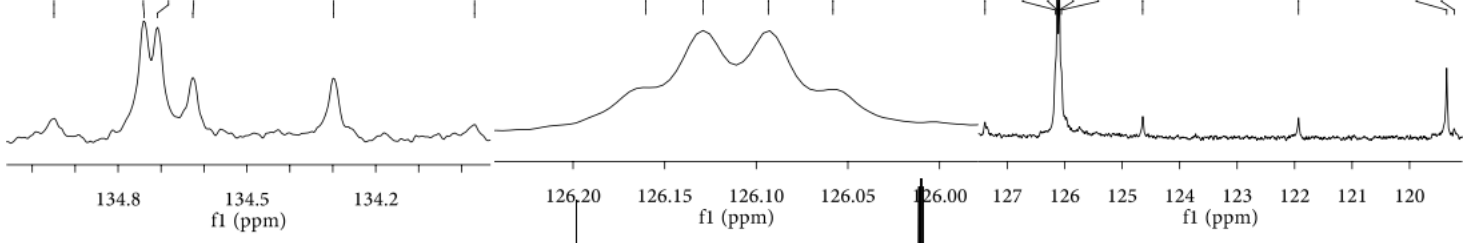

134.2 $126,20 \quad 126.15 \underset{f 1(p p m)}{126.10} \quad 126.05$

$\begin{array}{rrrrr}6.00 & 127 & 126 & 125 & 124 \\ & & & & \text { f1 (ppm) }\end{array}$ 


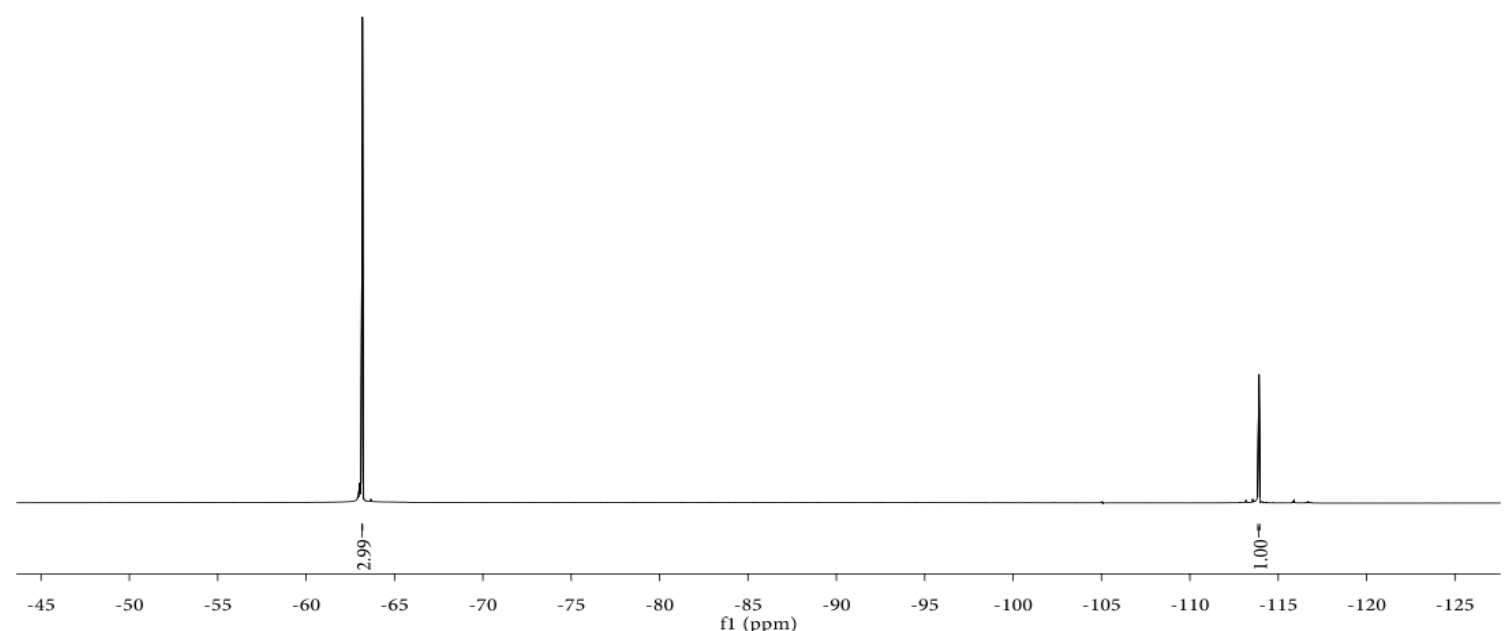


$N$-acetyl- $N$-(1-(4-chlorophenyl)-5-cyanopentyl)-4-(trifluoromethyl)benzamide (54):<smiles>CCCCC(C(=O)O)(c1ccc(Cl)cc1)N(C(C)=O)C(=O)c1ccc(Cl)cc1</smiles>

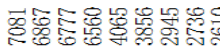
risicicis
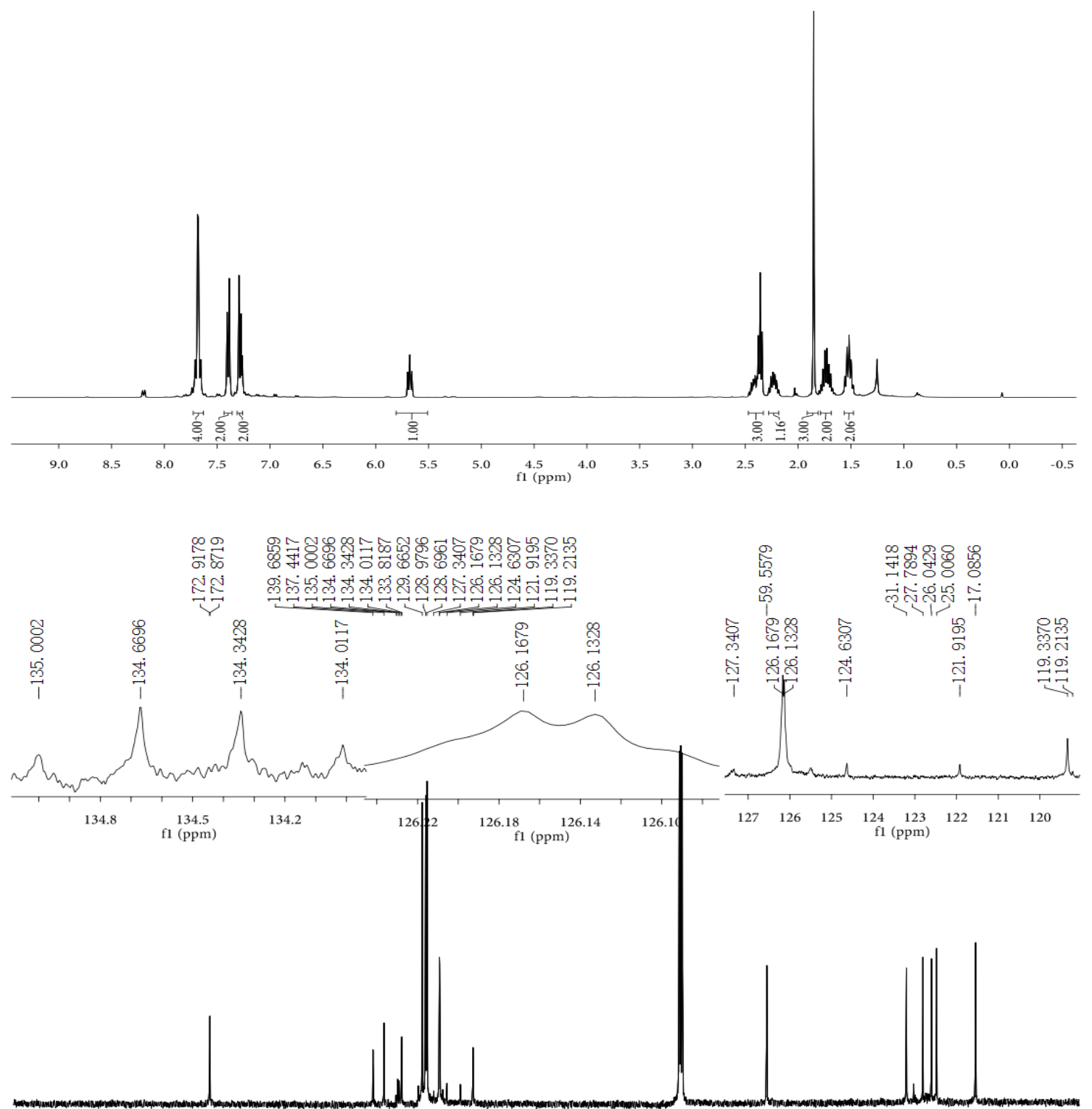


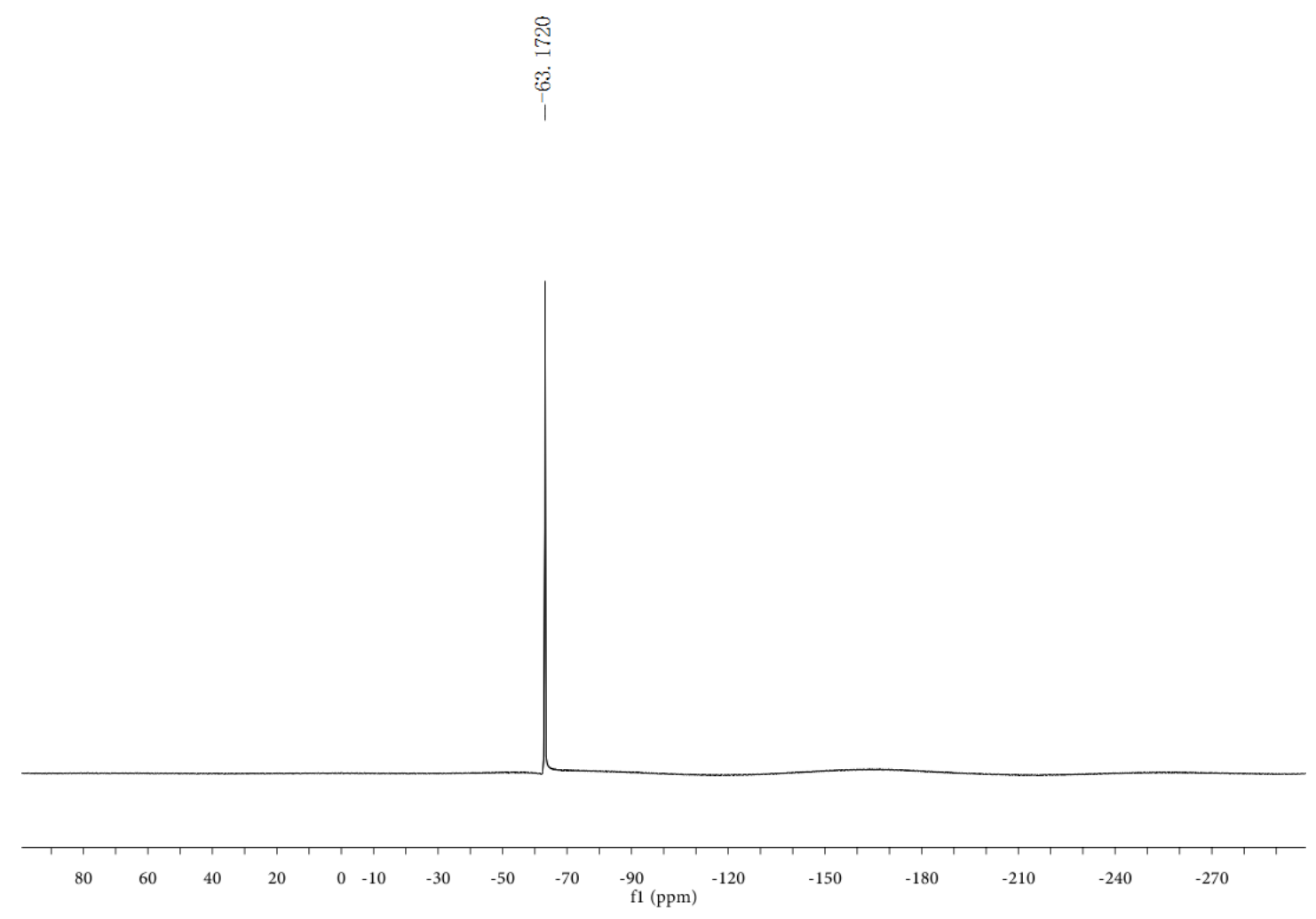


$N$-acetyl- $N$-(1-(4-bromophenyl)-5-cyanopentyl)-4-(trifluoromethyl)benzamide (55):<smiles>CCCCCC(c1ccc(Br)cc1)N(C(C)=O)C(=O)c1ccc(C(F)(F)F)cc1</smiles>

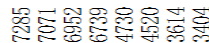

iniving

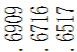

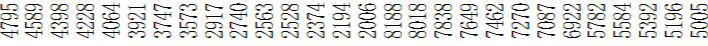

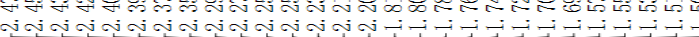

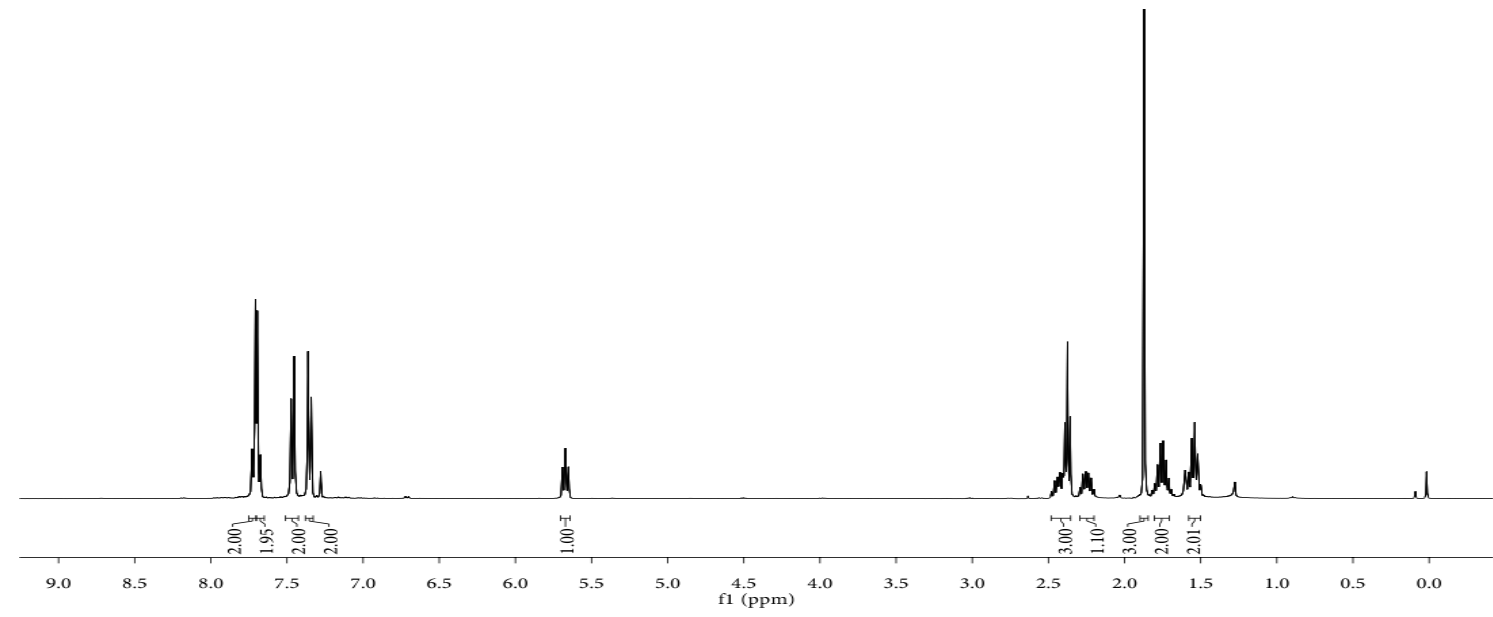

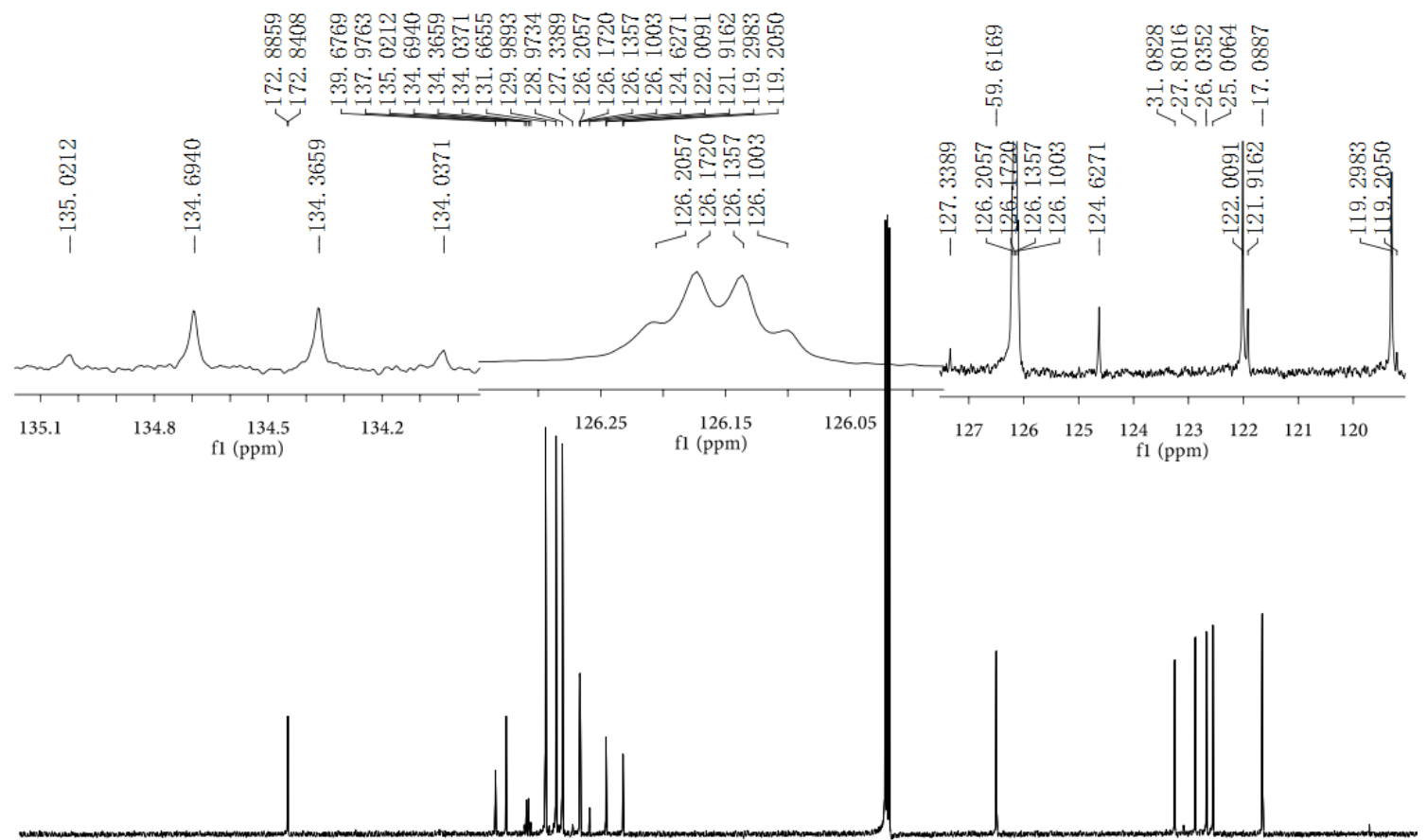

$\begin{array}{rlllllllllll}210 & 200 & 190 & 180 & 170 & 160 & 150 & 140 & 130 & 120 & 110 & 100 \\ \mathrm{f} 1(\mathrm{ppm})\end{array}$ 


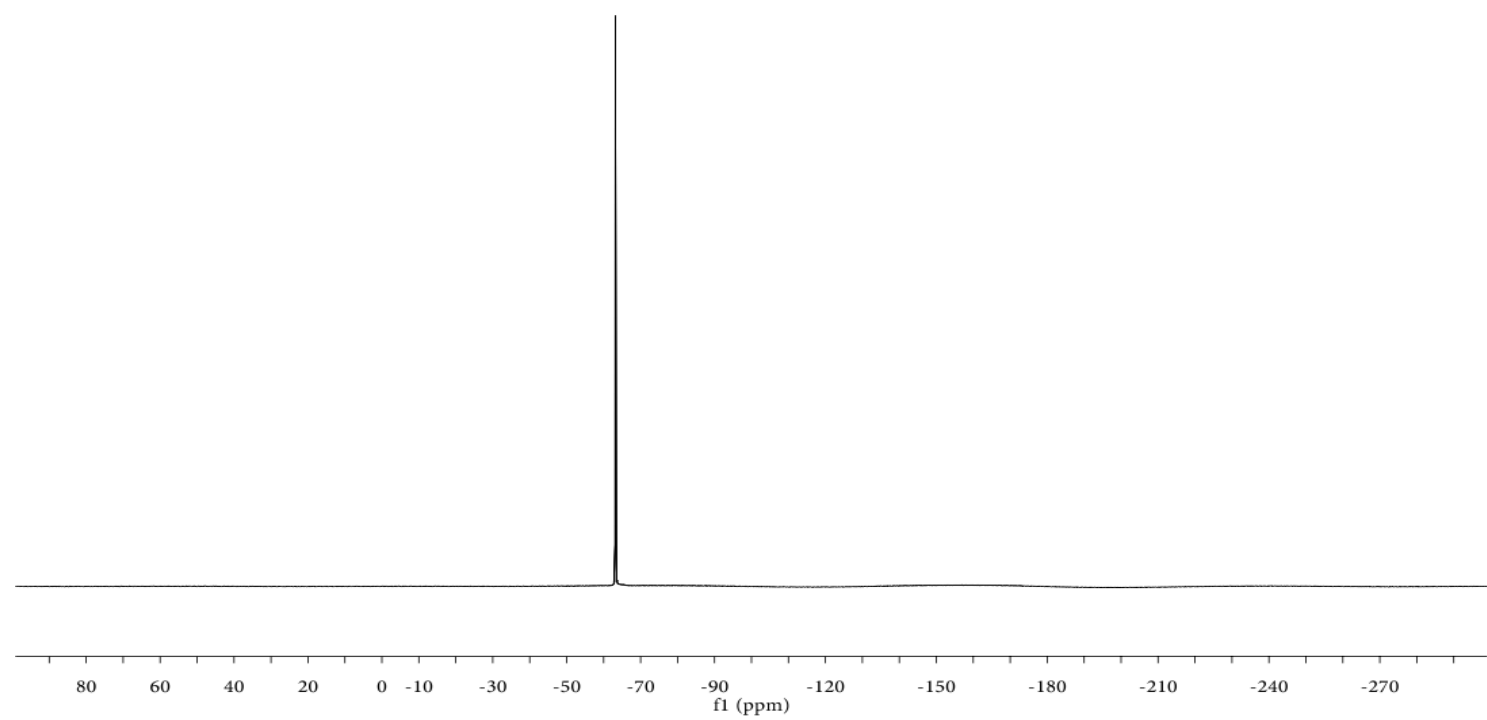


$N$-(1-([1,1'-biphenyl]-4-yl)-5-cyanopentyl)- $N$-acetyl-4-(trifluoromethyl)benzamide (56):

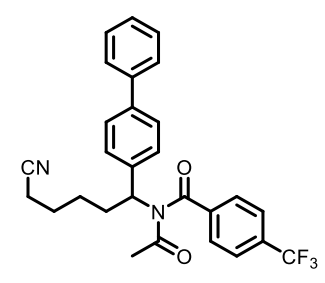

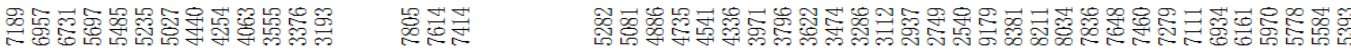

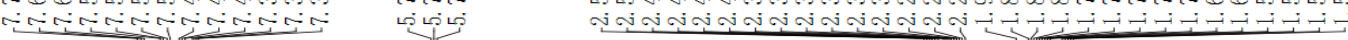
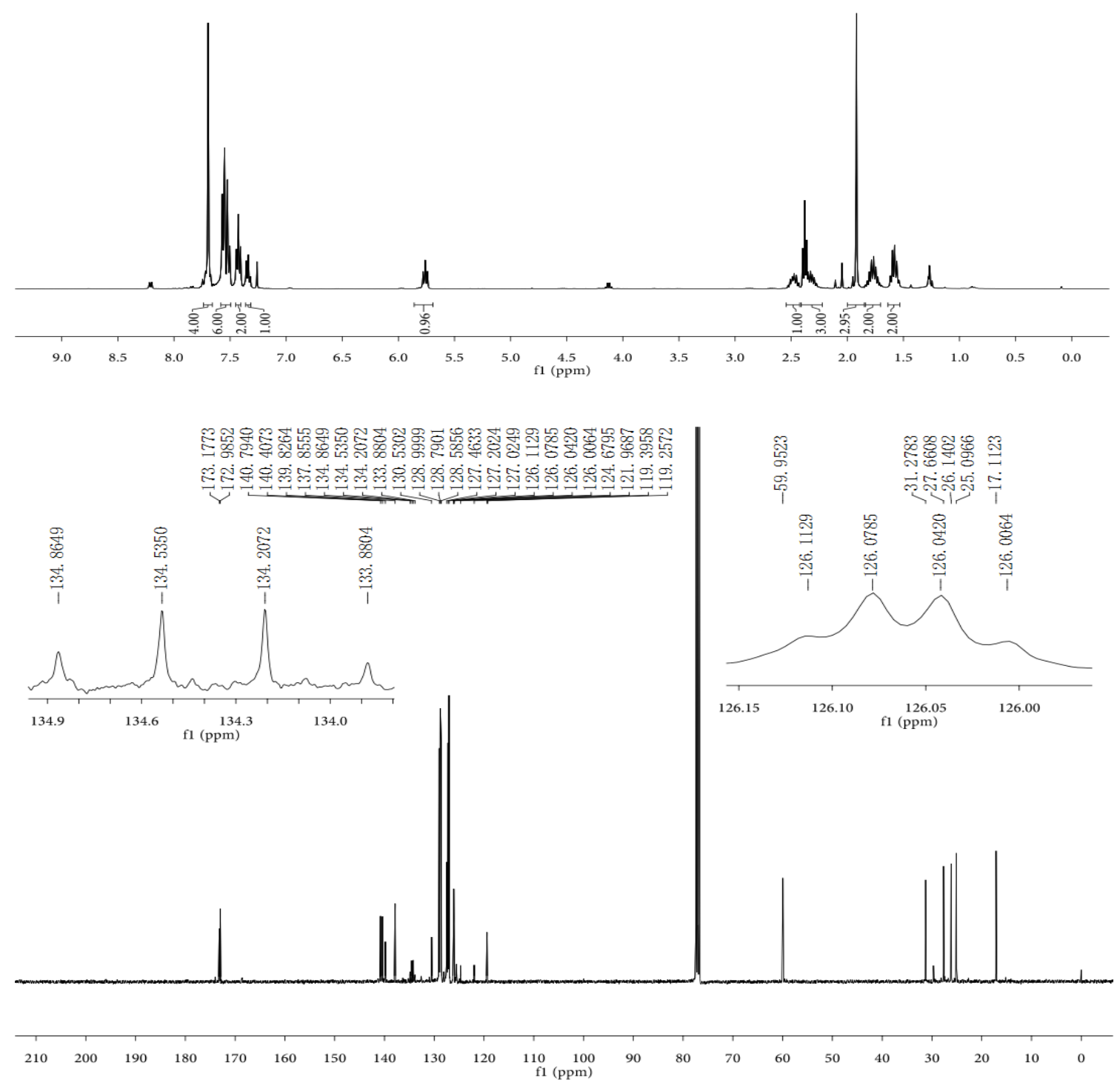


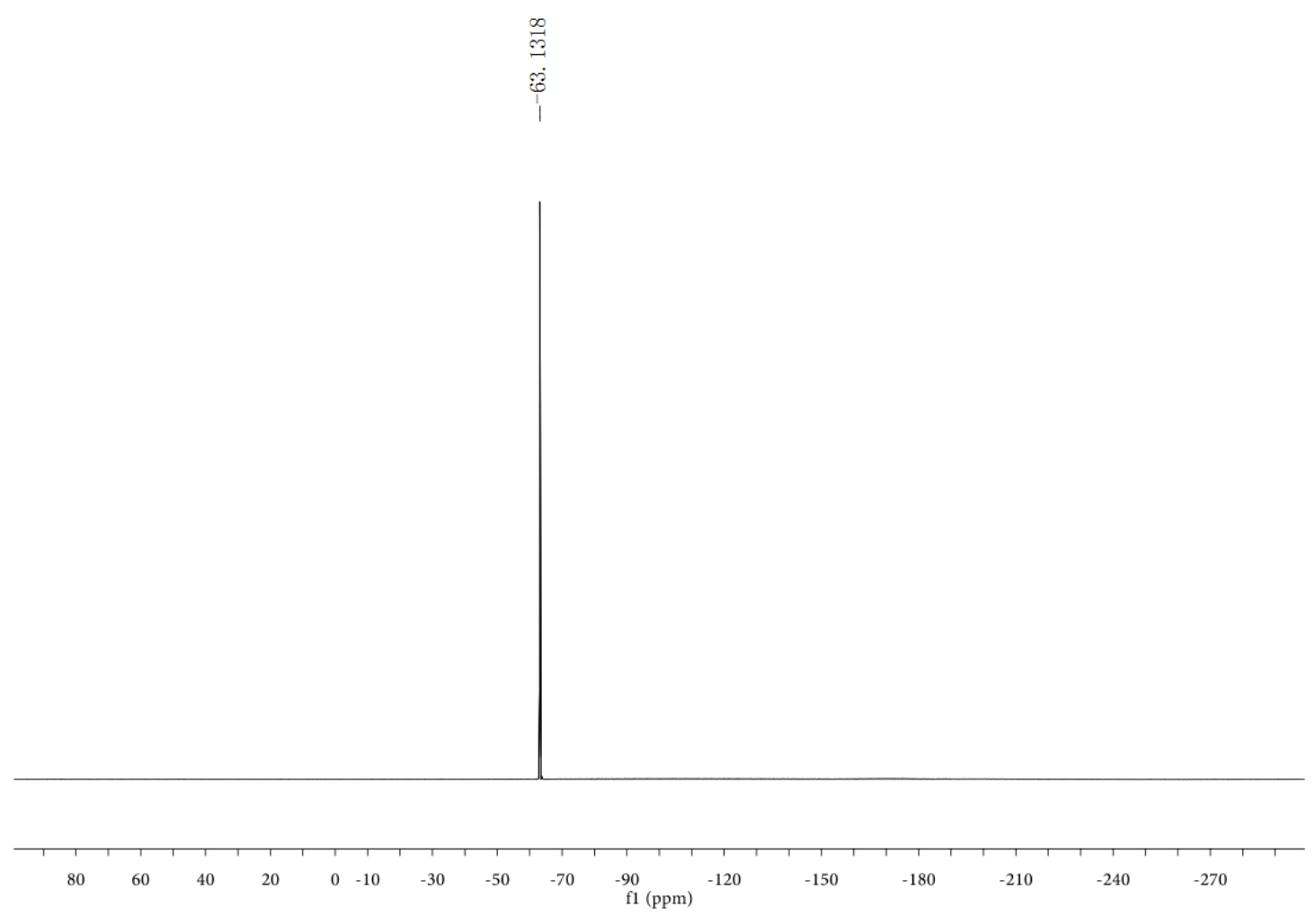


2,2,6,6-Tetramethylpiperidin-1-yl benzoate (57):<smiles>CC1(C)CCC(C)(C)N1C(=O)c1ccccc1</smiles>

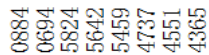

onoinititis

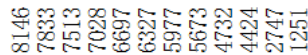

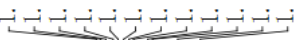

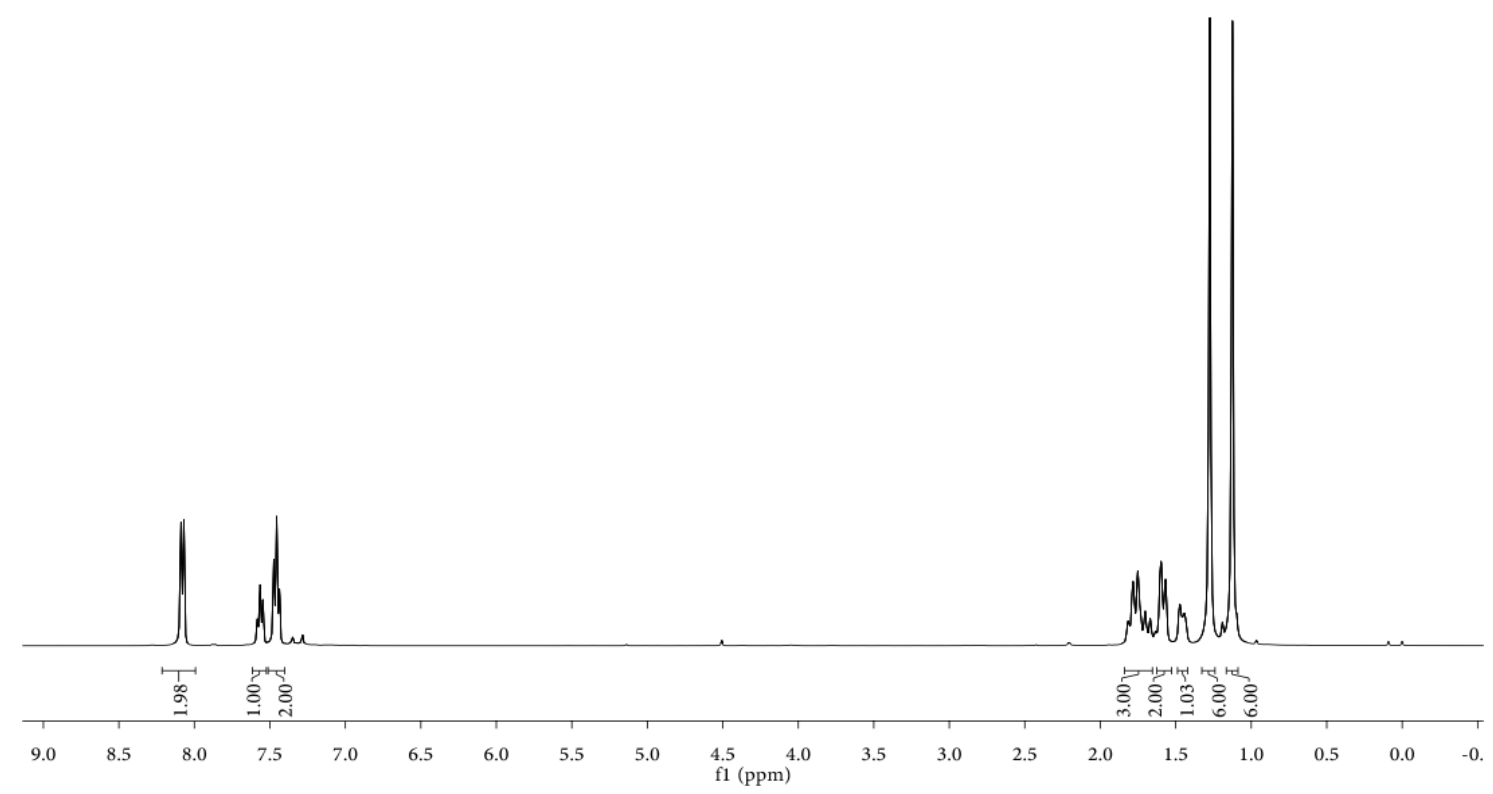

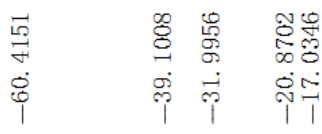

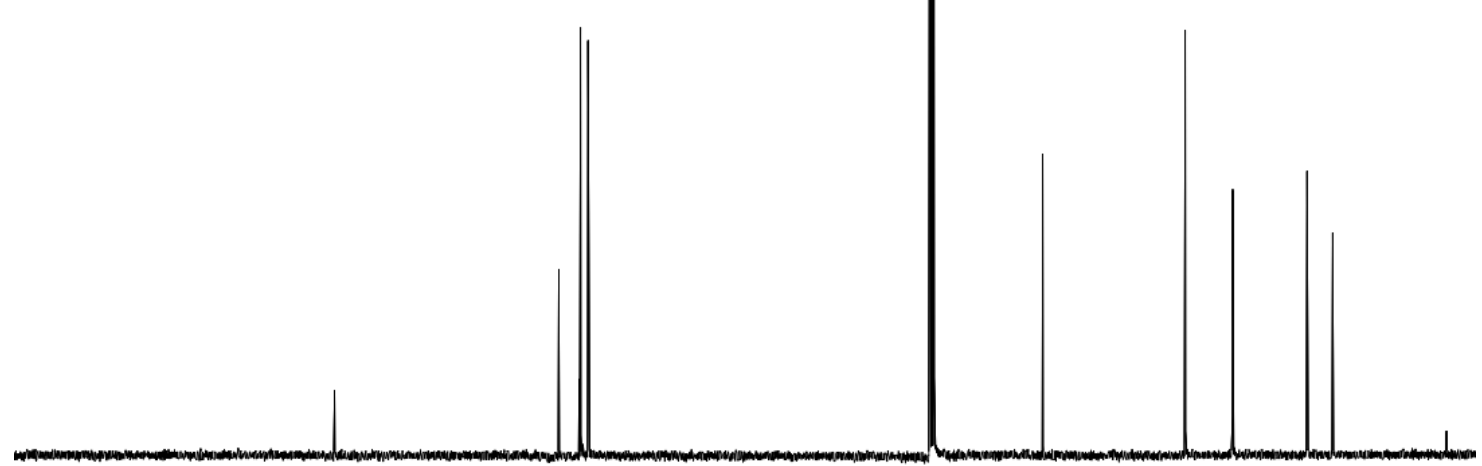

$\begin{array}{lllllllllll}210 & 200 & 190 & 180 & 170 & 160 & 150 & 140 & 130 & 120 & \begin{array}{l}110 \\ \mathrm{f} 1(\mathrm{ppm})\end{array}\end{array}$ 
3-Ethoxy-1,3-diphenylpropan-1-one(59):<smiles>O=C(c1ccccc1)C1CCCC1</smiles>

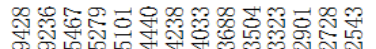

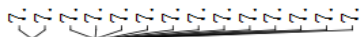

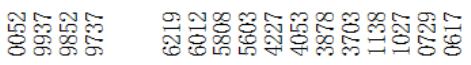

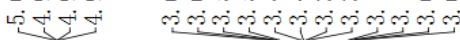

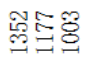

iं山i
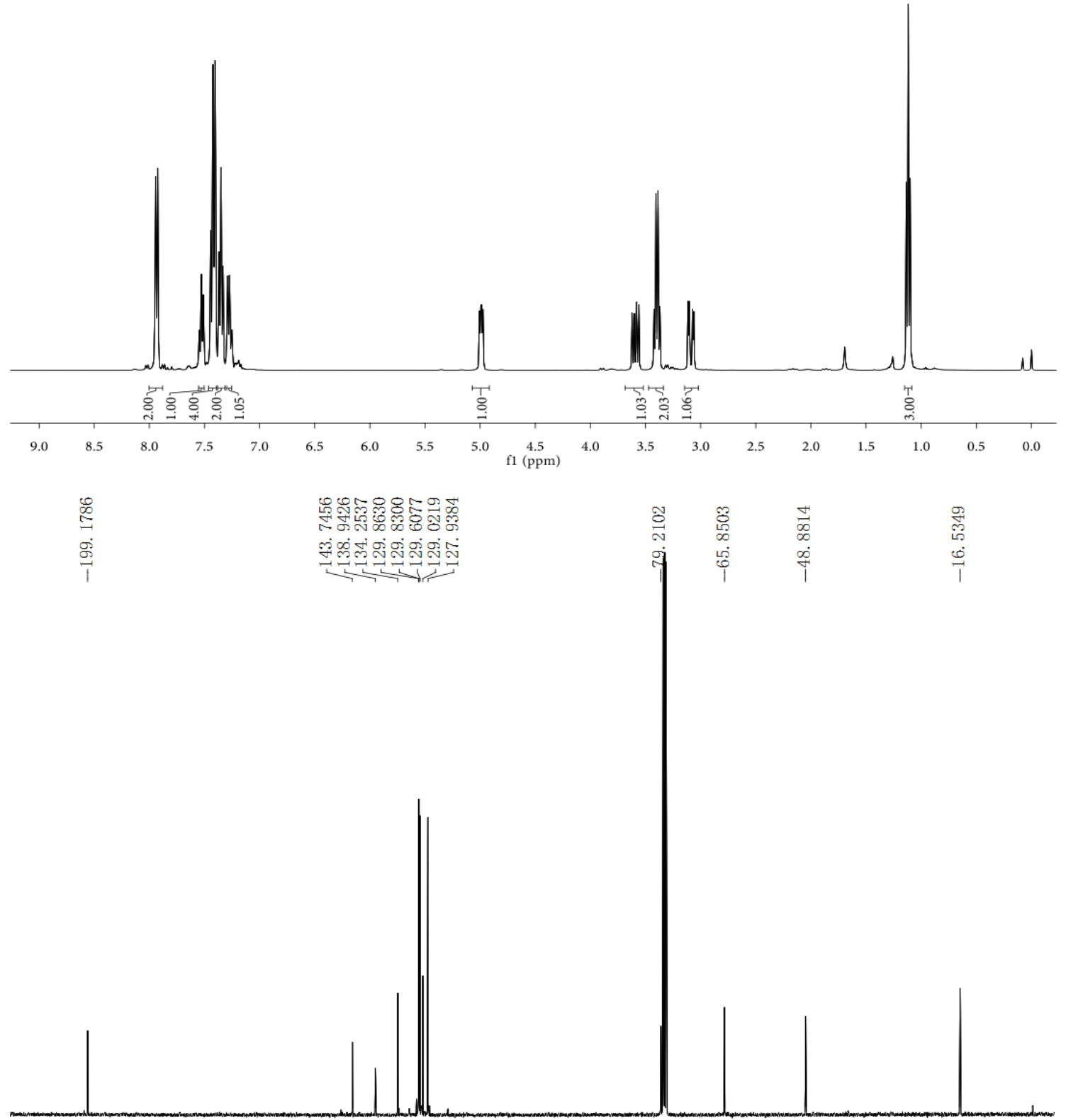

$\begin{array}{lllllllllllllllllllll}210 & 200 & 190 & 180 & 170 & 160 & 150 & 140 & 130 & 120 & \begin{array}{l}110 \\ \mathrm{f} 1(\mathrm{ppm})\end{array} & 90 & 80 & 70 & 60 & 50 & 40 & 30 & 20 & 10 & 0\end{array}$ 


\section{References}

(1) Mohammed, A. H. A.; Nagendrappa, G., A Remarkably Simple alpha-Oximation of Ketones to 1,2-Dione Monooximes Using the Chlorotrimethylsilane-isoamyl Nitrite Combination. Tetrahedron Letters 2003, 44, 2753-2755.

(2) Fan, X.; Lei, T.; Chen, B.; Tung, C. H.; Wu, L. Z., Photocatalytic C-C Bond Activation of Oxime Ester for Acyl Radical Generation and Application. Org. Lett. 2019, 21, 4153-4158.

(3) Shen, X.; Zhao, J. J.; Yu, S. Y., Photoredox-Catalyzed Intermolecular Remote C-H and C-C Vinylation via Iminyl Radicals. Org. Lett. 2018, 20, 5523-5527.

(4) Yu, X.-Y.; Chen, J.-R.; Wang, P.-Z.; Yang, M.-N.; Liang, D.; Xiao, W.-J., A Visible-LightDriven Iminyl Radical-Mediated C-C Single Bond Cleavage/Radical Addition Cascade of Oxime Esters. Angew. Chem. Int. Ed. 2018, 57, 738-743.

(5) Yu, X. Y.; Zhao, Q. Q.; Chen, J.; Chen, J. R.; Xiao, W. J., Copper-Catalyzed Radical CrossCoupling of Redox-Active Oxime Esters, Styrenes, and Boronic Acids. Angew. Chem. Int. Ed. 2018, 57, 15505-15509. 